\title{
Logical Studies in Early Analytic Philosophy
}

NINO B. COCCHIARELLA 
Copyright (1) 1987 by the Ohio State University Press.

All rights reserved.

Library of Congress Cataloging-in-Publication Data

Cocchiarella, Nino B.

Logical studies in early analytic philosophy.

Bibliography: p.

Includes index.

1. Logic, Modern-20th century. 2. Analysis

(Philosophy)-History. I. Title.

BC38.C62 $1987 \quad 160^{\prime} .9^{\prime} 041 \quad 87-1584$

ISBN 0-8142-0437-6 
Dedicated to my grandsons,

Nino Pasquale and Steven James Cocchiarella 


\section{Contents}

Preface $x i$

Introduction 1

1. On the Origin and So-Called Demise of Analytic Philosophy 1

2. The Development of the Theory of Logical Types in Early Analytic Philosophy 5

3. Frege, Russell, and Logicism 8

4. Russell, Meinong, and the Logic of Nonexistence 11

5. Russell, Wittgenstein, and Logical Atomism 12

1 The Development of the Theory of Logical Types and the Notion of a Logical Subject in Russell's Early Philosophy 19

1. Logical Subjects and the Univocity of Being in the Principles of Mathematics 20

2. The Class as Many versus the Class as One 21

3. The Class as Many as a Plural Logical Subject 23

4. Propositional Functions as Non-Entities 25

5. Propositions as Single Logical Subjects 29 
6. The Substitutional Theory of Classes 33

7. Propositions versus Statements in the Substitutional Theory 38

8. The 1908 Theory of Logical Types 43

9. Russell's 1910 Multiple Relations Theory of Judgment 48

10. The Theory of Ramified Logical Types 52

11. Concluding Remarks on Russell's 1925 Introduction to Principia Mathematica 56

2 Frege, Russell, and Logicism: A Logical Reconstruction 64

1. Logicism and the Predicative Nature of Concepts 65

2. Predication versus Functionality 70

3. Existential Posits and the Laws of Logic 72

4. Wertverläufe as Concept-Correlates 76

5. Frege's Double Correlation Thesis and His Basic Law V 79

6. Russell and Frege on Nominalized Predicates 80

7. Russell's Paradox Revisited 83

8. Frege's Rejection of Schröder's Hierarchy of Individuals 89

9. Frege's Double Correlation Thesis and the Theory of Simple Logical Types 92

10. The Theory of Homogeneous Simple Types as a Second Order Logic 94

11. Frege and the Principle of Extensionality 97

12. Russell and the Principle of Rigidity 99

13. Frege's Contemplation of the Abelardian Vicw 102

14. A Second Reconstruction of Frege's Logicism 105

15. An Intensionalized Form of Frege's Logicism 108

16. Russell's Logicism as Conceptual Platonism 111

3 Meinong Reconstructed versus Early Russell Reconstructed 119

1. Meinongian Objects versus Russellian Individuals 119 
2. Russellian versus Meinongian Definite Descriptions 122

3. An Orthodox "Picture" of Meinongian Objects 129

4. A Russellian "Picture" of the Nuclear/Extra-Nuclear Distinction 133

5. Why Parsons's Plugging up of Relations is Unnecessary 141

6. Properties and Relations as Individuals 143

7. Will the Real Fictional Objects Please Stand Up 147

4 Frege's Double Correlation Thesis and Quine's Set Theories NF and ML 152

1. NF and the Iterative Concept of Set 153

2. The Theory of Simple Types and Frege's Double Correlation Thesis 157

3. Frege's Logicism as a Second Order Predicate Logic with Nominalized Predicates 160

4. The Theory of Homogeneous Simple Types as a Second Order Predicate Logic 165

5. Quine's Thesis and the Similarity of NF with $\lambda \mathrm{HST}^{*}$ $+\left(\mathrm{Ext}^{*}\right)+\left(\mathrm{Q}^{*}\right) 169$

6. On Taking Urelements Seriously 172

7. An Alternative Modification of Frege's Double Correlation Thesis 176

8. Ultimate Classes and the Similarity of ML with HST: $+\left(\mathrm{Ext}^{*}\right)+\left(\mathrm{Q}^{*}\right) 181$

9. On Mathematical Induction and the Class of Fregean Natural Numbers 186

5 Russell's Theory of Logical Types and the Atomistic Hierarchy of Sentences 193

1. The 1910 versus the 1908 Theory of Logical Types 196

2. Propositional Functions as Properties and Relations in Russell's 1910-13 Principia MathematiciaOntology 200 
3. Russell's 1910-13 Commitment to Abstract Facts 203

4. Logical Atomism and the Doctrine of Logical Types 206

5. Propositional Functions as Linguistic Conveniences 211

6. Russell's Weakened Form of the Principle of Atomicity 217

6 Logical Atomism and Modal Logic 222

1. Atomic Situations and Complementation 224

2. Elementary Propositions and Complementation 226

3. Propositional Connectives as Punctuation Marks 227

4. Semantic Ascent/Or Was This Trip Really Necessary? 228

5. Zeigen versus Sagen 231

6. The Propositional Modal Calculus S13 238

7 Logical Atomism, Nominalism, and Modal Logic 244

1. Negative Facts and Complementary Nexuses 247

2. Nominalism's View of Logical Space 252

3. An Abstract Semantics for Nominalist Logical Atomism 259

4. The (Onto)logical Grammar of Nominalist Logical Atomism 262

5. The Problem of Identity in Logical Atomism 267

6. Logical Truth versus Logical Necessity 272

7. The Incompleteness of Nominalist Logical Atomism 274 


\section{Preface}

The essays collected here deal with the development of analytic philosophy in the first quarter of the twentieth century. In addition to providing a historical account of early analytic philosophy, these essays also contain logical reconstructions of Frege's, Russell's, Meinong's, and Wittgenstein's views during the period in question. Several of these reconstructions can and have been used in the new logicolinguistic developments in pragmatics and intensional logic that make up the vanguard of contemporary analytic philosophy. Others, such as the interpretation of the logical modalities in logical atomism, or the determination of the objects of fiction and dreams in Meinong's theory of objects or Russell's early logic, provide a useful introduction, if not also a solution, to a number of problems confronting analytic philosophy today. Indeed, for that matter, all of the essays collected here provide a useful propaedeutic to much of the research now going on in the study of logic and language.

A number of small changes have been made in all of the essays reprinted here, mainly for stylistic purposes. Their histories are bricfly indicated as follows. Chapter 1 first appeared in Synthesé, vol. 45, no. 1 (September 1980):71-115, Copyright (c) 1980 by D. Reidel Publishing Company, Dordrecht, Holland. A somewhat longer version of chapter 2 first appeared in Frege Synthesized, L. Haaparanta and J. Hintikka (eds.), 1986, pp. 197-252, Copyright (c) 1986 by D. Reidel Publishing Com- 
pany, Dordrecht, Holland. The present version was given as a lecture in a seminar on 13 March 1985, for the Bertrand Russell Editorial Project at McMaster University. Chapters 3 and 4 first appeared in Journal of Philosophical Logic, vol. 11, no. 2 (May, 1982): 183-214, and vol. 14, no. 1 (February 1985):1-39, respectively, Copyright (C) 1982 by D. Rcidel Publishing Company, Dordrecht, Holland. Chapter 3 was originally given as a lecture to the Sociéte Belge de Logique et de Philosophie des Sciences, Brussels, in December 1981. Chapter 4 was my contribution to An Interdisciplinary Conference on Logic, Truth and Type Theory, given in memory of Alfred Tarski, 6-7 April 1984. Chapter 5 first appeared in Essays in Bertrand Russell's Philosophy, C. Wade Savage and C. Anthony Anderson (eds.), 1987, Copyright (C) by University of Minnesota Press, Minneapolis. Chapter 6 first appeared in Philosophia, Pbilosophical Quarterly of Israel, vol. 4, no. 1 (January 1974):41-66. It is reprinted here with the permission of the editor. Chapter 6 was given as a lecture to the Victoria Conference on Formal Ontology at the University of Victoria on 15 October 1972. Chapter 7 first appeared in Synthesé, vol. 31, no. 1 (June 1975):23-62, Copyright (C) 1975 by D. Reidel Publishing Company, Dordrecht, Holland. It was originally given as a lecture to the University of North Carolina Fall Philosophy Colloquium in October 1973. 


\section{Logical Studies in}

Early Analytic Philosophy 


\section{Introduction}

\section{On the Origin and So-Called Demise of Analytic Philosophy}

Analytic philosophy is sometimes said to have begun with the metaphysics of logical atomism and to have ended with the antimetaphysics of logical positivism, where the end was brought on in part, if not entirely caused by, British ordinary language philosophy. ${ }^{1}$ Nothing could be further from the truth, however, both in regard to the origin of analytic philosophy and its so-called demise.

Logical atomism is indeed part of early analytic philosophy; but it is really only the second part of a two-fold metaphysical development that was subsequently replaced by the antimetaphysics of logical positivism. The first part of that development, which is really the initial period in the history of analytic philosophy, began at the turn of the century in the revolt against absolute idealism. The leaders of this revolt were Bertrand Russell and G. E. Moore, both of whom initially advocated what some have called an "extreme" form of metaphysical realism. ${ }^{2}$ Along with this realism, at least in Russell's case, there was the notion of a logically perfect language as a regulating ideal relative to which all philosophical analyses were ultimately to be given. It is this notion that is fundamental in analytic philosophy, and, in particular, it is the construction and methodology of applying such a regulating ideal as a theory of logical form that is the goal of analytic philosophy. It is this notion, moreover, that is a 


\section{Introduction}

basic theme in logical atomism, where the metaphysics, albeit still realist, is far more parsimonious. Given the rejection of the earlier so-called "extreme" form of realism and the parsimony of logical atomism's, it is no wonder that logical atomism is sometimes wrongly taken to be the origin of analytic philosophy.

Now the notion of a theory of logical form as a regulating ideal relative to which all philosophical analyses are ultimately to be given can be realized in different ways depending on what metaphysical, or antimetaphysical, assumptions are taken as the basis for its development. In particular, as is explained in chapter 5 , the form that that ideal took in logical atomism turns out to be a radically revised and restricted version of the form that preceded it in the initial period of analytic philosophy; and this is so precisely because logical atomism is based on metaphysical assumptions that are different from those of the earlier period. Thus whereas the metaphysical framework of the initial period can be described as one or another version of logical realism, the metaphysical framework of logical atomism is really a version of natural realism. ${ }^{3}$ How the latter developed out of the former, and how they determine different theories of logical forms, are questions we take up in the following essays. Both, despite their differences, belong together as part of the metaphysical phase of early analytic philosophy, and, for this as well as other reasons, both are to be distinguished from the later antimetaphysical period of logical positivism.

Analytic philosophy did not begin, accordingly, with logical atomism. Neither, as we shall see, did it really end with logical positivism, the principal features of which are (1) the verificationist theory of meaning, (2) the rejection of all metaphysical statements as meaningless, and (3) the notion of a logically correct language in which (1) and (2) might be realized. Thus, although the construction and methodology of a theory of logical form as a regulating ideal is a goal logical positivism shared with the earlier periods of analytic philosophy, in logical positivism it is not a goal based on metaphysical but on antimetaphysical assumptions. In particular, on these assumptions, metaphysical statements only appear to be meaningful in ordinary or natural language, whereas in fact they are really meaningless; and that they are meaningless was something that could be shown by their not having any analysis or translation into a logically correct language that is semantically in accord with the verificationist theory of meaning. 
Now it is noteworthy that the notion of a logically correct language in which all metaphysical statements would be rejected as meaningless was part of a visionary goal that logical positivism actually adopted from logical atomism. The oddity, if not outright incoherence, that logical atomism should proclaim such a goal while being itself a metaphysical system is what in part led to its rejection by logical positivism. There is no such oddity in the first or initial period of analytic philosophy, however, where metaphysical statements are in general taken as meaningful, though not in all, cases also as true. In this regard we should distinguish the notion of a logically perfect language or theory of logical form in which metaphysical theses can be embodied, regardless of whether or not they are also assumed to be true, from the notion of a logically correct language in which all metaphysical statements are to be rejected as meaningless. The first is a completely coherent notion that was an integral part of analytic philosophy in its initial period; and the fact that it was originally defended in the context of an "extreme" form of realism in no way precludes either its coherence or its utility for other metaphysical contexts as well. The notion of a logically correct language in which all metaphysical statements are rejected as meaningless, on the other hand, is essentially self-refuting, since such an ideal language will itself embody a metaphysical system either outright, as in logical atomism, or indirectly, as in logical positivism where the verificationist theory of meaning, not being itself verifiable even in principle, must then be taken as a metaphysical thesis. It is only the first notion of a logically perfect language, or theory of logical form as a regulating ideal, that is our concern in the essays collected here.

The visionary, and ultimately self-refuting, goal that logical positivism set for itself was subsequently attacked and rejected by what later came to be called ordinary language philosophy. No distinction was made in that rejection, however, between (1) the positivists' notion of a logically correct language in which a good deal of ordinary language would be rejected as meaningless, and (2) the notion of a logically perfect language in the sense of a theory of logical form that could serve as a regulating ideal by which to explain even the semantics of ordinary or natural language. This was because ordinary language philosophy, although it does not reject analysis as a philosophical method, assumes that no formal language or theory of logical form can be used for that purpose. Philosophical analyses, in other words, are to be restricted to the actual use of ordinary 
language, that is, to the different ways that expressions of natural language function in different possible contexts of use; and, in addition, no system of logical syntax or theory of logical form was to be taken as the semantical basis of any such analysis. This is a rather strong presumption, needless to say, and, as indicated, it goes beyond the claims of logical positivism. Indeed, the assumption simply rejects the basic thesis of analytic philosophy as we understand it here; namely, that the goal of analysis is the construction and methodology of applying a theory of logical form relative to which philosophical analyses can be given, including in particular the analysis of ordinary or natural language. It is no wonder that ordinary language philosophers assumed they had brought about the demise of analytic philosophy.

Now it might be objected that ordinary language philosophy did not so much bring about an end to analytic philosophy as reformulate its goals and methods; that is, that the goal now was to be restricted to the analysis of ordinary or natural language and that the methodology was to eschew any appeal to logical syntax and to be restricted instead to certain paradigm cases of ordinary language usage. We shall not dispute the merits of such a view here, though we do agree that a semantical analysis of the different possible contexts of use of natural language should be of prime importance to analytic philosophy. What is ironic is that such a priority has been taken for granted in what has succeeded ordinary language philosophy where there has been a return to the notion of a theory of logical form as a regulating ideal, and hence a return to the original goal of analytic philosophy. This new form or period of analytic philosophy combines modern linguistic techniques with both recent developments in pragmatics, which takes the notion of a possible context of use as basic, and with intensional logic, which is a theory of logical form that, among other things, plays the role of a regulating ideal regarding validity and logical consequence. There is no attempt on this new approach to reject any part of ordinary language as meaningless; and, in fact, ordinary or natural language has become the testing ground by which to evaluate the adequacy of alternative intensional logics.

One noteworthy feature of these new developments in analytic philosophy is that the intensional logics that have been constructed are similar in many respects to the sort of logic Russell had constructed in the initial pe- 
riod. Ironically, these logics are even based for the most part on essentially the same sort of realist metaphysical framework that Russell had advocated in his early philosophy. That is, both the metaphysical and the logical frameworks of the new and presently ongoing period of analytic philosophy resemble in many respects the metaphysical and logical frameworks of the initial period preceding logical atomism. And this is so, moreover, even though the new practitioners of analytic philosophy have in general failed to recognize or appreciate this similarity. ${ }^{4}$ This is in part the result of an inadequate understanding of Russell's early philosophy, and also in part a confusion, even by Russell himself (as I explain in chapter 5), of the logical framework characterizing the initial period of analytic philosophy with that characterizing logical atomism. One of the purposes of these essays is to clarify our understanding of Russell's early philosophy and to explain the difference between the first and second periods of early analytic philosophy.

Analytic philosophy, accordingly, neither began with logical atomism nor ended with logical positivism; and, what is more important, the metaphysical and logical form it had prior to its development in logical atomism is similar in many respects to the form it presently has in a framework that has surpassed and superseded ordinary language philosophy. It is appropriate, accordingly, that in order to better understand and develop its newest form, we should first have a clear understanding of its initial form and how that form differs from its development in logical atomism.

\section{The Development of the Theory of Logical Types in} Early Analytic Philosophy

During the reign of ordinary language philosophy's "cultural revolution," philosophers committed to formal analyses retreated to applying their skills in definitional extensions of set theory. Set theory, including set-theoretic semantics, became the basic framework in which to develop not only all of mathematics but all of formal philosophy as well. This is a mathematical theory, it should be emphasized, based on the iterative concept of set, and not a theory of logical form based on an analysis of predication and the 


\section{Introduction}

logical notion of a class as the extension of a concept. In this framework, logic is not a language in which to frame all philosophical analyses, including the analysis of mathematics, but only a calculus, one among many, subject to different interpretations over varying domains.

Now with the rise of the new developments in pragmatics and intensional logic there came a renewed interest in type theory both as a theory of logical form and as an alternative framework to set theory. ${ }^{5}$ This was a result, in part, of the fact that type theory, as a theory of logical form, gives a more direct and appropriate analysis of the forms of predication in natural language than does set theory in terms of membership. ${ }^{6}$ In addition, as part of its analysis of predication, type theory also covers the role of nominalized predicates as abstract singular terms. In other words, unlike set theory, which is based on membership and the iterative concept of set, type theory is based on an analysis of predication, including the role of nominalized predicates as abstract singular terms.

The renewed interest in type theory as a framework for intensional logic did not in general bring with it any consideration of its historical ori-

gins or of the kind of issues that led to its development in Russell's early philosophy. This is perhaps understandable in cases (such as Montague Grammar) where Russell's views are not in question and have no particular bearing on the new developments in type theory that are being made. It is not understandable, however, in cases where Russell's views are very much in question, and where what is being claimed is not in conformity with those views. In addition, it is not always clear even in the case of new developments that we are not really rediscovering problems or solutions already covered by Russell. For these reasons I suggest that what is needed as a propaedeutic to the new research in pragmatics and intensional logic is a clear presentation of the kinds of issues that led Russell to develop his theory of logical types in the first place. Chapters 1 and 2 of the following collection of essays were written, in part, with just such a purpose in mind.

Aside from a propaedeutic to the new research in pragmatics and intensional logic, the main purpose of these essays is, quite simply, to explain a development of some importance in the history of philosophy and logic. What in fact were the major issues that led Russell, in his search for a logically perfect language, to the construction of his theory of logical 
types? What, for example, were Russell's initial assumptions regarding the form and content of such a language, and how and why did he modify those assumptions? Is there some fundamental notion or issue, in particular, that provides the key toward understanding Russell's changing views on the ultimate forms of analyses? These are the questions I take up in chapters 1 and 2, and I believe that the answers I provide are important not only for the new developments in pragmatics and intensional logic but for the history of early analytic philosophy as well.

The fundamental notion, for example, that provides the key toward un- $\triangle$ derstanding Russell's changing position, as I explain in chapter 1, is the notion of a logical subject, i.e., the notion of an entity that can be named, mentioned, or denoted, and about which something can be asserted. In the Principles of Mathematics ([POM]), where Russell's "extreme" form of realism (or what in chapter 3 I prefer to call a form of possibilism) was made evident, every entity of whatever sort is a logical subject, or what Russell then also called a term. "Whatever may be an object of thought, or may occur in any true or false proposition, or can be counted as one, I call a term.... I shall use as synonymous with it the words unit, individual, and entity. The first two emphasize the fact that every term is one, while the third is derived from the fact that every term has being, i.e. is in some sense. A man, a moment, a number, a class, a relation, a chimera, or anything else that can be mentioned is sure to be a term; and to deny that such and such a thing is a term must always be false" ([POM]), 43).

Note that among the things that can be logical subjects in $[P O M]$ are properties, relations and propositions, and that all have being in the same sense that concrete particulars do; for "there is only one kind of being, namely being simpliciter" (p. 449). In [POM], in other words, all proper- $2 \mathrm{~s}$ ties, relations and propositions are individuals or terms in the same sense in which concrete particulars are, and in particular all are values of "the true or formal unrestricted variable," i.e., all are values of individual variables. Later, in Principia Mathematica $([P M])$, on the other hand, only particulars are individuals, and though properties and relations are still logical subjects, they are "objects" or values only of variables of a higher order type. That is, in $[P M]$ being is no longer univocal, where everything is a value of the individual variables, but is instead partitioned into an infinity of different logical types. 


\section{Introduction}

\section{Frege, Russell, and Logicism}

Now it should be emphasized that a theory of logical form in which nominalized predicates (and propositional forms) are substituends of individual variables is not the same as a theory of logical types (of third and higher order). In particular, a theory of logical types (of third and higher order) imposes unreasonable constraints on the meaningful use of predicates in natural language that are not imposed in a theory in which nominalized predicates can occur as abstract singular terms on a par with proper names and other singular terms. It is meaningless, for example, in a theory of logical types for a nominalized predicate expression to occur in the subject or argument position of another predicate unless the latter can be assigned a higher type than the former; and therefore it is meaningless in particular for any predicate to occur in a nominalized form in (one of) its in own subject position(s). Thus, the otherwise unproblematic sentence of English, "the property of being a property is a property of itself," is by fiat ruled out as meaningless in a theory of logical types, as is the sentence, "Smith does not think that the property of being philosophically interesting is itself philosophically interesting, even if Jones does." Also ruled out in this way are otherwise unproblematic sentences whose predicates apply to nominal expressions of different types, such as, "Jones thinks that some people are philosophically interesting, as well as that some propositions, properties and relations are too." All of these sentences were taken as meaningful, it should be noted, in the system Russell implicitly had in mind when he first began writing [POM] in 1900, whereas all are ruled out as meaningless in the later framework of $[P M]$.

$\aleph^{\zeta}$ Of course the system Russell originally had in mind in 1900 was subject to the paradox that he discovered in 1901, and it was for this reason that he began revising $[P O M]$ and included the first version of his theory of logical types in Appendix B. This first version, it turns out, as I explain in chapter 2, was the result of Russell's having contacted Gottlob Frege in 1902 about his paradox and how it affected Frege's system as well. The exact relationship between Russell's first theory of types in Appendix B of $[P O M]$ and Frege's hierarchy of concepts of different levels is one of the topics covered in chapter 2 .

Frege was actually Russell's predecessor in the construction of a theory of logical form, and his Begriffsschrift marks one of the truly great ad- 
vances in the history of logic. It is not the system of the Begriffsschrift, however, to which Russell's paradox applies, but the extension of that system that Frege developed in his Grundgesetze der Arithmetik ([Gg]), and which he took to be the logistic framework in which to reduce all of classical mathematics.

The logical system that Frege constructed in $[G g]$ is sometimes $\operatorname{de}^{-} \Delta \Delta^{-}$ scribed as a second order set theory; but, as is explained in chapter 2, that view is quite erroneous. In particular, such a view confuses sets or classes in the mathematical sense of the iterative concept with classes in the logical sense. Thus, whereas on the iterative concept a set has its being in its members, a class in the logical sense is the extension of a concept, and as such, Frege noted, it "has its being in the concept, not in the objects which belong to it" (Posthumous Writings, 183). For Frege, in other words, the logical forms of predication are more fundamental than that of membership, and this is reflected in the fact that the latter is to be analyzed in terms of the former, not conversely as in the set-theoretic view of logic as calculus.

A more appropriate description of Frege's theory, as I explain in chapters 2 and 4, is that it is a second order predicate logic with nominalized predicates taken as abstract singular terms on a par with proper names and other singular terms. That is, the singular terms Frege generates from formulas by application of his smooth breathing abstraction operator are to be understood as the symbolic counterparts of the abstract singular terms or noun phrases generated in natural language by predicate nominalizations. In ordinary language these nominalizations include not only such familiar patterns as 'F-ness', 'F-ity', 'F-hood', and 'being an F', but also infinitives, 'to F', and gerunds, 'F-ing', as well as Frege's own favorite, 'the concept F'. Thus, as I argue in chapter 2, Frege viewed the theory'? of logical forms he developed in $[G g]$ as providing a logical analysis not only of the predicate expressions that occur in natural language, but also of the predicate nominalizations that occur therein as well.

As indicated above, Russell's implicit framework at the turn of the century was also essentially that of a second order predicate logic with nominalized predicates as abstract singular terms, though unlike Frege his account of this framework was presented very informally in [POM] (and in his letters to Frege in 1902 and 1903). This is noteworthy because here, as in Frege's framework, we have the essentials of what constitutes a 
theory of logical form as a regulative ideal relative to which all philosophical analyses are to be given; namely, (1) the basic forms of predication that underlie any possible assertion we might make in language, (2) propositional connectives, (3) quantifiers that reach into predicate as well as subject positions (just as they do in natural language), and (4) nominalized predicates (and propositional forms) as abstract singular

$\Delta$ 'terms. Of course, Russell and Frege did not interpret all of these essentials in the same way, their most important difference, as I explain in chapter 2 , being in what each took nominalized predicates to denote. But these differences are minor compared to the naturalness and power to explain so much by way of analysis of the sort of framework they had in common. That is why Russell's paradox came as such a shock to both.

Russell's attempt to avoid his paradox culminated of course in his theory of logical types, the initial version of which he added to $[P O M]$ as $\triangle \triangle A$ ppendix B. (Appendix $\mathrm{A}$ is a discussion of Frege's logical doctrines.) As is clear from their correspondence, Russell got the idea of his first theory of types from Frege's hierarchy of concepts (and Russell's rejection of Frege's view of the unsaturated nature of concepts). Frege could not himself adopt such a theory, as I explain in chapter 2 , and in fact he did not need to since a solution to Russell's paradox was on hand by a simple modification of what I there call his double correlation thesis (regarding the replacement of higher-level concepts by lower level concepts, and ultimately by the objects or individuals of lowest level). Indeed, there are in fact two ways to modify Frege's double correlation thesis, it turns out, and both lead to a logical reconstruction of Frege's original framework. Both, moreover, can be shown to be consistent (relative to weak Zermelo set theory), and, as I explain in chapter 4, both have interesting connections with Quine's two set theories $\mathrm{NF}$ and ML. In addition, the first can be intensionalized and reinterpreted as a logical reconstruction of Russell's own implicit framework in 1904 without the intrusion of a theory of logical types as a grammatical condition for the well-formedness of formulas.

In other words, besides giving an historical and conceptual analysis of the logical situation Russell and Frege found themselves in at the turn of the century, these essays also contain two logical reconstructions of Frege's original system, one of which can be developed into a reconstruction of the system that Russell implicitly wanted to maintain by the end of his correspondence with Frege in 1904. Both reconstructions return us to 
the very natural framework of a second order predicate logic with nominalized predicates briefly indicated above, a framework that can be used to great advantage in the new developments now going on in pragmatics and intensional logic. ${ }^{7}$

\section{Russell, Meinong, and the Logic of Nonexistence}

There is a trend in some of the new work in analytic philosophy to return not to Frege's or Russell's early frameworks but to Alexius Meinong's instead; that is, to reconstruct Meinong's theory of objects instead of, for example, Russell's early form of realism. The reason, apparently, is that Meinong's theory was designed to account not only for existent or even merely possible objects but for impossible objects as well, such as the objects we might refer to in fiction or dreams. An adequate account of the semantics of ordinary or natural language, in other words, must include an account of all of the objects we purport to refer to, whether in fiction, dreams or reality, and whether existent, possible, or impossible. One of the best and clearest reconstructions of Meinong's theory can be found in Terence Parsons's Nonexistent Objects. I compare this reconstruction with my own reconstruction of Russell's early form of realism (which is really a form of possibilism) in chapter 3. My main thesis there is that Meinongian objects, and impossible objects in particular, are parasitic upon Russellian individuals, and that we do not need to introduce impossible individuals into our reconstruction of Russell's early framework in order to account for the objects of fiction or dreams.

Now it is not generally recognized that Russell was committed to a form of possibilism prior to his 1905 theory of denoting. Indeed, Russell's 1905 paper, "On Denoting," is now so well-known and widely read that his explicit avowal of actualism in that paper-an avowal that he maintained for the rest of his philosophical career-is frequently taken to be the position he held even in $[P O M]$. As some of our earlier quotes clearly indicate, however, this in fact is not the case; that is, Russell was definitely committed in $[P O M]$ to the idea that some individuals that could have existed, had the world been otherwise than the way it is, in

fact do not exist. Russell, in other words, was committed in $[P O M]$ to there being possible individuals that do not in fact exist, i.e., individuals that would have existed had certain propositions (of which they are con- 
stituents) been true that are in fact false. Some of the details of the form of possibilism implicit in Russell's early framework are described in chapter 3.

There is a different mistake that is sometimes made by those who do realize that Russell was committed to nonexistent individuals prior to his 1905 theory of denoting; namely, the mistake of thinking that Russell agreed with Meinong and assumed in his pre-1905 theory of denoting that every definite description denoted, and in particular that it denoted an object that fulfilled the conditions of the description. ${ }^{8}$ That would mean that in his "extreme" form of realism Russell allowed not only for nonexistent possible individuals but impossible individuals as well; that is, that Russell's early framework was really a variant of Meinong's rather than a form of possibilism.

$\triangleleft^{r}$ In fact, however, the theory of denoting in [POM] is explicitly designed to allow for denoting expressions that denote nothing; and, as I explain in chapter 3, Russell even gave a "complete definition of a denoting concept which does not denote anything" ([POM], 74). In particular, on that definition, no definite description that contains contradictory conditions could ever denote anything. Thus, although Russell assumed in his early framework that "everything is real that common sense, uninfluenced by philosophy or theology, supposes real," nevertheless, in taking imaginable things to be individuals or logical subjects, Russell was clear in maintaining only that "everything imaginable, may, if not self-contradictory, ¿be a subject." 10

Finally, it should also be noted that although Russell explicitly excluded merely possible individuals from his framework once his 1905 theory of denoting was formulated, it nevertheless should not be thought that the 1905 theory is in any way incompatible with Russell's early form of possibilism. Nor, on the other hand, should it be thought that Russell's earlier theory of denoting in $[P O M]$ in some way requires or implies possibilism. Either theory of denoting, in other words, can be incorporated in a logical reconstruction of Russell's early framework. ${ }^{11}$

\section{Russell, Wittgenstein, and Logical Atomism}

Although analytic philosophy did not begin with logical atomism, logical atomism is nevertheless part of early analytic philosophy. This is because 
it is part of the metaphysical rather than the antimetaphysical period of analytic philosophy, a period we have returned to in the new developments in intensional logic, and also because it is the outcome, under Wittgenstein's and Frege's influence, of Russell's rather involuted views on the notion of a logical subject. What is somewhat surprising about this outcome is that Russell did not himself realize the effect it had on his theory of logical types, and that in fact all that was left of that theory as a result of his new view of what could be a logical subject was ramified second order logic. This consequence and how Russell came, however unwittingly, to be committed to it is the subject of chapter 5 .

Logical atomism, like the initial period of analytic philosophy, is based on a form of metaphysical realism; but unlike the initial period, the realism is a form of natural and not of logical realism. A Russellian way of putting this difference is that in logical atomism there are only simple properties and relations, namely the material properties and relations of the atomic facts that make up the world, whereas in logical realism there are complex properties and relations as well; and in the logical realism of the initial period there are also (higher order) properties and relations of properties and relations, as well as properties and relations of particulars (or objects of lowest order). Of course, not all forms of logical realism need be based on the complex versus simple distinction, in which case the point of the difference is that between realist frameworks in which one or another comprehension principle is taken to be logically valid, as opposed to those in which no such principle, or at least none regarding complex formulas, is to be so taken. ${ }^{12}$ Also, in natural realism every property or relation is realizable in principle, i.e., has instances in some possible world or other, whereas in logical realism there are properties and relations for which it is in principle impossible that they have any instances at all. ${ }^{13}$

The idea that a property or relation is realizable in principle obviously requires some form of modality for its expression. This is thought to be problematic in logical atomism, since a supposedly basic assumption of that framework is the principle of extensionality, a principle that would exclude any form of modality from being expressible therein. Actually, $\triangle$ however, it is not the principle of extensionality that is fundamental to logical atomism but rather the idea that so-called internal properties and relations of objects, situations, or propositions are merely formal and not 
material in content, and that as such they are part of the logical scaffolding of the world and not part of its ontological structure. This means that all real properties and relations, i.e., the material properties and relations that make up the ontological structure of the world, are external properties and relations; or, in other words, that whatever objects have these properties and relations could also not have had them, and that therefore none of these properties and relations are essential to the objects that do have them.

Now the claim I argue and defend in chapters 6 and 7 is that if we are to adequately represent the distinction in logical atomism between logical scaffolding and ontological structure-i.e., the distinction between the merely formal internal "properties" and "relations" that characterize the logical scaffolding of the world and the real external material properties and relations that make up the ontological structure of the world-then we need to introduce modal operators for logical necessity and logical possibility into logical atomism's theory of logical form. In terms of these operators, we are able to make the relevant distinctions and see that what is really fundamental in logical atomism is not the principle of extensionality but rather the modal thesis of antiessentialism instead. For, given these operators, not only does every instance of the modal thesis of antiessentialism become grammatically well-formed, but, in addition, it can easily be shown to be logically true in the abstract semantics described for logical atomism in section 3 of chapter $7 .{ }^{14}$ In addition, as is shown in chapter 6 , given the proper logicogrammatical employment of these modal operators, each possible world of the logical space determined by logical atomism is completely "individuated" by the totality of atomic facts that obtain in that world; that is, the principle of individuation for the possible worlds determined by logical atomism is simply that worlds are "identical" when, and only when, every atomic fact that obtains in the one obtains in the other. Such a result is a complete vindication of the use in logical atomism of modal operators for logical necessity and logical possibility, since it shows in effect that modality is only in the logical scaffolding of the world and not in its ontological structure.

There are other results as well in this modalized version of logical atomism that help us better understand issues now being debated in the new developments of intensional logic. For example, as I explain in chapters 6 and 7 there is nothing in the mere use or introduction of modal op- 
erators for logical necessity and logical possibility that commits one to the existence of possible worlds over and above the atomic facts that might obtain in such a world. In logical atomism in particular, all talk of possible worlds is part of a semantic ladder that in the end can be kicked away. That is not to say, on the other hand, that there cannot be a formal ontology in which it is provable that there are possible worlds in some sense or other. In particular, in the logical reconstruction I give (in section 12 of chapter 2) of Russell's early form of logicism, it is provable that there are possible worlds both in the sense of world propositions (i.e., propositions that are possibly true and that entail, for every proposition $\mathrm{Q}$, either $\mathrm{Q}$ or the complement of Q) and in the sense of maximal "classes" of facts (where "classes," as I explain in chapter 2, are really properties that have the same extension in every possible world). ${ }^{15}$

Another aspect of the semantical analysis I give of logical atomism is also worth noting; namely, the close similarity it brings out between the metaphysics and picture theory of meaning of logical atomism and the set-theoretic model theory of contemporary formal semantics. ${ }^{16}$ Given this similarity, we see why it is important to ask what sort of content is imported into the semantics of the modal operators when their truth clauses are allowed to range over arbitrary sets of models (or worlds of a given logical space), or, similarly, what sort of content is imported into the interpretation of the quantifiers when the domains of these models are allowed to vary (with respect to the same logical space). These questions, as I explain in chapter 7, are relevant to the new developments in intensional logic. ${ }^{17}$

Finally, it should be noted that despite the fact that logical atomism is clearly committed to a form of metaphysical realism, a number of philosophers have attempted to construe it as a form of nominalism. In chapter 7 I examine this attempt in some detail and compare the nominalist construal of logical atomism with two realist versions, one in which material properties and relations are nexuses or modes of configuration of atomic situations, and the other in which material properties and relations are themselves among the objects configured. The difficulties of sustaining a nominalist interpretation are noted throughout chapter 7 , where the point is made that nominalist logical atomism seems to be based on a confusion of the first type of realism with the second, or rather of thinking of only the second type as realist. That is, the attempt to con- $\Delta$ 
strue logical atomism as a form of nominalism is really a confusion of nominalism with the type of realism in which material properties and relations are not objects that can be configured but are instead the nexuses or modes of configurations themselves. This type of realism is in fact the one intended by Wittgenstein in his Tractatus Logico-Philosophicus, and, as is made clear in chapter 5 , it is also the version that Russell adopted after 1913 and his confrontation with Wittgenstein regarding the form of realism he advocated and defended in his original theory of logical types.

Nominalists have perhaps been especially misled by the change in Russell's views after 1913; that is, the shift from the logical realism of his original theory of types to the natural realism of his logical atomism. In particular, it has often been noted that although Russell originally assumed that propositional functions are real universals (or, at least, that a real property or relation corresponded to each propositional function), nevertheless, after 1913, and especially in his philosophy of logical atomism, he changed his mind and explicitly maintained that propositional functions are merely expressions that contain variables and that become sentences when names are put in place of the variables. This shift in the ontological status of propositional functions was misinterpreted by nominalists as a shift not from logical realism to natural realism but from logical realism to nominalism. The proper explanation and significance of this shift is de$\Delta$ 'scribed in some detail in chapter 5 . Nominalism's error in this context is in confusing the semantic significance of the predicates of Russell's atomistic hierarchy of sentences with the purely linguistic significance of the propositional functions that can be constructed on the basis of those predicates. This confusion is not unrelated to the one described in chapter 7 where nominalism fails to distinguish the type of realism in which material properties and relations are the nexuses or modes of configurations of atomic facts (and are not therefore among the objects configured in such facts) from the type of realism in which material properties and relations are themselves objects of such configurations. Both Russell and Wittgenstein rejected the second type of realism in their respective versions of logical atomism, but in doing so, as the following essays make clear, they did not intend to thereby commit themselves to nominalism. Both in fact were maintaining a version of the first type of realism where material properties and relations are the nexuses of atomic facts. 


\section{Notes}

1 Compare J. O. Urmson's Philosophical Analysis (Oxford: Oxford University Press, 1956).

2 Cf. Urmson, Philosophical Analysis, 2.

3 For a discussion of the differences between logical and natural realism, see chapter 3 of my Logical Investigations in Predication Theory and the Problem of Universals (Naples: Bibliopolis Press, 1986).

4 For an illustration of this point and an exposition of the work of one of the most important practitioners of contemporary analytic philosophy, see my paper, "Richard Montague and the Logical Analysis of Language," in Contemporary Philosophy: A New Survey, vol. 2, Philosophy of Language/Philosophical Logic, G. Flostad, ed. (The Hague: Martinus Nijhoff, 1981), 113-55.

5 Cf. Richard Montague's "Pragmatics" (1968), "Pragmatics and Intensional Logic" (1970), and "On the Nature of Certain Philosophical Entities" (1969), all reprinted in Formal Philosophy: Selected Papers of Richard Montague, edited and with an introduction by R. H. Thomason (New Haven: Yale University Press, 1974).

6 Cf. my paper "Predication versus Membership in the Distinction Between Logic as Language and Logic as Calculus," Synthèse (1988).

7 For applications of the first of these reconstructions to linguistics, see Gennaro Chierchia's Topics in the Syntax and Semantics of Infinitives and Gerunds (Ph.D. diss., University of Massachusetts, 1984); and his "Formal Semantics and the Grammar of Predication," Linguistic Inquiry 16 (1985):417-43. For a comparison with Montague's intensional logic, see my "Predication Versus Membership in the Distinction between Logic as Language and Logic as Calculus."

8 Cf. D. Lackey's discussion of Russell's critique of Meinong in Essays in Analysis, D. Lackey, ed. (New York: Braziller, 1973), 19.

9 "My Mental Development," in The Philosophy of Bertrand Russell, P. A. Schillpp, ed. (New York: Tudor Publishing Co., 1944), 12.

10 "An Analysis of Mathematical Reasoning," unpublished manuscript of 1898, p. 10 of Book 1 ; italics added.

11 It should be noted, however, that the $[P O M]$ theory of denoting does conflict with the principle of intensionality, ( $\square$ Ext"), described in section 12 of chapter 2 . This principle is compatible with Russell's 1905 theory, on the other hand, and does seem appropriate to Russell's view of analysis after 1905 (even when adapted to his earlier form of possibilism). Nevertheless, probably the best course is simply to drop the principle of intensionality as part of our logical reconstruction of Russell's carly possibilist framework and allow for a development of either of Russell's theories of denoting.

A logical reconstruction of the $[P O M]$ theory of denoting concepts has a natural application, incidentally, in the conceptual realism framework described in section 16 of chapter 2. A preliminary version of such an application can be found in sections 11-12 of my paper, "Philosophical Perspectives on Quantification in Tense and Modal 


\section{Introduction}

Logic," in Handbook of Philosophical Logic, vol. 2, D. Gabbay and F. Guenthner, eds. (Dordrecht: D. Reidel Publishing Co., 1984), 309-53.

12 Cf. chapter 3 of my Logical Investigations in Predication Theory and the Problem of Universals.

13 Ibid.

14 For more on the logical truth in logical atomism of each instance of the modal thesis of antiessentialism, see section 2 of my paper, "Philosophical Perspectives on Quantification in Tense and Modal Logic."

15 For a fuller discussion and explanation of these two notions of a possible world in my reconstruction of Russell's early form of logicism, see section 5 of my paper, "Predication Versus Membership in the Distinction between Logic as Language and Logic as Calculus."

16 In "Language-Games," Acta Philosophica Fennica, vol. 28, (1976), Jaakko Hintikka commented, "No one has pointed out to my knowledge that there is a remarkable similarity between this [picture] 'theory' [of logical atomism] and the basic ideas of what is known as logical semantics or logical model theory" (p. 105). In fact, I had discussed just that similarity in the preceding year in the essay that is reprinted here as chapter 7.

17 For a fuller discussion of these and related questions, see my paper "Philosophical Perspectives on Quantification in Tense and Modal Logic." 


\section{The Development of the Theory of Logical Types and the Notion of a Logical Subject in Russell's Early Philosophy}

The development of the theory of logical types in Russell's early philosophy proceeds along a difficult and rather involuted path; and even the final product, the theory as adumbrated in $[P M]$, remains unclear in its syntax and problematic in its semantics. Indeed, one might well be left with the impression that Russell himself, in the end, remained unsure of which parts of the different views he had held along the way are finally to be adopted.

In what follows, we shall attempt to describe and explain the development of Russell's early views, at least to the extent to which they are available in published form today, from the perspective of the development in those views of the notion of a logical subject. It is the development of this notion in Russell's early philosophy, we believe, that holds the key to many of the problems confronting Russell in the development of his theory of logical types and that led to the various, and sometimes conflicting, proposals that he made along the way.

It should be noted, however, that in referring to the development of the theory of logical types in Russell's early philosophy we have in mind only the views developed by Russell up to, but not subsequent to, the 1910-13 publication of the first edition of $[P M]$. The subsequent views developed by Russell from 1913-25, that is, between the first and second editions of $[P M]$, and summarized to some extent in his introduction (and added ap- 
pendices) to the second edition, constitute Russell's version of logical atomism. Except for some concluding remarks in the final section of this chapter, we delay our discussion of those views until chapter 5 .

\section{Logical Subjects and the Univocity of Being in the \\ Principles of Mathematics}

It is Russell's view in [POM] that "there is only one kind of being, namely being simpliciter" (p. 449), and it is that being, moreover, that belongs to every logical subject, i.e., "to every conceivable term, to every possible object of thought-in short to everything that can possibly occur in any proposition, true or false, and to all such propositions themselves" (ibid.). Every term, in other words, is a logical subject in the sense that it "may be an object of thought, or may occur in any true or false proposition, or can be counted as one" (ibid., 43). (The word 'term', incidentally, is taken by Russell to be synonymous with 'unit', 'individual' and 'entity' (ibid.); and in this regard, therefore, every entity, according to Russell, is a single logical subject).

Every term, moreover, is a value of the unrestricted variable, i.e., of "the true or formal variable" (ibid., 91), which is to be distinguished from the restricted variable only in the sense that the latter is the unrestricted variable under some hypothesis. Thus every term, according to the Russell of $[P O M]$, is a value of but one type of unrestricted variable, a variable in fact whose logicogrammatical role is the paradigmatic role of a logical subject.

Terms in general, according to this early Russell, are of two fundamental kinds, namely, things and concepts, where "the former are terms indicated by proper names, the latter those indicated by all other words" (ibid., 44). Two particularly important kinds of concepts, moreover, are those indicated by adjectives and those indicated by verbs, also referred to by Russell as predicates and relations in intension, respectively.

The occurrence of a concept in a proposition as a concept, it should be noted, is not the same as its occurrence as a term. Thus, whereas the sentences 'Socrates is human' and 'Humanity belongs to Socrates' express equivalent propositions, according to Russell, the propositions themselves, nevertheless, are not the same. The concept human, in particular, occurs in the first proposition "in a different way from that in which it oc- 
curs when it is called bumanity, the difference being that in the latter case, but not in the former, the proposition is about this notion" (ibid., 45). A concept is a logical subject of a proposition in which it occurs, accordingly, only when the proposition is about that concept, i.e., only when the concept occurs in the proposition as a term.

Propositions, as the first quotation above indicates, are also terms or individuals; and it is noteworthy in this regard that (material) implication is understood in $[P O M]$ to be a relation between terms, albeit one that can obtain only between terms that are propositions. Thus, while it is true that every proposition (materially) implies itself, it is not meaningless but simply false that (say) Socrates (materially) implies himself. It is of course because all and only propositions (materially) imply themselves that Russell is able to define the class-concept proposition as follows (ibid., 15):

$$
p \text { is a proposition }=\mathrm{df} p \text { (materially) implies } p,
$$

where the variable ' $p$ ' that occurs here is but a special case of the unrestricted variable. Russell, to be sure, also uses the variables ' $p$ ', ' $q$ ', etc. as restricted variables having only propositions as their values; but then sentences in which they occur must be implicitly understood as containing a suppressed hypothesis of the form ' $p$ is a proposition', ' $q$ is a proposition', etc.

\section{The Class as Many versus the Class as One}

The fly in the ointment of Russell's univocal sense of being that is the attribute shared by all logical subjects is "the ancient problem of the One and the Many" ( $[P M], 72)$. This is primarily the problem for Russell of the notion of a class as a logical subject, i.e., of a class as one as opposed to a class as many, and secondarily the problem of the notion of a propositional function as a logical subject, i.e., of a propositional function as a single and separate entity as opposed to the propositional function that exists only in the many propositions that are its values.

In regard to the notion of a class, the problem, as Russell restates it in $[P M]$, is that "if there is such an object as a class, it must be in some sense one object, Yet it is only of classes that many can be predicated. Hence, if we admit classes as objects, we must suppose that the same object can be both one and many, which seems impossible" $([P M], 72)$. 


\section{Logical Types and the Notion of a Logical Subject}

Now it is noteworthy in regard to this argument that prior to the discovery of his paradox, Russell "took it as axiomatic that the class as one is to be found wherever there is a class of many" ([POM], 104), whereas after the discovery of his paradox Russell came to believe that it was this assumption that was "the source of the contradiction" (ibid.); and that consequently it was necessary to distinguish a class as many from a class as one. A class as many, as the direction of Russell's original assumption makes clear, is always assumed to exist, and in fact is always taken as an extension of a propositional function. A class as one, on the other hand, might not exist corresponding to a class as many; but if it does, then it is taken to be the whole that is composed of the members of the class as many (cf. [POM], 76, 515).

This distinction between a class as many and a class as one should not be confused, incidentally, with the different contemporary distinction between ultimate or proper classes and classes that are or have sets corresponding to them (as e.g., in von Neumann-Bernays-Gödel set theory). For with respect to the latter distinction, it is in terms of the classes that are or have sets corresponding to them that traditional mathematical sentences are to be modeled, whereas with respect to the former distinction, the modeling, or "analysis," is to be effected in terms of classes as many. It is the class as many, in other words, and not the class as one, that Russell takes to be the range of significance of a propositional function, i.e., the collection of objects for which the function is significant; and it is precisely such ranges that constitute the different logical types in Russell's first attempt at such a theory (in Appendix B of $[P O M]$ ).

Now because it is a whole, the class as one, when it exists, is always a single logical subject, i.e. a term or individual, and in fact it was taken by Russell to be "an object of the same type as its terms" ([POM], 105). ${ }^{1} \mathrm{~A}$ class as many, on the other hand, is not an individual or term, and cannot, therefore, "be treated as a single logical subject" (ibid., 69, italics added). A class as many is not a single entity, in other words, but many; and it was taken as a collection by Russell only in the sense of "a numerical conjunction of terms" (ibid., 73), as is indicated, e.g., by ' $A$ and $B$ ', ' $A$ and $B$ and $C$ ', etc., where it is clear that $A$ and $B$ are two, not one, and that $A$ and $B$ and $C$ are three, etc.

Thus, while "a class as one may be a term of itself as many" (ibid., 102), a class as many of terms cannot be a member of itself since it is not 
a term. Indeed, regarding his paradox of the class of classes that are not members of themselves, Russell found that he could only conclude that "the classes which as ones are not members of themselves as many do not form a class-or rather, that they do not form a class as one, for the argument cannot show that they do not form a class as many" (ibid., 102).

\section{The Class as Many as a Plural Logical Subject}

A class as many, according to Russell, is "an object uniquely determined by a propositional function, and determined equally by any equivalent propositional function" ([POM], 515). As so determined, moreover, or so Russell claimed, a class as many of (say) individuals must be such that we can speak of it as one object-and not only with respect to its identity or diversity from other classes as many of individuals (which can, after all, be explained by the equivalence or nonequivalence of propositional functions), but also, and especially, as an object that can meanfully be said to be a member of a class as many of classes as many of individuals (cf. [POM], 516f). We must, in other words, be able to speak of classes as many as logical subjects (even if not as single logical subjects), since otherwise we could not speak of classes as many of classes as many, even when the former are of a higher logical type than the latter.

Now the notion of an object that Russell uses here to speak of a class as many as one, when in fact it is not one but many, should not be confused with the notion of an entity; for although every entity is an object, not every object, and in particular no class as many, is an entity. Of course, as Russell himself admits, "the fact that a word can be framed with a wider meaning than term raises grave logical problems" (ibid., 55); for how can that which is not a term and which cannot occur in a proposition as a single entity be a logical subject in the sense of being that which a proposition is about?

Russell's answer here is the conceptual linchpin of his first theory of logical types, since this theory is intended to apply to classes as many. It is, in his own words, "the fundamental doctrine upon which all rests" (ibid., 517); and it is briefly described as "the doctrine that the subject of a proposition may be plural, and that such plural subjects are what is meant by classes" (ibid.). The notion of an object, in other words, is intended by Russell to cover both plural and single logical subjects. 


\section{Logical Types and the Notion of a Logical Subject}

Less briefly, however, the doctrine Russell intends here is semantically based upon his first or early theory of denotation, and in particular on his theory of denoting concepts. These are in every case complex concepts derived from a class-concept (i.e., one ordinarily represented by a common noun) by means of one of the operations associated with the words 'all', 'every', 'any', 'a', 'some', and 'the'. Those in particular that denote classes as many are indicated by expressions of the form 'all $u$ 's', or ' $u$ 's' simpliciter, where ' $u$ ' itself stands for a class-concept. (On Russell's interpretation of the plural, 'all $u$ 's' and ' $u$ 's' are taken as synonymous (ibid., 72). Russell also uses the plural 'the', as in 'the inhabitants of London', 'the sons of rich men', etc., as a stylistic variant of 'all (the) $u$ 's'.)

A class-concept, it should be noted, does not itself denote, no less denote a class; and therefore it should not be confused with a denoting concept of which it may be a component. Thus, for example, while the classconcept man occurs in the proposition expressed by 'Socrates is a man', it occurs only as a component of the denoting concept $a$ man, which in turn denotes not a class but some particular man-although it is ambiguous as to which man is in fact denoted. In the proposition expressed by 'Socrates is one among men', on the other hand, it is not the class-concept man that occurs but its plural, men, which, as noted, is the same for Russell as the denoting concept all men; and which in this case does in fact denote the class (as many) of men. (A denoting concept, incidentally, such as men or all men, which unambiguously denotes an object is said by Russell to be a concept of that object, which of course, in the case of a class as many, is not to be confused with a class-concept [cf. p. 67].)

Class-concepts themselves, according to Russell, are either (1) predicates, i.e., concepts indicated by adjectives (ibid, 44); or (2) are derived somehow from predicates, as man is derived from human'; or (3) are obtained by an application of the notion such-that to a propositional function, such as is indicated e.g. by ' $x$ such that $\varphi x$ '. The plural denoting concept that contains this last class-concept as a component, and that denotes the class as many of objects satisfying the propositional function in question, is the concept indicated by ' $x$ 's such that $\varphi x$ ', which, as noted, is taken by Russell to be synonymous both with 'all (the) $x$ 's such that $\varphi x$ ' and 'the $x$ 's such that $\varphi x$ '.

Both predicates and propositional functions, accordingly, generate class-concepts, which in turn are components of denoting concepts, i.e., 
complex concepts corresponding to expressions of the form 'all $u$ 's, 'every $u$ ', 'any $u$ ', 'a $u$ ', 'some $u$ ', and 'the $u$ ', where ' $u$ ' itself stands for a class-concept; and denoting concepts themselves, it is clear, are the conceptual counterparts of (surface) grammatical subjects of English declarative sentences. Denoting concepts, in other words, are taken by Russell as occurring in propositions in a manner that at least semantically is completely analogous to the way that (surface) grammatical subjects occur in the English declarative sentences that express those propositions.

Now it is clear that what this theory requires is that singular and plural grammatical subjects are to be given parallel semantical analyses; and that in itself is not an implausible thesis (as witness its defense in Montague grammar). How that thesis is carried through, on the other hand, is another matter altogether; and Russell's own particular analysis, according to which there are both single and plural logical subjects just will not do. Thus, as Russell himself was led to comment in [1908], "even if there were such an object as 'all men', it is plain that it is not this object to which we attribute mortality when we say 'all men are mortal' " ([1908], 70). Moreover, since the object supposedly denoted by 'all men' is the class as many of men, and since, by the theory of logical types, a class as many can be a logical subject only of "propositions of a different kind from those in which its terms are subjects" ([POM], 105), then "all men are mortal' and 'Socrates is mortal' must express different kinds of propositions, which contradicts the thesis in question, viz., that singular and plural grammatical subjects are to be given parallel semantical analyses. In this regard, Russell's attempt to retain classes as many as plural logical subjects must be deemed a failure, which in fact is Russell's own later view of the matter.

\section{Propositional Functions as Nonentities}

Indeed, regardless of whatever initial plausibility that Russell's theory of denoting concepts might be thought to have (excluding, of course, the dubious notion of a plural logical subject), it was precisely this theory that Russell later rejected in his 1905 article 'On denoting'. Once rejected, moreover, Russell's options, as described in [1905], are either to consider classes only as single entities, or to somehow avoid the assumption that there are any classes at all; and as is made clear by the February, 1906 
note appended to the end of that article, Russell's choice is in favor of the latter alternative, which he also came to call the "no classes" theory.

Indeed, in a way, Russell's choice was already determined by his view in $[P O M]$ of propositional functions as nonentities. For, rightly or wrongly, on his own interpretation of the options available to him in 1905 , i.e., after the formulation of his new theory of denoting, Russell claimed that "the postulate of the existence of classes [as single entities] ... is exposed to the same arguments, pro and con, as the existence of propositional functions as separable entities distinct from all their values" ([1905], 154). That is, the problem of the One and the Many applies to propositional functions no less so than it does to classes; and if propositional functions are not single entities having an existence that is separable from the many propositions that are their values, then so too classes are not single entities having an existence that is separable from their many terms.

Now it is not prima facie absurd to think that a propositional function can be a single logical subject, i.e., that it can also occur in a proposition as a term of the form 'being an $x$ such that $\varphi x$ ', the way, e.g., the predicate (concept) buman can also occur as the term bumanity. That, moreover, would be what would obtain, at least in effect, if every propositional function were predicative in the sense of satisfying the following comprehension principle:

$$
\left(\mathrm{CP}^{*}\right) \quad(\exists F)(x)[F x \equiv \varphi x],
$$

where ' $(\exists F)$ ' is read as 'there is a predicate (concept) F' such that' (and where ' $F$ ' does not occur free in the formula indicated by ' $\varphi x$ '). ${ }^{3}$

On this point, however, Russell demurs. For as a result of his paradox of predication, i.e., his paradox stated in terms of predicates, Russell concluded that there can be no predicate (concept) corresponding to the propositional function indicated by ' $x$ is identical with a predicate that is not predicable of $x$ ' ([POM], 97). At least this propositional function, in other words, is not predicative in the above sense; and therefore $\left(\mathrm{CP}^{*}\right)$ is not in general logically valid.

No paradox would result even in this case, it should be noted, if Russell had not assumed that predicates are individuals, i.e., that a predicate can occur in a proposition as a term as well as a concept (though of course in no single occurrence can it occur both as a term and as a concept). For it 
is only by allowing a predicate to occur both as a term and as a predicate in the propositional function represented by ' $x$ is identical with a predicate that is not predicable of $x$ ' that a contradiction is forthcoming-on the additional assumption, of course, that the propositional function in question is predicative in the above sense, or on the more general assumption of the logical validity of $\left(\mathrm{CP}^{*}\right)$.

It is perhaps instructive in this last regard to compare Russell's response in $[P O M]$ to essentially the same argument from the point of view of the following comprehension principle:

(CP) $\quad(\exists \psi)(x)[\psi x \equiv \varphi x]$

where ' $(\exists \psi)$ ' is read as 'there is a propositional function $\psi$ such that' (and where ' $\psi$ ' does not occur free in the formula indicated by ' $\varphi x$ '). For this comprehension principle, unlike $\left(\mathrm{CP}^{*}\right)$, is easily seen to be logically valid on the basis of a trivial existential generalization of a universally quantified tautologous formula. (It is clear, of course, that for Russell "it is impossible to exclude variable propositional functions altogether" ([POM], 104), especially since "wherever a variable class or variable relation [in extension] occurs, we have admitted a variable propositional function, which is thus essential to assertions about every class or about every relation" [ibid.]). ${ }^{4}$

Thus, since (CP) is logically valid, it is true even of the propositional function indicated by ' $x$ is identical with a predicate that is not predicable of $x$ '. No paradox is forthcoming, however, since Russell does not assume that propositional functions are single entities, i.e., that they can exist separable from the many propositions that are their values. And though he need only not assume that all propositional functions are single entities, in $[P O M]$ his original "non-assumption" has the same status as a negative assumption: "the $\varphi$ in $\varphi x$ is not a separate and distinguishable entity: it lives in the propositions of the form $\varphi x$ and cannot survive analysis" (p. 88). Thus, since being a separable entity is the same as having the capacity of being a logical subject, there can be no proposition of the form $\psi(\varphi)$, no less one of either the form $\varphi(\varphi)$ or of the form not $-\varphi(\varphi)$; and therefore, according to Russell, the above contradiction is avoided by "the recognition that the functional part of a propositional function is not an independent entity" (ibid.).

By 1905, however, Russell's "non-assumption" is no longer identified 
with his negative assumption, and he is willing to consider at least the possibility of there being some propositional functions-though necessarily not all-that are separable, and therefore single, entities, i.e., the possibility that $\left(\mathrm{CP}^{*}\right)$ might be true of at least some propositional functions. It is this possibility, moreover, that he now associates with the existence of a class as one, i.e., with the existence of a class as "a new single entity" (over and above the many terms that are its members). Indeed, it is in fact this very association that explains the terminological basis for Russell's first, explicit definition of predicativity, viz., his [1905] definition that a propositional function is predicative if, and only if, it defines a class as one, i.e., if, and only if, it satisfies the following comprehension principle:

$$
\therefore(\mathrm{CP}-\in) \quad(\exists y)(x)[x \in y \equiv \varphi x]
$$

(where ' $y$ ' is an individual variable that does not occur free in the formula indicated by ' $\varphi x$ '). For if there is such a class respecting a given propositional function, then, according to Russell, the very condition expressed by ' $x \in y^{\prime}$ ' represents a predicate of $x$, in which case (CP*) is validated for that propositional function ${ }^{5}$; and, conversely, "the arguments which show that there is not always a class also show that there is not always a separable entity which is the propositional function (as opposed to its value)" ([1905], 145). That is, a propositional function for which (CP- $\in$ ) fails is one, according to Russell, for which $\left(\mathrm{CP}^{*}\right)$ also fails. (The resulting equivalence of $\left(\mathrm{CP}^{*}\right)$ with $(\mathrm{CP}-\epsilon)$, moreover, is in fact the basis of Russell's later attempt to justify the axiom of reducibility.)

Now it is precisely the possibility of there being some propositional functions, and therefore some classes as one, as separate, single entities that Russell takes the zig-zag and limitation of size theories as attempting to realize. ${ }^{6}$ Such an attempt, he maintains, "has the advantage of being more consistent with common sense, and of preserving more of Cantor's work; but it has, as yet, the disadvantage of great uncertainty and artificiality in detail, owing to the absence of any broad principle by which to decide as to which functions are predicative" ([1905], 164).

In the end, finding no such broad principle himself, Russell concluded that it is in fact safer to abstain from assuming that any propositional function has a separate existence, and, similarly, to abstain from assuming that there are any classes; and such abstinence is in fact what he generally means by the "no classes" theory. Unfortunately, however, Russell 
frequently confuses these "non-assumptions" with their negative assumptions, and in consequence he sometimes describes the "no classes" theory as the denial that there are any classes; and similarly he commonly reverts to his earlier $[P O M]$ view that assumes that no propositional function is or can be a single entity, separable from the many propositions that are its values.

\section{Propositions as Single Logical Subjects}

In claiming that a propositional function cannot exist as a single entity, separable from the many propositions that are its values, Russell means not only to make the negative claim that it is not any supposed separable existence of the propositional function that gives rise to the propositions that are its values, but also, and especially, the positive claim that it is in fact the existence of each of these propositions as single entities that gives rise to the propositional function. Thus, for example, if $\varphi(a)$ is a proposition and $x$ is any term, then "we can consider the proposition $\varphi(x)$, which arises from the substitution of $x$ in place of $a$ " and thereby "arrive at the class of all propositions $\varphi(x)$ " ([POM], 93). A unary propositional function, in other words, can be identified (or at least strongly associated) with "the class of all propositions which arise from the variation of a single term" (ibid.), and a similar identification can be made for propositional functions of more than one argument. This cannot be taken as a definition in $[P O M]$, however, since classes there are themselves identified as the extensions of propositional functions.

Now it is precisely this same circle of ideas, viz., (1) that of a proposition as a single entity, (2) that of the substitutional characterization of a propositional function as a class of similar propositions, and (3) that of a class as the extension of a propositional function, which together with his new 1905 theory of incomplete symbols, i.e., his new theory of denoting, led Russell to his 1906 substitutional theory of classes, i.e., the theory that characterizes the second major stage in the development of his theory of logical types. It was in this substitutional theory, moreover, that Russell briefly attempted to return to the pristine simplicity of the unrestricted variable and the univocity of being, and in which he assumed neither classes nor propositional functions to be values of any variable whatsoever.

Now it is fundamental to this theory that propositions are to be consid- 
ered as complex single entities, i.e., as terms that can occur as logical subjects of other propositions and yet as terms that contain terms that may have other terms substituted for them. It is through the occurrence of a proposition as a complex term of other propositions, in other words, that Russell first attempted to formulate his "no classes" theory; and in this regard it is perhaps appropriate that we briefly reexamine Russell's [POM] view of propositions as single entities, i.e., as logical subjects, before turning to the specifics of the 1906 substitutional theory.

One important point that should be noted regarding the notion of a proposition in $[P O M]$, and which does not apply to the notion of a proposition in the 1906 substitutional theory, is that in general a proposition is not a state of affairs, or at least not in the sense that a state of affairs can be about an individual if, and only if, that individual is a constituent of, i.e., occurs in, that state of affairs. Thus, e.g., in the proposition expressed by 'I met a man', it is the denoting concept a man, and not any one particular man, that occurs; and yet, "the proposition is not about the concept a man, but about something quite different, some actual biped denoted by the concept" ([POM], 47).

This distinction between being a logical subject in the sense of being what a proposition is about and being a logical subject in the sense of actually occurring in that proposition as a term is important to Russell in $[P O M]$, moreover, precisely because it allows him there the means for distinguishing when a concept occurs in a proposition as a term as opposed to when it occurs as a concept. Grammatically, of course, the two kinds of occurrences are distinguished in the corresponding sentences by means of a nominalizing transformation, such as when 'human' is transformed into 'humanity' or 'is' is transformed into 'being'. The nominalizing transformation, moreover, is not to be thought of in the manner of Frege, i.e., as somehow correlating a self-subsistent entity or term with a concept that in itself is not self-subsistent. That is, concepts differ from terms that are not concepts, according to Russell, "not in respect to self-subsistence, but in virtue of the fact that, in certain true or false propositions, they occur in a manner which is different in an indefinable way from the manner in which subjects or terms of relations occur" (ibid., 46).

This "indefinable way", moreover, in which a concept occurs in a proposition as a concept, and in which terms in general cannot so occur, can be identified in the case of a predicate (or adjectival concept) with the 
latter's occurrence as a component of a denoting concept; for, as already noted, a denoting concept does not occur in a proposition as a term or logical subject but as a concept that denotes the term or logical subject (or one of the logical subjects) that that proposition is about.

Russell himself tended to obscure this point, unfortunately, by sometimes distinguishing predicates from class-concepts (e.g., human from man), and by then taking the latter as somehow derived (in some unexplained manner) from the former. On our interpretation, however, it would suit Russell's purposes in [POM] better if we took the common noun class-concept as basic and the adjectival concept as being itself an el- \{ liptically expressed class-concept corresponding to the adjectival modif- ) cation of a common noun. Thus, e.g., 'Socrates is human' would really be elliptical for 'Socrates is a (hu)man (being)'; and the fact that human occurs in the proposition expressed by this sentence "in a different way from that in which it occurs when it is called bumanity" (ibid., 45 ) is then explained (on the basis of Russell's own theory of denoting concepts) by its occurring therein as a component of the denoting concept $a(b u) m a n$ (being). In this way, accordingly, both a predicate (or adjectival concept) and a (common noun) class-concept can be said to occur in a proposition as a concept and not as a term when, and only when, the occurrence in question is a component of a denoting concept (and where ellipsis has been eliminated).

A similar general reduction to denoting concepts does not apply to verbal concepts, however, or at least not to the extent that "one verb, and one only, must occur as verb in every proposition" (ibid., 52); for as so occurring, a verbal concept must not only occur in that "indefinable way" that distinguishes concepts from other terms, but it must do so without also occurring as a component of a denoting concept. Every proposition, in other words, must contain a verbal concept that in its occurrence as a concept combines the constituents of the proposition into the entity that is that proposition, but which in just that regard cannot so occur as a component of a denoting concept (cf. [POM], 50).

The restriction to a single verbal concept that occurs in each proposition as described above applies, it should be noted, to compound propositions, i.e., to propositions containing other propositions as constituents, no less so than to uncompounded propositions. Thus, e.g., the single verbal concept combining the constituents of the proposition expressed by 
'If Socrates is human, then Socrates is mortal' is the concept of (material) implication, since the same proposition, according to Russell, is also expressed by 'Socrates's being human (materially) implies Socrates's being mortal'. Here, of course, it is Russell's view that the two propositions expressed by 'Socrates is human' and 'Socrates is mortal' occur in the compound proposition as terms, and not as asserted propositions; and thus, in this regard, he is led to distinguish between the occurrence of a proposition as actually asserted from its occurrence when considered merely as a complex concept, which he also calls a propositional concept (cf. [POM], section 38). (It is in order to respect this distinction, incidentally, that Russell also needs the notion of therefore as well as the notion of (material) implication; for "when we say therefore, we state a relation which can only hold between asserted propositions, and which thus differs from implication" [ibid., 35]).

Grammatically, as the above example indicates, a propositional concept is denoted, according to Russell, by a nominalized sentence in which the verb of the original sentence has been transformed into a verbal noun (which is always either the infinitive or gerund of that verb). It is clear, moreover, at least to Russell, "that the concept which occurs in the verbal noun is the very same as that which occurs as verb" (ibid., 48), and that therefore every verbal concept "may also be made into a logical subject" (ibid., 52).

The situation appears to be more problematic for propositional concepts, however, at least according to Russell, for whom "there appears to be an ultimate notion of assertion [in a logical as opposed to a psychological sense], given the verb, which is lost as soon as we substitute a verbal noun, and is lost when the proposition in question is made the subject of some other proposition" (ibid., 48). Moreover, since this ultimate notion of assertion appears to be none other than the truth of a proposition (cf. $[P O M], 504)$, it would seem to follow then that that "indefinable way" in which a verbal concept occurs in a proposition that in fact is true is somehow so essential to that proposition's truth or assertibility (in the logical

$\rightarrow$ sense) that "the contradiction which was to have been avoided, of an entity which cannot be made a logical subject, appears to have become inevitable" (ibid., 48).

Indeed, it might even seem to be this same inevitability that Russell re- 
turns to in Appendix B, i.e., in his first theory of logical types. For since every object of whatever logical type is identical with itself, then the number of propositions asserting the self-identity of an object with itself must be "as great as that of all objects absolutely" (ibid., 526). However, since the propositional concept, as a single logical subject, "appears to be always an individual" (ibid.), and since, by Cantor's theorem, there are more classes of individuals than there are individuals, then, accordingly, there must be more objects absolutely than there are propositions. "The only method of avoiding this difficulty," according to Russell, is "to deny that propositional concepts are individuals; and this seems to be the course to which we are driven" (ibid.).

Appearances to the contrary, however, Russell is not yet prepared to give up his view of propositions as single entities, i.e., as single logical subjects; and the notion of assertion that he earlier took to be ultimate "in a logical as opposed to a psychological sense" (ibid., 49), he is now prepared instead to discard. "The propositional concept," he finally concludes, "seems, in fact, to be nothing other than the proposition itself, the difference being merely the psychological one that we do not assert the proposition in the one case, and do assert it in the other" (ibid, $526 \mathrm{f}$ ).

The upshot, accordingly, at least as far as Appendix B is concerned, is that propositions are indeed single logical subjects, but of a type different and disjoint from that of individuals. Both are single entities, but both cannot be values of a single unrestricted variable, i.e., of a variable representing a univocal sense of being, the way they had been throughout the bulk of $[P O M]$. How this difference between propositions and other terms as single entities is to be accounted for otherwise, however, i.e., otherwise then as values of different types of variables for logical subjects, is left unexplained by Russell in [POM]. It is clear, moreover, that it was not a solution with which he was satisfied, since it is precisely this distinction between propositions and other single entities that he came to reject in his 1906 substitutional theory of classes.

\section{The Substitutional Theory of Classes}

In his 1906 substitutional theory, Russell returned once again to his earlier pristine view of a single type of unrestricted variable and of a univocal 
sense of being that is shared by all logical subjects. Neither classes nor propositional functions are assumed to be values of this unrestricted variable, moreover, and in that regard they are not assumed to be entities of any kind whatsoever. With his new theory of denoting and of incomplete symbols in hand, Russell now proposes to analyze statements purportedly about classes into statements about propositions (as complex terms) and about the terms that might occur within them through the process of substitution. The resulting theory, Russell claims, "affords what at least seems to be a complete solution of all the hoary difficulties about the one and the many; for, while allowing that there are many entities, it adheres with drastic pedantry to the old maxim that, "whatever is, is one' " ([1906a], 189).

The fundamental principle from which the theory starts, according to Russell, "is that, in any sentence, a single word, or a single component phrase, may often be quite devoid of meaning when separated from its context" (ibid., 165); and "in such a case, if the word or phrase is wrongly assumed to have an independent meaning, we get what may be called a 'false abstraction', and paradoxes and contradictions are apt to result" (ibid.). Classes, furthermore, are precisely such "false abstractions."

To explain his contention, Russell introduces the notations ' $p(x / a)$ ! $q$ ' and ' $p / a ; x ! q$ ' as alternative abbreviations of ' $q$ results from $p$ by substituting $x$ for $a$ in all those places (if any) where a occurs in $p$ '. (The alternative notations are for different lines of development.) All the letters that occur here, incidentally, are of a single logical type-there being only the type of the unrestricted variable-and they all have single entities as their values, i.e., they all range over all entities whatsoever. Of course, "it is conve-

$\rightarrow 1$ nient to think of $p$ (and therefore $q$ ) as propositions, but this is not essential; all that is essential is that $p$ should be a name of a genuine entity and not a mere phrase like 'the King of France' or 'the King of England' " (ibid., 168).

Now it should be noted that the notion of substitution that Russell has in mind here is intended to apply to entities and not to the symbols that stand for those entities. Thus, taken primitively in this way, Russell is able to define the identity of $x$ with $y$ as $x$ 's being the result of substituting $y$ for $x$ in $x$ :

$$
(x=y)=\mathrm{df} x(y / x) ! x .
$$


The exclusion from, or non-occurrence of, one entity in another, where the latter is typically (but apparently not always ${ }^{7}$ ) a proposition, is defined by Russell as follows:

$$
a \exp =\mathrm{df}(x) \cdot p(x / a) ! p .
$$

That is, ' $a$ does not occur in $p$ ' means 'for every entity $x$, the result of substituting $x$ for $a$ in $p$ is $p$ '; and, accordingly, ' $a$ occurs in $p$ ' is then definable as the negation of ' $a$ does not occur in $p$ '. It is clear of course that ' $a$ occurs in $p$ ' is taken to be synonymous with ' $a$ is a constituent of $p$ '.

In regard to the expressions ' $p(x / a)$ ' and ' $p / a ; x$ ' that occur in the more complex notions described above, and that, when so separated, Russell takes as alternative abbreviations of 'the result of substituting $x$ for a in $p$ ', the component ' $(x / a)$ ' of the first is called a substitution, and the component ' $p / a$ ' of the second is called a matrix. Of course, taken out of context in this way, these components are incomplete expressions and "are wholly devoid of meaning by themselves and only become significant as parts of appropriate propositions" (ibid., 170). The matrix ' $p / a$ ' in particular is merely a stylistic variant of the incomplete phrase 'the result of replacing $a$ in $p$ by'; and in that regard is not, nor can it be, the name of an entity.

Nevertheless, notwithstanding its being an incomplete expression, it is precisely the matrix ' $p / a$ ' that Russell proposes to take as representing a class. Thus, according to Russell, ' $x$ is a member of the class $p / a$ ' is to be interpreted as 'the (proposition that is the) result of replacing $a$ in $p$ by $x$ is true'. Any two entities $p$ and $a$, in other words, "define a class, namely $p / a$, and $x$ is a member of this class if $p / a ; x$ is true. (If $p$ does not contain $a$, the class contains everything if $p$ is true, and nothing if $p$ is false)" (ibid., 172). ${ }^{8}$ Thus, although classes are not assumed to be values of the unrestricted variable, all statements about classes can be analyzed, according to Russell, into statements about pairs of entities, one of which is usually a proposition and the second of which is a term occurring in that proposition.

In regard to propositional functions, it will be remembered that even in $[P O M]$ these were not assumed to be values of the unrestricted variable, the way, e.g., classes (as ones) were. A propositional function, as noted in section 5 above, was to be identified somehow with the class of propositions that were its values, though this of course was not to be taken as a definition since classes were themselves identified as the extensions of 
propositional functions and quantification over classes in that sense presupposed quantification over propositional functions. How quantification with respect to propositional functions was to be understood otherwise, however, especially since they were not taken as entities of any kind whatsoever, was left unexplained; and it is this situation that Russell now attempts to rectify, or rather to cancel.

A propositional function, Russell continues to insist, "is nothing at all without some argument" ([1906a], 171), in which case it is none other than the proposition that is its value for that argument. In this regard, and unlike the problematic situation in $[P O M]$, "we can never say, of any formula containing a variable function, that it holds 'for some value of $\varphi$ ', because there is no such thing as $\varphi$ and therefore there are no 'values of $\varphi$ '" (ibid.). There are only entities, in other words, i.e. single logical subjects; and it is only with respect to entities as values of the unrestricted variable that any form of referential quantification is to be deemed at all meaningful.

Nevertheless, "by the help of matrices," Russell now claims, "one can almost always express what is substantially the same thing" (ibid., 172); and the limiting cases where matrices will not do this "are precisely those that lead to contradictions" (ibid.). Matrices, in other words, may be used to represent propositional functions as well as classes, or at least those propositional functions that do not lead to contradiction. Thus, for example, in the place of an expression of the form ' $\varphi x$ ' we can use an expression of the form ' $p / a ; x$ ' instead; and, similarly, in the place of 'all values of $\varphi$ ' we can use 'all values of $p$ and $a$ ' instead. In this way, according to Russell, while we cannot say ' $x$ is identical with $y$ when, and only when, $\varphi x$ implies $\varphi y$ for all values of $\varphi$ ', we can instead say ' $x$ is identical with $y$ when, and only when, $p / a ; x$, implies $p / a ; y$ for all values of $p$ and $a^{\prime}$. (Actually, however, for reasons yet to be explained, no proposition in the sense intended here by Russell can contain an apparent variable, and therefore the expression ' $p / a ; x$ ' really corresponds not to ' $\varphi x$ ' as an unqualified expression for a propositional function, but to the $[P M]$ notation ' $\varphi ! x$ ' for predicative propositional functions (of individuals) in the sense of *12).

Propositional functions of two arguments can also be represented in this notation; e.g., ' $p /(a, b) ;(x, y)$ ' is to be taken as an abbreviation of 'the result of simultaneously substituting $x$ for $a$ and $y$ for $b$ in $p$ '. Similarly, 
propositional functions of three arguments can be represented by ' $p /$ $(a, b, c) ;(x, y, z)$ ', and so on for propositional functions of any finite number of arguments. (It should be noted once again, however, that with respect to the later $[P M]$-notation, these matrices can only represent predicative propositional functions of individuals).

Now a matrix is also to be typed, according to Russell, and its type will depend on the number of terms involved in the simultaneous substitution characterizing that matrix. Thus, since a matrix of the form ' $p / a$ ' involves the substitution of a single term, it is said to be of the first type; and since a matrix of the form ' $p /(a, b)$ ' involves the similtaneous substitution of two terms, it is said to be of the second type; and so on for matrices of all finite types. (The typing of matrices, it should be noted, is to be carried out in the metatheory, since, strictly speaking, there are neither variables nor entities corresponding to the type of a matrix.)

The typing of matrices is to correspond, according to Russell, to his earlier (homogeneous) typing of classes in Appendix B of [POM]. Thus, whereas a class of entities is to be represented by a matrix of the first type, a class of classes of entities is to be represented by a special type of matrix of the second type, viz., one of the form ' $q /(p, a)$ ', where the first term of the substitution is a proposition; and a class of classes of classes of entities is then to be represented by a special type of matrix of the third type, and so on ad infinitum.

A matrix of the form ' $q /(p, a)$ ', for example, is to represent a class of classes of entities, according to Russell, "if, for all values of $r, c, r^{\prime}, c^{\prime}$, provided $r / c=r^{\prime} / c^{\prime}$, then $q /(p, a) ;(r, c)$ is equivalent to $q /(p, a) ;\left(r^{\prime}, c^{\prime}\right)$ " ([1906a], 176). The identity formula ' $r / c=r^{\prime} / c^{\prime}$ ' is understood here as an abbreviation for the equivalence of $r / c ; x$ with $r^{\prime} / c^{\prime} ; x$, for all entities $x$; and its inclusion above is to make sure that "the truth or falsehood of $q$ l $(p, a) ;(r, c)$ does not depend on $r$ and $c$ separately" (ibid.). Thus, assuming that ' $q /(p, a)$ ' rcpresents a class of classes of entities, 'the class $r / c$ is a member of the class of classes $q /(p, a)$ ' is to be interpreted, according to Russell, as 'the (proposition that is the) result of simultaneously replacing $p$ in $q$ by $r$ and $a$ in $q$ by $c$ is true'.

Finally, as an example of a class of classes of entities, consider the matrix ' $(x) . \sim(p / a) ; x /(r, c)$ ', which, because it involves the simultaneous substitution of two terms, is a matrix of the second type, i.e., it is a matrix of the form ' $q /(r, c)$ '. It is clear of course that the statement expressed here by 
' $(x) . \sim(p / a) ; x$ ' says in effect that the class $p / a$ is empty. It is also clear that if $r / c=r^{\prime} / c^{\prime}$, i.e. if

$$
(x)\left[r / c ; x \equiv r^{\prime} / c^{\prime} ; x\right],
$$

then the class $r / c$ is empty if, and only if, the class $r^{\prime} / c^{\prime}$ is also empty, i.e. then

$$
(x) . \sim(p / a) ; x /(r, c) \equiv(x) . \sim(p / a) ; x /\left(r^{\prime}, c^{\prime}\right) .
$$

Thus the matrix ' $(x) . \sim(p / a) ; x /(r, c)$ ' fulfills Russell's definition and therefore represents a class of classes of entities; and in fact the class of classes it represents is defined by Russell to be the cardinal number 0 .

There are difficulties with this interpretation of classes, however, not the least of which is Russell's old problem with Cantor's theorem that there are more classes of entities than there are entities. The problem here of course is how this could be so if each class of entities is determined by a pair of entities $p, a$, and if, furthermore, the number of entities is infinite-a fact, incidentally, that Russell claimed to prove in [1906b]. ${ }^{9}$

Russell's "solution" to this problem, unfortunately, is no solution at all; for "any two entities $p, a$," he says, "define a class $p / a$; but a pair of entities is not one entity, and the number of pairs of entities may therefore, without the slightest contradiction, be greater than the number of entities" ([1906a], 180). This would be so, however, only if the number of entities were finite; and having "proved" that the number of entities is infinite, Russell could have gone on to prove that the number of pairs of entities is the same as the number of entities.

\section{Propositions versus Statements in the Substitutional Theory}

Shortly after Russell first posed the question in [1905] as to which propositional functions are predicative in the sense of satisfying (CP- $E$ ), Poincare responded with an answer to the effect that those and only those propositional functions are predicative that do not involve a vicious circle. ${ }^{10}$ Russell, in his reply to Poincare, agreed that "the clue to the paradoxes is to be found in the vicious-circle principle" ([1906b], 198), but he went on to claim that such an answer could be formally realized only in a theory resembling his substitutional "no-classes" theory. The vicious cir- 
cle suggestion itself he then reformulated as the principle that "whatever involves an apparent variable must not be among the possible values of that variable" (ibid.).

There is a difficulty in applying this principle in the substitutional theory, however; for, as already noted, it is a fundamental assumption of that theory that whatever is-and that includes all propositions-is a value of the unrestricted variable, i.e. of the one type of variable that occurs in the theory and that as such represents the univocal sense of being that is shared by all logical subjects. Consequently, since by the viciouscircle principle "whatever involves all must not be one of the all which it involves" (ibid., 204), it follows that "to avoid vicious circles, we need a meaning of proposition according to which no proposition can contain an apparent variable" (ibid.).

We shall not ourselves dwell here on the question that arises regarding the sense in which a variable, whether apparent or real (i.e., whether bound or free, respectively) might be said to occur in a proposition (as opposed to a sentence that expresses that proposition). For whatever the explanation, ${ }^{11}$ it is clear that many of the propositions that Russell had formerly allowed, and apparently needed in his logical reconstruction of mathematics, were now to be excluded. The logical analysis of mathematical propositions that seem to involve an apparent variable could still be secured, according to Russell, "by deciding that a statement about all ... is really an affirmation of an ambiguous one of the several propositions got from particular cases" (ibid.). Thus, for example, "if we state: 'Whatever $x$ may be, $x=x$, we are stating an ambiguous one of the propositions of the form ' $x=x$ '" (ibid.); and here it should be noted that "though we have a new statement, we do not have a new proposition" (ibid.). That is, the same result is to be secured, according to Russell, by distinguishing propositions from the statements in which they might be affirmed.

Russell does not himself explain what it is that he takes a statement to be, though apparently what he has in mind here is a linguistic or mental act of affirming (or denying), which together with a content (or state of mind) can in some sense be said to contain, or not to contain, an apparent variable (as characterized, e.g., by the sentence uttered in an overt performance of that act). ${ }^{12}$ A proposition, on the other hand, is not a statement but is what is affirmed by a statement, and in particular is "confined to what is affirmed by a statement containing no apparent variable" (ibid., 
207). A proposition in this sense, moreover, is an objective truth (i.e., a "fact" [ibid.]) or an objective falsehood, and differs in that regard from the relative truth or falsehood of a statement in which that proposition might be affirmed.

In applying this distinction between statements and propositions to the paradox of the liar, Russell suggests that the simplest interpretation of the statement expressed by someone who says 'I am lying' is the following: 'There is a proposition $p$ that I am affirming and that is false'. Interpreted in this way, the statement can be said to contain an apparent variable; and therefore, according to Russell, the statement is simply false without at the same time being true. That is, the statement is false not because the person making it "is stating a true proposition, but, because, though he is making a statement, he is not stating a proposition. Thus when he says he is lying, he is lying, and the inference that he must therefore be stating what is true fails" (ibid.).

The liar, Russell claims, "cannot mean: 'I am now making a statement which is false', because there is no way of speaking of statements in general: we can speak of statements of propositions, or statements containing one, two, three ... apparent variables, but not of statements in general" (ibid.).

The law of excluded middle in the form 'every proposition is either true or false' is another example of a statement that, because it contains an apparent variable, does not state a proposition. However, unlike the statement of the liar, the law of excluded middle is a true statement in the sense that "all the propositions which the statement ambiguously denotes are true" (ibid., 208). But it should also be noted here, however, that just as we cannot speak of statements in general, so too, "as applied to statements the meaning of the word true varies as the number of apparent variables in the statement varies" (ibid.).

Accordingly, just as different types of matrices can be distinguished depending on the number of terms involved in a specified simultaneous substitution, so too different types of statements can be distinguished depending on the number of nested apparent variables occurring in those statements. ${ }^{13}$ The different notions of (relative) truth and falsehood, as applied to statements, would then generate another hierarchy different from the hierarchy of matrices; and whereas the latter hierarchy is required to solve 
"all paradoxes concerning classes and relations [in extension]" (ibid., 204), the hierarchy of statements, with those affirming propositions as objective truths or falsehoods at the bottom, is required, according to Russell, to solve all intensional paradoxes, such as that of the liar or those involving the notion of definition (as in, e.g., 'the least indefinable ordinal' or 'the least integer not nameable in fewer than nineteen syllables').

Neither of these hierarchies, it should be noted, nor the typing of matrices and statements involved in their generation, correspond to anything in reality, i.e., none of the items occurring in these hierarchies are to be taken as values of the unrestricted variable. The reason, according to Russell, is that in order "to reconcile the unrestricted range of the variable with the vicious-circle principle, which might seem impossible at first sight, we have to construct a theory in which every expression which contains an apparent variable (i.e. which contains such words as all, any, some, the) is shown to be a mere façon de parler, a thing with no independent reality" (ibid., 206). Accordingly, "if (say) $\varphi x$ is true for every value of $x$, it will be not true, but meaningless, if we substitute for $x$ any expression containing an apparent variable. And such expressions exclude all descriptive phrases (the so-and-so), all classes, all relations in extension, and all general propositions [sic], i.e., all propositions [sic] of the form ' $\varphi x$ is true for all (or some) values of $x$ " " (ibid.).

Needless to say, but such a theory is obviously much too stringent for the purposes of mathematics, as Russell himself noted in his discussion of the principle of mathematical induction, i.e., the principle that any property that belongs to 0 , and that belongs to the successor of any number having the property, belongs to all finite numbers. ${ }^{14}$ Thus, for example, the phrase 'any property' that occurs in the statement of this principle "must, if the vicious-circle fallacy is to be avoided, be restricted to properties $\varphi x$ which can be stated without introducing any apparent variables" (ibid., 211). ${ }^{15}$ Of course, this restricton "will render most of the usual uses of induction fallacious; and in other ways it will destroy many pieces of ordinary mathematical reasoning" (ibid.).

The mitigating axiom that Russell proposes in order to overcome this difficulty is "the assumption that every statement containing $x$ and an apparent variable is equivalent, for all values of $x$, to some statement $\varphi x$ containing no apparent variable" (ibid., 212). (It is this assumption that is re- 
formulated in $* 12$ of $[P M]$ as the axiom of reducibility.) Equivalence here, of course, means only that the statement forms in question determine the same extension, and not that the statements that are expressed are themselves the same. Such an equivalence, when applied to extensional contexts, will justify the interchange of statement forms containing an apparent variable with statement forms containing no apparent variables; and therefore, on the assumption that the statement forms of pure mathematics are always extensional, all the usual uses of mathematical induction and the many pieces of ordinary mathematical reasoning can be easily retrieved. The same equivalence, on the other hand, will not justify the interchange of statement forms within nonextensional contexts; and in that regard the axiom will not cancel out the solution that the hierarchy of statements provides for the intensional paradoxes.

There is a limitation in the application of this axiom even in extensional contexts, however; and that is that a statement form may be interchanged with another, on the basis of their material equivalence, only . where the latter occurs assertorically, i.e. otherwise than as a single logical subject, and therefore otherwise than where it occurs as a term in a matrix. ${ }^{16}$ Of course, an axiom of extensionality to the effect that equivalent statement forms have the same, rather than merely equivalent, values would suffice for the latter interchange as well, i.e., for their interchange as single logical subjects; but that, given bivalence, would have the consequence that there really are only two statements, and therefore only two propositions, viz., a single objective truth and a single objective falsehood. It would also nullify the hierarchy of statements and Russell's proposed solutions of the liar and other intensional paradoxes.

With the limitation in effect, however, it is not clear how Russell can actually carry through his program of representing numbers as classes of classes of entities-i.e., by matrices of the second type, the way, e.g., 0 is represented by ' $(x) . \sim(p / a) ; x /(p, a)$ ' - that are themselves members of classes of classes of classes of entities, the way 0 is a member of the class of inductive cardinal numbers. For in order to carry through such a representation of numbers, Russell would perforce need to assume the interchange or substitution of statements containing apparent variables for statements not containing apparent variables in positions suitable only for single logical subjects. 


\section{The 1908 Theory of Logical Types}

Although the distinction between statements and propositions is not eliminated in [1908], nevertheless it is clear that a proposition is now affirmed (or denied) by each and every statement, regardless of whether the latter contains an apparent variable or not. In particular, according to Russell, "whatever we suppose to be the totality of propositions, statements about this totality generate new propositions which, on pain of contradiction, must lie outside the totality" ([1908], 62).

Propositions, moreover, though they are still the objective truths and falsehoods of the world, may now also contain apparent variablesthough Russell nowhere explains how this may be possible. ${ }^{17}$ Accordingly, by the vicious circle principle, just as there can be no way of speaking of statements in general, so too "there must be no totality of propositions, and 'all propositions' must be a meaningless phrase" (ibid.).

The propositions affirmed in the earlier substitutional theory remain a legitimate totality, however, and are in fact the basis for the generation of all other types of proposition. These are now described merely as those propositions that themselves contain no apparent variables and are referred to by Russell as elementary propositions. The terms, as opposed to the concepts, that occur in an elementary proposition also form a legitimate totality and are referred to by Russell as individuals. Individuals form the first or lowest logical type.

Although individuals are logical subjects par excellence, it does not follow that they are the only logical subjects; and, indeed, propositions remain logical subjects, or single entities, in Russell's 1908 theory of types, even if they must now be construed as belonging to different logical types. In this regard, it should be noted, each logical type is to be represented by a variable whose values are all and only single entities of that logical type. Thus, for the logical subjects of the first or lowest logical type, there are individual variables; and propositions that either contain no apparent variables or that have only individuals as values of the apparent variables they do contain are said by Russell to be first-order propositions. These propositions form the second logical type.

A proposition that contains an apparent variable, according to Russell, "presupposes others from which it can be obtained by generalization" 
(ibid., 75); and in that regard, therefore, all generalized propositions, i.e. those containing an apparent variable, presuppose elementary propositions. The process of generalization, moreover, is essentially the process involved in the method of substitution already described in the earlier substitutional theory-except that now propositions are to be typed (as well as statements), and they are in that regard to be taken as values only of variables of the appropriate logical type.

Thus, for example, if $p$ is an elementary proposition and $a$ is an individual occurring in $p$, then the generalization of $p$ with respect to $a$ is a firstorder proposition, represented by ' $(x) .(p / a) ; x$ ' and affirmed by a statement asserting $p / a ; x$, for all individuals $x$, i.e., for all values of the individual variable ' $x$ '. (Lower case Latin letters are used by Russell as variables of the lowest logical type occurring in a given context. The letters ' $x$ ', ' $y$ ', ' $z$ ', etc., will therefore not always have individuals as their values.)

The process of generalization need not always be only with respect to an individual, however; and in particular it may also be applied to any proposition as a single entity of a given logical type. Thus, since firstorder propositions form a logical type, i.e., a legitimate totality of entities or single logical subjects with respect to which generalization is meaningful, then, according to Russell, we can "form new propositions in which first-order propositions occur as apparent variables. These we will call second-order propositions" (ibid., 76). These propositions form the third logical type.

In general, accordingly, by means of the above process of generalization, "the $n+1$ th logical type will consist of propositions of order $n$, which will be such as contain propositions of order $n-1$, but of no higher order, as apparent variables. The types so obtained," or at least so Russell claims, "are mutually exclusive, and thus no reflexive fallacies are possible so long as we remember that an apparent variable must always be confined within some one type" (ibid., 76f).

The apparent variables of the theory as described so far, it should be noted, are those for individuals as the first or lowest type of logical subject and those for the propositions of different orders that are generated from the elementary propositions by the process of generalization. The theory resembles in this regard the earlier substitutional theory, except that now propositions can contain apparent variables and are themselves values only of the propositional variables of different orders. It is not sur- 
prising, therefore, to find Russell maintaining once again that the expression for a variable propositional function or variable class is to be considered as a mere façon de parler, and that neither propositional functions nor classes are to be considered as having an independent reality.

Nevertheless, according to Russell, "although it is possible to replace functions by matrices, and although this procedure introduces a certain simplicity into the explanation of types, it is technically inconvenient" (ibid., 77). That is, "in practice, a hierarchy of [propositional] functions is more convenient than one of propositions" (ibid.). Thus, instead of using ' $p / a$ ' and ' $p / a ; x$ ', or ' $p /(a, b)$ ' and ' $p /(a, b) ;(x, y)$ ', etc., where only individuals and propositions of various orders may occur as the values of the apparent variables, Russell proposes-but supposedly only for the purposes of technical convenience-that, we use ' $\varphi a$ ' and ' $\varphi x$ ', or ' $\varphi(a, b)$ ' and ' $\varphi(x, y)$ ', etc., respectively, instead. Of course, Russell notes, "in order that $\varphi$ may be legitimate as an apparent variable, it is necessary that its values should be confined to propositions of some one type" (ibid.).

In this regard, accordingly, since a propositional function both presupposes and is determined by the totality of propositions that are its values, a first-order propositional function is one whose values are always firstorder propositions; and, in general, an $n$ th-order propositional function is one whose value is always an $n$ th-order proposition. The logical type of a propositional function is then determined by the type of its values and the number and type of its arguments.

In terms of the hierarchy of orders, a propositional function of one argument is now said to be predicative if its order is the next above that of its arguments; and a propositional function of several arguments is said to be predicative if it is predicative with respect to one of its arguments when the values of the others are assumed to be fixed. To express the fact that $\varphi$ is a predicative function of $x$ (where $x$ may be of any logical type), Russell uses ' $\varphi ! x$ ', rather than the unexclaimed ' $\varphi x$ '.

It is important to note here, incidentally, that in allowing propositional functions to occur as values of an apparent variable, Russell also means to allow them to occur as single logical subjects, i.e., as arguments themselves of propositional functions of a higher order. In this regard, Russell identifies or conflates the notion of being the value of an apparent variable (of a given logical type) with that of being a single entity (of that logical type) in the sense of having the capacity to be the logical subject of a 
proposition (of the relevant type). Such an identification, to be sure, was already made in the 1906 substitutional theory (where to be was to be a value of the one type of unrestricted variable), but special variables for propositional functions were not allowed in that theory; and whereas such variables were allowed in $[P O M]$, propositional functions were assumed there to be nonentities, i.e., they could not themselves be the logical subject of a proposition. The present 1908 theory resembles the 1906 theory in the identification or conflation of these notions, accordingly, while yet, according to Russell, its application is not intended to commit us to the existence of propositional functions as single entities, i.e., as single logical subjects.

Needless to say, but it is essential to Russell's analysis of mathematics that propositional functions must be allowed to occur as single logical subjects, i.e., as arguments of higher order propositional functions; and he explicitly introduces for the latter purpose the notation ' $\varphi \hat{x}$ ', which is to denote the function itself, as opposed to ' $\varphi x$ ', which denotes not the function but a proposition as an indeterminate value of that function. In this regard, Russell will now allow into the theory not only propositions of the form ' $(\exists \varphi) \varphi$ ! $a$ ' but also propositions of the form ' $f$ ! $(\varphi ! \hat{x})$ '.

Having propositions of this latter form, we have said, is essential to Russell's analysis of mathematics, and in particular to his analysis of statements about classes. This is so because a statement about a class, according to Russell, is essentially a statement about the extension of some propositional function; and the latter, assuming that propositional functions can be single logical subjects, can be interpreted as a statement about some (or preferable any) propositional function materially equivalent to that propositional function. Thus, reading ' $\hat{z}(\mid y z)$ ' as 'the class defined by $\psi$ ', Russell gives the following contextual analysis for statements in which a class appears as a single logical subject (ibid., 89):

$$
f\{\hat{z}(\mid, z)\}=\mathrm{d} f(\exists \varphi)((x)[\varphi ! x \equiv \| x] \& f\{\varphi ! \hat{z}\}) .
$$

And, of course, in this analysis, it is essential that a propositional function can occur as a single logical subject of the analysans. ${ }^{18}$ In terms of this analysis and the assumption that every class is the extension of a propositional function of some logical type, quantification over classes can be contextually defined as follows:

$$
(\alpha) \varphi\{\alpha\}=\mathrm{d} f(\psi) \varphi\{\hat{z}(\psi ! z)\}
$$




$$
(\exists \alpha) \varphi\{\alpha\}=\mathrm{d} f(\exists \Downarrow) \varphi\{\hat{z}(\psi ! z)\}
$$

(where, of course, it is understood that in order for ' $\alpha$ ' to be used as an apparent variable, it is necessary that its values should be confined in any given context to propositional functions of some one type).

It is now clear how, by means of the contextual definitions given above, we are to understand Russell's claim that expressions for classes are incomplete symbols or mere façon de parler and that classes themselves need not be assumed to exist as values of any of the variables of the primitive framework of the theory. The question that remains, however, is how any such similar analysis is to be given for propositional functions, since, according to Russell, they too are not assumed to exist as values of the different types of variables in the primitive framework of the theory.

That an expression for a propositional function is somchow to be replaced by a matrix that contains only variables for individuals and propositions of different orders is clear from what Russell has already said in this regard. Moreover, with respect to purely assertoric occurrences of a propositional function, i.e., its occurrence otherwise than as a single logical subject, Russell, as we have already noted in section 6 , has given at least an example of how a variable for propositional functions is to be eliminated in favor of matrices and variables only for individuals and propositions of different orders. For example, in place of his [1908] definition of identity (p. 85):

$$
x=y=\mathrm{d} f(\varphi)(\varphi ! x \supset \varphi ! y)
$$

Russell could have used instead:

$$
x=y=\mathrm{d} f(p)(z)(p / z ; x \supset p / z ; y),
$$

where ' $x$ ', ' $y$ ', ' $z$ ' are variables of the same logical type in the primitive framework of the theory-i.e., they are either individual or propositional variables of the same order-and ' $p$ ' is a propositional variable of the next higher type.

The real difficulty in Russell's program, however, is not the elimination of variables for propositional functions that occur only assertorically; rather, it is the analysis in general of the occurrence of a propositional function as a single logical subject of a proposition or propositional function of higher order. And, to the extent that the above analysis of classes 
in terms of propositional functions serves as a paradigm, what is needed in this regard is an analysis of propositions of the form ' $f(\varphi \hat{x})$ ', or even just of propositions of the form ' $f$ ! $(\varphi ! \hat{x})$ '; for, as the above paradigm indicates, once such an analysis is provided, then quantification with respect to propositional functions is easily explained in terms of contextual definitions similar to the ones given above for classes.

Needless to say, but Russell nowhere explains nor indicates how such an analysis might proceed; and it is unlikely, moreover, that he could provide such an analysis within the primitive framework of the [1908] theory. In particular, just as some form of material equivalence, whether defined or taken primitively, is a necessary component in the above analysis of classes in terms of propositional functions-i.e., necessary for expressing when two propositional functions have the same extension-so too, some form of intensional equivalence (or identity, but as a propositional connective) would be a necessary component in a similar analysis of propositional functions in terms of individuals and propositions of different orders. That is, just as the material equivalence of propositional functions serves to indicate when these functions have the same extension, so too we would need some form of intensional (or necessary) equivalence (as a propositional connective) in order to indicate when, e.g., two proposition/individual pairs have the same intension. But then, even if some such form of intensional equivalence were to be added to the [1908] theory, it would still be unclear how a general analysis of statements about propositional functions, i.e., of statements purporting to have propositional functions as single logical subjects, might proceed; or even whether such an analysis might yet be possible. ${ }^{19}$

\section{Russell's 1910 Multiple Relations Theory of Judgment}

The question of a contextual analysis of statements that purport to have propositional functions as single logical subjects becomes moot in the first edition of $[P M]$, since now, apparently, it is not propositional functions that are nonentities but propositions instead. That is, by a curious turn of fate, propositions are no longer to be considered as single entities, and propositional functions (of different orders) are no longer to be considered as nonentities. Thus, according to Russell, "what we call 'a proposition' (in the sense in which this is distinguished from the phrase express- 
ing it) is not a single entity at all" ([PM], 44), and in fact "the phrase which expresses a proposition is what we call an 'incomplete' symbol" (ibid.).

In denying that a proposition is a single entity, what Russell means to claim is that "a statement in which a proposition appears as subject will only be significant if it can be reduced to a statement about the terms which appear in the proposition" (ibid., 48). In this regard, it should be noted, it is not the assertoric occurrence of a propositional phrase that is an "incomplete symbol," but only the occurrence of a nominalized propositional phrase, i.e., one occurring as a grammatical subject (or object) of a declarative sentence, and especially one occurring as the nominal subject (or direct object) of a sentence expressing a propositional attitude.

We should be cautious in this last regard, however, in distinguishing nominalized propositional phrases that denote events or certain particulars Russell called complexes from those that purport to denote a proposition. For even without propositions, the universe, according to Russell, still "consists of objects having qualities and standing in various relations" (ibid., 43); and some of these objects are complexes denoted by nominalizations of the form 'the-F-ness-of-this', ' $a$-in-the-relation- $\boldsymbol{R}$-to$b$ ', etc. (ibid., 44). A complex, moreover, is not a proposition, but is something "which occurs in the universe and is not simple" (ibid.).

A complex exists, it should be noted, where "a certain number of different entities are combined into a single entity by means of a relation" ([1911], 108); that is, a complex is an instance of a relation in intension, whereas a simple object is an instance of a property (and in particular of a sensible quality). These properties and relations in intension do not exist only in their instances, however, but rather subsist separately in a timeless, platonic realm (cf. [1911], 111). Moreover, it is with just such "universals," as Russell also calls them, that propositional functions are now to be identified.

Such an identification, incidentally, was already made by Russell in [1907] where the following two "principles" were said to be "indispensable if we are both to avoid contradictions and to preserve ordinary mathematics" ([1907], 281):

Any propositional function of $x$ is equivalent to one assigning a property to $x$. 
Any propositional function of $x$ and $y$ is equivalent to one asserting a relation between $x$ and $y$.

These "principles," to be sure, are said in [1907] to be "less evident" than the others that Russell lists there for mathematical logic-which perhaps explains why Russell attempted, albeit unsuccessfully, to do without them in [1908]. And though they are not stated peremptorily in [PM], they are in any case clearly assumed there (as, e.g., in the discussion of the axiom of reducibility on pp. 55-57 and p. 166).

There are difficulties, we agree, in sustaining this interpretation with respect to certain passages of Russell's introduction to the first edition of $[P M]$ (as, e.g., in his discussion of the nature of propositional functions in section 2 of chapter 2). It is clear, however, that in those passages Russell is reiterating his earlier view according to which a propositional function is not determinate prior to, or independently of, the totality of propositions (of some order) that are its values; the view, that is, that assumes that propositions are single entities (of different orders) after all. What is particularly surprising about this apparent relapse to his earlier view, moreover, is that it is immediately followed (in section 3 of chapter 2) by a brief description of Russell's new multiple relations theory of judgment according to which propositions are "false abstractions" and are not to be taken as single entities.

On this theory, according to Russell, "a judgment does not have a single object, namely the proposition, but has several interrelated objects. That is to say, the relation which constitutes judgment is not a relation of two terms, namely the judging mind and the proposition, but is a relation of several terms, namely the mind and what are called the constituents of the proposition" ([PM], 43). Thus, for example, "when we judge (say) 'this is red', what occurs is a relation of three terms, the mind, and [what is denoted by] 'this', and red" (ibid.). Judging is to be distinguished in this regard from perceiving, since "when we perceive [what is denoted by] 'the redness of this', there is a relation of two terms, namely the mind and the complex object [that is denoted by] 'the redness of this' " (ibid.).

The judgment that this is red, as made by some person (say) $A$, is to be represented, accordingly, by a three-place relation combining $A$, this, and red; that is, the judgment is to be analyzed as having the form ' $J_{3}(A$, red, this)' or equivalently, ' $J_{3}(A, \hat{x}$ is red, this)'. Similarly, for an elementary 
statement of the form ' $R_{n}\left(a_{1}, \ldots, a_{n}\right)$ ' the judgment by $A$ that $R_{n}\left(a_{1}, \ldots\right.$, $\left.a_{n}\right)$ is to be analyzed as having the form ' $\mathrm{J}_{n+2}\left(A, R_{n}\left(\hat{x}_{1}, \ldots, \hat{x}_{n}\right), a_{1}, \ldots, a_{n}\right)$ '. A judgment that would ordinarily be taken as having an elementary proposition as its object is now said to be true or false relative not to the truth or falsehood of the elementary proposition in question but to whether there exists a corresponding complex of the objects of the judgment. The judgment is said to be false, in particular, "in the absence of a corresponding complex composed of the objects alone" (ibid., 44), and not because it has an objective falsehood as its object.

A judgment that would ordinarily be taken as having a proposition of the form ' $(x) . \varphi x$ ' as its object, on the other hand, is "a radically new kind of judgment" (ibid., 45); and "the sense in which this judgment is or may be true is not the same as that in which $\varphi x$ is or may be true" (ibid., 46). For, as noted, "if $\varphi x$ is an elementary judgment, it is true when it points to a corresponding complex. But $(x) . \varphi x$ does not point to a single corresponding complex: the corresponding complexes are as numerous as the possible values of $x$ " (ibid.). In this regard, whereas the truth or falsehood of an elementary judgment may be said to be an elementary truth or falsehood, the truth or falsehood of a judgment of this radically new kind is said by Russell to be a second-order truth or falsehood; and similarly we may go on to speak of judgments, or statements in the sense of potential judgments, that have a third-order, fourth-order, etc., truth or falsehood.

Thus instead of taking propositions of different orders as being single entities that are themselves objectively true or false, Russell now assumes that judgments as particular occurrences, or statements as potential judgments, are the primary vehicles of a hierarchically ordered system of truth and falsehood. As a particular occurrence, moreover, a judgment is a complex of entities consisting of a mind and the objects, including the propositional functions (now construed as properties and relations in intension) that would otherwise be taken as the constituents of the proposition being judged. The order of the judgment's truth or falsehood is then to be identified with the maximum order of the propositional functions occurring in that judgment.

As an example of a judgment having a second-order truth or falsehood, consider the judgment that all men are mortal, as made by some person (say) $A$. The analysis of this judgment, on the theory in question, is then to be given as follows: 


$$
J_{4}(A,(x)[\hat{\varphi} ! x \supset \hat{\psi} ! x], \hat{x} \text { is a man, } \hat{x} \text { is mortal }),
$$

where ' $(x)[\hat{\varphi} ! x \supset \hat{\psi} ! x]$ ' represents the second-order relation of material implication between two predicative propositional functions of individuals. ${ }^{20}$ Since the order of this propositional function is the maximum of the orders of the propositional functions occurring in the judgment, then, as defined above, the truth or falsehood of the judgment itself is said to be of the second order.

Similarly, in regard to third-order truth or falsehood, we might consider the judgment made by $A$ that Napoleon had all the predicative properties of a great general. Assuming that ' $f !(\varphi ! \hat{x})$ ' represents a second-order analysis of ' $\varphi \mid \hat{x}$ is a predicative property required in a great general' (cf. $[P M], 56)$, the analysis of the judgment in question would then have the following form:

$$
J_{4}^{\prime}(A,(\varphi)[\hat{\chi} !(\varphi ! \hat{z}) \supset \varphi ! \hat{x}], f !(\hat{\varphi} ! \hat{z}), \text { Napoleon }),
$$

where ' $(\varphi)[\hat{\chi} !(\varphi ! \hat{z}) \supset \varphi ! \hat{x}]$ ' represents a third-order relation between second-order properties of predicative properties of individuals on the one hand and individuals on the other. ${ }^{21}$ Accordingly, since the order of this propositional function is the maximum of the orders of the propositional functions occurring in the judgment, the truth or falsehood of the judgment itself is also of the third order.

\section{The Theory of Ramified Logical Types}

Now if propositional functions are really to be taken as single entities while propositions themselves are no longer to be taken as such, then the order of a propositional function must be given independently of any question regarding the order or existence of propositions as single entities that would otherwise be said to be its values. In this regard, Russell himself restates the presuppositional dependence of a propositional function upon the totality of its values as a dependence instead upon the totality of its arguments (ibid., 54); and he proceeds on that basis to give two informal characterizations of the (ramified) hierarchy of propositional functions (one in section 5 of chapter 2, and the other in "12). Because they are informal, however, the extent to which these characterizations determine exactly the same hierarchy remains unclear, though aside from the 
definitions of predicativity given in each, there seems to be little or nothing to mark an essential divergence of the one from the other.

Matrices in both descriptions, for example, are no longer merely incomplete symbols, but are now described as propositional functions that do not involve any apparent variables. Those that have only individuals for their arguments are said to be first-order matrices; and these together with all propositional functions obtained from them by the process of generalization form the totality of first-order propositional functions. Matrices that have at least one first-order matrix among their arguments but that have no arguments other than first-order matrices and individuals are then said to be second-order matrices; and these together with all propositional functions obtained from them by the process of generalization form the totality of second-order propositional functions. Propositional functions of yet higher orders are then obtained in a completely analogous manner. Thus, "if the highest order of variable occurring in a function, whether as argument or as apparent variable, is a function of the $n$th order, then the function in which it occurs is of the $n+1$ th order." (ibid., 53.) A propositional function is then said to be predicative (in Russell's first characterization) if it is of order $n+1$, where $n$ is the highest order of the arguments of that function, i.e. if it contains no apparent variable of an order higher than $n$. (Only matrices are predicative in Russell's second, and less appropriate, characterization.)

It is preferable of course that we avoid the essential reference to the notion of an apparent variable that occurs in Russell's informal characterization of the order of a propositional function, especially since as single logical subjects (of a given logical type) propositional functions are not to be identified with the linguistic expressions used to represent them. Thus, for example, even though the logical type of a propositional function depends on the logical types of its arguments, it is not uniquely determined by those logical types; that is, "the order of a function may be of any order superior to the order or orders of its arguments" (ibid., 164), and this is so because the order of a propositional function also depends on the order of the apparent variables occurring (in a nonsyntactical sense) in that propositional function. It is with respect to this additional dependence, incidentally, that two propositional functions may have arguments of the same logical type(s) - and, in that regard, they may even determine the same extension (as is assumed in the axiom of reducibility)-without 
themselves being functions of the same order or logical type; and it is with respect to the possible differences in orders of the propositional functions having arguments of the same logical type(s), moreover, that Russell's theory of logical types is said to be ramified.

Fortunately, Alonzo Church has provided us with both a formal and independent (recursive) characterization of Russell's ramified logical types, hereafter called $r$-types, and of their orders; that is, a characterization that is not only formal rather than informal but that is also independent of the notion of the occurrence (in a non-syntactical sense) of an apparent variable in a propositional function (cf. Church, "Comparison of Russell's resolution of the semantical antimonies with that of Tarski," ([2]), 748):

there is an r-type $i$ to which all and only individuals belong, and whose order is stipulated to be 0 .

If $m \in \omega, n \in \omega-\{0\}$, and $\beta_{1}, \ldots, \beta_{m}$ are any given $\mathrm{r}$ types, then there is an $r$-type $\left(\beta_{1}, \ldots, \beta_{m}\right) / n$ to which belong all and only $m$-ary propositional functions of level $n$ and with arguments of $r$-types $\beta_{1}, \ldots, \beta_{m}$, respectively; and the order of such a function is $\mathrm{N}+n$, where $\mathrm{N}$ is the greatest of the orders corresponding to the types $\beta_{1}, \ldots, \beta_{m}$ (and $\mathrm{N}=0$ if $m=0$ ).

The notion of the level of a propositional function of $\mathrm{r}$-type $\left(\beta_{1}, \ldots, \beta_{m}\right) /$ $n$ is required in this characterization, it should be noted, as a counterpart to Russell's use of the notion of an apparent variable occurring in that propositional function. That is, with respect to both Russell's informal and Church's formal characterization, if $\mathrm{N}$ is the greatest of the orders corresponding to $\beta_{1}, \ldots, \beta_{m}$, and $k$ is the greatest of the orders of the apparent variables occurring (in Russell's implicit non-syntactical sense) in that function, then $n=1$ if $k \leq \mathrm{N}$, and $n=k+1$ if $\mathrm{N}<k$. Thus, for example, a propositional function of $\mathrm{r}$-type $\left(\beta_{1}, \ldots, \beta_{m}\right) / n$ is a predicative function iff $n=1$; and in that regard the axiom (schema) of reducibility may be therefore stated as follows:

$$
(\varphi)(\exists \psi)\left(x_{1}\right) \ldots\left(x_{m}\right)\left[\varphi\left(x_{1}, \ldots, x_{m}\right) \equiv \psi\left(x_{1}, \ldots, x_{m}\right)\right],
$$


where ' $\varphi$ ', ' $\psi$ ' are variables (for propositional functions) of r-types $\left(\beta_{1}, \ldots\right.$ ,$\left.\beta_{m}\right) / n$ and $\left(\beta_{1}, \ldots, \beta_{m}\right) / 1$, respectively, for some $n \in \omega-\{0\}$, and ' $x_{1}$ ', ' $x_{2}$ ', ., ' $x_{m}$ ' are variables of $r$-types $\beta_{1}, \ldots, \beta_{m}$, respectively. (The characterization of $r$-types and orders is of course intended to apply syntactically to variables and other expressions as well as to the individuals and propositional functions that are purportedly the values of those variables.)

Similarly, although Russell did not himself explicitly formulate any comprehension principles in $[P M]$, it is clear by his cap-notation for designating propositional functions that the following comprehension principle, as stated with respect to Church's characterization, is intended to be taken as valid:

$$
(\exists \psi)\left(x_{1}\right) \ldots\left(x_{m}\right)\left[\psi\left(x_{1}, \ldots, x_{m}\right) \equiv A\right],
$$

where ' $\psi$ ' is a variable of $r$-type $\left(\beta_{1}, \ldots, \beta_{m}\right) / n, x_{1}{ }^{\prime}, \ldots, x_{m}$ ' are distinct variables of $\mathrm{r}$-types $\beta_{1}, \ldots, \beta_{m}$, respectively, and the bound (apparent) variables of the formula $A$ are all of orders less than the order of ' $\psi$ ', and the free (real) variables and constants occurring in $A$ are all of orders not greater than the order of ' $\psi$ '.

In regard, finally, to the status of propositions in the ramified theory of logical types, it should be noted that both Russell and Church allow for their inclusion, but not in such a way that the hierarchy of propositional functions would not make sense without them. For Russell in particular, "the propositional hierarchy can ... be derived from the functional hierarchy, and we may define a proposition of the $n$th order as one which involves apparent variables of the $n-1$ th order in the functional hierarchy" (ibid., 55). With respect to Church's characterization, on the other hand, propositions of order $n$ are counted as 0-ary propositional functions of level $n$, i.e., as propositional functions of r-type ( )/n, where '( )' represents the null-sequence.

There is no essential need for taking propositions as single entities in either characterization, however; or at least there is no such need on the assumption that Russell's multiple relations theory of judgment is adequate for the analysis of statements expressing propositional attitudes, i.e. on the assumption that statements that purport to have propositions as single logical subjects can in general be analyzed in terms of the objects, including the propositional functions, that would otherwise be said to oc- 
cur in those propositions. We can easily modify Church's characterization of $r$-types to reflect this position, moreover, by simply excluding all r-types of the form $\left(\beta_{1}, \ldots, \beta_{m}\right) / n$ where $m=0$, i.e. by requiring in clause (2) above of the definition of r-type that $m \in \omega-\{0\}$.

It is noteworthy, incidentally, that Church does not believe that Russell's framework is coherent without propositions as single entities; but this is because he himself rejects the multiple relations theory of judgment. In particular, Church maintains, the "fragmenting of propositions" that is involved in the multiple relations theory of judgment also requires the "fragmenting of propositional functions" (cf. [2], 748). This is not actually so, however, as the examples of section 9 indicate; for whereas the propositional functions that occur in a judgment are indeed "fragmented" in the sense of analysis, nevertheless each propositional function, as well as the "fragments" of that function that result upon analysis, retains its status in the judgment as a single entity. Indeed, without including propositional functions among the single entities that are combined in the judgment complex, there would be no multiple relations theory of judgment at all. The "fragmenting" of the proposition supposedly being judged, on the other hand, is designed with the specific result of eliminating the proposition as a single entity or logical subject of that judgment.

Notwithstanding the exclusion on this analysis of propositions as single entities, we need not also exclude Russell's hierarchy of statements (in the sense of potential judgments), i.e. we need not exclude "propositions" that occur, (and can occur) only assertorically; and this in fact seems to be all Russell now really intends in speaking of the hierarchy of propositions. What this would amount to with respect to Church's characterization of r-types is that whereas there are 0-ary propositional functions of each level $n$, nevertheless none of these "propositions" can themselves occur as arguments or logical subjects of other propositional functions, i.e. that none of the r-types $\beta_{1}, \ldots, \beta_{m}$ of an r-type of the form $\left(\beta_{1}, \ldots, \beta_{m}\right) / n$, where $m>0$, are themselves of the form ( ) $/ k$, for some positive integer $k$.

\section{Concluding Remarks on Russell's 1925 Introduction to Principia Mathematica}

As we said in our introductory remarks, our concern in this chapter is with the development of the theory of logical types and of the notion of a 
logical subject in Russell's early philosophy, i.e., in the views developed by Russell up to, but not beyond, the 1910-13 publication of the first edition of $[P M]$. Nevertheless, having noted and examined one curious turn of fate in the development of Russell's views regarding the respective logical roles of propositions and propositional functions, it is perhaps appropriate that we at least note and briefly comment on what appears to be the opposite turn of fate in the development of Russell's views from 1913-25, i.e. from 1913 until Russell's 1925 introduction (and added appendices) to the second edition of $[P M]$. In particular, in this regard, it should be noted (1) that propositions, and especially atomic propositions-since apparently all others are now to be taken as (possibly infinite) truth-functions of atomic propositions-are once again to be taken in a fundamental way in Russell's implicit semantical framework; and (2) that no propositional function can occur in a proposition other than through its values, i.e., that no propositional function can occur as a logical subject, or, equivalently, that a propositional function can only have an assertoric occurrence in a proposition.

In regard first to the latter point, it should be noted that sometime in 1913, after extensive discussion with Wittgenstein, Russell came to believe that a property or relation in intension could no longer occur significantly as a term, i.e. as a single logical subject, and that therefore the multiple relations theory of judgment was to be rejected (cf. Russell, My Philosophical Development, ([MPD]), 182). A predicate, Russell says in [1918], "can never occur except as a predicate" (Logic and Knowledge, ([L\&K]), 205); and "a relation can never occur except as a relation, never as a subject" (ibid., 206). Apparently, it is with this sort of view in mind that Russell is led to claim in his 1925 introduction to $[P M]$ that "there is no logical matrix of the form $f !(\varphi ! \hat{z})$ " (p.xxxi). With such a change in both his semantics and his syntax, however, it is no longer clear what is left of the theory of logical types (beyond r-types of the form $(i, \ldots, i) / n)$, or how, in particular, Russell can continue to carry through his program for a higher order logical analysis of mathematical statements. These are issues that we shall take up and answer in chapter 5 .

Secondly, although propositions are reintroduced into Russell's implicit semantical framework in a fundamental way, nevertheless they are still not to be taken as single logical subjects, and in particular they still cannot occur as logical subjects (i.e., as direct objects) of propositional at- 
titudes (cf. $[P M]$, second edition, Appendix C). A statement of the form 'A believes $p$ ', in other words, "is not a function of $p$ " (ibid., xiv). In this regard, according to Russell, we should "distinguish between a proposition as a fact and a proposition as a vehicle of truth or falsehood" ([PM], Appendix C, p. 402); for "it is the proposition considered factually that occurs in such statements as ' $A$ believes $p$ ' and ' $p$ is about $A$ ' " (ibid., 407).

Considered factually, however, a proposition is not the same as an objective truth or falsehood in the sense of Russell's earlier [1908] theory, but is instead the content of a propositional attitude (cf. [1919], 308). As such a content, moreover, a proposition has an "objective reference," viz. a fact, and it is that fact that makes the proposition true or false. Nevertheless, "propositions are not names for facts" ([1918], 187); for, according to Russell, "you cannot properly name a fact. The only thing you can do is to assert it, or deny it, or will it, or wish it, or question it," etc., (ibid., 188). That is, "you can never put the sort of thing that makes a proposition to be true or false in the position of a logical subject" (ibid.).

Now if a fact cannot be a logical subject, then neither can a proposition, since a proposition is itself a fact "having a certain analogy of structure ... with the fact which makes it true or false" ([1919], 309). "Propositions are facts," (ibid., 315); and therefore no proposition is the sort of thing that can occur in another proposition "in the position of a logical subject." In this regard, accordingly, Russell cannot have the implicit semantical framework of the 1908 theory of types in mind in his 1925 reintroduction of propositions as entities to which propositional functions are to be reduced.

Needless to say, but as these comments indicate, it is doubtful whether the implicit semantical framework Russell seems to have in mind in 1925 is really appropriate for the theory of (higher order) logical types after all. This is not to say of course that Russell's later philosophical views are of lesser importance than his earlier, but only that they are views that do not accord with what today would be called a predicate logic of third or higher order, i.e. with a logic in which propositional functions, and perhaps propositions as well, can occur as logical subjects. 


\section{Notes}

1 Though it is only classes as many that are ultimately involved in the analyses in [POM] of traditional mathematical propositions, certain classes as one of individuals are taken by Russell in Appendix B to be "the objects of daily life" ([POM], 523). For example, "a person is a class [as one] of psychical existents," and tables, chairs, apples, etc., are classes as one of material points.

2 For reasons indicated in section 5 below, it would suit Russell's purposes better if he took the adjectival concept to be derived from the common noun concept by means of ellipsis. A predicate, on this interpretation, is then an elliptically expressed class-concept corresponding to the adjectival modification of a common noun.

3 This terminology is not used by Russell in [POM]; but it is a natural interpretation of his remarks, and it can be compared with the definitions of predicativity he gives in [1908] and in [PM]. Indeed, the equivalence of this notion of predicativity with the one that he defines in [1905], and that is described below, is in fact the basis of Russell's attempted justification in [1908], and in [PM], of the axiom of reducibility, which says in effect that every propositional function of a given type is equivalent to a predicative propositional function of that type.

4 It is of course problematic regarding how quantification over propositional functions is to be interpreted in $[P O M]$, though at certain passages in $[P O M]$, Russell seems to think that such quantification is in principle eliminable, or at least explainable, somewhat in the manner of the substitutional theory described in section 6 below.

5 Cf. $[P M], 166$.

(6 Russell himself associated the zig-zag theory with the theory of logical types briefly outlined in Appendix B of [POM], a theory that corresponds well with the simple theory of (homogeneous) types. Quine's system NF, originally devised as a first-order counterpart to the (monadic) theory of simple types is occasionally described as a paradigm zig-zag theory. NF, however, exceeds the powers of the theory of simple types in a number of ways, and it is not really a theory in which propositional functions can be considered as single entities (over and above the classes they define). If, on the other hand, instead of using Quine's stratified version of $(\mathrm{CP}-\epsilon)$ in a first-order theory, we turned to a stratified version of $\left(\mathrm{CP}^{*}\right)$ in a second-order logic that allows for nominalized predicate variables, we obtain the system of monadic $\mathrm{ST}^{*}$ described in [3], and which is shown there to be equi-consistent with the monadic theory of simple types. In the relational or non-monadic case, however, $\left(\mathrm{CP}^{*}\right)$ must be homogeneously stratified, on pain otherwise of regenerating Russell's paradox of predication. The resulting HST* (described in section 10 of my "The theory of homogeneous simple types as a second order logic," ([3]) allows nevertheless for an alternative version of Russell's "no classes" theory, since it by-passes ( $C P-\in$ ) altogether. Indeed Russell himself stated that "technically, the theory of types suggested in Appendix B differs little from the no-classes theory" ([1906b], 193).

7. Cf. [1906a], 173, where the concept bumanity is taken as a complex entity whose constituents are other concepts.

8 According to Russell, "it is not necessary that $p$ should be a proposition; if $p$ is any en- 
tity other than a proposition, and $a$ is any entity other than a proposition, and $a$ is any entity other than $p, p / a ; x$ will never be truc, and thus pla is null" (ibid., 175).

9 Cf. [1906b], 203. The proof, as Russell gives it for propositions, may be briefly stated as follows: Beginning with two entities, say, $a$ and $u$, it can be shown that each of the propositions determined by the following recursion are all different:

$$
\begin{aligned}
& \text { (1) } p_{0}=\mathrm{df}(a=u) \\
& \text { (2) } p_{n+1}=\mathrm{df}\left(p_{n}=u\right) .
\end{aligned}
$$

10 Cf. Henri Poincaré, "Les Mathematique et a logicpue." ([6]). Poincaré also claimed, it should be noted, that it was the assumption of an actual (as opposed to the merely potential) infinite that is also responsible for the paradoxes.

11 A proposition in the sense of $[P O M]$ can be said to contain an apparent variable (in a non-syntactical sense) if one of its constituents is a denoting concept containing a propositional function as its class-concept component. The same "explanation" could also be applied to Russell's 1905 theory of denoting-except that now a denoting concept will involve only the operations associated with 'every' and 'some'-but Russell himself, by rejecting denoting complexes altogether (not to mention his rejection of propositional functions), in effect blocks such an "explanation." Of course, since no proposition in the present 1906 theory can contain an apparent variable, then no such explanation is needed. The problem does arise, however, in the [1908] theory of logical types, which may well be one of the reasons for Russell's later rejection of propositions as single entitics in the first edition of [ $P M]$.

12 A statement in this sense is a judgment, or perhaps a potential judgment since Russell does not intend to claim that all statements do in fact occur at some time or other. Given Russell's own reference (p. 207) in [1906b] to his carlier 1904 Meinong review (reprinted in Russell's Essays in Analysis, ([4])), I believe that we can roughly identify Russell's implicit 1906 theory of judgment with the 1904 theory described in his Meinong review-except, that is, for the modifications demanded by his new 1905 theory of denoting phrases. A judgment in this sense is the occurrence of an act together with a content (or state of mind) that has a proposition as an objective truth or falschood as its object. (Cf. [4], 60: "the judgment itself, in its purity, as something wholly psychical, is merely the content and the act: the object is not part of the judgment".) Needless to say, but this modified 1904 theory of judgment is radically different from the multiple relations theory described in [PM], 43-46, and discussed in section 9 below.

13 The type of a statement, and the resulting hierarchy of statements, described here should not be confused with the type (or order) of a proposition in [1908]. In addition, the only type of variable involved in the present theory is the unrestricted variable, whereas in [1908] there are different types of variables for different types of propositions.

14 That is, within the substitutional theory the principle of mathematical induction will have the following form: 


$$
\begin{aligned}
&(p)(a)(p / a ; 0 \&(x)[p / a ; x \supset p / a ;(x+1)] \supset \\
&(x)[x \text { is a finite cardinal number } / x ; x \supset p / a ; x]) .
\end{aligned}
$$

15 Russell explicitly identifies properties with propositional functions of one argument in [1905] and continued to do so through the first edition of [PM]. Relations in intension are not explicitly identified with propositional functions of more than one argument until [1907], however; but the identification, once made, is definitely continued through the first edition of $[P M]$.

16 Russell is not unaware of this limitation, incidentally, as witness his qualification that a statement may be replaced by an equivalent statement only "otherwise than in a matrix" (ibid., 212). Russell, unfortunately, slips into old habits here and uses "proposition' instead of 'statement' in describing this limitation. It is of course only statements, or statement forms, that are interchanged, since the whole point is to replace one that contains an apparent variable with one that does not; and on the substitutional theory it is statements, and not propositions, that can contain an apparent variable.

17 Russell deliberately avoids any discussion in [1908] of the distinction or similarity between statements and propositions, especially as regards the sense in which the latter may also be said to contain an apparent variable. Such a discussion is avoided, apparently, because it would raise philosophical questions that are "essentially separable from the mathematical development of the theory, and, like all philosophical questions, introduce elements of uncertainty which do not belong to the theory itself" ([1908], 102).

It should be noted, nevertheless, that an "explanation" of how a proposition can contain an apparent variable (in a non-syntactic sense) cannot be given in terms of the occurrence in that proposition of a denoting concept consisting of one of the operations associated with 'every' and 'some' as applied to a propositional function; for, according to Russell, "denoting phrases never have any meaning in isolation, but only enter as constituents into the verbal expression of propositions which contain no constituent corresponding to the denoting plorases in question" ([1908), 70, italics added). Quantifier phrases, accordingly, camnot be taken as representing "second-level" concepts (in the Fregean sense) that can occur as constituents of propositions containing an apparent variable (in a non-syntactical sense).

18 As Rudolf Carnap in Meaning and Necessity, ([1], section 3.3) has observed, Russell's particular analysis has certain unnatural consequences. For example, while ' $\hat{z}(1 / z)=\| \hat{z}$ '

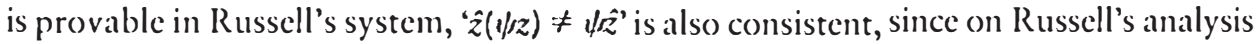
the latter reduces to:

$$
(\exists \varphi)((x)[\varphi ! x \equiv \psi x] \& \varphi ! \hat{z} \neq \neq \psi \hat{z}) .
$$

Carnap's alternative analysis,

$$
f\{\hat{z}(\psi z)\}=\mathrm{d} f(\varphi)((x)[\varphi ! x \equiv \| / x] \supset /\{\varphi ! \hat{z}\}),
$$

avoids these consequences, since, in particular, ' $\hat{z}(\psi / z)=\| / \hat{z}$ ' is no longer provable. (A similar alternative analysis is also to be preferred-for some, even if not for all, linguistic contexts-to Russell's contextual analysis of definite descriptions.) 
19 It should be noted in this regard that an analysis of the form:

$$
f\{\varphi \hat{x}\}=\mathrm{d} f(p)(a)((x)[p / a ; x \equiv \varphi x] \supset f\{p / a\}),
$$

where '포' represents the intensional (or necessary) equivalence in question, will not do, since the expression ' $f p / a\}$ ' is meaningless in the [1908] framework. ( $p / a$ ' is, after all, an incomplete symbol.) Similarly, an analysis of the form:

$$
f\{\varphi \hat{x}\}=\mathrm{d} f(p)(a)((x)[p / a ; x \equiv \varphi x] \supset\{\{p\})
$$

will not do since (1) properties of propositional functions are not in general the same as properties of propositions, and (2) the analysans is equivalent to:

$$
(p)((\exists a)(x)[p / a ; x \equiv \varphi x] \supset\{\{p\}),
$$

which can lead to both ' $f \varphi \hat{x}\}$ ' and ' $f\{\varphi \hat{x}\}$ ' depending on to which of several terms occuring in a proposition the existential quantifier is intended to apply.

20 In terms of the definition of $r$-type and order given below in section 10, and on the assumption that ' $\varphi \mid \hat{x}$ ' and ' $\psi \mid \hat{x}^{\prime}$ ' are of r-type (i)/1, and therefore of order 1 , the expression ' $(x)[\hat{\varphi} ! x \supset \hat{\psi} ! x]$ ' is easily seen to be of r-type $((i) / 1,(i) / 1) / 1$, and therefore, by definition, of order 2.

21 Both ' $f !(\hat{\varphi} ! \hat{z})$ ' and ' $\chi$ ! $(\hat{\varphi} ! \hat{z})$ ', by assumption, are of r-type $(i / 1) / 1$, and therefore of order 2. The expression

$$
(\varphi)[\hat{\chi} !(\varphi ! \hat{z}) \supset \varphi ! \hat{x}],
$$

one of whose instances is

$$
(\varphi)[f !(\varphi ! \hat{z}) \supset \varphi ! \text { Napoleon }],
$$

has arguments of r-type $(i / 1) / 1$ and $i$, respectively; and therefore it is of r-type $((i / l) / 1, i) /$ 1 and of order 3 .

' $J_{4}$ ' and ' $J_{4}^{\prime}$ ' do not represent the same four-place judgment relation, incidentally, since their r-types (and orders) are clearly different.

\section{References}

[1] Carnap, Rudolf, Meaning and Necessity. Chicago: University of Chicago Press, 1956.

[2] Church, Alonzo. "Comparison of Russell's resolution of the semantical antinomies with that of Tarski," The Journal of Symbolic Logic 41 (1976), 747-60.

[3] Cocchiarella, Nino. "The theory of homogeneous simple types as a second order logic," Notre Dame Journal of Formal Logic 20 (1979), 505-24; and "Two $\lambda$ Extensions of the Theory of Homogeneous Simple Types as a Second-Order Logic," Notre Dame Journal of Formal Logic 26 (1985), 377-407.

[4] Lackey, Douglas, editor, Essays in Analysis by Bertrand Russell. New York: Braziller, 1973.

[L\&K] Marsh, Robert, editor, Logic and Knowledge, London: Allen and Unwin, 1956. 
[6] Poincarć, Henri, "Les mathematiques et la logique," Revue de Metaphysique et du Morale 14 (1906), 17-34.

[POM] Russell, Bertrand, The Principles of Mathematics, 2nd edition with new intro., N.Y.: Norton \& Co., (1938).

[1905] Russell, Bertrand, "On some difficulties in the theory of transfinite numbers and order types." Proc. of the London Math. Soc. (March 1906). (First read publicly in 1905, and reprinted in [4].)

[1906a] Russell, Bertrand, "On the substitutional theory of classes and relations," read before the London Math Society in May 1906, but first published only in [4].

[1906b] Russell, Bertrand. "On 'Insolubilia' and their solution by symbolic logic," first published as "Les paradoxes de la logique," in Revue de Metaphysique et de Moral 14 (1906), 627-50. The English translation appears in [4].

[1907] Russell, Bertrand, "The regressive method of discovering the premises of mathematics," read before the Cambridge Math. Club in March 1907 and first published in [4].

[1908] Russell, Bertrand, "Mathematical logic as based on the theory of types," American Journal of Mathematics 30 (1908), 222-62. Reprinted in [5].

[PM] Russell, Bertrand, and Whitehead, A.N., Principia Mathematica, vol. 1 (1910), vol. 2 (1912) and vol. 3 (1913), London: Cambridge University Press. Partially reprinted as Principia Mathematica to *56, with Russell's 1925 intro. to the second edition and his 1925 appendices A and C, Cambridge University Press, 1962. (Pagination is made to this 1962 edition.)

[1911] Russell, Bertrand. "On the relations of universals and particulars," Proc. of the Aristotelian Society (1911-12). Reprinted in [5].

[1918] Russell, Bertrand, "The philosophy of logical atomism," The Monist 28 (1918), 495-527. Reprinted in [5].

[1919] Russell, Bertrand, "On propositions: What they are and how they mean," Proc. of the Aristotelian Society, supp. vol. 2 (1919), 1-43. Reprinted in [5].

[MPD] Russcll, Bertrand, My Philosophical Development, London: Allen and Unwin, 1959. 


\section{Frege, Russell, and Logicism: A Logical Reconstruction}

Logicism by the end of the nineteenth century was a philosophical doctrine whose time had come, and it is Gottlob Frege to whom we owe its arrival. "Often," Frege once wrote, "it is only after immense intellectual effort, which may have continued over centuries, that humanity at last succeeds in achieving knowledge of a concept in its pure form, in stripping off the irrelevant accretions which veil it from the eyes of the ? mind" (Frege, The Foundations of Arithmetic, $[F d]$, xix). Prior to Frege logicism was just such a concept whose pure form was obscured by irrelevant accretions; and in his life's work it was Frege who first presented this concept to humanity in its pure form and developed it as a doctrine of the first rank.

That form, unfortunately, has become obscured once again. For today, as we approach the end of the twentieth century, logicism, as a philosophical doctrine, is said to be dead, and even worse, to be impossible. Frege's logicism, or the specific presentation he gave of it in Die Grundgesetze der Arithmetik, ([Gg]), fell to Russell's paradox, and, we are told, it cannot be resurrected. Russell's own subsequent form of logicism presented in $[P M]$, moreover, in effect gives up the doctrine; for in overcoming his paradox, Russell was unable to reduce classical mathematics to logic without making at least two assumptions that are not logically true; namely, his assumption of the axiom of reducibility and his assumption of an ax- 
iom of infinity regarding the existence of infinitely many concrete or nonabstract individuals.

Contrary to popular opinion, however, logicism is not dead beyond redemption; that is, if logicism is dead, then it can be easily resurrected. This is not to say that as philosophical doctrines go logicism is true, but only that it can be logically reconstructed and defended or advocated in essentially the same philosophical context in which it was originally formulated. This is true especially of Frege's form of logicism, as we shall see, and in fact, by turning to his correspondence with Russell and his discussion of Russell's paradox, we are able to formulate not only one but two alternative reconstructions of his form of logicism, both of which are consistent (relative to weak Zermelo set theory).

In regard to Russell's form of logicism, on the other hand, our resurrection will not apply directly to the form he adopted in $[P M]$ but rather to the form he was implicitly advocating in his correspondence with Frege shortly after the completion of $[P O M]$. In this regard, though we shall have occasion to refer to certain features of his later form of logicism, especially in our concluding section where a counterpart to the axiom of reducibility comes into the picture, it is Russell's early form of logicism that we shall reconstruct and be concerned with here.

Though Frege's and Russell's early form of logicism are not the same, incidentally, they are closely related; and one of our goals will be to reconstruct or resurrect these forms with their similarity in mind. In particular, it is our contention that both are to be reconstructed as second order predicate logics in which nominalized predicates are allowed to occur as abstract singular terms. Their important differences, as we shall see, will then consist in the sort of object each takes nominalized predicates to denote and in whether the theory of predication upon which the laws of logic are to be based is to be extensional or intensional.

\section{Logicism and the Predicative Nature of Concepts}

The doctrine of logicism can be succinctly stated in the following two- 2 fold claim: (1) that all of the concepts of classical mathematics are explicitly definable in terms of purely logical concepts; and (2) that all of the theorems of classical mathematics can be derived from the laws of logic through purely logical deductions (cf. [Carnap]). This is not a doctrine 
about the reducibility of mathematics to set theory, it should be noted, but about the reducibility of classical mathematics to the concepts and laws of logic. In other words, it is not a doctrine about the reducibility of mathematics to a theory of membership (in a class or set) but about the reducibility of mathematics to a theory of predication, and in particular to a theory about the concepts that predicates stand for in their role as predicates. For this reason both Frege and Russell maintained that the logistic framework within which classical mathematics is to be represented must consist at least of a second order predicate logic where quantification is not only with respect to the role of singular terms but to that of predicates as well. Indeed, it was Frege himself who first formulated and developed standard second order predicate logic, and he did so precisely as a framework within which classical mathematics was to be reduced to logic.

The distinction between predicates and singular terms, it must be emphasized, is fundamental to both Frege's and Russell's forms of logicism; and to fail to attend to this distinction is to fail to understand the nature of predication in either framework. In particular, the attempt to characterize predication in terms of a first order theory of exemplification (or a first order theory of membership without an axiom of extensionality) is a nonstarter as far as either form of logicism is concerned. Predicates are not singular terms or what Frege called "proper names" in his extended sense, and the role of concepts in predication is not that of objects or individuals to which other objects or individuals stand in a relation of exemplification.

Now this does not mean that predicates cannot be nominalized and transformed into singular terms the way that 'human', 'triangular', 'wise', etc., can be transformed into 'humanity', 'triangularity', 'wisdom', etc.; or to use Frege's example, the way 'horse' can be transformed into 'the concept horse'. Rather, the point is that any account of nominalized predicates as abstract singular terms presupposes an account of their role as predicates; and in particular any relational predicate such as 'exemplifies' or 'falls under', as in 'Socrates exemplifies humanity' and 'Bucephalus falls under the concept horse', is to be viewed as derived from an account of predication in which predicates do not have such

$\Delta \Delta$ nominalized forms. Indeed, the priority of the role of a predicate as a predicate over the corresponding role of its nominalization as an abstract singular term is in fact one of the ways we are to understand Frege's famous context principle (cf. $[F d]$, xxii). For it is only in the context of a sen- 
tence that a predicate can occur as a predicate, and it is only through a correlation with such occurrences that we are to understand the role of a nominalized predicate as an abstract singular term... This sort of correlation is fundamental not only to Frege's logicism but also to the reconstruction we shall propose.

What distinguishes a predicate from its nominalization, according to Frege, is that a predicate has an unsaturated nature that is essential to its role in predication. That is, whereas a nominalized predicate is a com-. plete, saturated expression in its own right, the predicate itself is in need of supplementation, and it is this unsaturatedness or need of supplementation that is the basis of its predicative nature. Moreover, because this unsaturatedness is essential to its role in predication, the concept that a predicate stands for (bedeutet) is said by Frege to have a corresponding unsaturated nature as well. Indeed, it is precisely the corresponding unsaturatedness of a concept that Frege identifies as its predicative nature (cf. Translations from the Philosophical Writings of Gottlob Frege, ([G\&B]), 47; and Frege's Posthumous Writings, ([PW]), 177).

Now just as the predicative nature of a predicate excludes its being a singular term, so too, according to Frege, the predicative or unsaturated nature of a concept excludes it from being the denotatum (Bedeutung) of a singular term; and therefore what a nominalized predicate denotes, according to Frege, is not the concept that the predicate otherwise stands for in its role as a predicate. Instead, according to Frege, the concept "must first be converted into an object, or, speaking more precisely, represented by an object" ([G\&B], 46), and it will be this object, or conceptcorrelate as Frege also calls it, that is the real denotatum of the nominalized predicate. Thus, in particular, what 'the concept horse' denotes, according to Frege, is not a concept but an object, i.e., a conceptcorrelate.

This is not the conclusion Russell came to in $[P O M]$ and thereafter, it should be noted; and in fact, as already indicated, Russell's different view in this regard is one of the principal differences between his and Frege's form of logicism. Russell did hold something rather similar to Frege's view in an unpublished manuscript of 1898; but in [POM], it is clear, he explicitly rejects the earlier view. Thus (keeping in mind that terms and predicates for Russell are individuals and concepts, respectively, and not expressions) in 1898 he wrote that "the peculiarity of predicates is that 
they are meanings," and "although it is impossible to speak of meanings without making them subjects ..., yet meanings as such are the antithesis of subjects, are destitute of being, and incapable of plurality. When I say 'Socrates is human', human as used in this judgment does not have being, and is not a logical subject. I am, in a word, not asserting a relation between two subjects. As soon as I make human a term, . . . I have added something, namely being, one-ness, and diversity of being from other terms which human as predicate did not possess" (Russell, An Analysis of Mathematical Reasoning, ([AMR]), bk. 1, p. 10).

Russell briefly restates this view in $[P O M]$, observing that "it might be thought that a distinction ought to be made between a concept as such and a concept as used as a term" (p. 45); but he quickly rejects it, claiming that "inextricable difficulties will envelop us if we allow such a view" (ibid.) The difficulties in question, it turns out, are those connected with such claims as that the concept horse is not a concept, which Russell thinks is false and leads to a contradiction. His conclusion is that "terms which are concepts differ from those which are not, not in respect of selfsubsistence, but in virtue of the fact that, in certain true or false propositions, they occur in a manner which is different in an indefinable way from the manner in which subjects or terms of relations occur" (ibid., $\triangle 46)$. In other words, concepts, including relations, are individuals (since 'term' for Russell is synonymous with 'individual'-cf. [POM], 43), and they are denoted as such by nominalized predicates occurring as abstract singular terms. Yet, predicate expressions are not singular terms and the concepts that predicate expressions stand for in their role as predicates do not occur in propositions the way that individuals denoted by singular terms do. That is, even though concepts, according to Russell, do not have an unsaturated nature, nevertheless they do have a predicative nature in virtue of which they can occur in propositions in "a manner which is different in an indefinable way" from that in which individuals denoted , by singular terms occur.

Now the "indefinable way" in which a concept can occur in a proposition as a concept and not as a term, according to Russell, is also a feature of what he calls a propositional function; and in fact in [POM] a propositional function can occur in a proposition only in this "indefinable way." That is, unlike concepts', propositional functions in [POM] are not individuals. To be sure, to each concept there corresponds a unique proposi- 
tional function in the propositional values of which the concept occurs as a concept; but upon discovering his paradox, Russell was led in [POM] to doubt that every propositional function is either itself a concept or that it has a concept corresponding to it. Of course, "apart from the contradiction in question," Russell observes, "this point might appear to be merely verbal: 'being an $x$ such that $\phi x$ ', it might be said, may always be taken as a predicate [concept]. But in view of our contradiction," he continues, "all remarks on this subject must be viewed with caution" (ibid., 88). In particular, the contradiction is avoided for Russell in [POM] "by the recognition that the functional part of a propositional function is not an independent entity" (ibid.); and in fact it was precisely for this reason that Russell was led in his commentary on Frege (in Appendix A of [POM]) to claim that "the word Begriff is used by Frege to mean nearly the same thing as propositional function" (p. 507).

In his later form of logicism in $[P M]$, Russell was able to avoid his para- $2 A$ dox while also claiming that propositional functions are single entities after all; i.e., that propositional functions are individuals, albeit of a higher order/type than concrete individuals (a fact that is often missed or ignored by philosophers and logicians alike since, contrary to his earlier practice, Russell chose to use 'individual' in $[P M]$ only to refer to concrete individuals). There is no point in distinguishing concepts from propositional functions in such a framework, needless to say, and in fact we shall not assume any such distinction even in our resurrection of the form of logicism that is implicit in Russell's correspondence with Frege shortly after $[P O M]$ was written. That is, having noted the difference between concepts and propositional functions that Russell thought he was committed to in $[P O M]$ as a result of his paradox, we shall nevertheless assume that concepts, including relations, are none other than propositional functions, since that in fact was what Russell originally thought and returned to in his later form of logicism. We return in this way to the original context of Russell's paradox as it applies to Russell's early form of logicism no less so than as it applies to Frege's form of logicism. In that regard, in $x \times$ other words, we may assume that the only important difference between Frege's and Russell's early form of logicism that need concern us at this point is that whereas according to Frege nominalized predicates denote concept-correlates, i.e., objects somehow correlated with concepts, for Russell nominalized predicates denote the same concepts or propositional 
functions that the predicates in question otherwise stand for in their role as predicates. What is common to both forms of logicism, on the other hand, is that the concepts assumed in each form have a predicative nature, and that it is this predicative nature that is the basis of the laws of. logic.

\section{Predication versus Functionality}

Standard second order predicate logic with identity, we have already noted, was first formulated by Frege as a framework in which to carry out the reduction of classical mathematics to logic. The reduction is not forthcoming, to be sure, without a logistic treatment of nominalized predicates or some such equivalent device, but still it is a framework in which the laws of logic have their basic form prior to any assumption about how nominalized predicates are to be interpreted. In this regard it is a system of the laws of logic that are common to both Frege's and Russell's early form of logicism; and for that reason we shall turn to its formulation first.

Accordingly, let us use ' $x$ ', ' $y$ ', and ' $z$ ', with or without numerical subscripts, to refer (in the metalanguage) to individual variables, and similarly let us use ' $F$ ', ' $G$ ', and ' $R$ ' to refer to n-place predicate variables. (We shall usually delete the superscript when the context makes clear the number of subject or argument positions that go with a predicate variable or constant; and we shall use ' $R^{\mathrm{n}}$ ' only when $\mathrm{n}>1$.) As primitive logical constants, let us take $\rightarrow$ (the material conditional sign), $\sim$ (the negation sign), = (the identity sign), and $\forall$ (the universal quantifier sign). As usual, we understand the juxtaposition of signs to represent their concatenation. We shall also use parentheses and brackets as auxiliary signs.

Ignoring the introduction of predicate and individual constants for the special applications of logic, the basic or atomic formulas are all of the expressions of the forms $x=y$ and $F^{n}\left(x_{1}, \ldots, x_{n}\right)$, where $\mathrm{n}$ is a natural number. Well-formed formulas, or wffs, are then defined as the members of the smallest set $K$ containing the atomic formulas such that $\sim \phi,(\phi \rightarrow \psi)$, $(\forall x) \phi,\left(\forall F^{n}\right) \phi$ are in $\mathrm{K}$ whenever $\phi, \psi$ are in $\mathrm{K}$ and $x$ and $F^{\mathrm{n}}$ are an individual and an n-place predicate variable, respectively, for all natural numbers $n$. For convenience, we shall use ' $\phi$ ' and ' $\psi$ ' ' to refer (in the metalanguage) to wffs (with and without predicate and individual constants as well). We as- 
sume the usual notions of bondage and freedom of variables, of one variable or constant being free for another in a given wff, and of the proper substitution of a wff for an $\mathrm{n}$-place predicate variable (relative to $\mathrm{n}$ individual variables occurring free in that $\mathrm{wff}$ as subject-position indicators).

No function symbols other than predicate variables (and constants) have been introduced into our present logical syntax, it should be noted; and in this regard, it might be said, our formalism is more in line with Russell's form of logicism than with Frege's. For whereas Frege explained $\overline{ } \Delta$ predication in terms of the mathematical notion of functionality (cf. [G\&B], 47), Russell explained mathematical functionality in terms of predication, i.e., in terms of the predicative nature of propositional functions. That is, according to Russell, "the sort of function which is fundamental in logic is the propositional function; and the functions customary in mathematics are defined by means of this" (Russell, Essays in Analysis, ([EA]), 261). Thus "if $f(x)$ is not a propositional function, its value for a given value of $x \ldots$ is the term $y$ satisfying, for the given value of $x$, some relational proposition; this relational proposition is involved in the definition of $f(x)$, and some such propositional function is required in the definition of any function which is not propositional" ([POM], 508).

All functions other than propositional functions, in other words, can be identified with many-one relations (cf. $[P O M], 83$ ); and this, from either a philosophical or a logical point of view, is not an unimportant observation. Stated in this way, however, the observation in no way runs counter to Frege's form of logicism. For just as Frege analyzed all truthfunctions in terms of those for negation and the material conditional, so too he could have analyzed all functions in terms of those that he calls concepts and relations, i.e. in terms of functions from objects to truth-values. (Frege never spoke of relations as concepts; i.e., concepts were always unary functions from objects to truth-values for Frege. For convenience, however, we shall speak here of relations as relational concepts; and whether a concept is unary or relational, we shall in either case refer to the saturated object corresponding to that concept as a conceptcorrelate.)

There is something in Russell's way of making the above observation, incidentally, that should not be overlooked; namely, that functionality presupposes predication in that it depends essentially on the unity of a proposition. Curiously, though Frege himself did not explain functional- 
ity in terms of predication, nevertheless precisely this sort of consideration, whether applied to the unity of a sentence or to the thought (Gedanke) expressed by that sentence, seems to be fundamental to his notion of unsaturatedness, which of course is the basis of his notion of functionality. Thus in regard to the unsaturated nature of a predicate as the predicative component of a sentence, Frege argued that "this unsaturatedness ... is necessary, since otherwise the parts [of the sentence] do not hold together" ([PW], 177); and, similarly, "not all the parts of a thought can be complete; at least one must be 'unsaturated', or predicative; other-

$\measuredangle$ wise they would not hold together" ([G\&B], 54). In other words, though Frege explained predication in terms of his mathematical notion of functionality, ultimately his only argument for the unsaturatedness of functions is his argument for the unsaturatedness of the functions involved in predication, whether that predication be syntactical or otherwise. In this regard our present formalism neither distorts nor runs counter to Frege's form of logicism; and indeed, if anything, it rather emphasizes what is really fundamental in Frege's view of logicism.

\section{Existential Posits and the Laws of Logic}

The laws of logic, according to Frege, must be universal, but only in the sense that they must be applicable to any objects whatsoever. Frege does not mean to deny, in other words, that there are any existential posits among the laws of logic. To be sure, none of Frege's axioms in his Begriffsschrift are other than universal in form, but this does not mean that no existential posits are provable on the basis of these axioms. In particular, the (impredicative) comprehension principle,

$$
\left(\exists F^{n}\right)\left(\forall x_{1}\right) \ldots\left(\forall x_{n}\right)\left[F\left(x_{1}, \ldots, x_{n}\right) \leftrightarrow \phi\right],
$$

where $\phi$ is a wff (pure or applied) in which $F^{\mathrm{n}}$ does not occur free and $x_{1}, \ldots, x_{\mathrm{n}}$ are pairwise distinct individual variables occuring free in $\phi$, is easily seen to be provable on the basis of Frege's basic law_(IIb).

Besides being provable, however, $(\mathrm{CP})$ can be taken as an axiom schema and Frege's basic law (IIb) derived instead. That is, together with the remaining axioms and rules of standard second order predicate logic with identity, Frege's basic law (IIb), which we can formulate as follows, 
is provable on the basis of (CP) (cf. [Henkin]). Now although there is something to be said in favor of having the basic laws of logic all be universal in form, there is also something to be said for putting one's existential posits up front. In addition, there is something appropriate about avoiding the notion of proper substitution in stating the basic laws of logic-especially such a complex notion as the substitution of a wff for a predicate variable (relative to certain individual variables free in that wff as subject-position indicators).

Such an observation applies on the first order level as well, incidentally, especially if we are to adhere to Frege's view that "correctly-formed names must always denote something" ([Gg], vol. 1, section 28); for, of course, that view amounts to the assumption that logic is not free of existential presuppositions regarding singular terms. That is, where $a$ is a singular term in which $x$ does not occur (free), the fact that $a$ denotes something (as a value of the bound individual variables), i.e., $(\exists x)(a=x)$, is provable on the basis of Frege's basic law (IIa):

$$
(\forall x) \psi \rightarrow \psi(a / x) .
$$

But, conversely, given the remaining axioms and rules, $\left(\mathrm{UI}_{1}\right)$ is also provable on the basis of $(\exists x)(a=x)$ (cf. Kalish and Montague, "On Tarski's Formalization of Predicate Logic with Identity," ([K\&M])).

A substitution free axiom set for standard second order predicate logic with identity can be described, accordingly, as follows. As axioms (or basic laws of logic) we need take only all wffs (pure or applied) that are either tautologous or of one of the following forms:

$$
\begin{array}{ll}
(\forall u)[\phi \rightarrow \psi] \rightarrow[(\forall u) \phi \rightarrow(\forall u) \psi], & \text { where } u \text { is an in- } \\
& \text { dividual or a } \\
& \text { predicate vari- } \\
& \text { able, } \\
& \text { where } u \text { is an } \\
& \text { individual or } \\
& \text { predicate vari- } \\
& \text { able not occur- } \\
& \text { ring free in } \phi,
\end{array}
$$


(A3)

(CP)

$$
(\exists x)(a=x),
$$

$$
(a=b) \rightarrow(\phi \leftrightarrow \psi),
$$

where $a$ is a singular term in which $x$ does not occur free, where $a, b$ are singular terms and $\psi$ comes from $\phi$ by replacing one or more free occurrences of $b$ by free occurrences of $a$, where $F^{n}$ does not occur free in $\phi$, and $x_{1}, \ldots, x_{\mathrm{n}}$ are among the distinct individual variables occurring free in $\phi$.

As inference rules we need take only modus ponens and universal generalization:

$$
\text { if } \vdash \phi \text { and }-(\phi \rightarrow \psi) \text {, then }-\psi \text {; }
$$

if $\vdash \phi$, and $u$ is an individual or a predicate variable, then $\vdash(\forall u) \phi$.

Unlike Frege, incidentally, Russell does not take $\left(\mathrm{UI}_{1}\right)$ and $\left(\mathrm{UI}_{2}\right)$ as basic laws, but instead he takes their contrapositives, that is, their corresponding forms as existential generalizations (cf. $[P M],{ }^{* 9.1):}$

$$
\begin{array}{ll}
\left(\mathrm{EG}_{1}\right) & \psi(a / x) \rightarrow(\exists x) \psi, \\
\left(\mathrm{EG}_{2}\right) & \psi\left[\phi / F\left(x_{1}, \ldots, x_{\mathrm{n}}\right)\right] \rightarrow\left(\exists F^{\mathrm{n}}\right) \psi .
\end{array}
$$


Of course, Russell does not distinguish these two laws and expresses $\left(E G_{2}\right)$ in the form of $\left(E G_{1}\right)$; but that is because his "individual" variables are really metalinguistic variables subject to systematic ambiguity. The substitution of wffs for predicate variables that is involved in $\left(\mathrm{EG}_{2}\right)$ is only implicit in Russell, moreover, and $\left(\mathrm{EG}_{2}\right)$ is really effected through his use of the cap-notation, $\phi\left(\hat{x}_{1}, \ldots, \hat{x}_{n}\right)$, for the representation of propositional functions. Such a use of the cap-notation, needless to say, amounts to applying a syntactical operation for the generation of complex predicates. An alternative notation, which we shall adopt here, is Alonzo Church's $\lambda$-operator for functional abstraction. That is, instead of Russell's notation $\phi\left(\hat{x}_{1}, \ldots, \hat{x}_{n}\right)$ we shall use $\left[\lambda x_{1} \ldots x_{n} \phi\right]$ for the expression of a complex predicate. Russell's "primitive proposition" $\left(\mathrm{EG}_{2}\right)$ can then be restated as follows:

$$
\psi\left(\left[\lambda x_{1} \ldots x_{\mathrm{n}} \phi\right] / F^{n}\right) \rightarrow\left(\exists F^{n}\right) \psi .
$$

According to Russell, incidentally, "the above primitive proposition gives the only method of proving 'existence-theorems'" ([PM], 131). That is, "in order to prove such theorems, it is necessary (and sufficient) to find some instance in which an object possesses the property in question. If we were to assume 'existence-axioms', i.e. axioms stating $(\exists z) . \phi z$ for some particular $\phi$, these axioms would give other methods of proving existence. Instances of such axioms are the multiplicative axiom $\left({ }^{*} 88\right)$ and the axiom of infinity.... But we have not assumed any such axioms in the present work" (ibid.).

This is somewhat misleading, needless to say, since it suggests that in logic "existence-theorems" are only conditional theorems, and this we know from our discussion of (CP) is false. Thus, in particular, where $a_{1}, \ldots, a_{\mathrm{n}}$ are singular terms that are free, respectively, for $x_{1}, \ldots, x_{\mathrm{n}}$ in $\phi$, the basic law regarding complex predicates corresponding to Russell's use of his cap-notation is the principle of $\lambda$-conversion:

( $\lambda$-Conv) $\quad\left[\lambda x_{1} \ldots x_{\mathrm{n}} \phi\right]\left(a_{1}, \ldots, a_{\mathrm{n}}\right) \leftrightarrow \phi\left(a_{1} / x_{1}, \ldots, a_{\mathrm{n}} / x_{\mathrm{n}}\right)$.

Generalized, this principle can be stated as follows:

$(\forall / \lambda$-Conv $) \quad\left(\forall x_{1}\right) \ldots\left(\forall x_{n}\right)\left(\left[\lambda x_{1} \ldots x_{n} \phi\right]\left(x_{1}, \ldots, x_{n}\right) \leftrightarrow \phi\right) ;$

and of course from this, (CP) follows by $\left(\mathrm{EG}_{2}\right)$.

It is historically noteworthy, incidentally, that the first explicit use of 
(CP) as a basic law of logic occurs in [Tarski] where it is credited to Leśniewski (cf. [Henkin], 203). Leśniewski, apparently, referred to special cases of (CP), each of which is really an instance of $(\forall / \lambda$-Conv), as "pseudo-definitions," which is quite appropriate since (CP) is the logical basis of all explicit definitions of predicate constants. That is, it is on the basis of (CP) that any such definition can be shown to be noncreative and that the predicate constant so defined is eliminable in principle. It is in terms of these "pseudo-definitions," of course, that the reduction of classical mathematics to logic is to be effected.

\section{Wertverläufe as Concept-Correlates}

No reduction of mathematics is forthcoming, however, without a logistic treatment of nominalized predicates, or some such equivalent syntactic de$\Delta \Delta$ vice such as Frege's notation for value-ranges (Wertverläufe). Valueranges, it should be noted, are not sets or classes in the sense of being composed of their members; rather, they are the saturated logical objects that Frege also informally called concept-correlates. That is, value-ranges are for Frege the denotata of nominalized predicates-or at least that is what we claim in our reconstruction and shall attempt to show in what follows. If we are right in this claim, then we may in effect identify Frege's implicit logic of nominalized predicates, i.e., the logic that is implicit in his informal remarks, with his explicit logic of value-ranges. Indeed, this way of viewing Frege's theory of value-ranges, we claim, will not only provide the essential rationale for his basic law $\mathrm{V}$ regarding value-ranges but it will also explain why his theory of value-ranges is not really a second order set theory.

Now in justifying our basic claim that value-ranges are conceptcorrelates, let us turn first to Frege's paper "On Concept and Object," where Frege explicitly states that an expression of the form 'the concept $F$ ' denotes not a concept but an object that is somehow correlated with that concept. In particular, in responding to Benno Kerry's objection that concepts are objects, and, moreover, objects other than their extensions, Frege explains that "in my way of speaking expressions like 'the concept $F$ ' designate not concepts but objects" ([G\&B], 48). That is, "if he [Kerry] thinks that I have identified concept and extension of concept, he is mistaken; I merely expressed my view that in the expression 
'the number that applies to the concept $F$ is the extension of the concept like-numbered to the concept $F$ ' the words 'extension of the concept' could be replaced by 'concept'. Notice carefully that here the word 'concept' is combined with the definite article" (ibid.). In other words, according to Frege, 'the extension of the concept like-numbered to the concept $F$ ' denotes the same object as that which is denoted by 'the concept like-numbered to the concept $F$ '. In a footnote of his original draft of this passage, Frege wrote that "the question whether one should simply put 'the concept' for 'the extension of the concept' is in my view one of expediency" ([PW], 106). Of course, by the extension of a concept $V$ Frege means none other than a value-range (cf. $[G g]$, vol. 1, section 3 ).

The above explanatory remark is about as explicit as Frege gets in identifying concept-correlates with value-ranges. More indirect, but also more important, evidence for this identification can be found in the connection Frege implicitly makes between second level concepts and concept-correlates on the one hand and that which he explicitly makes between second level concepts and value-ranges on the other. A second level'A concept, we should explain, is essentially what a variable binding operator on wffs to wffs (such as a quantifier) stands for-or, equivalently, it is a concept corresponding to an open wff that may be used in a third order comprehension principle to specify such a variable binding operator, such as the wff $(\forall x)[F(x) \rightarrow G(x)]$, which specifies the second level relational concept of the subordination of one first level concept to another (cf. my "A Second Order Logic of Variable-Binding Operators," ([NBC-1])). A first level concept, i.e., one that a predicate stands for, is said by Frege to fall within a second level concept in a way analogous to (but still not the same as) the way that an object is said to fall under a first level concept.

Now the connection between second level concepts and conceptcorrelates that is implicit in "On Concept and Object" (reprinted in [G\&B]) is the thesis that corresponding to each second level concept there is a special first level concept $F$ such that a first level concept, say, $G$, falls within that second level concept if, and only if, the object correlated with $G$, i.e., the concept $G$, falls under the corresponding special first level concept F; or in symbols (in the monadic case), where subject-position occurrences of a predicate variable are nominalized occurrences of that variable:

$$
(\forall Q)(\exists F)(\forall G)[(Q x) G(x) \leftrightarrow F(G)] .
$$


Thus, for example, corresponding to the second level concept of (objectual) existence, i.e., the second level concept that first order existential quantifier phrases stand for, there is the special first level concept of being realized; and the correspondence, according to Frege, is so tight that even the same thought is expressed by 'there is a square root of 4' 'and 'the concept square root of 4 is realized' (cf. [GむB], 49f). A conceptcorrelate is realized, in other words, if, and only if, there exists an object that falls under the concept in question.

Needless to say, but if value-ranges really are concept-correlates, then Frege's thesis connecting second level concepts with concept-correlates should connect second level concepts with value-ranges; and indeed Frege explicitly states this to be the case in $[G g]$, vol. 1 , section 25 . That is, according to Frege, "second level functions can be represented in a certain manner by first level functions, whereby the functions that appear as arguments of the former are represented by their value-ranges" (op. cit.); or in symbols (in the case of unary concepts):

$$
(\forall Q)(\exists F)(\forall G)\left[(Q x) G(x) \leftrightarrow F\left(\epsilon^{\prime} G(\epsilon)\right)\right] .
$$

The singular term $\dot{\epsilon}^{\prime} G(\epsilon)$ is of course Frege's notation for the extension (value-range) of the concept $G$; and the above thesis, it should be noted, amounts in effect to a restatement of Frege's context principle regarding such expressions. That is, just as it is only in the context of a sentence (or of a wff in general) that a predicate can occur as a predicate, it is only through a correlation with such occurrences of a predicate that we are to understand the role of the name for a value-range; and of course it is precisely the same thesis, and therefore the same restatement of his context principle, that Frege implicitly gives for nominalized predicates, i.e., for abstract singular terms of the form 'the concept $G$ '. We may read ' $\epsilon$ ' $G(\epsilon)$ ', in other words, either as 'the concept G' or as 'the extension of the concept $G$ ', which, as already noted, is exactly what Frege said in the footnote referred to above ([PW], 106).

For convenience, we shall hereafter refer to the above thesis (in either form) as Frege's double correlation thesis. This is because Frege assumes as part of his thesis both a one-to-one correlation between second level concepts and certain special first level concepts on the one hand, and a one-to-one correlation between first level concepts and certain special objects called concept-correlates or value-ranges on the other. Our two alter- 
native reconstructions or resurrections of Frege's logicism, as we shall see, will turn precisely on a minor modification of one or the other of the correlations involved in this thesis.

\section{Frege's Double Correlation Thesis and His Basic Law V}

There is at least one other place in Frege's writings that clearly indicates that value-ranges are concept-correlates, and that given Frege's extensional view of concepts (as functions from objects to truth-values) provides the essential rationale for his basic law V. Both the identification and the rationale, it should be noted, are again based on Frege's double correlation thesis, only now applied to second level relational concepts, and in particular to the second level relation of mutual subordination.

Indeed, Frege's basic law V, viz.,

$$
\epsilon^{\prime} F(\epsilon)=\epsilon^{\prime} \xi \dot{\xi} G(\epsilon) \leftrightarrow(\forall x)[F(x) \leftrightarrow G(x)],
$$

should be viewed precisely as a special instance of his double correlation thesis. For what is indicated on the right hand side of this law is none $\checkmark$ other than the second level relation of material equivalence or mutual subordination of two first level concepts; and on Frege's extensional view of concepts (as functions from objects to truth-values) such an equivalence amounts in effect to their "identity." That is, Frege's basic law V amount $\hat{\Delta} \Delta$ to correlating the first level relation of identity with his second level relation of mutual subordination. Such a correlation is needed, Frege observes, since "to construe mutual subordination simply as equality is forbidden by the basic difference between first and second level relations. Concepts cannot stand in a first level relation. That wouldn't be false, it would be nonsense. Only in the case of objects can there be any question of equality (identity). And so the said transformation [from mutual subordination to identity] can only occur by concepts being correlated with the same object. It is all, so to speak, moved down a level" ([PW], 182, italics added). Of course, if concept-correlates are identical when the concepts in question are mutually subordinate, then concept-correlates are none other than the extensions of the correlated concepts; i.e., then conceptcorrelates are value-ranges.

The identification of value-ranges as concept-correlates also explains, it should be noted, why Frege's theory of value-ranges is really not a sec- 
$\therefore \therefore$ ond order set theory. For as a concept-correlate, Frege observes, a valuerange "simply has its being in the concept, not in the objects which belong to it" (ibid., 183). That is, unlike sets whose existence or being is constituted by their members, concept-correlates, and therefore valueranges, are "logical objects" whose determination is given by Frege's double correlation thesis, which, as already noted, is really a restatement of his context principle applied specifically to nominalized predicates. It is this thesis that explains how "by means of our logical faculties we lay hold upon the extension of a concept, by starting out from the concept" (ibid., 181); for, as already noted, a value-range, and therefore a concept-correlate, is none other than the extension of a concept. In this regard, accordingly, the confusion sometimes made of Frege's theory of value-ranges with a second order set theory might best be obviated by directly describing his theory of value-ranges as a theory of concept-correlates; that is, by describing his logicism as a second order predicate logic with nominalized predicates.

\section{Russell and Frege on Nominalized Predicates}

$\Delta$ U' Unlike Frege, for whom the extension of a concept "has its being in the concept, not in the objects which belong to it," Russell originally took the extension of a concept to be a class, or rather he took it to be what he called a class as many as opposed to a class as one; and a class as many, according to Russell, is essentially many, i.e., it is essentially composed of its members (cf. [POM], chap. 6). Being many, however, a class as many is not a single object, according to Russell, and therefore it could not occur in a proposition as a term, which in effect defeated the whole point of Russell's original form of logicism.

Of course, prior to the discovery of his paradox, Russell assumed that a class as one always existed corresponding to a class as many (cf. [POM], 104); and as so determined, a class as one was also composed of its members. But since a class as one is an individual, according to Russell, then it, unlike its corresponding class as many, can occur in a proposition as a term; and therefore the fact that a class as many, i.e., the extension of a 
concept, was not a single object was without any real effect in Russell's original form of logicism.

With the discovery of his paradox, however, Russell gave up the assumption that a class as one always existed corresponding to a class as many. That is, Russell came to believe that it was this assumption that was "the source of the contradiction" (ibid.). In particular, in regard to his paradox of the class of classes that are not members of themselves, Russell found that he could only conclude that "the classes which as ones are not members of themselves as many do not form a class-or rather, that they do not form a class as one, for the argument cannot show that they do not form a class as many" (ibid., 102). Having given up this as- $\Delta$ sumption, however, Russell in effect was forced to give up his original form of logicism.

Shortly after completing $[P O M]$, Russell gave up not only the assumption that a class as one always existed corresponding to a class as many, but he gave up assuming that there are any classes at all-i.e., classes in the sense of objects that are essentially composed of their members. Thus, in his letter of 24 May 1903 to Frege we find Russell writing, "I believe I have discovered that classes are entirely superfluous. Your designation $\dot{\epsilon}^{\prime} \phi(\epsilon)$ can be used for $\phi$ itself, and $x \cap \dot{\epsilon} \phi(\epsilon)$ for $\phi(x)$ " (Frege, Philosophical and Mathematical Correspondence, ([PMC]), 158). (Frege's notation ' $x \cap \dot{\epsilon} \phi(\epsilon)$ ' in effect amounts to ' $x \in \dot{\epsilon} \boldsymbol{\phi}(\epsilon)$ ', as Russell observed in [POM], 512.) The important suggestion here is that propositional functions are individuals after all, and therefore they can occur in propositions as terms instead of the classes as ones Russell originally assumed to correspond to their extensions. That is, instead of assuming that there are any classes, whether as "ones" or as "manys," Russell is now proposing what later he called his "no classes" theory; i.e., the theory that propositional functions are single entities (individuals) after all, and that all talk of classes is to be reduced to talk of propositional functions.

Frege's response to Russell's letter is of course predictable. "I cannot regard your attempt to make classes entirely dispensable as successful, the reason being that you use function letters in isolation" ([PMC], 160). In other words, according to Frege, "to use a function sign in isolation is to contradict the nature of a function, which consists in its unsaturatedness" (ibid.). Russell's reply in turn is, of course, also predictable by now, for he 
writes, "It is not clear to me that it is never permissible to use a function letter in isolation" (ibid., 166). That is, Russell simply refuses to accept the unsaturated nature of concepts.

Despite the stalemate on this point, however, the exchange is instructive for our present purposes. For to do what Russell suggests, Frege (ibid., 161) observes that "we would first have to transform all [unary] function names in such a way that there was only one argument place and that was on the right-hand side. Thus, we would have to transform, e.g., ' $x^{2}=1$ ' into ' $\varepsilon\left(\epsilon^{2}=1\right) x$ ', and ' $x(x-1)(x+1)=0$ ' into ' $\varepsilon[\epsilon(\epsilon-1)(\epsilon+1)$ $=0] x$ ', so that we could write

$$
\dot{\epsilon}\left(\epsilon^{2}=1\right) x_{x}^{\supset} \hat{\epsilon}[\epsilon(\epsilon-1)(\epsilon+1)=0] x
$$

and for this, according to your [Russell's] definition,

$$
\dot{\epsilon}\left(\epsilon^{2}=1\right) \subset \hat{\epsilon}[\epsilon(\epsilon-1)(\epsilon+1)=0] . ”
$$

Now what should be especially noted here is that Frege uses the spiritus asper or rough breathing operator to generate a complex predicate as opposed to his use of the spiritus lenis or smooth breathing operator to generate a complex singular term (for a value-range). Stated in terms of our own notation, and using the $\lambda$-operator to generate complex predicates, Frege's point is that in transforming a wff $\phi(x)$ into a complex predicate, $[\lambda x \phi(x)]$, we must keep in mind that $[\lambda x \phi(x)]$ really has a pair of parentheses (and commas as well in the case of a relational predicate) accompanying it. Thus whereas

$$
(\forall y)([\lambda x \phi(x)](y) \rightarrow[\lambda x \psi(x)](y))
$$

is a well-formed formula in which two $\lambda$-abstracts occur as complex predicates, the expression

$$
R([\lambda x \phi(x)],[\lambda x \psi(x)]),
$$

where $R$ is a 2-place predicate constant, cannot be well-formed or meaningful as far as Frege is concerned, since as a complex predicate a $\lambda$-abstract "would be defined only in connection with an argument sign following it, and it would nevertheless be used without one; it would be defined as a function sign and used as a proper name, which will not do" (ibid., 161f).

In his reply on this point, Russell is undaunted, insisting, as already noted, that an expression for a propositional function can at least in some 
cases be used as a singular term. This will not do as it stands, of course, but we can reconstruct Russell's position here by distinguishing between two transformations of a wff $\phi(x)$ where Frege has acknowledged only one. That is, we can first transform $\phi(x)$ into the complex predicate $[\lambda x \phi(x)]()$, which does have an accompanying argument or subject position (indicated by the last pair of parentheses); and then we can transform this complex predicate into the singular term $[\lambda x \phi(x)]$, which does not have an accompanying argument or subject position. A confusion might arise here if we allow ourselves to speak of $[\lambda x \phi(x)]$ as both a predicate and a singular term; but so long as we understand that when used as a predicate it must be accompanied by a pair of parentheses (and commas in the case of a relational predicate), no confusion should arise if these parentheses (and commas) are informally dropped for abbreviatory purposes.

Frege, incidentally, is not unaware of this second transformation of a predicate into a singular term; for in applying Russell's suggested notation in just this way, he notes that "we would have ' $\varepsilon\left(\epsilon^{2}=1\right)=\varepsilon[(\epsilon-$ $1)(\epsilon+1)=0]$ ', which does not differ essentially from my ' $\epsilon$ ( $\left.\epsilon^{2}=1\right)=\epsilon^{2}[(\epsilon$ $-1)(\epsilon+1)=0$ ]' " (ibid., 162). But in that case, Frege observes, Russell's suggested notation for nominalized predicates "would lead to the same difficulties as my value-range notation" (ibid., 161). That is, other than assuming that nominalized complex predicates denote concepts or propositional functions as single entities, Russell's suggested use of such singular terms in 1903 was no less immune from his paradox than was Frege's use of the same singular terms to denote value-ranges.

\section{Russell's Paradox Revisited}

In turning to Russell's paradox and its resolution in our reconstruction of Frege's and Russell's early form of logicism, i.e., the form of logicism Russell was implicitly advocating in his correspondence with Frege, let us first give a more explicit formulation of both the logical grammar and the logical principles involved in the above exchange. As indicated, instead of using both the spiritus asper, or rough breathing operator, for the generation of complex predicates from wffs and the spiritus lenis, or smooth breathing operator, for the generation of complex singular terms from wffs, we shall follow the reconstructed Russellian strategy suggested above and use only the $\lambda$-operator for both purposes. Also, for convenience of expression we 
shall informally drop parentheses and commas when referring to predicates. It should be noted in this regard, however, that using one operator and adopting an informal convention of dropping parentheses and commas when referring to predicate expressions in no way prejudges the case in favor of Russell's view that concepts have a saturated or individual nature. Aside from simplicity and economy of notation, in other words, the convention allows us to formulate a logical grammar that is common to both forms of logicism. (We will later introduce an intensional operator for Russell's form of logicism as well, but this will not affect the grammar that is common to both forms of logicism.)

In describing our logical grammar we shall for convenience of exposition identify the different types of meaningful expressions by associating them with different natural numbers, where 0 is understood to represent the type of all singular terms, 1 the type of all wffs or propositional forms, and $\mathrm{n}+1$, for $\mathrm{n}>1$, the type of all $\mathrm{n}$-place predicate expressions. Individual variables, accordingly, are of type 0 , propositional variables are of type 1 , and n-place predicate variables are of type $n+1$. We continue to ignore the introduction of special individual and predicate constants, and, for $\mathrm{n} \in \omega$, we recursively define the meaningful expressions of type $\mathrm{n}$, in symbols, $\mathrm{ME}_{\mathrm{n}}$, as follows:

every individual variable (or constant) is in $\mathrm{ME}_{0}$, and every $\mathrm{n}$-place predicate variable (or constant) is in both $\mathrm{ME}_{\mathrm{n}+1}$ and $\mathrm{ME}_{0}$;

if $a, b \in \mathrm{ME}_{0}$, then $(a=b) \in \mathrm{ME}_{1}$;

if $\pi \in \mathrm{ME}_{\mathrm{n}+1}$, and $a_{1}, \ldots, a_{\mathrm{n}} \in \mathrm{ME}_{0}$, then $\pi\left(a_{1}, \ldots, a_{\mathrm{n}}\right) \in$ $\mathrm{ME}_{1}$;

if $\phi \in \mathrm{ME}_{1}$, and $x_{1}, \ldots, x_{\mathrm{n}}$ are pairwise distinct individual variables, then $\left[\lambda x_{1} \ldots x_{n} \phi\right] \in \mathrm{ME}_{\mathrm{n}+1}$;

if $\phi \in \mathrm{ME}_{1}$, then $\sim \phi \in \mathrm{ME}_{1}$;

if $\phi$ and $\psi$ are in $\mathrm{ME}_{1}$, then $(\phi \rightarrow \psi) \in \mathrm{ME}_{1}$;

if $\phi \in \mathrm{ME}_{1}$, and $a$ is an individual or a predicate variable, then $(\forall a) \phi \in \mathrm{ME}_{1}$;

if $\phi \in \mathrm{ME}_{1}$, then $[\lambda \phi] \in \mathrm{ME}_{0}$; and

if $\mathrm{n}>1$, then $\mathrm{ME}_{\mathrm{n}} \subseteq \mathrm{ME}_{0}$. 
Singular terms, which we shall also refer to simply as terms, are now understood to be all the members of $\mathrm{ME}_{0}$; and for $\mathrm{n}>0$, we understand the members of $\mathrm{ME}_{\mathrm{n}+1}$ to be $\mathrm{n}$-place predicate expressions. We are in general to think of each $\mathrm{n}$-place predicate expression as having $\mathrm{n}$ argument or subject positions associated with it, and, as in clause (3) above, these are all understood to occur within parentheses and to be separated from one another by commas. Wffs or propositional forms are, of course, all members of $\mathrm{ME}_{1}$. Note that whereas by clause (9) every predicate expression is a term, not every wff is a term. We differ in this regard from what Frege would allow; but our difference is negligible since by clause (4), where $n$ $=0,[\lambda \phi]$ is a wff if $\phi$ is a wff, and by clause $(8)[\lambda \phi]$ is a term. In other words, besides 0 -place predicate variables (and constants), wffs are terms only when prefixed by the $\lambda$-operator. We shall in general read ' $[\lambda \phi]$ ' as 'that $\phi$ ' when it occurs in a wff as a term, i.e., when it occurs in one of the argument or subject positions of a predicate expression.

It is clear, of course, that predicate expressions occurring in the argument or subject positions of other predicates, or of themselves as well, are intended to represent the nominalized predicates that occur in natural language. For this reason, we shall refer to such occurrences of a predicate as nominalized occurrences of that predicate, acknowledging thereby that it has been transformed into an abstract singular term. Note that adding such suffixes as '-ity', '-ness', or '-hood' to nominalized occurrences of predicates would be completely superfluous here since such occurrences are already formally identified as subject position occurrences. The same observation applies, needless to say, to the use of such related phrases as 'the concept $F$ ' or 'being an $F$ '. Such phrases and suffixes are important in transformational grammar, no doubt, since they serve to mark derived nominal expressions in the surface grammar of English; and in that regard we shall ourselves use such expressions when translating or verbally stating certain theses in English. Nevertheless, it is sheer sophistry to insist that such surface grammatical features of English either must or should occur in our "deep structure" logical forms, as though a logical error were being committed otherwise.

As logical principles regarding this grammar and with respect to which Russell's paradox is to be derived, we assume exactly the same axioms and inference rules of standard second order predicate logic with identity already described in section 3, but understood now to apply to wffs con- 
taining nominalized predicates as well. We assume in this regard (but avoid going into the details here) the obvious definitions of bondage and freedom of terms and predicate expressions in wffs and $\lambda$-abstracts, and also when one such expression can be properly substituted for another of the same type. In general we shall use a "-label in referring to axioms and other theses so as to remind ourselves that we are now dealing with wffs that may contain nominalized predicates as singular terms. Thus, e.g., axiom (A3), which is now referred to as (A3"), has not only

$$
(\exists y)\left(F^{n}=y\right)
$$

but also

$$
(\exists y)\left(\left[\lambda x_{1} \ldots x_{\mathrm{n}} \phi\right]=y\right)
$$

as an instance. Similarly, Leibniz's law, (LL), which is now referred to as $(\mathrm{LL} *)$, has not only

$$
F^{\mathrm{n}}=G^{\mathrm{n}} \rightarrow\left(\forall y_{1}\right) \ldots\left(\forall y_{\mathrm{n}}\right)\left[F\left(y_{1}, \ldots, y_{\mathrm{n}}\right) \leftrightarrow G\left(y_{1}, \ldots, y_{\mathrm{n}}\right)\right]
$$

but also

$$
\begin{aligned}
& {\left[\lambda x_{1} \ldots x_{n} \phi\right]=\left[\lambda x_{1} \ldots x_{n} \psi\right] \rightarrow\left(\forall y_{1}\right) \ldots\left(\forall y_{n}\right)\left(\left[\lambda x_{1} \ldots\right.\right.} \\
& \left.\left.x_{n} \phi\right]\left(y_{1}, \ldots, y_{n}\right) \leftrightarrow\left[\lambda x_{1} \ldots x_{n} \psi\right]\left(y_{1}, \ldots, y_{n}\right)\right)
\end{aligned}
$$

as an instance. Together with $(\lambda$-Conv"), moreover, this last instance of $\left(\mathrm{LL}^{*}\right)$ has the following generalized form of Frege's basic law $(\mathrm{Vb})$ as a consequence:

$$
\left[\lambda x_{1} \ldots x_{n} \phi\right]=\left[\lambda x_{1} \ldots x_{n} \psi\right] \rightarrow\left(\forall x_{1}\right) \ldots\left(\forall x_{n}\right)(\phi \leftrightarrow \psi\rangle .
$$

Note incidentally that the comprehension principle (CP), now referred to as (CP"), does not have the following standard formulation of Russell's paradox as an instance:

$$
(\exists F)(\forall G)[F(G) \leftrightarrow \sim G(G)] .
$$

This is because concepts are posited by $\left(\mathrm{CP}^{* *}\right)$ only by means of conditions that apply to all individuals or objects, whether those individuals be abstract or concrete. It is for exactly the same reason, moreover, that one cannot define in this context a predicate, say 'Impredicable', as follows:

$$
(\forall G)[\text { Impredicable }(G) \leftrightarrow \sim G(G)] .
$$


For, as already indicated, an explicit definition of a predicate constant must be based upon the comprehension principle $\left(\mathrm{CP}^{*}\right)$ in the sense of being one of its existential instantiations, and the above fails in this regard for the same reason that the preceding wff is not an instance of $\left(\mathrm{CP}^{*}\right)$.

Russell does give another version of his paradox, however, in terms of "what seems like a complex relation, namely the combination of nonpredicability with identity" ([POM], 97); and this version is an instance of $\left(\mathrm{CP}^{*}\right)$ :

$$
(\exists F)(\forall x)(F(x) \leftrightarrow(\exists G)[x=G \& \sim G(x)]) .
$$

A contradiction is derivable from this instance of $\left(\mathrm{CP}^{*}\right)$, it should be noted, only because

$$
(\forall x) \phi \rightarrow \phi(F / x)
$$

is derivable from $\left(\mathrm{A} 3^{*}\right)$ and $\left(\mathrm{LL}^{*}\right)$. (By (LL"),

$$
F=x \rightarrow[\phi \rightarrow \phi(F / x)],
$$

and therefore by (UG), (A1*), (A2") and tautologous transformations,

$$
(\exists x)(F=x) \rightarrow[(\forall x) \phi \rightarrow \phi(F / x)] .
$$

(UI $\left.I_{i}^{*}\right)$ then follows, accordingly, by (A3") and modus ponens.) Indeed, by weakening either $\left(\mathrm{A} 3^{*}\right)$ or $\left(\mathrm{LL}^{*}\right)$ in certain obvious ways it can be shown that the resulting second order logic of nominalized predicates is consistent, not inconsistent. The weakened version of $\left(\mathrm{LL}^{*}\right)$ is appropriate, it should be noted, only when identity is defined (or replaced) by indiscernibility. (Cf. my Logical Investigations of Predication Theory and the Problem of Universals, ([NBC-4]), section 4.6 for a consistency proof when $\left(\mathrm{LL}^{*}\right)$ is weakened to its version for indiscernibility; and also section 4.10 for a consistency proof when (A3*) is weakened instead.) We do not contemplate rejecting or modifying $\left(\mathrm{LL}^{*}\right)$ here, however, and although $\left(\mathrm{A} 3^{*}\right)$ will be weakened in our second alternative reconstruction of Frege's logicism, we shall retain it in our first and more fundamental reconstruction, since, together with (LL*), it implies the part of Frege's double correlation thesis, namely that every concept has a unique saturated conceptcorrelate, that will remain intact in our first reconstruction of Frege's logicism. Of course, since this correlation for Russell is really an identity 
then $\left(\mathrm{A}^{*}\right.$ ) cannot be weakened in any reconstruction of Russell's early form of logicism.

Now in his correspondence with Frege, Russell does suggest weakening one other principle, namely the comprehension principle ( $\left.\mathrm{CP}^{*}\right)$. Thus, in his letter of 12 December 1904 to Frege, we find Russell writing, "I believe that contradiction does not arise from the nature of a class, but from the fact that certain expressions of the form

$$
(\phi) \cdot \mathrm{F}(x, \phi x, \phi \xi)
$$

$\ldots$ do not represent [propositional] functions of $x$. That is, we have

$$
\text { 1-::( } \exists F):: \sim(\exists f): .(x): f x \equiv(\phi) \cdot F(x, \phi x, \phi \xi) .
$$

This is easy to prove in the case of

$$
x=\xi(\phi \xi) \cdot \stackrel{\supset}{\supset} \cdot \sim \phi x .
$$

For this proposition denies $f\{\xi(f \xi)\}$ for any $f^{\prime \prime}([P M C], 167)$. Restated in our own present notation, Russell's particular example amounts to

$$
(\forall G)[x=[\lambda y G(y)] \rightarrow \sim G(x)],
$$

and given the identity

$$
G=[\lambda y G(y)]
$$

as a law of the logic of nominalized predicates, Russell's particular instance of $\left(\mathrm{CP}^{*}\right)$ that he wants to reject is

$$
(\exists F)(\forall x)(F(x) \leftrightarrow(\forall G)[x=G \rightarrow \sim G(x)]),
$$

which in the present context is also easily seen to lead to a contradiction. The editors of $[P M C]$ erroneously claim that there is no contradiction here (cf. p. 168), incidentally, and their explanation suggests that they are ignoring Russell's original proposal that $f=\xi(f \xi)$. But even without this particular identity, it is clear that a contradiction is derivable on the basis of $\left(\mathrm{LL}^{\prime \prime}\right)$, or what amounts to Frege's basic law $(\mathrm{Vb})$, and the following instance of $\left(\mathrm{CP}^{*}\right)$, which is clearly intended by Russell in the letter in question:

$$
(\exists F)(\forall x)(F(x) \leftrightarrow(\forall G)[\mathrm{x}=[\lambda y G(y)] \rightarrow \sim G(x)]) .
$$


Now it is clear of course that Russell is not proposing that we are to reject all instances of $\left(\mathrm{CP}^{*}\right)$ in his letter to Frege, but only those of a certain form. What is not clear, on the other hand, is the precise delimitation of the excluded forms in question; that is, it is not clear what restricted form $\left(\mathrm{CP}^{*}\right)$ is to have according to Russell. What we shall suggest in our reconstruction of this early form of Russell's logicism is that $\left(\mathrm{CP}^{*}\right)$ is to be restricted in accordance with the theory of simple types Russell described in Appendix B of $[P O M]$, though applied now to propositional functions as individuals rather than to classes as many as the extensions of propositional functions. Such a theory, in other words, seems to be what Russell had in mind in his correspondence with Frege shortly after the completion of $[P O M]$.

Before turning to the specifics of Russell's proposal, however, let us note that independently of its contradictory instances $\left(\mathrm{CP}^{*}\right)$ is a consequence of a still simpler form of comprehension principle, namely,

$$
\left(\exists F^{n}\right)\left(\left[\lambda x_{1} \ldots x_{n} \phi\right]=F\right),
$$

where $F^{n}$ does not occur free in $\phi$. For by $\left(\mathrm{LL}^{*}\right)$ and $\left(\forall / \lambda\right.$-Conv $\left.{ }^{*}\right)$,

$$
\left[\lambda x_{1} \ldots x_{\mathrm{n}} \phi\right]=F \rightarrow\left(\forall x_{1}\right) \ldots\left(\forall x_{\mathrm{n}}\right)\left[F\left(x_{1}, \ldots, x_{\mathrm{n}}\right) \leftrightarrow \phi\right],
$$

from which $\left(\mathrm{CP}^{*}\right)$ follows by (UG), $\left(\mathrm{A} 1^{*}\right)$, tautologous transformations and $\left(\mathrm{CP}_{\lambda}^{*}\right)$. A restricted form of $\left(\mathrm{CP}_{\lambda}^{*}\right)$, needless to say, will imply only a restricted form of $\left(\mathrm{CP}^{*}\right)$; and for this reason we shall apply our reconstruction of Russell's proposal to $\left(\mathrm{CP}_{\lambda}^{*}\right)$ instead.

\section{Frege's Rejection of Schröder's Hierarchy of Individuals}

Four months prior to his completion of [POM] in December 1902, Russell wrote Frege suggesting that "the contradiction could be resolved with the help of the assumption that ranges of values are not objects of the ordinary kind; i.e., that $\phi(x)$ needs to be completed (except in special circumstances) either by an object or by a range of values of objects or by a range of values of ranges of values, etc. This theory," Russell observed, "is analogous to your theory about functions of the first, second, etc. levels. In $x \cap u$ it would be necessary that $u$ was the range of values of ob- 
jects of the same degree as $x: x \cap x$ would therefore be nonsense. This view would also be useful in the theory of relations" ([PMC], 144). This suggestion was subsequently described as the simple theory of types that appears in Appendix B of [POM].

In his reply to Russell and the suggestion "that we are to conceive of ranges of values and hence also of classes as a special kind of objects whose names cannot appear in all argument places of the first kind" (ibid., 145), Frege noted that "a class would not then be an object in the full sense of the word, but-so to speak-an improper object for which the law of excluded middle did not hold because there would be predicates that could be neither truly affirmed nor truly denied of it. Numbers would then be improper objects" (ibid., italics added). In his discussion of Russell's paradox in the appendix to volume 2 of $[G g]$, Frege reiterated this objection, noting that if "classes were proper objects, the law of excluded middle would have to hold for them" ([G\&B], 235).

Now it is noteworthy that in 1895 Frege had already considered and rejected the restriction on the laws of logic that would be necessary for the kind of hierarchy of classes (and later of propositional functions as individuals) Russell was suggesting as a way of avoiding his paradox. The circumstances of this rejection had nothing to do with Russell's paradox, needless to say, since Frege was at that time unaware of the paradox; but rather they had to do with Ernst Schröder's conceptual difficulties with the empty set as an extension consisting of nothing on the one hand, and with his notion of a singleton as an extension that was identical with its only member on the other (cf. [G\&B], 86-106). Be that as it may, Schröder, as Alonzo Church has observed, anticipated the theory of types when he took "the universal class 1 which appears in his algebra, not as an absolute universal class, but as composed of all the elements of a certain domain fixed in advance" ([Church], 150). Once such a universal class or "manifold" was given, moreover, a second may be obtained (to which the algebra is to be applied in turn) "by taking the subsets of the first to be the individuals of the second" (ibid.); and by continuing in this way a "hierarchy of reine Mannigfaltigkeiten may be extended to infinity" (ibid.). The crucial restriction Schröder imposed on this hierarchy was that no subset of the domain of "individuals" considered at any stage of the hierarchy was to be among the individuals of that stage, and that consequently the laws of logic, as the laws of his algebra, were to be re- 
stricted in any given application only to the individuals of the stage in question.

Now it was precisely this sort of restriction of the "field for our logical activities" ([G\&B], 92) that Frege criticized and rejected in his 1895 review of Schröder's book. For "whereas elsewhere logic may claim to have laws of unrestricted validity, we are here required to begin by delimiting a manifold with careful tests, and it is only then that we can move around inside it" (ibid.).

Russell was on the mark, it should be noted, when he observed that such a restriction on the laws of logic was already imposed by Frege on his functions or concepts of first, second, etc., levels. But then for Russell, it must be remembered, these functions or concepts do not have an unsaturated nature; and as far as Russell was concerned, if the laws of logic can be restricted when applied to concepts of different levels, then they can also be restricted when applied to the extensions of those concepts as well. Indeed, once classes as the extensions of concepts were eliminated from logic altogether, then, as far as Russell was concerned, the restrictions in question are essentially the restrictions already imposed by Frege on the concepts of different levels.

Now the crucial point in Russell's view of Frege's hierarchy, it must be emphasized, is that concepts do not have an unsaturated nature; i.e., that despite their predicative nature (as functions from individuals to propositions), concepts for Russell are abstract individuals. This is essential to Russell's interpretation, since if concepts really do have an unsaturated nature as Frege claims, then they cannot be construed as objects or abstract (higher order) individuals; and as a theory of different types of individuals, the theory of types, whether simple or ramified, would then not be applicable to Frege's concepts of different levels. Indeed, it is precisely because concepts, and functions in general, have an unsaturated nature according to Frege that Church rejects "the claim sometimes made on behalf of Frege that his Stufen ... constitute an anticipation of the simple theory of types" ([Church], 151). And in his rejection of classes as "improper objects" Frege himself points out that "there is nothing 'unsaturated' or predicative about classes that would characterize them as functions, concepts, or relations" ([G\&B], 235). Numbers in particular are objects, according to Frege, not concepts or "improper objects" as they would have to be on Russell's proposal. 


\section{Frege's Double Correlation Thesis and the Theory of Simple Logical Types}

There is a way, it turns out, of reconstructing Russell's proposal while still agreeing with Frege that if nominalized predicates denote objects as individuals "in the full sense," then the laws of logic, and the law of excluded middle in particular, must not be restricted when applied to such objects. We shall do so, moreover, by applying the notion of a simple logical type not to a description of Frege's Stufen or levels of concepts as "improper objects" the way Russell suggests, but rather as a description of the conditions determined by Frege's double correlation thesis for positing first level concepts and their corresponding concept-correlates.

Now the point of our reconstruction is that if second level concepts can be correlated with certain first level concepts, then third level concepts can be similarly correlated with second level concepts, and therefore, by the product of these correlations, third level concepts can in effect also be correlated with first level concepts. Similarly, fourth level concepts can be correlated with third level concepts and therefore with first level concepts as well. In general, in other words, all concepts of whatever level can in effect be correlated with first level concepts, and these in turn can be correlated with certain (saturated) objects called concept-correlates. Thus instead of speaking of a higher level concept $Q$ falling within a concept $M$ of one level higher we shall instead generalize Frege's double correlation thesis and speak of the concept-correlate of the first level concept corresponding to $Q$ as falling under the first level concept corresponding to $M$. Doing so, however, requires that the conditions for specifying the first level concepts and concept-correlates in question must be stratified in a way corresponding to the stratification of the higher level concepts to which these first level concepts and their concept-correlates correspond. The comprehension principle $\left(\mathrm{CP}_{\lambda}^{*}\right)$, in other words, must be restricted in a way that corresponds to the stratification of the unsaturated concepts of Frege's hierarchy.

We shall actually need a more stringent form of stratification than Frege allows, it turns out, for reasons shown below, and specifically one for which it is assumed that there are no unequal higher level relations (or at least none that are involved in Frege's double correlation thesis); that is, we shall be required to assume, on pain otherwise of generating Russell's paradox after all, that higher level relations are only homoge- 
neously stratified. This, in fact, will be the only modification of Frege's original form of logicism that we shall assume in our first reconstruction of Frege's logic.

Returning to the logical grammar of section 7, accordingly, we shall say that a formula or $\lambda$-abstract $\phi$ of that grammar is homogeneously stratified if, and only if, there is an assignment $t$ of natural numbers to the set of terms and predicate expressions occurring in $\phi$ (including $\phi$ itself if $\phi$ is a $\lambda$ abstract) such that (1) for all terms $a$ and $b$, if ( $a=b)$ occurs in $\phi$, then $\mathrm{t}(a)$ $=\mathrm{t}(b) ;(2)$ for all $\mathrm{n} \geq 1$, all $\mathrm{n}$-place predicate expressions $\pi$ and all terms $a_{1}, \ldots, a_{\mathrm{n}}$, if $\pi\left(a_{1}, \ldots, a_{\mathrm{n}}\right)$ is a wff occurring in $\phi$, then (i) $\mathrm{t}\left(a_{\mathrm{j}}\right)=\mathrm{t}\left(a_{\mathrm{k}}\right)$, for 1 $\leq \mathrm{j}, \mathrm{k} \leq \mathrm{n}$, and (ii) $\mathrm{t}(\pi)=\mathrm{t}\left(a_{1}\right)+1$; and (3) for all $\mathrm{m} \in \omega$, all individual variables $x_{1}, \ldots, x_{\mathrm{m}}$, and all wffs $\psi$, if $\left[\lambda x_{1} \ldots x_{\mathrm{m}} \psi\right]$ occurs in $\phi$, then (iii) $\mathrm{t}\left(x_{\mathrm{j}}\right)=$ $\mathrm{t}\left(x_{\mathrm{k}}\right)$, for $1 \leq \mathrm{j}, \mathrm{k} \leq \mathrm{m}$, and (iv) $\mathrm{t}\left(\left[\lambda x_{1} \ldots x_{\mathrm{m}} \psi\right]\right)=\mathrm{t}\left(x_{1}\right)+1$. If clauses (i) and (iii) are dropped and clauses (ii) and (iv) are replaced by the weaker requirement that $\mathrm{t}(\pi)=1+\max \left[\mathrm{t}\left(a_{1}\right), \ldots, \mathrm{t}\left(a_{\mathrm{n}}\right)\right]$ and $\mathrm{t}\left(\left[\lambda x_{1} \ldots x_{\mathrm{m}} \psi\right]\right)=1+$ $\max \left[\mathrm{t}\left(x_{1}\right), \ldots, \mathrm{t}\left(x_{\mathrm{m}}\right)\right]$, then we shall say that $\phi$ is heterogeneously stratified, or simply stratified; and if clause (1) as well as clauses (i) and (iii) are dropped and clauses (ii) and (iv) are replaced by the still weaker requirement that $\max \left[\mathrm{t}\left(a_{1}\right), \ldots, \mathrm{t}\left(a_{\mathrm{n}}\right)\right]<\mathrm{t}(\pi)$ and $\max \left[\mathrm{t}\left(x_{1}\right), \ldots, \mathrm{t}\left(x_{\mathrm{m}}\right)\right]<$ $\mathrm{t}\left(\left[\lambda x_{1} \ldots x_{\mathrm{m}} \psi\right]\right)$, then we shall say that $\phi$ is cumulatively stratified.

We include the idea of cumulative stratification here because it is a natural generalization of the idea of a stratified hierarchy of concepts. Involved in such a generalization, in other words, is the suggestion that we should restrict $\left(\mathrm{CP}_{\lambda}^{*}\right)$ as little as possible in our representation of Frege's double correlation thesis; that is, we should replace $\left(\mathrm{CP}_{\lambda}^{*}\right)$ by the cumulatively stratified comprehension principle, $\left(\mathrm{CSCP}_{\lambda}^{*}\right)$, which is exactly like (CP ${ }_{\lambda}^{*}$ ) except for the added constraint that the $\lambda$-abstract in question must be cumulatively stratified. Unfortunately, however, this is too much of a generalization in our present context, since the $\lambda$-abstract $[\lambda x(\exists G)(x$ $=G \& \sim G(x))$ ] involved in Russell's paradox is easily seen to be cumulatively stratified. That is, $\left(\operatorname{CSCP}_{\lambda}^{*}\right)$ is an insufficient restriction of $\left(\mathrm{CP}_{\lambda}^{*}\right)$ since it still leads to Russell's paradox.

Now although $[\lambda x(\exists G)(x=G \& \sim G(x))]$ is cumulatively stratified, it is not heterogeneously stratified, and therefore it will not fulfill the conditions for the (heterogeneously) stratified comprehension principle, (SCP $\left.{ }_{\lambda}^{*}\right)$, which is exactly like (CP $\left.{ }_{\lambda}^{\prime \prime}\right)$ except for the constraint that the $\lambda$-abstract in question must be (heterogeneously) stratified. This principle, it should be 
observed, is in accordance both with the simple theory of types briefly described in Appendix B of [POM] and with Frege's hierarchy of unsaturated concepts. That is, although unsaturated concepts cannot be cumulatively stratified, higher level relational concepts can be inhomogencously stratified. Such higher level relations are referred to by Frege as unequal leveled relations. Thus, for example, the second level relation of an object to a concept under which that object falls is said by Frege to be an unequal second level relation because it has as arguments both a saturated object and an unsaturated first level concept ([Gg], vol. 1, section 22). Needless to say, but (SCP ${ }_{\hat{\lambda}}$ ) posits a first level relation corresponding to this unequal second level relation of subsumption; that is,

$$
\left(\exists R^{2}\right)([\lambda x y(\exists G)(x=G \& G(y))]=R)
$$

is an instance of (SCP full accordance with Frege's double correlation thesis.

Unfortunately, however, precisely because predication stands for a relation according to (SCP $\left.\mathrm{S}_{\mathrm{\lambda}}\right)$, then being impredicable with respect to this relation is also specifiable in terms of (SCP $\left.{ }^{*}\right)$. That is, since $[\lambda x y(\exists G)(x=$ $G \& G(y))]$ is heterogeneously stratified, then so is $[\lambda z \sim[\lambda x y(\exists G)(x=G$ $\& G(y))](z, z)]$; and therefore despite its complexity of expression in terms of $\lambda$-abstracts, Russell's paradox of the concept that is predicable of itself if, and only if, it is not predicable of itself is derivable on the basis of (SCP' $)$ after all. ${ }^{1}$ It follows, accordingly, that if Frege's double correlation thesis is to apply to all higher level unsaturated relations, then we must assume that no such relations are inhomogeneously stratified, i.e., that there are no unequal higher level relations. In particular, we must not assume that there can be such an unequal second level relation as the subsumption of an object under a concept; for it is fundamental to Russell's paradox that predication cannot stand for a relation between an object and a concept-correlate.

\section{The Theory of Homogeneous Simple Types as a Second Order Logic}

If unsaturated higher level concepts, including relations, are not to be inhomogeneously stratified, then the appropriate restriction of $(\mathrm{CP})$, 
needless to say, is the homogeneously stratified comprehension principle, (HSCP that the $\lambda$-abstract in question must be homogeneously stratified. Indeed, in the second order logic of nominalized predicates in question, which hereafter we shall refer to as $\lambda \mathrm{HST}^{*}$, the only $\lambda$-abstracts recognized as well-formed are those that are homogeneously stratified. This does not mean, it should be noted, that every wff of $\lambda \mathrm{HST}^{*}$ must be homogeneously stratified, but only that the $\lambda$-abstracts occurring in such wffs are. That is, in general, where $\mathrm{n}$ is a natural number, a meaningful expression of type $\mathrm{n}$ (as defined in section 7, "Russell's Paradox Revisited") is a meaningful expression of type $\mathrm{n}$ in $\lambda \mathrm{HST}^{*}$ if, and only if, every $\lambda$-abstract occurring in that expression is homogeneously stratified.

It follows, accordingly, that if $F$ is a 1-place predicate variable (or constant), then $F(F)$ and $\sim F(F)$ are both wffs of $\lambda \mathrm{HST}^{*}$; and similarly if $[\lambda x \phi]$ is homogeneously stratified, then both $[\lambda x \phi]([\lambda x \phi])$ and its negation are well-formed formulas of $\lambda \mathrm{HST}^{*}$. In other words, the laws of logic, and the principle of excluded middle in particular, apply in $\lambda \mathrm{HST}^{*}$ to concept-correlates as the denotata of nominalized predicates no less so than they apply to objects or individuals in general; and in that regard, therefore, concept-correlates may be said to be objects or individuals "in the full sense" as far as $\lambda \mathrm{HST}^{*}$ is concerned.

The basic laws of logic according to $\lambda \mathrm{HST}^{*}$, it should be emphasized, are exactly those of standard second order predicate logic with identity already described in section 3, but extended now to include homogeneously stratified $\lambda$-abstracts and nominalized predicates. In other words, by an axiom of $\lambda \mathrm{HST}^{*}$ we understand any wff of $\lambda \mathrm{HST}^{*}$ that is either tautologous or of one of the following forms:

$$
\begin{array}{ll}
(\forall u)[\phi \rightarrow \psi] \rightarrow[(\forall u) \phi \rightarrow(\forall u) \psi], & \text { where } u \text { is an } \\
& \text { individual or } \\
& \text { predicate vari- } \\
& \text { able, }
\end{array}
$$

$(\mathrm{A} 2 *) \quad \phi \rightarrow(\forall u) \phi$,

where $u$ is an individual or predicate variable not occurring free in $\phi$, 
96 Frege, Russell, and Logicism

$\left(\mathrm{A}^{*}\right) \quad(\exists x)(a=x)$,

$\left(\mathrm{LL}^{*}\right) \quad(a=b) \rightarrow[\phi \leftrightarrow \psi]$,

$\left(\operatorname{Id}{ }^{*}\right)$

(HSCP $\left.{ }^{*}\right)$ $\left(\exists F^{\mathrm{n}}\right)\left(\left[\lambda x_{1} \ldots x_{11} \phi\right]=F^{\mathrm{n}}\right)$, where $a$ is a singular term of $\lambda \mathrm{HST}^{*}$ in which $x$ does not occur free, where $a, b$ are singular terms of $\lambda \mathrm{HST}^{*}$ and $\psi$ comes from $\phi$ by replacing one or more free occurrences of $b$ by free occurrences of $a$,

$\left(\lambda\right.$-Conv") $\quad\left[\lambda x_{1} \ldots x_{n} \phi\right]\left(a_{1}, \ldots, a_{n}\right)$ $\leftrightarrow \phi\left(a_{1} / x_{1}, \ldots, a_{n} / x_{n}\right)$,

where $a_{1}, \ldots, a_{\mathrm{n}}$ are singular terms of $\lambda \mathrm{HST}^{*}$ and each $a_{\mathrm{n}}$ is free for $x_{n}$ in $\phi$, where $P$ is an n-place predicate variable or constant, where $F^{n}$ does not occur free in $\phi$ and $\left[\lambda x_{1}\right.$ $\left.\ldots x_{n} \phi\right]$ is homogeneously stratified.

Modus ponens and universal generalization (of an individual or predicate variable) are still the only inference rules, and theoremhood and derivability from premises are defined in the usual way. ${ }^{2}$

Now it is important to note that if $\phi$ is a $\lambda$-free wff containing no 
nominalized occurrences of predicates, then $\left[\lambda x_{1} \ldots x_{11} \phi\right]$ is homogeneously stratified, and therefore by (HSCP $(\mathrm{CP})$ of standard second order predicate logic with identity is easily seen to be provable in $\lambda \mathrm{HST}^{*}$. In other words, any $\lambda$-free wff containing no nominalized occurrences of predicates that is a theorem of standard second order predicate logic with identity is a theorem of $\lambda \mathrm{HST}^{* *}$ as well. In this regard, $\lambda \mathrm{HST}^{*}$ goes beyond the laws of standard second order predicate logic only in its recognition of homogeneously stratified $\lambda$-abstracts as complex predicates and in its logistic treatment of nominalized predicates, whether simple or complex, as singular terms. Both of these features are incorporated in the comprehension principle ( $\left.\mathrm{HSCP}_{\bar{\lambda}}\right)$, which, as already indicated, is in full accordance with Frege's double correlation thesis, so long as we assume that unsaturated higher level concepts are only homogeneously stratified.

In regard to the question of the consistency of $\lambda \mathrm{HST}^{*}$, let us note first that the full $\lambda \mathrm{HST}^{*}$ system can be shown to be consistent relative to monadic $\lambda \mathrm{HST}^{*}$ (i.e., $\lambda \mathrm{HST}^{*}$ restricted to monadic predicates only) in essentially the same way that the simple theory of types can be shown to be consistent relative to the simple monadic theory of types. That is, it can be shown that if monadic $\lambda \mathrm{HST}^{*}$ is consistent, then the full $\lambda \mathrm{HST}^{*}$ system is also consistent (cf. [NBC-4], section 4.8). Secondly, by interpreting monadic predication as membership, monadic $\lambda \mathrm{HST}^{*}$ can be readily shown to be consistent relative to R. Jensen's system NFU ("New Foundations with Urelements"); and therefore if NFU is consistent, then so is the full $\lambda$ HST $^{*}$ system. In [Jensen], however, Jensen has shown that NFU is consistent relative to weak Zermelo set theory ${ }^{3}$; and therefore, by putting these results together, we can make the following consistency claim regarding the full $\lambda \mathrm{HST}^{*}$ system (cf. [NBC-4], section 4.9).

THEOREM: If weak Zermelo set theory is consistent, then so is $\lambda$ HST"* $^{*}$.

\section{Frege and the Principle of Extensionality}

We observed in section 7 that (LL") and $(\lambda$-Conv") together yield the following generalized form of Frege's basic law $(\mathrm{Vb})$ :

$$
\left[\lambda x_{1} \ldots x_{n} \phi\right]=\left[\lambda x_{1} \ldots x_{n} \psi\right] \rightarrow\left(\forall x_{1}\right) \ldots\left(\forall x_{n}\right)(\phi \leftrightarrow \psi) .
$$


It was this law, it will be remembered, that together with $\left(\mathrm{A} 3^{*}\right)$ and the unrestricted comprehension principle $\left(\mathrm{CP}_{\lambda}^{*}\right)$ led to Russell's paradox. Given the restriction of $\left(\mathrm{CP}_{\lambda}^{*}\right)$ to (HSCP $\left.{ }_{\lambda}^{*}\right)$, however, Russell's paradox is no longer derivable (if weak Zermelo set theory is consistent), and we are still able to maintain Frege's basic law (Vb) as a law of logic.

Of course, this is only one direction of Frege's basic law V. The other direction, i.e. Frege's basic law ( $\mathrm{Va})$, is the following principle of extensionality:

$$
\left(\forall x_{1}\right) \ldots\left(\forall x_{n}\right)(\phi \leftrightarrow \psi) \rightarrow\left[\lambda x_{1} \ldots x_{n} \phi\right]=\left[\lambda x_{1} \ldots x_{n} \psi\right] .
$$

This principle, needless to say, is not provable in $\lambda \mathrm{HST}^{*}$, and in that regard, it might be claimed, it is not a "law of logic." For Frege, however, it is a law of logic because concepts and relations are (unsaturated) functions from objects to truth-values. That is, according to Frege, "what two concept-words mean [bedeuten] is the same if and only if the extensions of the corresponding concepts coincide" ([PW], 122). In making this claim, Frege is aware that he has made "an important concession to the extensionalist logicians. They are right," he claims, "when they show by their preference for the extension, as against the intension, of a concept that they regard the meaning [Bedeutung] and not the sense of words as the essential thing for logic. The intensionalist logicians are only too happy not to go beyond the sense; for what they call the intension, if it is not an idea, is nothing other than the sense. They forget that logic is not concerned with how thoughts, regardless of truth-value, follow from thoughts, that the step from thought to truth-value-more generally, the step from sense to meaning has to be taken in. They forget that the laws of logic are first and foremost laws in the realm of meanings $[\mathrm{Be}$ deutungen] and only relate indirectly to sense" (ibid.). In other words, as far as the laws of logic are concerned, "concepts differ only so far as their extensions are different" (ibid., 118); and "therefore just as proper names can replace one another salva veritate, so too can concept-words, if their extension is the same" (ibid.).

Regardless of his commitment to the principle of extensionality as a law of logic, however, it must not be overlooked here that Frege still maintains "that the concept is logically prior to its extension" ([G\&B], 106), and that he regards "as futile the attempt to take the extension of a concept as a class, and make it rest, not on the concept, but on single things" 
(ibid.). In other words, despite his commitment to the principle of extensionality, Frege's second order logic of value-ranges as conceptcorrelates is not a second order set theory in the sense in which sets are essentially constituted or composed of their members. That this is so is especially brought out by the reconstruction and identification of Frege's form of logicism with the system $\lambda \mathrm{HST}^{*}+\left(\mathrm{Ext}^{*}\right)$ as a second order logic of nominalized predicates. This system, we might note, is not only consistent relative to Jensen's "set" theory NFU, but is in fact equiconsistent with NFU. (Cf. chapter 4, section 6.)

\section{Russell and the Principle of Rigidity}

The only fundamental difference between Frege's and Russell's early form of logicism so far emphasized is that for Russell concepts are their own concept-correlates. That is, Russell refused to accept the unsaturated nature of concepts, and he assumed accordingly that nominalized predicates denote the same concepts that those predicates otherwise stand for in their role as predicates. The predicative nature of a concept for Russell, in other words, does not consist in its being an unsaturated function from objects to truthvalues, but rather only in its being a function whose values are propositions. That is, concepts for Russell are none other than propositional functions, and propositional functions are themselves individuals.

Now as functions whose values are propositions rather than truthvalues, propositional functions are intensional and not extensional entities; and this in fact is another fundamental difference between Frege's and Russell's early form of logicism. For when applied to $\lambda \mathrm{HST}^{*}$, this difference is reflected in the acceptance of (Ext $\left.{ }^{*}\right)$ by Frege as a law of logic as opposed to its rejection as such by Russell. That is, besides assuming that concepts have an individual nature, Russell also assumed that in general they are only intensionally individuated.

What exactly Russell means by the intensionality of a proposition, and thereby of a propositional function as well, he never says. Nevertheless, in our reconstruction of his early form of logicism, we shall assume that propositions can be represented by (or rather correlated with) functions from possible worlds to truth-values. This is not what a proposition really is according to Russell, needless to say, especially since possible worlds would themselves be constructed in Russell's early framework in 
terms of propositions about merely possible as well as actual individuals ${ }^{4}$; but it does serve as an intuitive guide regarding the intensional individuation of propositional functions. Thus, instead of Frege's principle of extensionality, (Ext"), being a law of logic, Russell (or so we shall assume) would have as a law of logic the corresponding principle of intensionality:

$\left(\square \mathrm{Ext}^{*}\right) \quad \square\left(\forall x_{1}\right) \ldots\left(\forall x_{\mathrm{n}}\right)(\phi \leftrightarrow \psi) \rightarrow\left[\lambda x_{1} \ldots x_{\mathrm{n}} \phi\right]=\left[\lambda x_{1} \ldots x_{\mathrm{n}} \psi\right]$.

This means that we shall need to take $\square$ as a new primitive logical constant of our logical grammar, and that we shall need to add the following clause in the definition of a meaningful expression given in section 7:

$$
\text { if } \phi \in \mathrm{ME}_{1} \text {, then } \square \phi \in \mathrm{ME}_{1} \text {. }
$$

We shall retain all of the axioms and inference rules of $\lambda \mathrm{HST}{ }^{*}$, except that now these axioms and rules are understood to apply to wffs containing occurrences of $\square$ as well. In addition, we shall also assume the axiom schemas of the $\mathrm{S} 5$ modal propositional logic and the rule of modal generalization; i.e., the rule that if $\phi$ is provable, then so is $\square \phi$. For convenience, we shall refer to the resulting system as $\square \lambda \mathrm{HST}^{*}$. Our initial proposal, accordingly, is that Russell's early form of logicism is to be reconstructed as $\square \lambda \mathrm{HST}^{*}+\left(\square \mathrm{Ext}{ }^{*}\right) .^{5}$

Unfortunately, however, $\square \lambda H S T^{*}+\left(\square \mathrm{Ext}^{*}\right)$ does not suffice for Russell's view of classes as analyzable in terms of concepts. In particular, assuming that classes are to be represented in terms of concepts (as individuals), we shall need an account of how this representation is to be given; and assuming the adequacy of that account we shall need to establish the following thesis as one of its consequences:

$$
(\forall F)(\exists G)(C l s(G) \&(\forall x)[F(x) \leftrightarrow G(x)]) .
$$

Now without going into the details here, we shall only say that Russell's own later contextual analysis in $[P M]$ of ' $\mathrm{Cls}$ ', or of expressions for particular classes as "incomplete symbols," will not suffice in our present context, since as reconstructed here, Russell's contextual analysis would have the inappropriate consequence that all concepts are "classes"; i.e., that concepts are extensionally, not intensionally, individuated after all. Instead of Russell's analysis, however, we can begin with the notion of a rigid ( $\mathrm{n}$-ary) propositional function as a concept that has the same exten- 
sion in every possible world. A class, on this analysis, will then simply be a rigid concept. Indeed, if concepts are themselves individuals, as Russell claimed, there would be little or no point in distinguishing a rigid concept from the class that is the extension of that concept, at least not if classes are really "superfluous" in Russell's form of logicism. ${ }^{6}$ Accordingly, where definitions of predicate constants are given in terms of homogeneously stratified $\lambda$-abstracts, we define the notion of rigidity ${ }_{\mathrm{n}}$ as follows:

$$
\begin{aligned}
& \text { Rigid }_{\mathrm{n}}={ }_{\mathrm{df}}\left[\lambda x ( \exists F ^ { \mathrm { n } } ) \left(x=F^{\mathrm{n}} \&\left(\forall y_{1}\right) \ldots\left(\forall y_{\mathrm{n}}\right)\left[\square F\left(y_{1}, \ldots, y_{\mathrm{n}}\right)\right.\right.\right. \\
& \left.\left.\left.\mathrm{v} \square \sim F\left(y_{1}, \ldots, y_{\mathrm{n}}\right)\right]\right)\right] .
\end{aligned}
$$

Thus, an n-ary relation-in-extension, on this analysis, is simply a rigid ${ }_{n}$ relation (in intension), and a class is simply a rigid ${ }_{1}$ concept:

$$
C l s={ }_{\mathrm{df}}\left[\lambda x\left(\exists F^{1}\right)\left(x=F \& \operatorname{Rigid}_{1}(F)\right)\right] .
$$

Now the fundamental new assumption that we need as a "law of logic" in Russell's intensional form of logicism is not just the above thesis that every concept is extensionally equivalent to a rigid concept (i.e., that the extension of a concept exists), but rather the following more general principle of rigidity:

$$
\begin{aligned}
\left(\forall F^{n}\right)\left(\exists G^{\mathrm{n}}\right)\left(\operatorname{Rigid}_{\mathrm{n}}(G)\right. & \&\left(\forall x_{1}\right) \ldots\left(\forall x_{n}\right)\left[F\left(x_{1}, \ldots, x_{n}\right)\right. \\
& \left.\left.\leftrightarrow G\left(x_{1}, \ldots, x_{n}\right)\right]\right) .
\end{aligned}
$$

Our proposal, accordingly, is that Russell's early form of logicism is to be reconstructed as the system $\square \lambda \mathrm{HST}^{*}+\left(\square \mathrm{Ext}{ }^{*}\right)+(\mathrm{PR})$.

It is clear, of course, that by interpreting $\square$ as double negation, $\square \lambda \mathrm{HST}$ * $+\left(\square \mathrm{Ext}^{* *}\right)+(\mathrm{PR})$ collapses to just $\lambda \mathrm{HS} \mathrm{T}^{* *}+\left(\mathrm{Ext}^{*}\right)$. That is, on our reconstruction, Russell's (early) intensional form of logicism is equiconsistent with Frege's extensional form; and therefore by the equiconsistency of the latter with Jensen's "set" theory NFU, we have the following result (cf. [NBC-4], section 6.3).

THEOREM: $\square \lambda$ HST $^{*}+(\square \mathrm{Ext} *)+(\mathrm{PR})$ is equiconsistent with both $\lambda \mathrm{HST}^{*}$ $+($ Ext" $)$ and Jensen's "set" theory NFU.

Classical mathematics, it is clear, is reducible to our reconstruction of Russell's intensional form of logicism no less so than it is to our reconstruction of Frege's extensional form. The identification of NFU- "sets" 
with Frege's value-ranges as concept-correlates is perhaps more plausible than their identification with rigid concepts; but then their identification with concepts to begin with should also more readily obviate their confusion with sets as essentially constituted or composed of their members.

\section{Frege's Contemplation of the Abelardian View}

In reconstructing Frege's logicism, it should be emphasized, we have made only one relatively minor change in his overall view. We have assumed, in particular, that all higher level unsaturated concepts are only homogeneously stratified; i.e., that there are no unequal higher level relations. Only on the basis of this assumption, in other words, can we maintain that $\left(\mathrm{CP}^{*}\right)$ is to be restricted to (HSCP $\left.{ }^{*}\right)$ in accordance with Frege's double correlation thesis.

There is an alternative to this assumption, however, that allows us to retain both $\left(\mathrm{CP}_{\lambda}^{*}\right)$ and to avoid Russell's paradox. This alternative, moreover, is implicit in Frege's discussion of Russell's paradox and is contained in his suggestion that we might "suppose there are cases where an unexceptional concept has no class answering to it as its extension" ([G\&B], 235). In other words, instead of maintaining for each singular term the dubious existential presupposition that that singular term actually denotes (a value of the bound individual variables), we are to allow

on this suggestion that some singular terms, and in particular some nominalized predicates, are denotationless.

Given the standard second order predicate logic with nominalized predicates described in section 7-that is, the logistic context in which Russell's paradox was originally formulated-the proposal in question, accordingly, amounts to replacing the dubious axiom (A3"), which explicitly expresses all such existential presuppositions, by the following weaker, but also unexceptionable, law of logic:

$$
\left(\mathrm{A} 3^{* *}\right) \quad(\forall x)(\exists y)(x=y) \text {. }
$$

A little more is actually needed on this proposal, however, since without $\left(\mathrm{A} 3^{*}\right)$ the identity wff $(a=a)$, also an unexceptionable law of logic, is no longer derivable. In replacing ( $\left.\mathrm{A} 3^{*}\right)$ by $\left(\mathrm{A} 3^{* *}\right)$, in other words, we shall also need to add $(a=a)$ as an axiom, where $a$ is any singular term. Similarly, in dropping all existential presuppositions regarding singular terms, 
we shall need to replace $\left(\lambda\right.$-Conv $\left.{ }^{*}\right)$ by the presupposition free form of $\lambda$ conversion:

$$
\begin{aligned}
\left(\exists / \lambda-\text { Conv }^{*}\right) \quad & {\left[\lambda x_{1} \ldots x_{\mathrm{n}} \phi\right]\left(a_{1}, \ldots, a_{\mathrm{n}}\right) \leftrightarrow\left(\exists x_{1}\right) \ldots\left(\exists x_{\mathrm{n}}\right) } \\
& \left(a_{1}=x_{1} \& \ldots \& a_{\mathrm{n}}=x_{\mathrm{n}} \& \phi\right),
\end{aligned}
$$

where no $x_{\mathrm{i}}$ is free in any $a_{\mathrm{j}}$, for all $\mathrm{i}, \mathrm{j}$ such that $1 \leq \mathrm{i}, \mathrm{j} \leq \mathrm{n}$.

Now with the replacement of (A3") by $\left(\mathrm{A} 3^{* *}\right)$ and $(a=a)$, and of $(\lambda-$ Conv") by $\left(\exists / \lambda\right.$ Conv $\left.^{*}\right)$, the principle of universal instantiation (UI*) regarding singular terms is no longer provable, it turns out, except in the following qualified form:

$$
(\exists y)(a=y) \rightarrow[(\forall x) \phi \rightarrow \phi(a / x)],
$$

where $a$ is any singular term that is free for $x$ in $\phi$ and in which $y$ has no free occurrences. With this qualification, however, what follows from Russell's paradox as described in section 7 is not a contradiction but only that the complex predicate $[\lambda x(\exists G)(x=G \& \sim G(x))]$ is denotationless in its occurrences as a singular term; i.e., instead of a contradiction,

$$
\sim(\exists y)([\lambda x(\exists G)(x=G \& \sim G(x))]=y)
$$

is provable. In other words, even though (CP unsaturated concept corresponding to $[\lambda x(\exists G)(x=G \& \sim G(x))]$ as a predicate, nevertheless by Russell's argument it is provable that there can be no saturated object corresponding to $[\lambda x(\exists G)(x=G \& \sim G(x))]$ as a singular term; or in Frege's words here is a case "where an unexceptional concept has no class answering to it as its extension" (ibid.).

Unfortunately, however, mathematics, and arithmetic in particular, is not reducible to the system resulting from the above changes; for unlike the situation in $\lambda \mathrm{HST}^{*}$ where (A3*) remains in force, but where only $\lambda$ abstracts that are homogeneously stratified are recognized as well-formed (and where $\left(\mathrm{CP}_{\lambda}^{*}\right)$, accordingly, is replaced by $\left(\mathrm{HSCP}_{\lambda}^{*}\right)$ ), we can no longer prove that there are any objects denoted by nominalized predicates, or, equivalently, that there are any concept-correlates at all. (The logical grammar of the system in question, it should be emphasized, is just the full unrestricted grammar described in section 7 , and the axioms are $\left(\mathrm{A} 1^{*}\right),\left(\mathrm{A} 2^{*}\right),\left(\mathrm{A} 3^{* *}\right),(a=a),\left(\mathrm{LL}^{*}\right),\left(\mathrm{Id}_{\lambda}^{*}\right),\left(\exists / \lambda-\mathrm{Conv}^{*}\right)$ and $\left.\left(\mathrm{CP} \mathrm{\lambda}^{*}\right).\right)$

That there might be no concept-correlates at all, incidentally, was not a possibility Frege refused to consider in his discussion of Russell's para- 
dox. For in commenting on the proposal in question, Frege seemed to interpret this alternative as one in which we can only "regard class names as sham proper names, which would thus not really have any reference" (ibid., 236). We agree, it should be noted, that for Frege this apparently meant that "class" names "would have to be regarded as part of signs that had reference only as wholes" (ibid.), as, e.g., names of natural numbers might occur as parts of quantifier expressions, but in our present framework this can also be formulated as the Abelardian thesis that no concept is a thing (cf. [NBC-4], section 4.1):

$$
\left(\forall F^{\mathfrak{n}}\right) \sim(\exists x)\left(F^{\mathfrak{n}}=x\right) .
$$

In other words, instead of maintaining only that some nominalized predicates must be denotationless, Frege generalized this alternative into the Abelardian view that all nominalized occurrences of predicates are denotationless. Abelard, incidentally, apparently believed that the same universal can be shared by different individuals, but he refused to grant that what individuals have in common is a "thing"; i.e., he denied that universals are individuals. In this regard, he might be said to have anticipated Frege's view of concepts as unsaturated functions. Be that as it may, nevertheless it was Frege's view throughout most of his career that nominalized predicates, and abstract singular terms in general, denoted objects even though those objects could not themselves be unsaturated concepts. For Abelard, on the other hand, at least on our interpretation of his view here, nominalized predicates were singular terms that simply failed to refer to any individual at all. Such a view, needless to say, is obviously "safe" from Russell's paradox, and indeed the result of adding the Abelardian thesis to the system in question can be shown to be consistent (cf. [NBC-4], section 4.10).

Though consistent, however, such a view does not lead to a reconstruction of Frege's form of logicism; and in fact, it leads directly away from it, a direction that Frege was not disinclined to take in 1924-25 at the end of his long and (from our present vantage point) brilliant career in defense of logicism. Thus, in speaking of "the formation of a proper name after the pattern of 'the extension of the concept $a$ ' " ([PW], 269), Frege not only noted that "because of the definite article, this expression appears to designate an object" (ibid.), but he went on to suggest that "there is no object for which this phrase could be a linguistically appropriate des- 
ignation" (ibid.). We are misled by language here, he suggests, and "from this has arisen the paradoxes of set theory which have dealt the death blow to set theory itself" (ibid.).

These final thoughts of a great logician are unfortunate, however, for the paradoxes, and Russell's paradox in particular, do not affect set theory as a theory of classes that are composed of their members, but rather affect only a theory of classes as the extensions of concepts; and whereas set theory has continued to flourish throughout the twentieth century, it is the theory of classes as the extensions of concepts, and thereby logicism itself, that has fallen into disrepute. Such disrepute is not deserved, however, since logicism, and Frege's form of logicism in particular, can be easily reconstructed in such a way as to avoid the paradoxes. In this regard, we have not only the reconstruction of Frege's logicism already given, but the following alternative reconstruction as well.

\section{A Second Reconstruction of Frege's Logicism}

The proof that some nominalized predicates must be denotationless in the system presently under consideration, it should be noted, does not show that all must be denotationless; i.e., the Abelardian thesis is not provable in our modified second order logic with nominalized predicates as described above in section 13. We may assume, in other words, that some concepts do have concept-correlates after all.

Such an assumption, needless to say, can be given in a number of different ways, such as those corresponding to the existence conditions for sets in different set theories. The latter, however, will result only in a second order theory of sets in the sense in which sets are essentially constituted or composed of their members; and dropping the axiom of extensionality so as to avoid calling such objects sets is really pointless, since their existence conditions are in accordance with the limitation of size doctrine, which in turn is based on the notion of sets as composed of their members. Such an assumption, in other words, will not result in a coherent form of logicism since as objects that are essentially constituted or composed of their members sets are mathematical and not logical objects. The relevant assumption, in this regard, must be based on Frege's double correlation thesis and the way it pertains to the positing of concept-correlates as logical ob- 
jects, and not on conditions that pertain to the existence of sets as composed of their members.

Now in returning to Frege's double correlation thesis in our present framework where $\lambda$-abstracts need not be homogeneously stratified, we should note that there is a difference in applying the thesis in the positing of first level concepts from applying it in the positing of concept-correlates. In particular, since the full unrestricted comprehension principle, $\left(\mathrm{CP}_{\lambda}^{*}\right)$, is to remain as an axiom schema, we in effect retain the unrestricted form of Frege's double correlation thesis as it applies to the positing of first level concepts; i.e., the form in which it posits first level concepts corresponding to unequal leveled or inhomogeneous higher level relations no less so than to homogeneous or equal leveled relations. Thus, corresponding to Frege's unequal second level relation of subsumption or predication, there is a first level relation posited by $\left(\mathrm{CP}_{\mathrm{\lambda}}^{*}\right)$; i.e.,

$$
\left(\exists R^{2}\right)([\lambda x y(\exists G)(x=G \& G(y))]=R)
$$

is provable in the system in question. Of course, by Russell's argument, this same predicate when nominalized simply fails to denote (a value of the bound individual variables). In other words, as applied to conceptcorrelates, Frege's double correlation thesis must be restricted; and in particular it is not to apply in general to inhomogeneous higher level relations. The assumption we shall make here is that it is to apply at least to all higher level unsaturated concepts that are homogeneously stratified; i.e., to all of the concepts that have concept-correlates in $\lambda \mathrm{HS}^{*}$.

In order to formulate our assumption as an axiom schema, we shall say that a meaningful expression $\xi$ (as defined in terms of the logical grammar of section 7) is bound to individuals if, and only if, for all natural numbers n, all n-place predicate variables $F^{\mathrm{n}}$, and all $\phi$, if $\left(\forall F^{\mathrm{n}}\right) \phi$ is a wff occurring in $\xi$, then for some individual variable $x$ and some wff $\psi, \phi$ is the wff $\left[(\exists x)\left(F^{\mathrm{n}}=x\right) \rightarrow \psi\right]$. In other words, to be bound to individuals, every predicate quantifier occurring in $\xi$ must refer only to those concepts posited by $\left(\mathrm{CP}_{\lambda}^{\prime \prime}\right)$ that have corresponding concept-correlates. Our assumption may now be stated as the following axiom schema:

$\left(\exists / \mathrm{HSCP}_{\lambda}^{*}\right) \quad(\exists y)\left(a_{1}=y\right) \& \ldots \&(\exists y)\left(\mathrm{a}_{\mathrm{k}}=y\right) \rightarrow(\exists y)\left(\left[\lambda x_{1} \ldots x_{\mathrm{n}} \phi\right]=\right.$ $y)$, 
where $\left[\lambda x_{1} \ldots x_{\mathrm{n}} \phi\right]$ is homogeneously stratified, $\phi$ is bound to individuals, $y$ is an individual variable not occurring in $\phi$, and $a_{1}, \ldots, a_{\mathrm{k}}$ are all of the variables or non-logical constants occurring free in $\left[\lambda x_{1} \ldots x_{n} \phi\right]$.

The axiom schemas of our present system, accordingly, are (A1*), $\left(\mathrm{A} 2^{*}\right),\left(\mathrm{A} 3^{* *}\right),(a=a)$, where $a$ is any singular term, $\left(\mathrm{LL}^{*}\right),\left(\mathrm{Id}_{\lambda}^{*}\right),(\exists / \lambda-$ Conv"), ( $\left.{ }^{*}{ }_{\lambda}^{*}\right)$ and now $\left(\exists /\right.$ HSCP $\left._{\lambda}^{*}\right)$ as well. ${ }^{7}$ (Modus ponens and universal generalization are its only inference rules.) Because of its relation to our earlier system, we shall refer to this system hereafter as HST* ${ }_{\lambda}^{*}$.

We must again emphasize that unlike the situation in $\lambda \mathrm{HST}^{*}, \lambda$ abstracts are not required to be homogeneously stratified in HST ${ }_{\lambda}^{*}$. That is, the meaningful expressions of HST ${ }_{\lambda}^{*}$ are just those described in the logical grammar of section 7. Also, unlike $\lambda \mathrm{HST}^{*}$, the system HST" is free of existential presuppositions regarding singular terms, including of course nominalized predicates as abstract singular terms. It follows, accordingly, that $\mathrm{HST}_{\lambda}^{*}$ is not a conservative extension of $\lambda \mathrm{HST}^{*}$, since whereas, by (A3"), $\left(\forall F^{\prime \prime}\right)(\exists x)(F=x)$ is provable in $\lambda \mathrm{HST}^{*}$, this same wff is actually disprovable in HST ${ }^{*}$. Nevertheless, since every wff of $\lambda \mathrm{HST}^{*}$ is provably equivalent, again by (A3*), to a wff that is bound to individuals, HST* may be said to contain $\lambda \mathrm{HST}^{*}$ in the sense of the following lemma (which is easily seen to hold).

LEMMA: If $\phi$ is a wff of $\lambda \mathrm{HST}^{*}$ that is bound to individuals, $y$ is an individual variable not occurring in $\phi$, and $a_{1}, \ldots, a_{\mathrm{k}}$ are all the variables or non-

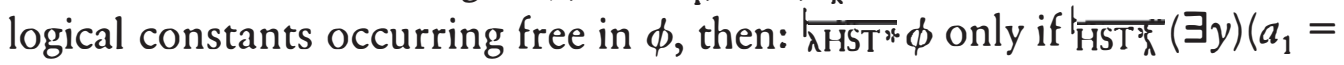
y) \& . \& \& $(\exists y)\left(a_{\mathrm{k}}=y\right) \rightarrow \phi$.

Restricting ourselves to pure wffs, i.e., wffs in which no predicate or individual constants occur, it follows by the above lemma that every sentence (or wff with no free variables) of $\lambda \mathrm{HST}^{*}$ that is bound to individuals and provable in $\lambda \mathrm{HST}^{*}$ is therefore provable in HST ${ }^{*}$ as well. In addition, by $\left(\exists / \mathrm{HSCP}_{\lambda}^{*}\right)$, every object that is a concept-correlate in $\lambda \mathrm{HST}^{*}$ is also a concept-correlate in HST ${ }_{\lambda}^{*}$; and therefore, for the same reason, every object that is a concept-correlate in $\lambda \mathrm{HST}^{*}+\left(\mathrm{Ext}^{*}\right)$ is also a conceptcorrelate in HST" $+\left(\right.$ Ext $\left.^{*}\right)$. Accordingly, since Jensen's "set" theory NFU is contained in $\lambda \mathrm{HST}^{*}+\left(\mathrm{Ext}^{*}\right)$, then in the sense of the above lemma NFU is also contained in HST ${ }^{*}+\left(\right.$ Ext $\left.^{*}\right)$; and in that regard classical mathematics, and arithmetic in particular, is reducible to HST ${ }_{\lambda}^{*}+$ 
$\left(\mathrm{Ext}^{*}\right)$ at least to the same extent that it is reducible to NFU. (Actually, $\mathrm{HST}_{\lambda}^{*}+\left(\mathrm{Ext}^{*}\right)$ is an improvement over $\lambda \mathrm{HST}^{*}+\left(\mathrm{Ext}^{*}\right)$, and therefore over NFU as well, for the same reason that Quine's "set" theory ML is an improvement over NF. In particular, in both HST* $+\left(\mathrm{Ext}^{*}\right)$ and ML, mathematical induction is provable in an unrestricted form (cf. [Quine], section 42).) Our proposal that HST ${ }_{\lambda}^{*}+\left(\right.$ Ext $\left.^{*}\right)$ be taken as an alternative, and perhaps a preferred, reconstruction of Frege's form of logicism is, in that case, quite in order.

Finally, it should be noted that just as Hao Wang was able to prove the consistency of Quine's set theory ML relative to NF, we are able to prove the consistency of HST $T_{\lambda}^{*}+\left(\right.$ Ext $\left.^{*}\right)$ relative to $\lambda \mathrm{HST}^{*}+\left(\mathrm{Ext}^{*}\right)$, and therefore relative to NFU as well (cf. chapter 4 , section 7 and [NBC-4], section 6.4).

THEOREM: If $\lambda \mathrm{HST}^{*}+\left(\mathrm{Ext}^{*}\right)$ is consistent, then so is HST $\mathrm{H}^{*}+\left(\mathrm{Ext}^{*}\right)$.

\section{An Intensionalized Form of Frege's Logicism}

By the intensional counterpart of HST $\mathrm{T}_{\lambda}^{*}$ we shall understand the system HST $_{\lambda}^{*} \square$ that is developed from HST ${ }_{\lambda}^{*}$ in the same way that $\square \lambda \mathrm{HST}^{*}$ was developed from $\lambda \mathrm{HST}^{*}$. That is, the axiom schemas of HST ${ }_{\lambda}^{*} \square$ are just those of HST $T_{\lambda}^{*}$, extended now to apply to wffs containing $\square$ as well, plus those of the S5 modal propositional logic. The inference rules are of course the same as those for $\square \lambda \mathrm{HST}^{*}$, viz., modus ponens, universal generalization and modal generalization.

Now it is perhaps noteworthy that insofar as $\left(\square \mathrm{Ext}{ }^{*}\right)$ is to be assumed as well, then we need not take $\square$ as a primitive logical constant since it can in that case be contextually defined as follows:

$$
\square \phi={ }_{\mathrm{df}}[\lambda \phi]=[\lambda(\forall x)(x=x)] .
$$

That is, since the biconditional,

$$
\square \phi \leftrightarrow[\lambda \phi]=[\lambda(\forall x)(x=x)]
$$

is provable in HST ${ }_{\lambda}^{*}+\left(\square \mathrm{Ext}^{* *}\right)$, then we need not take $\square$ as a primitive logical constant of this system since it can be introduced by means of the above contextual definition. (Some, but not all, of the modal axioms will then become redundant.) This definition is not available in $\square \lambda \mathrm{HST}^{*}+$ 
$\left(\square E x t^{*}\right)$, our reconstruction of Russell's early form of logicism, since it cannot be used in that context to explain occurrences of $\square$ in wffs that are not homogeneously stratified. That is, the above definiens, as a wff of $\lambda$ HST $^{*}$, would restrict the application of $\square$ to wffs that are homogeneously stratified, since only those wffs can occur within the $\lambda$-abstracts of $\lambda \mathrm{HST}^{*}$. But since there are wffs of $\lambda \mathrm{HST}^{*}$, including tautologous wffs, that are not homogeneously stratified, defining $\square$ as above would in that case fail to validate the rule of modal generalization when applied to those axioms of $\lambda \mathrm{HST}$ " that are not homogeneously stratified. It is only in HST ${ }_{\lambda}^{*}$, in other words, where $\lambda$-abstracts are not required to be homogeneously stratified that the above definition of $\square$ will suffice.

In addition to the principle of intensionality, ( $\square \mathrm{Ext} *$ ), we shall also need to assume the principle of rigidity, (PR), if we are to represent classes in this alternative the way they are represented in $\square \lambda H S T^{*}+$ $(\square \mathrm{Ext} *)+(\mathrm{PR})$, our reconstruction of Russell's early form of logicism. Of course, with the addition of (PR) we can bring about the same reduction of classical mathematics to logic as is already achieved in HST" which contains all NFU-"sets" as concept-correlates. Moreover, we can prove the relative consistency of HST" the same way as we proved the relative consistency of HST $+\left(\mathrm{Ext}^{*}\right)$.

THEOREM: If $\square \lambda$ HST $^{*}+\left(\square \mathrm{Ext}^{*}\right)+(\mathrm{PR})$ is consistent, then so is HST ${ }^{*} \square+$ $\left(\square \mathrm{Ext}^{*}\right)+(\mathrm{PR})$.

Despite its relative consistency, however, HST"* $+\left(\square \mathrm{Ext}^{*}\right)+(\mathrm{PR})$ cannot be construed as a reconstruction of Russell's early form of logicism. For as an abstract singular term a nominalized predicate, according to Russell, denotes the same concept as an individual that the predicate in question otherwise stands for in its role as a predicate; and therefore whatever is the value of a bound predicate variable, according to early Russell, is also a value of the bound individual variables. That is, in Russell's early form of logicism no predicate can stand for a concept in its role as a predicate and yet fail to denote an individual when transformed into an abstract singular term. But since there are predicate expressions

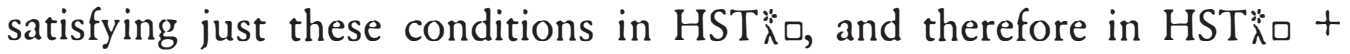
$\left(\square \mathrm{Ext}{ }^{*}\right)+(\mathrm{PR})$ as well, then neither HST" $\mathrm{x}$ nor any of its extensions can be the basis of a reconstruction of Russell's early form of logicism. In 
other words, unlike Frege's form of logicism, we have no way in Russell's framework by which to explain why some concepts have individuals as concept-correlates while others do not, since on Russell's account concepts are their own concept-correlates.

We might adopt a mixed strategy here, however, whereby concepts are unsaturated functions in Frege's sense but still have propositions as values in Russell's sense. Concepts are then not individuals after all, and there is nothing odd or contradictory in the idea of some concepts having a concept-correlate while others do not. Of course, if in addition conceptcorrelates are none other than the extensions that these concepts have in different possible worlds, then the resulting framework may be described more as an intensionalized form of Frege's logicism than as a counterpart to Russell's. In this regard, we would not adopt ( $\square$ Ext") but (Ext*) instead, since even intensional concepts that are materially equivalent will have the same extension, and therefore concepts that are materially equivalent in a given possible world will have the same concept-correlate in that world. In such a framework, needless to say, the principle of rigidity, (PR), would be completely superfluous.

There is a problem here with HST"艹a as the basis for such an intensionalized form of Frege's logicism, however, since by (LL*),

$$
\left(\forall F^{\mathrm{n}}\right)\left(\forall G^{\mathrm{n}}\right)(F=G \rightarrow \square F=G)
$$

is provable in HST*̈, and therefore all concepts, even when intensionalized, will always have the same extension. We can of course modify (LL*) so that it remains valid only for extensional contexts, i.e., only for wffs in which $\square$ does not occur; for in that case concepts having the same extension in one possible world need not necessarily have the same extension in every possible world. That is, in that case,

$$
\left(\exists F^{n}\right)\left(\exists G^{n}\right)(F=G \& \diamond F \neq G)
$$

will be consistent and not inconsistent (cf. [NBC-4], section 6.5). Thus, if HST $x^{*}$ is the system resulting from HST ${ }^{\circ}$ by weakening (LL") in this way, but also by adding the commutative law,

$$
\left(\forall F^{n}\right) \square \phi \leftrightarrow \square\left(\forall F^{n}\right) \phi,
$$

as an axiom schema (since concepts themselves remain the "same" from world to world), then we can identify this intensionalized form of Frege's 
logicism with HST落 $+\left(\right.$ Ext $\left.^{*}\right)$. The extensional system HST⿳亠丷⿵冂丶 $+\left(\mathrm{Ext}^{*}\right)$ described earlier, needless to say, is a subsystem of HST** by interpreting $\square$ as double negation the two systems collapse into one, thereby showing the consistency of HST落 $+\left(\mathrm{Ext}^{*}\right)$ relative to HST + $\left(\mathrm{Ext}^{*}\right)$.

\section{Russell's Logicism as Conceptual Platonism}

The mixed strategy described above favors Frege's form of logicism over Russell's, it should be noted, since unsaturated concepts, even when intensionalized, were assigned their extensions as their concept-correlates in the different possible worlds. There is another mixed strategy, however, that is more in line with Russell's form of logicism than with Frege's, and in fact it may be taken as a counterpart even to Russell's later form of logicism in $[P M]$.

On this mixed strategy, the predicative nature of a concept consists in its having both an intensional and an unsaturated nature, though the latter is understood in even a more radical sense than Frege would have allowed. In particular, on this account all predicable concepts are cognitive capacities to identify (in a classificatory sense), characterize or relate objects in various ways, and the purely dispositional or nonoccurrent nature of such a capacity is none other than its unsaturated nature. That is, on this account, concepts are intersubjectively realizable cognitive abilities that may be exercised by the same person at different times as well as by different persons at the same time; and in that regard concepts are neither ideas nor mental images in the sense of particular mental occurrences. The excrcise (or saturation) of a concept on this account does indeed result in a mental event, i.e., a mental act, and, if overtly expressed, a speech act as well; but the concept is not itself the mental or speech act as an event but rather what accounts for that act's predicable nature. Second level concepts are really referential concepts on this account, incidentally, and it is their joint exercise or mutual saturation with predicable concepts (in a kind of mental chemistry) that is the basis of our particular thoughts and acts of communication (cf. [NBC-4], chap. 2, and my "Philosophical Perspectives on Quantification in Tense and Modal Logic," ([NBC-3]), sections 11-15).

Predicable concepts, on this version of conceptualism, are not indepen- 


\section{Frege, Russell, and Logicism}

dently real properties or relations, it must be emphasized; and, unlike referential concepts, their primary role in thought and communication is not referential but predicative. Yet, by a curious development of the interplay between language and thought, predicable concepts as cognitive capacities can be transformed into secondary or derived abilities that enable us to apply predicable concepts in a denotative manner corresponding to the use of nominalized predicates in natural language. It is by means of such a secondary or derived application, moreover, that we purport to refer to independently real platonic forms as the denotata of nominalized predicates. Thus, for example, not only do we predicate of a shape that it is triangular or of a person that he is wise by applying a predicable concept in each case, but, in addition, we also purport to denote the properties of triangularity and wisdom, respectively, by applying these same concepts denotatively.

Purporting to denote and actually denoting are of course not the same, and in fact, despite all our purportings, there may be no independently real properties or relations at all that are actually denoted by any nominalized predicates; or at least that is a view that is compatible with our present account of concepts as unsaturated cognitive capacities. Adopting the Abelardian thesis here, however, will not result in a counterpart to Russell's form of logicism. In other words, we shall consider here only the alternative platonic view according to which most, even if not all, of the predicable concepts that we can form and articulate actually do denote an independently real property or relation when applied denotatively. That some predicable concepts that we can form and articulate must fail to denote such a property or relation will, on such a view, be an interesting but hardly problematic fact. What would be problematic, of course, is the different platonic view that concepts are themselves the independently real properties and relations they purport to denote when applied denotatively while admitting that only some of them are individuals while others are not.

Now it might be objected that if concepts are cognitive capacities, then none of them can be impredicatively formed in the Russellian sense in which they can be specified only by means of a wff that contains a quantifier ranging over all concepts. In other words, as based on the capacities of the human intellect, the laws of compositionality regarding concept-formation in the sense intended here exclude the possibility of 
validating the full impredicative comprehension principle $\left(\mathrm{CP}_{*}^{*}\right)$. In this regard, only a conceptualism that is "constructive" in a sense corresponding to the theory of ramified types will be permitted.

While such a constructive conceptualism can be formalized as a logic of nominalized predicates without resorting to Russell's treatment of concepts as "improper objects" for which the law of excluded middle is to be restricted, we shall, for reasons of space, forego such a presentation here; for our concern is with the validation, not the invalidation, of $\left(\mathrm{CP}_{\hat{\lambda}}^{*}\right){ }^{8}$ In this regard, it should be noted, there is a form of conceptualism, which we shall call bolistic conceptualism, that agrees that the initial, and perhaps most important, stages in concept-formation are "constructive" in the above sense, but which nevertheless maintains that there is also a stage of concept-formation (usually occurring only in postadolescence) at which so-called impredicative concept-formation becomes possible. Such a stage is realized, moreover, only through our capacity for language, and in particular through our capacity to use language for the expression of "constructible" concepts. Impredicative concept-formation, in other words, is a mediated process, and language and the linguistic ability to use predicate expressions is the means used to master and direct such a process. Holistic conceptualism, in this regard, presupposes constructive conceptualism as an antecedent stage of conceptual development, and in particular as a stage that is subsequently reconstructed through a certain reiterable pattern of reflective abstraction that proceeds through the ramified hierarchy of predicable concepts and that is finally completed or closed only by an idealized transition to a limit whereby impredicative concept-formation becomes possible (cf. [NBC-4], chap. 2, and my "Conceptualism, Ramified Logic, and Nominalized Predicates" ([NBC2]), sections 3 and 7).

According to holistic conceptualism, in other words, the impredicative comprehension principle (CP) of standard second order predicate logic is valid as a description of the laws of compositionality for conceptformation, as long as the predicate quantifiers occurring therein are taken as ranging over all predicable concepts, including those that are only impredicatively specifiable. A holistic conceptualism that also adopts the platonic assumption described above regarding the denotative application of predicable concepts will in that case also validate ( $\left.\mathrm{CP}_{\hat{\lambda}}^{*}\right)$ as well. In other words, HST ${ }^{\prime} \square$ can serve as the basis for a formulation of Concep- 
tual Platonism as described above. Moreover, if thoughts are assumed to be at least in principle always overtly expressible in language, and $\square$ is interpreted as ranging over all possible contexts of use of language, i.e., in the sense of pragmatics, then the principle of intensionality, ( $\square \mathrm{Ext} \mathrm{t}^{*}$ ), as a principle regarding the conditions under which predicable concepts are understood to denote the same property or relation when applied denotatively, might also be assumed as valid in Conceptual Platonism. (Nothing essential really depends on this assumption, however; i.e. ( $\square \mathrm{Ext}^{*}$ ) can be dropped as a basic law of logic in Conceptual Platonism.)

HST ${ }^{*} \square+\left(\square \mathrm{Ext}^{* *}\right)$ does not itself suffice for the reduction of classical mathematics to logic, however; and in particular the axiom of infinity is not provable in HST" $+\left(\square \mathrm{Ext}^{*}\right)$, just as it is not provable in either $\mathrm{HST}_{\lambda}^{*}+\left(\mathrm{Ext}^{*}\right)$ or HST* ${ }^{* *}+\left(\mathrm{Ext}^{*}\right)$ as well. That there are infinitely many properties and relations as platonic forms is no doubt plausible, if we are assuming that there are platonic forms as individuals to begin with; but it is not in any case a consequence of Conceptual Platonism in the sense in which the latter assumes only that predicable concepts denote properties or relations when applied denotatively. In this regard, Conceptual Platonism, as well as each of our earlier reconstructions, is somewhat like Russell's later form of logicism in $[P M]$ in that an axiom of infinity is not provable in that framework as well. In $[P M]$, however, the axiom of infinity requires that there be infinitely many concrete individuals, and that assumption is less plausible than one regarding properties and relations as abstract individuals. In particular, an axiom of infinity of the form:

$$
\left(\forall F^{n}\right)\left(\forall G^{\mathrm{k}}\right)(F \neq G) \text {, where } \mathrm{n} \neq \mathrm{k},
$$

is especially natural, since it only assumes that different properties or relations are denoted by predicable concepts of different degrees (adicity).

The situation is more problematic for an account of classes as the extensions of concepts in HST ${ }^{*} \square+\left(\square \mathrm{Ext}^{*}\right)$, however, though such an account is forthcoming if we also assume the principle of rigidity, (PR). But (PR), unfortunately, cannot be validated in Conceptual Platonism, since as a principle about concepts as cognitive capacities it amounts to an unwarranted (and in fact contraindicated) reducibility axiom to the effect that every predicable concept is materially equivalent to one having the same extension (or rigid property or relation when applied denotatively) in ev- 
ery possible context of use. (PR), in other words, can be validated only by the full platonic view that identifies concepts as being themselves the independently existing properties and relations they otherwise purport to represent, which is to say that (PR) can be validated only in Russell's early form of logicism reconstructed as $\square \lambda \mathrm{HST}^{*}+\left(\square \mathrm{Ext}{ }^{*}\right)+(\mathrm{PR})$.

Russell's own reducibility axiom in his theory of ramified types is also said to be unwarranted (and contraindicated) in his later form of logicism, incidentally. If it is, then this can only be so because the concepts or propositional functions assumed in the theory of ramified types are not independently real properties and relations, as Russell in fact described them to be, but are rather really concepts as cognitive capacities in the sense intended here. In that case, however, they cannot also be the logical objects in terms of which classical mathematics is to be explained, since as capacities they are not objects at all. Russell's later form of logicism, in other words, is better understood as a form of Conceptual Platonism, though apparently one based only on constructive rather than holistic conceptualism as well.

The upshot is that although HST" $+\left(\square \mathrm{Ext}^{*}\right)+\left(\mathrm{Inf}^{*}\right)+(\mathrm{PR})$, can be taken as a counterpart to Russell's later form of logicism, nevertheless neither HST ${ }^{*} \square+\left(\square E x t^{*}\right)+\left(\right.$ Inf $\left.^{*}\right)$ nor Russell's theory of ramified types suffices as an adequate framework for logicism. This still leaves our reconstruction of Russell's early form of logicism intact, however, and it in no way affects either of our reconstructions of Frege's form of logicism. In other words, as philosophical doctrines go, logicism, whether in Frege's or Russell's early form, is alive and well.

\section{Notes}

1 I am indebted to Edmund Gettier for bringing this fact about (SCP*) to my attention.

2 The rewrite law

$$
\left[\lambda x_{1} \ldots x_{n} \phi\right]=\left[\lambda y_{1} \ldots y_{n} \phi\left(y_{1} / x_{1}, \ldots, y_{n} / x_{n}\right)\right],
$$

where no $y_{i}$ occurs in $\phi$, is derivable in $\lambda \mathrm{HST}^{*}$ on the basis of the principle of extensionality, $\left(\mathrm{Ext}^{*}\right)$, i.e., Frege's basic law $(\mathrm{Va})$, or its intensional counterpart, $\left(\triangle E x t^{*}\right)$, described in sections 11 and 12 below. If neither of these principles are assumed, then the rewrite law must be taken as an additional axiom schema of $\lambda \mathrm{HST}^{*}$. 
3 Weak Zermelo set theory is the restriction of Zermelo set theory to those instances of the Aussonderungsaxiom in which all quantifiers in the comprehension clause are limited or restricted; i.e. in which all quantifiers have the form $(\forall x \epsilon y) \phi$ or $(\exists x \in y) \phi$.

4 Cf. chapter 3 for a description of Russell's early framework as a form of possibilism.

5 Note that by (LL*),

$$
(a=b) \rightarrow \square(a=b)
$$

is provable in $\square \lambda \mathrm{HST}^{*}+\left(\square \mathrm{Ext} \mathrm{t}^{*}\right)$, and therefore so are

$$
(\exists x) \square(a=x)
$$

and

$$
(\exists F) \square\left(\left[\lambda x_{1} \ldots x_{\mathrm{n}} \phi\right]=F\right),
$$

where $x$ does not occur free in $a$, and $F^{n}$ does not occur free in $\left[\lambda x_{1} \ldots x_{n} \phi\right]$, and $\left[\lambda x_{1} \ldots x_{n} \phi\right]$ is homogeneously stratified. From these principles, the following commutative laws follow:

$$
\begin{aligned}
& (\forall x) \square \phi \leftrightarrow \square(\forall x) \phi \\
& \left(\forall F^{\prime \prime}\right) \square \phi \leftrightarrow \square\left(\forall F^{\prime \prime \prime}\right) \phi .
\end{aligned}
$$

As applied to concrete individuals, the first of these commutative laws makes sense only in Russell's early possibilist framework (cf. chapter 3 ).

6 This approach towards the representation of classes and relations-in-extension was first given by Montague in Formal Philosophy, ([FP]), 132. It also occurs in [Gallin], 77, where the principle of extensional comprehension is the type-theoretical counterpart of the principle of rigidity described below.

7 As indicated for $\lambda \mathrm{HST}^{*}$, the rewrite law,

$$
\left[\lambda x_{1} \ldots x_{n} \phi\right]=\left[\lambda y_{1} \ldots y_{n} \phi\left(y_{1} / x_{1}, \ldots, y_{n} / x_{n}\right)\right]
$$

where no $y_{i}$ occurs in $\phi$, is provable in this system on the basis of either $\left(\operatorname{Ext}^{*}\right)$ or $\left(\square \mathrm{Ext}^{*}\right)$. If neither of these principles are to be assumed, however, then the rewrite law must be added as an axiom schema.

8 A complete formalization of constructive conceptualism as a second order logic with nominalized predicates as abstract singular terms can be found in [NBC-2].

\section{References}

Frege, Gottlob

[Fd] The Foundations of Arithmetic, trans. by J. L. Austin, Harper \& Bros., N.Y. 1960.

[G\&B] Translations from the Philosophical Writings of Gottlob Frege, eds. P. Geach and M. Black, Blackwell, Oxford, 1952.

[Gg] Die Grundgesetze der Arithmetik, vols 1 and 2, Hildesheim, 1962. 
[PW] Posthumous Writings, eds. H. Hermes, F. Kambartel, F. Kaulbach; trans. by P. Long and R. White, Blackwell, Oxford 1979.

[PMC] Philosophical and Mathematical Correspondence, eds. G. Gabriel, H. Hermes, F. Kambartel, C. Thiel, A. Veraart; trans. by H. Kaal, University of Chicago Press, Chicago, 1980.

Russell, Bertrand

[AMR] An Analysis of Mathematical Reasoning, unpublished ms. of 1898, in the Bertrand Russell Archives, McMaster University, Hamilton, Ontario, Canada.

[POM] The Principles of Mathematics, 2nd ed., W. W. Norton \& Co., N.Y., 1937.

$[P M]$ Principia Mathematica, coauthor, A. N. Whitehead, Cambridge University Press, 1913.

[EA] Essays in Analysis, ed. D. Lackey, Braziller, N.Y., 1973.

Carnap, Rudolf

[Carnap] "The Logicist Foundations of Mathematics," Erkenntnis, 2 (1931); trans. by E. Putnam and G.J. Massey and reprinted in Philosophy of Mathematics, eds. H.

Church, Alonzo Putnam and P. Bennacerraf, Prentice Hall, 1964.

[Church] "Schröder's Anticipation of the Simple Theory of Types," The Journal of Unified Science (Erkenntnis) vol. 9 (1939): 149-52.

Cocchiarella, Nino

[NBC-1] "A Second Order Logic of Variable-Binding Operators," Reports on Mathematical Logic, vol. 5 (1975): 3-18.

[NBC-2] “Conceptualism, Ramified Logic, and Nominalized Predicates," Topoi 5 (1986): $75-87$.

[NBC-3] "Philosophical Perspectives on Quantification in Tense and Modal Logic," in Handbook of Philosophical Logic, vol. 2, eds. D. Gabbay and F. Guenthner, D. Reidel Pub. Co. Dordrecht, 1984, pp. 309-53.

[NBC-4] Logical Investigations of Predication Theory and the Problems of Universals, vol. 2 of Indices, Bibliopolis Press, Naples, 1986.

Gallin, Daniel

[Gallin] Intensional and Higher-Order Modal Logic, No. Holland, Amsterdam, 1975. Henkin, Leon

[Henkin] "Banishing the Rule of Substitution for Functional Variables," The Journal of Symbolic Logic, vol. 18 (1953): 201-8.

Jensen, Ronald

[Jensen] "On the Consistency of a Slight(?) Modification of Quine's New Foundations," Synthesé, vol. 19 (1968): 250-63.

Kalish, Donald and Montague, Richard

[K\&M] "On Tarksi's Formalization of Predicate Logic with Identity," Arch. für Math. Logik und Grundl., 7 (1965): 61-79.

Montague, Richard

[FP] Formal Philosophy, ed. R. H. Thomason, Yale University Press, New Haven, 1974. 


\section{Frege, Russell, and Logicism}

Quine, Willard Van Orman

[Quine] Set Theory and its Logic, Harvard University Press, Cambridge, 1963.

Tarski, Alfred

[Tarski] "Der Wahrheitsbegriff in Formalisierten Sprachen," Studia Philosophica, vol. 1 (1936):261-405. 


\section{Meinong Reconstructed versus Early Russell Reconstructed}

Contemporary philosophy is in a rut, according to Terence Parsons in his recent book Nonexistent Objects, ([NO]), and it is one that stems from the (post-1905) work of Bertrand Russell. The main characteristic of this "Russellian rut" ([NO],1) is strict adherence to the thesis that being, or being something, amounts to being something that exists-or equivalently that 'there is' is to be equated with 'there exists' ([NO], 6). This view is now so well entrenched, according to Parsons, that it is a main stay of what he also calls the orthodox tradition.

Now the orthodox view is in a rut, according to Parsons, "because it's a view in which most of us are so entrenched that it's hard to see over the edges" ([NO], 1). Naturally, if we want "to look over the edge and see how things might be different" ([NO], 8), as any objective seeker of truth would, then "we need to encounter an actual theory about nonexistent objects" (ibid.). It is the construction and presentation of such a theory that is Parsons's concern in Nonexistent Objects.

\section{Meinongian Objects versus Russellian Individuals}

Parsons's approach to the topic of nonexistence is through Meinong's theory and notion of a "concrete" object, i.e., the notion of a nonideal or nonabstract Meinongian object. (Concreteness in this context, it should 
be noted, does not imply existence-or even possible existence, according to Parsons and Meinong.) Curiously, Parsons gives no recognition or other mention to Russell's own earlier (pre-1905) theory in [POM], which is also committed to nonexisting concrete objects-though not at all in the same sense of 'object' as intended by Meinong, and now by Parsons as well. This is surprising because one of Parsons's goals in this text is to make the topic of nonexistence understandable to those who approach it from within the orthodox tradition, i.e., the tradition that stems from Russell after 1905 (cf. [NO], 9). With that goal in mind, however, would not the best approach be to develop Russell's own earlier theory rather than Meinong's-i.e., the theory from which the orthodox tradition arose once nonexisting concrete individuals were excluded? And, in fact, has not the development of several variants of such a Russellian theory already been carried out (even if not intentionally as a reconstruction of Russell's early views) in the form of higher order intensional or modal logics based on Russellian individuals that need not exist in the actual world but which do exist in some possible world or other? Indeed, in this regard might not the essential difference between a pre-1905 and a post-1905 Russellian theory be described in terms of the current actualism-possibilism debate, i.e., the debate whether quantification over concrete individuals should be with respect to all possible concrete individuals or only with respect to those existing in the world in question? (Russell himself eschewed talk about possible worlds, to be sure; but he would have allowed, at least in $[P O M]$, some such equivalent talk about maximal classes of propositions having possible individuals as constituents, i.e., propositions that, though not all true, could have all been true.)

Now we do not object to Parsons's choice of Meinong's theory here, nor for that matter to his elegant reconstruction and presentation of that theory. We do think, however, that a more balanced recognition of Russell's overall view is called for and that perhaps the best way to make the Meinongian notion of a concrete object understandable to the orthodox tradition is to compare it with the general Russellian notion of a concrete individual, i.e., the Russellian notion of an individual that can exist but which might in fact not exist. Indeed, on the basis of the analysis and comparison we shall give here, it is our position that the Meinongian notion of a concrete object, at least as reconstructed by Parsons, is parasitic 
upon, though in a beneficent way, the Russellian notion of a concrete individual, existent or otherwise.

In the way of a succinct summary and brief reminder of Russell's early view, the following quote is probably the most appropriate: "there is only one kind of being, namely being simpliciter, and only one kind of existence, namely existence simpliciter" ([POM], 449). Explained more fully, "being is that which belongs to every conceivable term, to every possible object of thought-in short to everything that can possibly occur in any proposition, true or false, and to all such propositions themselves" (ibid.) (Russell, it will be remembered, uses 'term', 'entity', and 'unit' as synonymous with 'individual'-cf. [POM], 43.) Existence, on the other hand, is not the same as being, for existence "is the prerogative of some only amongst beings" (p. 449). That is, "to exist," according to this early Russell, "is to have a specific relation to existence-a relation which existence itself does not have" (ibid.), and which in fact no abstract individual can have. In other words, according to Russell, all and only concrete individuals can exist-which is not to say that abstract individuals, e.g., properties, relations and propositions cannot $b e$. Not all concrete individuals actually exist, on the other hand, which again is not to say that they do not have being. The distinction between existence and being "is essential", according to Russell, "if we are ever to deny the existence of anything. For what does not exist must be something, or it would be meaningless to deny its existence; and hence we need the concept of being, as that which belongs even to the non-existent" (ibid., 450, italics added). Being and being something, in other words, are the same for Russell, and it will not do to say that he was confused about the difference between being something and being something that exists.

This difference in fact was important for Russell even in "On Denoting" of 1905, [OD], where properties, relations, and propositions are still individuals (and values of the formal variable $x$ ), but not individuals that exist. (Russell's first theory of types in Appendix B of [POM] was not for properties, relations and propositions, but for classes-as-many. It was not until his later no classes theories of types, in 1908 and 1910, that properties, relations, and propositions were typed and 'individual' was reserved for concrete individuals.) The new assumption about individuals in [OD], according to Russell, was that "we are able to hold that there are no un- 


\section{Meinong versus Early Russell}

real individuals" (p. 118). That is, according to the post-1905 Russell, there are no concrete individuals that do not exist; and therefore being something (concrete) can be restricted to being something that exists (which is not the same as to say that they are synonymous since in that case no assumption would be needed at all).

Now we are not concerned with these historical differences in Russell's position here, where our concern is rather with comparing Russell's notion of a concrete object with Parsons's reconstruction of Meinong's theory of concrete objects. In this regard we shall for convenience hereafter (until section 6) use 'individual' to refer to concrete Russellian individuals, existent or otherwise (but as understood in contemporary Russellian theories), and 'object' to refer to concrete Meinongian objects (as understood in Parsons's reconstruction).

\section{Russellian versus Meinongian Definite Descriptions}

Now the question of a Russellian alternative to Parsons's Meinongian theory is particularly relevant when one considers Parsons's "evidence" or "data" for his theory and his discussion of Russell's arguments against Meinong (chap. 2). For the evidence that there are "a host of particular propositions which we believe and which seem to commit us to unreal objects" (p. 32) is precisely of the same sort that Russell considered when he held that there are nonexisting (concrete) individuals (cf. [POM], 43). We need not review Parsons's specific examples here, accordingly, since in fact the evidence is not such that they can be accounted for only in Meinongian terms. Indeed, we maintain, but shall not pursue the details here, that a more natural account of these examples can be given in Russellian terms; e.g., in terms of the higher order modal predicate logic $\lambda$ HST $^{*}$ described below in section 6 or in terms of Montague's intensional logic in "The Proper Treatment of Quantification in Ordinary English" [PTQ], both of which are based on Russellian individuals, existent and otherwise.

It is noteworthy, incidentally, that Montague adopts Russell's 1905 theory of definite descriptions (in his translation of English into his intensional logic) even while he rejects Russell's claim that "we are able to hold that there are no unreal individuals," i.e., the claim or new assumption about non-existing concreta that Russell thinks can be based on 
that theory. Adopting Russell's 1905 theory of definite descriptions, in other words, does not commit one to the orthodox view that quantification over (concrete) individuals must be restricted to existing individuals. Indeed, Russell himself implicitly acknowledged this in his formulation of the ontological proof of the existence of "the most perfect Being" ([OD], 117). (Cf. also Russell's footnoted remark that "the argument can be made to prove validly that all members of the class of most perfect Beings exist," and, furthermore, "that this class cannot have more than one member." Of course, according to Russell, "taking the definition of perfection as possession of all positive predicates, it can be proved almost equally formally that the class does not have even one member" [ibid.].)

It is also noteworthy, on the other hand, and perhaps ironic as well, that Russell's earlier theory of denoting phrases (cf. [POM], chap. 5), though couched in a framework in which being something means being an individual, existent or otherwise, does not exclude his later assumption (now the keystone of the orthodox tradition) that being something (concrete) can be restricted to being something that exists. In particular, it is not part of this theory that every consistent denoting phrase will denote a possible individual that might have satisfied that phrase. Thus, where $a$ is a class-concept, Russell's "complete definition of a denoting concept which does not denote anything" ([POM], 74), though only a sufficient condition in the case of definite descriptions, is stated as follows: "the denoting concepts associated with $a$ will not denote anything when and only when ' $x$ is an $a$ ' is false for all values of $x$ " (ibid.). On the basis of this definition, Russell concludes, even while assuming that there are concrete individuals that do not exist, that the denoting phrase 'all chimeras' denotes nothing, since ' $x$ is a chimera' is false for all values of $x$, existent or otherwise. And in that regard it is clear that Russell also intends to claim that 'the gold mountain' denotes nothing, since ' $x$ is a gold mountain' is also false for all values of $x$, existent or otherwise (ibid.). In other words, it is one thing to actually be a chimera or a gold mountain, and quite another to be a possible individual that might have been a chimera or a gold mountain had the world been otherwise, i.e., had certain propo-! sitions that in fact are false been true.

For the Russell of [POM], accordingly, a denoting phrase does not in general denote any individual that might have satisfied that phrase, but only those individuals, existent or otherwise, that in fact do satisfy that 
phrase. In the special case of definite descriptions, moreover, it is clear that Russell intends such a phrase to denote an individual, existent or otherwise, if, and only if, that individual uniquely satisfies the phrase in question (cf. [POM], section 63). That is, though Russell himself does not engage in an explicit discussion on this matter in [POM], nevertheless it is implicit in his pre-1905 theory of definite descriptions (as unreduced singular terms) that the following schema is to be taken as valid:

$$
(\iota x) \varphi \text { is } P \equiv(\exists y)[(x)(\varphi \equiv x=y) \& y \text { is } P] .
$$

(That the validation of this schema requires a logic free of existential presuppositions regarding definite descriptions as singular terms, and that Russell himself had no such logic at hand may, incidentally, have been contributing, and perhaps even the decisive, factors in Russell's later abandoning this theory in 1905-especially since it too, after all, is consistent with the 1905 assumption that being something can be restricted to being something that exists.) Now the validation of (DD) in Russell's early theory is particularly significant here because it is this same schema (or rather its modal generalization) that Parsons takes as an axiom schema of his Meinongian theory (cf. [NO], 117)-though of course, whereas for Russell the quantifiers refer to individuals, existent or otherwise, for Parsons they are intended to refer to Meinongian objects. (Issues of scope in the substitution of wffs for the one-place predicate $P$ can of course be resolved either in terms of Russell's own later use of scope indicators or, as in Parsons's theory, in terms of the scope distinctions accompanying the use of the $\lambda$-operator for the representation, and substitution, of complex predicates.)

Parsons would of course be wrong to claim here (and he doesn't) that the above schema is representative of Meinong's theory. What he does claim, on the other hand, is that "the treatment given of definite descriptions [in this schema] is a fairly reasonable representation of English usage" ([NO], 118). If so, however, then since Parsons's principal if not sole source of "evidence" is English usage, it is clear that his evidence does not favor a Meinongian theory but a pre-1905 Russellian theory in- stead. In this regard, it might be noted that the Russell of [POM] would have agreed with Parsons's rejection of the theory given in [OD], even if being something (concrete) in the latter theory were not restricted to being something that exists. Thus, unlike the later Russell of [OD], the Rus- 
sell of $[P O M]$ denied that the proposition expressed by a sentence of the form 'All $A$ are $B$ ' is identical with the proposition expressed by a sentence of the form 'Every individual $x$ is such that if $x$ is $A$, then $x$ is $B$ '. The two propositions are "formally equivalent," to be sure, but they are not identical (cf. [POM], 74). The two propositions contain, in particular, different denoting concepts, viz., one corresponding to 'All $A$ ', and the other corresponding to 'Every individual'. Similarly, or so this early Russell would have claimed had he been more explicit on the matter, the proposition expressed by a sentence of the form "The individual $x$ such that $\varphi$ is $P$ ' is not identical with the proposition expressed by a sentence of the form "There is exactly one individual $x$ such that $\varphi$, and that individual is P'. The two propositions are indeed "formally equivalent," as already noted, which is why the schema (DD) is valid; but in this case, unlike the situation in [OD], it is not to be construed as a contextual definition of the definite description operator as an "incomplete symbol." That is, definite descriptions, according to early Russell, express denoting concepts that occur precisely as such in propositions.

Not all reconstructions of early Russell need agree in retaining definite descriptions as unreduced singular terms, of course; and, as already noted, Montague's treatment of definite descriptions in [PTQ] does not. In addition, neither interpretation, it should be noted, will really go beyond English usage. This is not the case with Parsons's presentation of his Meinongian theory, on the other hand, since "there is another usage which it [the scheme (DD)] doesn't capture" ([NO], 118), viz., "Meinong's usage when he speaks of 'the round square' or 'the gold mountain' " (ibid.); and it is Parsons's intention to represent this usage in his theory as well (though one might well wonder why this isn't "evidence" against his theory).

This is not to say that Parsons is willing to endorse what he calls the unrestricted satisfaction principle ([NO], 30); i.e., the assumption that any definite description refers to an object that satisfies that description. For of course that assumption is inconsistent, as Russell himself argued; and if this is indeed the purport of Meinong's use of 'the', then Meinong's original theory is also inconsistent. Be that as it may, it is clear in any case that Russell identifies Meinong's theory with this principle; and in that regard it is noteworthy that his-initial-objection-to-Meinong-s.theory is not that it posits individuals that do not exist but rather that it posits objects 
that do-not even subsist, such as the Meinongian objects that are the referents of 'the present King of France' and 'the round square' ([OD], 107).

Now we need not quibble here over Russell's misappropriated use of Meinong's notion of subsistence (Bestehen)-since, apparently, only ideal or abstract objects subsist according to Meinong, and the purported objects in question are not abstract but concrete. For by subsistence, it is clear, Russell means the same as being (cf. Russell, "Meinong's Theory of Complexes and Assumptions" [MTC], 36), i.e., the property that all individuals have, whether they be concrete or abstract, and whether they exist or not. In other words, it is because ' $x$ is a present King of France' and ' $x$ is a round square' are both false for all values of $x$, existent or otherwise, that Russell claims that the Menongian objects in question do not subsist.

Only one of the descriptions in question, it will be noted, is considered contradictory by Russell. The other is perfectly consistent and in some possible world it will, in fact, be uniquely satisfied by an individual that exists in that world. Such an individual, along with many others who will also uniquely satisfy that description in yet other possible worlds, will of course subsist in the real world, but neither it nor any of the others will actually satisfy that description in this world.

Parsons, incidentally, agrees that at least in the sense of 'the' that conforms with English usage, i.e., the sense of 'the' represented in the schema (DD), such a description as 'the present King of France' is improper and refers to nothing. But his agreement with early Russell on this fact is almost, as it were, purely coincidental. For according to Parsons, 'the present King of France' is improper not because ' $x$ is a present King of France' is false for all values of $x$, but rather because it is true for many values of $x$. Thus, e.g., in response to Quine's question whether the possible fat man in the doorway is identical with the possible bald man in the doorway (when in fact there exists no man in the doorway), Parsons states that "there are many possible fat men in the doorway" (p. 28), and similarly that there are many possible bald men in the doorway; and therefore the definite descriptions in question are improper and refer to nothing. For early Russell, on the other hand, at least as we interpret him here (and also the Russell of [OD] who in discussing Meinong's theory allows, at least for the sake of argument, that not all subsisting concrete individuals need exist), Quine's definite descriptions are improper not because there 
are many possible fat men and many possible bald men in the doorway, but rather because there are in fact no possible fat men and no possible bald men actually in the doorway; i.e., ' $x$ is a possible fat man in the doorway' and ' $x$ is a possible bald man in the doorway' are false for all values of $x$, existent or otherwise. (Of course 'possibly, $x$ is a fat man in the dourway' and 'possibly, $x$ is a bald man in the doorway' are true of many values of $x$, existent and otherwise.)

Russell's second and principal objection to Meinong's theory, or what he took to be Meinong's use of 'the', it will be remembered, is that Meinongian objects that do not subsist "are apt to infringe the law of contradiction" ([OD], 107). Thus, e.g., the existent present King of France, Russell claimed, both exists (by the unrestricted satisfaction principle) and does not exist (since ' $x$ is an existent present King of France' is false for all values of $x$, existent and otherwise, and therefore false for all values of $x$ that exist). Similarly, Russell claimed, "the round square is round, and also not round; etc." (ibid.). It is clear, of course, that as part of this last 'etc.' Russell intended to include such descriptions as 'the object that is golden and also such that it is not the case that it is golden', which Parsons himself gives as an example that shows that the unrestricted satisfaction principle is inconsistent. Yet Parsons seems to begrudge crediting Russell with this result and claims instead that "Russell's arguments against Meinong almost show this, though not quite" ([NO], 31).

Parsons's proposal for Meinong's use of 'the' is that it be represented by an additional description operator ' $m$ ' and interpreted in such a way that $(m x) \varphi$ is to "refer to the "minimum' of the objects which satisfy $\varphi$," and to the null object if there is no such minimum ([NO], 118). That is, "if some object satisfies $\varphi$, then $(m x) \varphi$ refers to the object whose nuclear properties are exactly those shared by all objects which satisfy $\varphi$, and if no object satisfies $\varphi$, then $(m x) \varphi$ refers to the null object" (ibid.).

The notion of a nuclear property that occurs in this proposal is a crucial part of Parsons's reconstruction of Meinong's theory, and it is intended to correspond to Meinong's distinction between properties that are konstitutorisch (nuclear) and those that are ausserkonstitutorisch (extranuclear). Thus, applied to Meinong's response regarding the existent present King of France, viz., that it is an object that is existent even though it does not exist, the distinction amounts to the claim that al- 
though existence is an extranuclear property that the existent present King of France does not have, nevertheless, being existent is a nuclear property that it does have. In this regard, the property of being existent is to be distinguished from the extranuclear property of existence by being its "watered down" nuclear version; and although such a nuclear version of an extranuclear property always and necessarily coincides with that extranuclear property as far as existing objects are concerned, they may nevertheless diverge in the case of nonexisting objects. Thus, even though the existent present King of France does not in fact have the "fullstrength" extranuclear property of existence, it nevertheless does have, apparently in virtue of the way it is presented, the "watered-down" nuclear version of this property, viz., the property of being an existent.

In regard to Russell's claim that the round square is both round and not round, Parsons strikes out on his own and takes the non-Meinongian line that although the round square is both round and square, it does not follow, as Russell claimed, that it is also not round; i.e., Parsons claims that being square does not exclude being round. Such a purported exclusion, Parsons argues, is not part of the meaning of 'square' ([NO], 39); and if it is a consequence of Euclidean geometry, then it is either part of an uninterpreted calculus, in which case it is not about the properties of being round and being square after all, or it is part of physical geometry, and therefore is at best empirical in content and not necessary after all (ibid.). Being square does not exclude being round, according to Parsons, since in fact, "not all square objects fail to be round" ([NO], 40), and "the round square provides an example of this" (ibid.).

The round square, according to Parsons, is not a contradictory object; and regardless of whether "Meinong may or may not have been willing to endorse contradictions" ([NO], 31), Parsons is not. However, while being square does not exclude being round for all Meinongian objects, Parsons does agree that "being square does exclude being round ... for all possible objects" ([NO], 40f). That is, while it is not a contradictory object, the round square is nevertheless an impossible object. How or why it is an impossible object, however, we are never told, and this is problematic since, according to Parsons, being square does not exclude being round. Indeed, one can only suspect that it is an impossible object because there is no Russellian (concrete) individual, existent or otherwise, to which it might correspond; i.e., because being square does exclude be- 
ing round after all, at least as far as Russellian individuals are concerned. (Cf. Parsons; "A Prolegomenon to Meinongian Semantics" [PMS], 567; and his "Nuclear and Extra-nuclear Properties, Meinong and Leibniz" [NEN], 141, for confirmation on this point). One begins to suspect, in other words, that even the notion of an impossible Meinongian object is parasitic upon the Russellian notion of a possible individual, i.e., of an individual that can exist even if it does not in fact exist.

Finally, although impossible Meinongian objects have no counterparts among Russellian individuals, nevertheless it is essential to Parsons's reconstruction of Meinong's theory that an impossible object not be, as it was for Meinong, a "homeless object," but rather that it be something; i.e., that formally it be construed as having being in the (pre-1905) Russellian sense of being the value of a bound variable. It is problematic, no doubt, how one might otherwise refer to Meinong's "homeless objects"; but still, their construal as something is in no way either justified or explained by noting the "evidential" difference between being something and being something that exists - and such a justification cannot be realized merely by chastising those caught in the (post-1905) "Russellian rut" for confusing the one with the other.

\section{An Orthodox "Picture" of Meinongian Objects}

Whether there really are impossible Meinongian objects or not, Parsons provides a model for the modal-free part of his theory and thereby proves it to be consistent. The model or "picture". that Parsons gives of Meinongian objects, moreover, is entirely within a modal-free Russellian higher order predicate logic where the individuals are all existing individuals and the properties and relations between individuals are those that can be possessed only by existing individuals. That is, the model is based upon what Parsons considers to be the orthodox tradition.

Now it should be noted that although nuclear properties and relations are identified by Parsons with the orthodox properties and relations of individuals, Meinongian objects are represented in the model by classes of properties of individuals. The possession of a nuclear property by a Meinongian object is in that case correlated with the membership of that property in the class of properties representing the object in question. It is significant, on the other hand, that extranuclear properties and relations 
are not also identified with orthodox properties and relations of individuals (there being none left with which to identify them), but with higher order properties and relations of classes of properties of individuals instead. The possession of an extranuclear property is in that case correlated with the orthodox Russellian predication of a higher order property or relation.

Such a difference in logical type and level in the representation of nuclear and extranuclear properties and relations, needless to say, might well be thought to be indicative of an underlying structural difference in Parsons's theory between two types or forms of predication, viz., a Meinongian predication of nuclear properties and relations, and a Russellian predication of extranuclear properties and relations. That is not the purport of Parsons's theory, however, and in any case it would still leave unexplained the real source of extranuclear properties and relations.

Though it is only a "picture" and, Parsons insists, not the intended interpretation, nevertheless Parsons's model does serve as an intuitive aid to understanding the structure of his theory, which hereafter we shall refer to as 0 . Thus, in particular, the model is easily seen to validate the two fundamental principles of $\mathcal{O}$; viz., (1) that no two Meinongian objects have exactly the same nuclear properties; and (2) that for any class of nuclear properties (or perhaps more accurately, for any condition of nuclear properties that can be specified in ( )), there is a Meinongian object that has all and only the nuclear properties in that class (cf. [NO], 19). Using Parsons's informal notation where nuclear predicates are indicated by a superscripted ' $N$ ' and extra-nuclear predicates by ' $E$ ', the first principle, also called the axiom of the identity of nuclear indiscernibles, can be formulated as follows:

$$
(x)(y)\left[\left(P^{N}\right)\left(P^{N} x \equiv p^{N} y\right) \supset x=y\right] .
$$

Parsons's second principle, also called the axiom for objects, can be formulated as the following schema:

$$
(\exists x)\left(P^{N}\right)\left(P^{N} x \equiv \varphi\right),
$$

where $\varphi$ is any wff of $\mathcal{O}$ in which $x$ does not occur free.

One immediate consequence of $(\mathrm{OBJ})$, where $\varphi$ is the wff $P^{N} \neq P^{N}$, is that there is a null object:

$$
(\exists x)\left(P^{N}\right) \sim P^{N} x
$$


which, by (INI), is unique. Similarly, where $\varphi$ is $P^{N}=P^{N}$, there is a full $o b$ ject:

$$
(\exists x)\left(P^{N}\right) P^{N} x
$$

which, again by (INI), is also unique. Of course, since every property has a "watered down" nuclear version (by a special "watering down" axiom), the full object has the "watered down" nuclear version of the complement of each nuclear property, as well as that property itself, and therefore the full object is an impossible Meinongian object; that is, it is not a possible object in the sense that it is impossible for there to exist an object that has every nuclear property that the full object has. Of course, on this (Parsons's) definition of a possible object, viz., that it is possible for there to exist an object that has every nuclear property that that object actually has (i.e. has in the actual world), the null object is a possible object (if any object is), since it is possible for there to exist an object that has all the nuclear properties that the null object actually has (i.e., has in the actual world).

Now it should be noted here that Parsons's modeling of Meinongian objects as classes of properties of existing individuals applies only to their representation in the actual world, though it is clear that the same type of representation is intended to apply to other worlds as well. That is, formodal purposes a Meinongian object would be modeled by a function from possible worlds to classes of properties of individuals existing in those worlds. But, as is clear from Parsons's semantics for his full theory, the "same" Meinongian object need not be represented by the same class of properties in every possible world (cf. clause (14), [NO], 130, of Parsons's definition of a semantic modal structure). That is, the nuclear properties a Meinongian object in fact has (in the actual world), it, i.e., the "same" object in the cross-world de re sense of identity, need not necessarily have (in other possible worlds). (Surprisingly, this important feature of Parsons's theory is hardly discussed by him even though it differs from his position in [NEN] where Meinongian objects were represented as having their nuclear properties essentially, i.e., as part of their essence or nature.) Thus, even though the full object, i.e., the referent of $(\iota x)\left(P^{N}\right) P^{N} x$, is impossible in the sense defined by Parsons, this does not mean that it, the "same" object (in the cross-world de re sense of identity), cannot exist. Indeed, the "same" object that is the full object in this 


\section{Meinong versus Early Russell}

world might be the null object in some other possible world; and in that regard what is possibly a possible object need not in fact be a possible object at all.

An object that is complete with respect to nuclear properties, i.e., which is such that for each nuclear property $P$, either the object has $P$ or it has the "watered down" nuclear version of the complement of $P$, is, by (INI), complete simpliciter. Of course, every object is complete with respect to extra-nuclear properties, and therefore every existing object is complete with respect to nuclear properties (since, by the special "watering down" axiom, an existing object has the "watered down" nuclear version of any extra-nuclear property it has, and therefore it either has any given nuclear property or it has the "watered down" nuclear version of the complement of that property). But not every complete object exists, on the other hand, nor need it even be a possible object-as witness the full object that is complete with respect nuclear properties, and therefore complete simpliciter.

An object that is both complete and possible with respect to its nuclear properties, Parsons calls a monad, and he suggests that this might in fact be taken as an explication of Leibniz's notion of a monad. However, since

$(\exists x)(x$ is possible $\& x$ is complete $\& \square \sim \mathrm{E} ! x)$

is consistent in 0 , even on the assumption that in every possible world something exists, it is clear that this could hardly be what Leibniz meant by a monad-or at least not if for Leibniz no monad can have the de re modal property of necessary nonexistence. On the other hand, requiring a Meinongian object to have the de re modal property of possible existence as well as being both complete and possible with respect to the nuclear properties it has (in the actual world) will also not do as an explication of a Leibnizian monad, since the nuclear properties such an object might have in any possible world in which it (the "same" object) exists could be completely different from those it has in the actual world and with respect to which it is complete and possible. Moreover, even an object that is complete and possible and has all its nuclear properties necessarily will not do, since such an object will either necessarily exist or necessarily not exist.

The notion of existence that occurs here, incidentally, is taken by Par- 
sons as a primitive extranuclear property; but other than the role it has in the "watering down" axiom and the "plugging up" of nuclear relations to make nuclear properties, no attempt is made to explain how it connects with the other nuclear properties that a Meinongian object might have. In particular, since every existing object might in some possible world be the full object, then e very existing object might be an impossible object. In this regard, existing Meinongian objects, at least as reconstructed by Parsons, cannot literally be the same as existing Russellian individuals, but can at best be correlated with them, and even then only in such a way that the same correlation need not carry over from one world to another. Meinongian objects, in other words, are really not "objects" in anything at all like the ordinary sense, but are rather something like "individual concepts" having classes of nuclear properties as their denotations in different possible worlds. Maybe Parsons's model or "picture" of Meinongian objects is really closer to the intended interpretation than he thinks.

\section{A Russellian "Picture" of the Nuclear/Extranuclear Distinction}

If Parsons's model or "picture" of Meinongian objects is about the best that can be done toward approximating the structure of the intended interpretation from within the strict orthodox tradition, then the major problem with this model or "picture" is its failure to provide an account of the nuclear/extranuclear distinction, and in particular of the real source of extra-nuclear properties and relations. Such an account is forthcoming, however, if we open the door of the orthodox view a little and let the pre-1905 Russellians do the "picturing" instead.

In this regard, the important distinction we shall make use of here from the pre-1905 Russellian point of view (and by which we shall explain the nuclear/extranuclear distinction) is that between properties and relations that entail existence, i.e., that cannot be possessed except by existing individuals, and those that do not, i.e., that can be possessed (in a given world) by nonexisting individuals as well. Russell, to be sure, did not himself explicitly discuss this distinction, but it does seem to be implicit in such claims of his as that 'all unicorns', 'all winged horses', 'all gold mountains', etc., denote nothing; i.e., that ' $x$ is a unicorn', ' $x$ is a winged horse', ' $x$ is a gold mountain', etc., are false for all values of $x$, existent or 
otherwise (cf. $[P O M], 74)$. In other words, at least as we interpret early Russell here, being a unicorn, being a winged horse (and indeed being winged simpliciter as well as being a horse simpliciter), and being a mountain, all of gold or otherwise, are properties that entail existence, i.e., that cannot be actually possessed except by existing individuals. Accordingly, since there exist no unicorns, winged horses, gold mountains, then there are (in the wider sense of being) no unicorns, winged horses, or gold mountains as well-which is not at all the same as to say that there are no possible unicorns, winged horses, gold mountains. Of course, unlike Parsons's notion of a Meinongian object that is a possible unicorn (winged horse, gold mountain), a Russellian individual is a.possible unicorn (winged horse, gold mountain) if, and only if, it is possible that it, the same.individual in the cross-world de re sense of identity, is a unicorn (winged horse, gold mountain).

In addition to these kinds of properties, however, (early) Russell is quite explicit in acknowledging that there are such properties as being an object of (someone's) thought and being worshipped (by someone) that do not entail existence and that are in fact possessed by some nonexisting (concrete) individuals. Moreover, if tense and temporal predication are taken seriously (as they are, e.g., in Montague's intensional logic as one representative of the pre-1905 Russellian view), then such temporal properties as being remembered (by someone), being a past King of France, and being an ancestor of everyone now existing, are also examples of properties that do not entail existence (at the times at which they are possessed), since they can be possessed by individuals at times when these individuals do not exist. Indeed, since any careful analysis of natural language predicates would show how sensitive they are in their temporal implications regarding the past, present, or future existence of the individuals to which they might be applied, it is clear that the distinction between properties and relations that entail existence and those that do not (or do not with respect to all of their argument "positions") is both implicit in natural language and deeply embedded in our common sense framework. (One example of the kind of temporal sensitivity we have in mind here, and given in my "Existence Entailing Attributes, Modes of Copulation and Modes of Being in Second Order Logic," [EEA], is the predicate for the ancestor relationship, the implications of which are that an individual $x$ is an ancestor of another individual $y$ only at those times 
when either $y$ exists and $x$ did exist, though it need not still exist, or when $x$ has continued to exist even though $y$ has ceased to exist. When $y$ no longer exists as well as $x$, we say that $x$ was an ancestor of $y$; and where $y$ has yet to exist, we say that $x$ will be an ancestor of $y$.)

Now we shall not take the distinction that is in question here to be exactly that between existence-entailing properties and relations, which hereafter we shall refer to as $e$-properties and $e$-relations, and properties and relations that definitely do not entail existence (with respect to each of their argument "positions"). Instead, we need only distinguish $e$-properties and $e$-relations from properties and relations in general, where the latter include those that entail existence as well as those that do not. Our proposal in that case will be that nuclear properties and relations are none other than $e$-properties and $e$-relations and that extranuclear properties and relations are properties and relations in general. (Those Parsons calls essentially extranuclear will of course be identified with those properties and relations of individuals that definitely do not entail existence.) Such as proposal, we maintain, will provide both a natural explanation of Parsons's artificial nuclear/extranuclear distinction and a natural source for extranuclear properties and relations. Of course, it must be understood that the explanation occurs within the context of "picturing" Meinongian objects as classes of $e$-properties (or, for modal considerations, as functions from possible worlds to classes of $e$-properties). For it is only in this way that we can explain why a Russellian individual that is round or square must then exist, whereas a Meinongian object that is round or square need not exist (in which case it can be both round and square) if we have somehow removed the "modal moment" of "full-strength factuality" from these properties (cf. [NO], 44).

In respect to an explicit representation for the sort of Russellian framework we have in mind here, we shall utilize the second order modal predicate logic formulated in my "A Completeness Theorem in Second Order Modal Logic," [ACT], where the distinction of $e$-properties and $e$-relations from properties and relations in general is taken as fundamental. (Cf. [EET] for an informal discussion of this logic.) The distinction can, of course, be constructed within Montague's intensional logic, but we prefer to take it as fundamental here, especially since Parsons's theory is also a second order modal predicate logic.

As indicated, accordingly, the Russellian logic in question is a second 
order modal predicate logic in which standard predicate quantifiers $\forall$ and $\exists$ are taken as referring to all properties, existence entailing and otherwise, when affixed to one-place predicate variables and to all $n$-ary relations, existence entailing and otherwise (with respect to any of their argument "positions"), when affixed to $n$-place predicate variables. In addition, there are special quantifiers, say $\forall^{i}$ and $\exists^{i}$, which when affixed to one-place predicate variables are taken as referring to all $\mathcal{c}$-properties, and which when affixed to $n$-place predicate variables refer to all $n$-ary $e$ relations (i.e., those relations that entail existence with respect to each of their argument "positions"). There is no distinction, it should be noted, between the sort of predicate variable that can be affixed to $\forall^{i}$ and $\exists^{i}$, as opposed to the sort that can be affixed to $\forall$ and $\exists$; i.e., for each positive integer $n$, there is but one sort of $n$-place predicate variable. (We shall take 0 -place predicate variables to range over propositions, but in that case there is no distinction between $\forall$ and $\forall^{e}$ or $\exists$ and $\exists^{e}$.) The overall logic, moreover, is understood to be that of standard second order modal predicate logic, i.e., standard second order predicate logic combined with an $\mathrm{S} 5$ modal propositional logic; and in that regard the logic of the restricted predicate quantifiers $\forall^{e}$ and $\exists^{e}$ amounts in effect to a "free" logic for $e$-properties and $e$-relations, i.e., a logic in which predicate expressions need not in general stand only for $e$-properties and $e$-relations.

With an existence predicate taken as primitive, needless to say, the special quantifiers $\forall^{c}$ and $\exists^{e}$ can be contexually defined as restricted forms of quantification over properties and relations in general, i.e., as restricted to those that entail existence (with respect to each of their argument "positions"). However, with $\forall^{e}$ and $\exists^{e}$ as primitive, existence can be analyzed, and therefore the existence predicate defined, as the possession of an $\mathcal{c}$ property. (Such an analysis would be especially appropriate, incidentally, if properties and relations were taken as the socio-biologically based concepts of a holistic conceptualism in which impredicative, as well as predicative, concept-formation is possible, since in that case the impredicatively formed concept of existence would be logically distinguished from ordinary predicative $e$-concepts in being holistically constructed or formed in terms of a totality to which it belongs. Meinongian objects would then be "pictured" in such a conceptualist framework by classes of predicative $e$ concepts rather than by classes of $e$-concepts in general. Though it is the author's own preferred framework, we have avoided it here in favor of its 
platonistic Russellian counterpart because, unlike the latter, it invalidates, rather than validates, Parsons's "watering down" axiom. Of course, for a conceptualist that may be reason enough to reject Parsons's Meinongian theory, at least to the extent that it resorts to a "watering down" axiom.)

It might be noted, incidentally, that within the Russellian logic in question existing individuals that are indiscernible in their $e$-properties are indiscernible simpliciter, and therefore are identical (whether identity be taken as a primitive or defined as indiscernibility with respect to all properties, existence entailing and otherwise). That is, the following is a provable thesis of the Russellian logic in question:

$$
(\forall x)(\forall y)\left(\mathrm{E} ! x \& \mathrm{E} ! y \supset\left[\left(\forall^{2} P\right)(P x \equiv P y) \supset x=y\right]\right) .
$$

It follows, accordingly, that the Russellian individuals that exist in any given possible world can be correlated one-to-one with the classes of $e$ properties those individuals have in that world (even though nonexisting individuals, having no $e$-properties in the world in question, are among themselves one and all indiscernible in their $e$-properties). Such classes of $e$-properties, needless to say, will represent the Meinongian objects that can be said to exist in that world; and, indeed, one suspects that it is only because of the above theorem and the fact that Meinongian objects can be "pictured" as classes of $e$-properties that the notion of existence has any bearing in the Meinongian framework at all.

As already indicated, Parsons's Meinongian theory $O$ is also a second order modal predicate logic, but unlike the Russellian theory it is based upon two sorts of ( $n$-place) predicate variables (and constants), one to range over nuclear properties and relations, and the other to range over extranuclear properties and relations. In this regard, one might well think it the purport of the theory, albeit one that can only be shown (through the theory's logical syntax) and not actually said in the theory itself, that no nuclear property or relation is also an extranuclear property or relation, respectively. This is not actually so, however, as Parsons himself observes (p. 88); and in fact it is a theorem of 0 that every nuclear property or relation is necessarily equivalent to an extranuclear property or relation. That is, taking the monadic case as a paradigm:

$$
\left(P^{N}\right)\left(\exists Q^{E}\right) \square(x)\left(P^{N} x \equiv Q^{E} x\right)
$$


is provable in $\mathbf{O}$. It follows, accordingly, at least with respect to modal contexts, that one can assume that every nuclear property or relation is also an extranuclear property or relation, respectively; and indeed there is nothing in the logic of propositional attitudes that would conflict with this assumption as well. Such an assumption, moreover, would dispell the temptation to distinguish two kinds of predication, viz., a Meinongian predication of nuclear properties and relations, and a Russellian predication of extranuclear properties and relations, since in that case the predication of a nuclear property or relation would also be the predication of an extranuclear property or relation.

Given the above assumption, needless to say, it would be pointless to distinguish between two sorts of ( $n$-place) predicate variables and constants, and quantification over extranuclear properties and relations would be none other than quantification over properties and relations in general, nuclear (or existence-entailing) and otherwise. This would also explain why the quantificational logic of extra-nuclear properties and relations is essentially that of standard second order modal predicate logic, and in particular-why a general comprehension principle for extranuclear properties and relations is provable in $\mathcal{O}$; that is, it explains why, e.g., in the monadic case

or

$$
\left(\exists P^{E}\right)[\lambda x \varphi]=P^{E}
$$

is a theorem schema of $\mathcal{O}$ (where $\varphi$ is a wff of $\mathcal{O}$ in which $P^{E}$ does not occur free). In addition, it would also explain why every Meinongian object is necessarily complete with respect to extranuclear properties even if not iwith respect to nuclear properties.

Instead of tw sorts of ( $n$-place) predicate variables and constants, we would, on the basis of the above assumption, need only introduce special quantifiers, say $\forall^{N}$ and $\exists^{N}$, for quantification over nuclear properties and relations; and the logic of these quantifiers, needless to say, would in that case amount to a "free" logic regarding nuclear properties and relations, which of course is as it should be if nuclear properties and relations are really $e$-properties and $e$-relations, and extranuclear properties and relations are properties and relationsingeneral, existence-entailingor otherwise. 
Our proposal, as already indicated, is fully borne out by the underlying predicate logic of extranuclear properties and relations, and in fact it explains why extranuclear properties and relations have the logic that they do. More than that, however, it also explains why nuclear properties and relations have the logic that they do. Thus, in particular, identifying nuclear properties and relations with $e$-properties and $e$-relations explains why the following thesis

$$
\left(P^{E}\right)\left(\exists Q^{N}\right) \square(x)\left(\mathrm{E} ! x \supset\left[Q^{N} x \equiv P^{E} x\right]\right)
$$

is valid or provable in 0 , since

$$
(\forall P)\left(\exists^{e} Q\right) \square(\forall x)[Q x \equiv P x \& E ! x]
$$

is already valid and provable in the Russellian theory in question. This latter thesis, moreover, has a natural explanation as a kind of Aussonderungsaxiom for $e$-properties-an explanation that is not available for nuclear properties when these are considered on their own within the Meinongian framework and independently of their Russellian interpretation.

The proof of the above thesis of 0 , incidentally, depends on Meinong's notion of the "watered down" nuclear version of an extranuclear property, a rather murky notion, as we have already indicated, about removing the "modal moment" from "full-strength factuality" (cf. [NO], 44). On Parsons's reconstruction this notion is applied to all extra-nuclear properties, as in the following "watering down" axiom of 0 :

$$
\left(P^{E}\right)(x)\left(\mathrm{E} ! x \supset\left[P^{E} x \equiv w\left(P^{E}\right) x\right]\right),
$$

which together with modal generalization, the Barcan wff (which is valid in both the Meinongian and Russellian theories), and the rule of grammar for 0 that $w\left(P^{E}\right)$ is a one-place nuclear predicate (and therefore can be existentially generalized upon as such) yields the thesis in question. It is clear, of course, that on the present proposal the "watering down" operation is completely superfluous, since in general the "watered down" nuclear version of an extranuclear property $\lambda x \varphi$, viz., $w(\lambda x \varphi)$, can be identified in the Russellian theory with the $e$-property $\lambda x(\varphi \& \mathrm{E} ! x)$.

This identification also explains why, moreover, the complement of a nuclear property or relation is not itself a nuclear property or relation, even though the "watered down" nuclear version of that complement is. 
That is, although in general the complement of an $e$-property or $e$ relation is not itself an $e$-property or $e$-relation, respectively, nevertheless, by the Aussonderungsaxiom, such a complement conjoined with existence does all the requisite work of the "watered down" nuclear version of that complement. This is but another example of how the natural and readily intuitive distinction between existence entailing properties and relations in general can explain what otherwise is a murky metaphysical notion applied to what otherwise would be an artificial distinction between nuclear and extranuclear properties and relations.

Finally, it should be noted that the one possible flaw in the present proposal is that it fails to account for Meinong's response to Russell regarding the existent present King of France. That is, on the present interpretation, being existent, even if construed as the "watered down" nuclear version of existence, is none other than existence (conjoined with) itself, and of course existence is itself an $e$-property par excellence. In this regard, there is no difference between existence as an extra-nuclear property and being existent as a nuclear property. (As already noted, the concept of existence in holistic conceptualism is not a predicative $e$-concept and differs in that regard from the predicative $e$-concepts ordinarily expressed in natural language. Meinong's example is not preserved even here, however, since being existent is also not a predicative $e$-concept. Moreover, since the assumption that every concept is necessarily equivalent with some predicative $e$-concept when restricted to existing individuals amounts to an unwarranted, and intuitively false, reducibility axiom, Parsons's "watering down" axiom would not be validated in holistic conceptualism.)

Notwithstanding the failure of our proposal to explain Meinong's example, nevertheless there is nothing in Parsons's reconstruction of Meinong's theory that shows that existence cannot be a nuclear property. Moreover, since Parsons has already rejected the application of the unrestricted satisfaction principle to all definite descriptions, it can hardly be of any real consequence to his reconstruction of Meinong's theory if we also reject his informal assumption that existence is an extra-nuclear property only. That is, if Meinong was wrong about so many other definite descriptions, why couldn't he be wrong about 'the existent present King of France'? In this regard, we would interpret (as most linguists probably would as well) 'the existent present King of France' as being but a stylistic 
variant of 'the present King of France that exists'; and, of course, this last definite description, as Parsons himself observes (cf. [NO], 170), is improper even when construed in terms of Parsons's reconstruction of Meinong's use of 'the'. Russell, in other words, was not "almost" on the mark, as Parsons claims, in his principal argument against Meinong. He was in fact exactly on the mark.

\section{Why Parsons's Plugging Up of Relations Is Unnecessary}

One of the nice things about the present proposal is that it eliminates the need to resort to Parsons's quite unintuitive and sometimes perplexing treatment of relations. Somehow the Russellian wisdom about the implausibility of reducing relations to relational properties is not appreciated by Parsons, and he formulates a grammar in which all $(n+1)$-place predicates must first be "plugged up" by singular terms in $n$ places before they can be predicated to generate an atomic wff (cf. [NO], 66). Parsons himself, it is clear, wants to give up this misdirected program of reducing the predication of relations between objects to the predication of relational properties, and his brief attempt to do so in a footnote (p. 67) makes it clear that he thinks of nuclear relations as $e$-relations. Our claim here is that if nuclear properties and relations really are $e$-properties and $e$ relations, then Parsons's program of reducing relations to relational properties is both unnecessary and unwarranted.

Where $R$ is a binary relation, for example, and $a$ and $b$ are Meinongian objects, Parsons distinguishes between the relational property $[a R]$, which is got by plugging up the first "place" of $R$ by $a$, and $[R b]$, which is got by plugging up the second "place" of $R$ by $b$ (cf. [NO], 59). An advocate of our Russellian theory would of course agree that there are relational properties here, but he would view them as derived and not as fundamental properties-which is the whole point, after all, of calling them relational properties. Relations, in other words, and the holding of relations between individuals, are a fundamental part of the Russellian framework, and they are not to be reduced or explained in terms of the possession of relational properties. In Parsons's reconstruction of Meinong's theory, on the other hand, it is the relational properties that are fundamental, and the holding of relations between objects is explained as but the conjunctive possession by these objects of the corresponding rela- 
tional properties. Indeed, according to Parsons it is even possible to have $a[R b]$ without also having $[a R] b$, which is completely counter to the Russellian view (cf. p. 59). No defense is made, however, of the claim that all relations are internal relations, which is the traditional reason for reducing relations to relational properties, and in fact that seems not to be Parsons's view at all.

This whole attempt to view the obtaining of relationships as but the possession of relational properties becomes unnecessary, as we have said, once nuclear properties and relations are identified with $e$-properties and $e$-relations. In particular, each of the following instances of Parsons's axiom for plugging up nuclear relations:

$$
\begin{aligned}
& \left(R^{N}\right)(x)(y)\left[\mathrm{E} ! x \supset\left(R^{N} x y \equiv\left[x R^{N}\right] y \& \mathrm{E} ! y\right)\right] \\
& \left(R^{N}\right)(x)(y)\left[\mathrm{E} ! y \supset\left(R^{N} x y \equiv x\left[R^{N} y\right] \& \mathrm{E} ! x\right)\right]
\end{aligned}
$$

is easily seen to correspond to a valid and provable thesis about $e$ relations in the Russellian theory in question. Of course, the fact that "for extra-nuclear relations, the order of plugging up is always irrelevant" ([NO], 159), i.e., the fact that

$$
\left(R^{E}\right)(x)(y)\left(x\left[R^{E} y\right] \equiv\left[x R^{E}\right] y\right)
$$

is valid in $\mathbb{O}$, is also easily explained by the identification of ( $n$-ary) extranuclear relations with ( $n$-ary) relations in general, existence entailing and otherwise, and the Russellian view of relational properties as derived properties.

Parsons, it might also be noted, has "omitted consideration of relations, such as worships, that are nuclear at the first place and extra-nuclear at the second place" ([NO], 65), but "this is only for simplicity" (ibid.). The real simplicity here, however, is all on the Russellian side, since among binary relations in general there are those like worship that entail existence with respect to their first "place" but not also with respect to their second, and these can easily be distinguished in the Russellian theory in question. Moreover, these may in fact be among the more important kinds of relations we ordinarily consider and represent in natural language, especially when temporal relations are allowed; and if this is so, then there is no reason, other than one's prejudice in favor of actualism, to give a special place to $e$-relations over those, e.g., that entail existence only with respect to 
their first "place." (The latter logic, incidentally, can be easily formulated and shown to be equipolent with the original.) Adding tense operators to our Russellian theory, moreover, and taking $e$-properties and $e$-relations, as well as properties and relations in general, to be temporal properties and relations is completely unproblematic (and in fact the original Russellian theory formulated in [ACT] and discussed in [EET] was constructed with just this sort of application in mind). Such an extension of our Russellian theory, needless to say, can be carried over to a temporal treatment of 0 on the basis of our present proposal; and in that regard, therefore, there really is no need to leave the temporal treatment of $\mathcal{O}$ as an open problem for future research, as Parsons in fact does (cf. [NO] 245).

\section{Properties and Relations as Individuals}

In a brief concluding section, Parsons considers the possibility of allowing properties, relations and propositions to be objects, but he rejects it on the basis of Russell's paradox of predication. This rejection is too hasty, however, since with a minor modification of the comprehension principle, viz., that it be homogeneously stratified (as defined below), Russell's paradox can be avoided.

The particular Russellian system we have in mind here is the one formulated in my "The Theory of Homogeneous Simple Types as a Second Order Logic," [THST], and referred to there as HST" (for Homogeneous Simple Types). This is a second order logic and not a type theory, it should be noted, even though it is equiconsistent with the simple theory of types. That is, other than the difference in type between individual and predicate variables (and constants), and the difference between $n$-place and $k$-place predicate variables (and constants) for distinct natural numbers $n$ and $k$, predicate expressions are not themselves typed as part of the grammar of the object language but are rather allowed to occur as singular terms in the same subject positions occupied by individual variables and constants in standard second order predicate logic. As singular terms, moreover, they are legitimate substituends of bound individual variables and can therefore be existentially generalized upon in their subject position occurrences by either first or second order quantifiers. In this regard the theory is said to be a second order logic only in the traditional or pretype-theoretical sense in which quantifiers are allowed to reach into both 
subject and predicate positions. The theory does of course contain standard second order logic as a proper part, but its higher order typetheoretical aspects occurs only in the metalanguage (the way it does for Quine's set theory NF). Thus, a wff $\varphi$ of HST* is said to be homogeneously stratified if there is an assignment $t$ of natural numbers to the variables and constants occurring in $\varphi$ such that for each atomic subwff $P\left(a_{0}, \ldots, a_{n-1}\right)$ of $\varphi,(1) t\left(a_{i}\right)=t\left(a_{j}\right)$, for all $i, j<n$, and $(2) t(P)=1+$ $t\left(a_{0}\right)$. (It is said to be stratified, though not necessarily homogeneously stratified, if clause (1) is dropped and clause (2) requires only that $t(P)=$ $1+\max \left[t\left(a_{0}\right), \ldots, t\left(a_{n-1}\right)\right]$; and it is said to be cumulatively stratified if $(1)$ is dropped and $(2)$ requires only that $\max \left[t\left(a_{0}\right), \ldots, t\left(a_{n-1}\right)\right]<t(P)$.)

As a modal free theory, the fundamental principle for positing properties, relations and propositions in $\mathrm{HST}^{*}$ is the following comprehension principle which stipulates that we take as an axiom of HST* each homogenously stratified instance of the schema:

$$
(\exists R)\left(\forall a_{1}\right) \ldots\left(\forall a_{n}\right)\left[R\left(a_{1}, \ldots, a_{n}\right) \equiv \varphi\right],
$$

where $\varphi$ is a wff of HST" in which $R$ does not occur (free) and each $a_{i}, 1$ $\leqslant i \leqslant n$, is either an individual or a predicate variable occurring free in $\varphi$. Note that this schema covers every instance of the comprehension principle of standard second order predicate logic. Moreover, it can be equivalently replaced by the different schema (which also must be homogeneously stratified):

$$
\begin{aligned}
& (\exists R)\left(\forall x_{1}\right) \ldots\left(\forall x_{n}\right)\left[R\left(x_{1}, \ldots, x_{n}\right) \equiv\right. \\
& \left.\left(\exists a_{1}\right) \ldots\left(\exists a_{n}\right)\left(x_{1}=a_{1} \& \ldots \& x_{n}=a_{n} \& \varphi\right)\right]
\end{aligned}
$$

where $\varphi, R, a_{1}, \ldots, a_{n}$ are as above, and $x_{1}, \ldots, x_{n}$ are all distinct individual variables; but in that case we will need to add

$$
\begin{aligned}
& \left(\forall R^{n}\right)\left(\forall S^{n}\right)\left(R=S \supset\left(\forall \mathrm{x}_{1}\right) \ldots\left(\forall x_{n}\right)\left[R\left(x_{1}, \ldots, x_{n}\right) \equiv\right.\right. \\
& \left.\left.S\left(x_{1}, \ldots, x_{n}\right)\right]\right)
\end{aligned}
$$

as a separate axiom. (This wff is provable on the basis of the original schema, but not on the basis of its replacement when identity is defined as indiscernibility.)

Adding modal operators (and the axioms of S5 modal propositional logic) to HST", the above schema would be replaced by all homogeneously stratified instances of: 


$$
(\exists R) \square\left(\forall a_{1}\right) \ldots\left(\forall a_{n}\right)\left[R\left(a_{1}, \ldots, a_{n}\right) \equiv \varphi\right]
$$

where $\varphi, R$, and $a_{1}, \ldots, a_{n}$ are as originally described. If the $\lambda$-abstraction operator is also added to HST", then we should make it a rule of the grammar that only homogeneously stratified $\lambda$-abstracts of the form:

$$
\left[\lambda x_{1} \ldots x_{n} \varphi\right]
$$

are to be well-formed $n$-place predicate expressions, where $x_{1}, \ldots, x_{n}$ are distinct individual variables and $\varphi, a_{1}, \ldots, a_{n}$ are as originally described. For it is only $\lambda$-abstracts of this form that can be applied as predicate expressions to arbitrary singular terms (including nominalized predicates). Note again that every $\lambda$-abstract of standard second order modal predicate logic (with $\lambda$-abstraction) is a well-formed predicate expression of the extended HST*. (See chapters 2 and 4 for further discussion of $\lambda \mathrm{HST}^{*}$, and for discussion of the related system HST $\mathrm{T}_{\lambda}^{*}$ as well, which is not covered in this chapter at all.)

Now it is noteworthy that neither of the following wffs

$$
\begin{aligned}
& (\exists P)(\forall Q)[P(Q) \equiv \sim Q(Q)] \\
& (\exists P)(\forall x)[P(x) \equiv(\exists Q)(x=Q \& \sim Q(x)]
\end{aligned}
$$

are homogeneously stratified, and therefore neither is an instance of the above comprehension principle (with or without modal operators). Both are disprovable, by Russell's argument for his paradox of predication, and in that regard the following wff

$$
(\exists R)(\forall P)(\forall x)[R(x, P) \equiv P(x)]
$$

is also disprovable in $\mathrm{HST}^{*}$, since we are able on its basis to prove Russell's paradox. (I am indebted to Edmund Gettier for pointing this out to me.) That is, according to HST ${ }^{*}$, predication does not, indeed cannot, stand for a relation.

Since the above wff regarding predication as a relation is stratified, but not homogeneously stratified, it follows that weakening the constraint on the comprehension principle so as to require only that its instances be stratified, but not necessarily homogeneously stratified, would result in an inconsistent theory; that is, in that case the above wff regarding predication would be both provable and disprovable. Of course, since wffs containing only monadic predicates are stratified if, and only if, they are ho- 
mogeneously stratified, monadic $\mathrm{HST}^{*}$ is the same as monadic $\mathrm{ST}^{*}$, where $\mathrm{ST}^{*}$ is $\mathrm{HST}^{*}$ with the constraint on the comprehension principle weakened as indicated. (It was this monadic ST" that was shown to be equiconsistent with the simple theory of types in [THST]; and HST" was shown to be equiconsistent with monadic $S T^{*}$.) Thus, even though the full $\mathrm{ST}^{*}$ system is inconsistent, monadic $\mathrm{ST}^{*}$ is not.

In regard to the question whether the constraint on the comprehension principle for monadic $\mathrm{HST}^{*}$ can be further weakened so as to require only that its instances be cumulatively stratified, it is easily seen that the resulting system, call it CST* (for cumulative simple types), is inconsistent. In particular, though the following wff

$$
(\exists P)(\forall x)[P(x) \equiv(\exists Q)(x=Q \& \sim Q(x))]
$$

is not stratified, it is nevertheless cumulatively stratified if identity is taken as indiscernibility (which it would be provably equivalent with in any case); and of course on the basis of Russell's argument for his paradox, this wff would be both provable and disprovable in CST*.

Now it is noteworthy that in standard type theories where the typing of predicates occurs as part of the grammar of the object language, we can prove the (relative) consistency not only of the theory of homogeneous simple types, but also of the full theory of heterogeneous simple types and even the theory of cumulative simple types. In contrast, as noted above, when the typing of predicates occurs only in the metalanguage and is not part of the grammar of the object language, then the second order theory corresponding to the theory of cumulative simple types is inconsistent; and although the monadic fragment of the second order theory corresponding to the theory of heterogeneous simple types is consistent, the full second order theory is not. In other words, it is only the full second order theory corresponding to the homogeneous theory of simple types that is consistent.

What is of philosophical interest, however, is that while the transition from the homogeneous to the heterogeneous to the cumulative theory of simple types (where the typing occurs as part of the grammar of the object language) marks an appropriate increasing generalization on the combined roles of predicates in predicate positions and predicates in subject positions, the generalization is still inadequate since the typing of predicates as part of the grammar of the object language is itself an artificial 
and limiting device (as Russell himself took it to be). Indeed, the appropriate and philosophically significant generalization occurs only when we remove these artificially imposed grammatical constraints and turn instead to the corresponding second order theories CST*, ST* and HST*. In this regard, since only $\mathrm{HST}^{*}$ is consistent in its relational part as well, it is only HST* that can be taken as an appropriate representative of Russell's original framework in which properties, relations and propositions are individuals on a par with concrete individuals.

Adding the special quantifiers $\forall^{e}$ and $\exists^{e}$ (and the axioms) for existenceentailing properties and relations to the modal extension of $\mathrm{HST}^{*}$ is quite unproblematic, it is clear, since these quantifiers are in fact definable if the existence predicate is taken as a primitive of the theory. Existence, of course, remains the prerogative only of concrete individuals, as Russell himself maintained; and in that regard concrete individuals can be distinguished as those individuals for which it is possible that they have an $e$ property, and abstract individuals are those that can never have an $e$-property or stand to other individuals in one or another $e$-relation.

Finally, though we shall not engage in the details of the proof for such here, it is clear that Parsons's model of the modal-free part of his Meinongian theory can be extended to his full theory and developed entirely within HST* as extended here. The model will not only validate all of Parsons's axioms as theorems of HST* so extended, but it will also provide a natural explanation of the somewhat artificial nuclear/extranuclear distinction of Parsons's theory and identify a natural source for extranuclear properties and relations as well.

\section{Will the Real Fictional Objects Please Stand Up}

Probably the best argument one can give for Meinongian objects, especially incomplete Meinongian objects, is their identification with the objects of dreams, fiction and myth-and perhaps even with the concrete objects of propositional attitudes, i.e., the concrete objects referred to within the scope of propositional attitude operators. Certainly in the case of fiction and myth the objects in question do not seem to be fully determinate with respect to the $e$-properties they are not said or understood to have in the fiction or myth in question, and perhaps the same might be said of the concrete objects referred to in dreams and propositional atti- 
tudes as well, i.e., that they are not fully determinate with respect to the $e$ properties they are not dreamed or otherwise thought to have in the context of the propositional attitudes in question. In this regard, it might well be claimed that unlike the semantics for tense and modal operators, the semantics for operators such as 'In the story', 'In the myth', 'In the dream', and perhaps even operators for belief and other propositional attitudes as well, should not be based on references to other possible worlds (or times) where only individuals that are fully determinate with respect to their $e$-properties can be said to exist.

A pre-1905 Russellian might of course reject this claim. There is, after all, at least one possible individual in the Russellian framework who in some possible world has all the $e$-properties that, e.g., Sherlock Holmes is said or thought to have in Conan Doyle's novels, just as there is a possible individual that in some possible world is a winged horse having all the $e$ properties Pegasus was said or thought to have in Greek mythology. These individuals do not exist, to be sure, and therefore do not actually have the $e$-properties in question, but they can nevertheless be referred to and perhaps even identified with the fictional Holmes and mythological Pegasus-or so it might be claimed.

The trouble with this proposal, of course, is that there are many possible individuals in many different possible worlds in the Russellian framework who have (in those worlds) all the $e$-properties that Sherlock Holmes is understood to have in Conan Doyle's novels, or all the $e$-properties that Pegasus was understood to have in Greek mythology; and it is not clear how exactly one such individual can be identified with the fictional Holmes or mythological Pegasus. None of these individuals, of course, will have only the $e$-properties that Holmes or Pegasus are said or thought to have, since being fully determinate existing individuals in their respective worlds, they will have many other $e$-properties as well. Moreover, even if the early (pre-1905) Russellian in question can resolve this problem, he will still have to account for stories and myths that are inconsistent and that seem to be about impossible objects, i.e., objects for which there are no suitable individuals with which they might be identified.

Without disputing these points, let us assume that this sort of approach to the semantics of operators for 'In the story', 'In the myth', 'In the dream', etc., will not do, and that in fact some other notion of an object must be resorted to, and that perhaps even Parsons's notion of a 
Meinongian object might be the most appropriate choice here. Having granted all that, nevertheless, it does not follow that the Russellian notion of an individual is now superfluous or that the concrete objects of fiction, myth, dream, etc., will have the $e$-properties they are said or dreamed to have in the fiction, myth, dream, etc. That is, the Russellian perspective is still fundamental here, and in particular having an $e$-property according to a story, myth, dream, etc., is in no way to be identified with having that $e$-property simpliciter, since the objects in question are not existing individuals. "Existing in fiction and existing," as Parsons himself observes, "are quite different things" ([NO], 50), and similarly having an $e$ property in a story is not the same as having that $e$-property simpliciter (cf. p. 53). Yet, having granted this much himself (except for using 'nuclear property' where we have used 'e-property'), Parsons nevertheless maintains that the fictional object Sherlock Holmes is a detective and that he "has exactly those nuclear properties which he is understood to have in the Conan Doyle novels" ([NO], 54), and, presumably, that the mythological object Pegasus is a winged horse and that it has exactly those $e$ properties it is said or understood to have in Greek mythology. That is, the $e$-properties of being a detective and being a horse apply no less so to the fictional Sherlock Holmes and mythological Pegasus than they do to existing individuals, a position that, at least in the Russellian framework, is absurd.

Now the point here is that if nuclear properties are really $e$-properties, then the concrete objects of fiction, myth, dream, etc., are not concrete Russellian individuals, existent or otherwise; that is, they are not individuals that in the full Russellian system of HST* can exist. Or, to put the point more positively, the concrete objects of fiction, myth, dream, etc., are abstract individuals. Which abstract individuals? Well, that depends on which of different Russellian alternatives one might take here. They can, e.g., be identified with certain classes of $e$-properties, which of course is just what Parsons "pictures" them as-given our identification of nuclear properties with $e$-properties. Classes need not themselves be fundamental or irreducible entities in HST, , however, and in fact the concrete objects in question might be better identified with certain properties of $e$-properties, viz., those that when considered as nominalized abstract. Russellian individuals play the same role in fiction, myth, etc., as Fregean individual concepts do in the context of a propositional attitude. (These 
properties of $e$-properties, needless to say, will correspond to functions on possible worlds to classes of $e$-properties.)

Parsons, apparently, would reject any Russellian interpretation of Meinongian objects here as not being the "intended" interpretation (cf. [NO], 92-7); but that may be because he sees a Russellian interpretation as "avoiding" reference to objects, which of course it cannot do (on our present reconstruction) if it is to account for the concrete objects of fiction, myth, dreams, etc. Posed as a question, Parsons's objection is that "if all reference to objects can be avoided, the existent as well as the nonexistent, what significance should this have for the special case of the nonexistent ones?" ([NO], 96). Well, the reference to objects is not avoided, as already noted, and reference to existing Meinongian objects, for reasons already given, is not at all the same as reference to existing Russellian individuals. (A property $P$ of $e$-properties is an "existing" Meinongian object if, and only if, there is an existing Russellian individual $x$ such that for all $e$-properties $Q, Q$ has $P$ if, and only if, $x$ has $Q$.) Existing Meinongian objects correlate, to be sure, one-to-one with existing Russellian individuals (but not in such a way that the correlation can identify the same individual in different possible worlds); but one cannot avoid referring to existing Russellian individuals by referring to existing Meinongian objects. After all, if nuclear properties really are $e$-properties, then there must be Russellian individuals to begin with that have $e$ properties in the sense in which they are $e$-properties, i.e., in the sense in which they are properties that entail existence. Understood in this way, it is clear how and why Meinongian objects are parasitic upon Russellian individuals, existent or otherwise, though they are nonetheless beneficial and have a job to do within the wider Russellian framework.

\section{References}

[EET] Cocchiarella, Nino B., "Existence Entailing Attributes, Modes of Copulation and Modes of Being in Second Order Logic," Nous 3 (1969), 33-48.

[ACT] Cocchiarella, Nino B., "A Completeness Theorem in Second Order Modal Logic," Theoria 2 (1969), 81-103.

[THST] Cocchiarella, Nino B., "The Theory of Homogeneous Simple Types as a Second Order Logic," Notre Dame Journal of Formal Logic 20 (1979), 505-24; and "Two $\lambda$-Extensions of the Theory Of Homogeneous Simple Types as a SecondOrder Logic," Notre Dame Journal of Formal Logic 26 (1983), 377-407. 
[PTQ] Montague, Richard M., "The Proper Treatment of Quantification in Ordinary English," in Approaches to Natural Language, J. Hintikka, J. Moravcsik and P. Suppes (eds.), D. Reidel, Doredrecht (1973) and reprinted in Formal Philosophy, R. H. Thomason (ed.), Yale University Press (1974).

[NO] Parsons, Terence, Nonexistent Objects, (New Haven and London: Yale University Press, 1980.)

[PMS] Parsons, Terence, "A Prolegomenon to Meinongian Semantics," Journal of Philosophy 71 (1974), 561-80.

[NEN] Parsons, Terence, "Nuclear and Extra-nuclear Properties, Meinong, and Leibniz," Nous 12 (1978), 137-50.

[POM] Russell, Bertrand, The Principles of Mathematics (1903), second edition with new introduction, Norton, N.Y., 1938.

[OD] Russell, Bertrand, "On Denoting," Mind, n.s. 14 (1905), 479-93. Reprinted in Essays in Analysis, D. Lackey (ed.), George Braziller Inc., N.Y., 1973. (Pagination is to this reprinting.)

[MTC] Russell, Bertrand, "Meinong's Theory of Complexes and Assumptions," Mind n.s. 13 (1904), 204-19, 336-54, and 509-24. Reprinted in Essays in Analysis. (Reference is made herein to the pagination of the latter.) 


\section{Frege's Double Correlation Thesis and Quine's Set Theories NF and ML}

There are two fundamentally different notions of a class, which, following tradition, we might call the mathematical and the logical notions, respectively. The logical notion is essentially the notion of a class as the extension of a concept, and, following Frege, we shall assume that a class in this sense "simply has its being in the concept, not in the objects which belong to it" (Frege, [PW], 183)-regardless of whether or not concepts themselves differ, as Frege assumed, "only so far as their extensions are different" (ibid., 118). The mathematical notion of a class, on the other hand, is essentially the notion of a class as composed of its members, i.e., of a class that has its being in the objects that belong to it. This notion of a class, we claim, is none other than the iterative concept of set-or at least that is what it comes to upon analysis. Note that although what accounts for the being of a class under the one notion is not the same as what accounts for the being of a class under the other, nevertheless the axiom of extensionality applies equally to both notions. This means that the axiom of extensionality does not of itself account for the being of a class. ${ }^{1}$

Of course the logical notion of a class, especially as developed in Frege's form of logicism, is usually thought to be bankrupt as a result of Russell's paradox. This assessment, however, is erroneous. In particular, in "Frege, Russell, and Logicism: A Logical Reconstruction," ([FRL]), I 
have explained how Frege's view of classes in the logical sense can be reconstructed without paradox by modifying in either of two ways what I there referred to as Frege's double correlation thesis. The two systems that result from these modifications, it turns out, have certain structural similarities with Quine's two set theories NF and ML, especially when the latter are themselves modified so as to include urelements other than the empty set. This is significant because both NF and ML are commonly said to "lack a motivation" (cf. Boolos's "The Iterative Concept of Set" ([ICS]), 219). But that is because as theories of sets in the sense of classes that are composed of their members, which is really the only sense to which Quine is willing to commit himself, both NF and ML are incompatible with the iterative concept of set. As theories of classes in the logical sense, however, and in particular of the classes that Frege took to be the correlates of concepts, both NF and ML can be given a very natural motivation, especially when modified to include urelements. In what follows we shall defend this motivation by examining the structural similarities in question.

\section{NF and the Iterative Concept of Set}

The original motivation for NF, according to Quine, was the reconstruction of the theory of simple types as a first order theory of classes, or what we shall also call a theory of sets in the sense of classes that are composed of their members. The theory of simple types, as a theory of classes, was already itself a reconstruction by Ramsey of Russell's earlier theory of ramified types as a theory of intensional concepts (or what Russell called propositional functions). The latter reconstruction was implicit in the interpretation Russell gave to his axiom of reducibility (cf. [PM], 58 and 166), but whereas for Ramsey classes were real and had their being in the objects that belonged to them, Russell chose not to assume that classes had any being at all but rather that all our talk of classes was to be explained in terms of concepts. Quine, of course, eschews all intensional entities and saw in the theory of simple types only a theory of sets in the sense of classes that are composed of their members. (For Quine, appar-

ently, the axiom of extensionality provides a full account of the being of a class.) The real ontological import of the theory of simple types, according to Quine, is a stratified comprehension principle for sets that it trivi- 
ally effects through its overly restrictive grammatical constraints. If we delete those grammatical constraints as being improperly retained through their historical connection with the intensional theory of ramified types, then what remains is none other than the stratified comprehension principle for sets given in NF.

Quine also assumed, but only as a "harmless" simplifying gesture, that the axiom of extensionality was to be applied to all objects whatsoever. It follows of course that there is then but one urelement, viz., the empty set. Objects that are not sets, according to Quine, can be simply identified with their singletons, though of course no commitment need actually be made in NF that there are any such objects at all. Stated as an applied first order theory (with identity), the system NF is really quite elegant in its simplicity.

But all is not well with NF as a theory of sets, i.e., as a theory of classes that are composed of their members. For the notion of a set as a class that is composed of its members, i.e., of a class that has its being in the objects that belong to it, is none other, we maintain, than the iterative concept of set. And NF, apparently, is incompatible with any theory that can be said to represent the iterative concept of set. Thus, the standard first order set theory that is taken as a paradigm of systems that represent the iterative concept of set is ZF (Zermelo-Fraenkel set theory ${ }^{2}$ ), and the incompatibility between $\mathrm{ZF}$ and NF is easy to demonstrate. In particular, in ZF there neither is nor can be a universal set, and therefore no set has an absolute complement. In addition, in ZF no set can be a member of itself. In NF, on the other hand, there is a universal set which, being universal, is therefore a member of itself; and of course every set has an absolute complement.

The guiding principle of ZF, incidentally, is what Russell once called the limitation of size doctrine, i.e. the doctrine that sets are not to get too big too fast (cf. Russell, "On Some Difficulties in the Theory of Transfinite Numbers and Order Types," ([OSD]), 152). According to this doctrine, we are "to admit only those instances of the axiom schema of comprehension which assert the existence of sets which are not too 'big' compared to sets which we already have" (Fraenkel and Bar-Hillel, Foundations of Set Theory, ([FST]), 32). Of course, it is just this doctrine that is realized by the iterative concept of set, since according to the latter sets are formed in stages by iterating the operation: $X$ $\rightarrow X \cup \mathscr{P}(X)$, where $\mathscr{P}(X)$ is the power set of $X$. It is by identifying the 
being of a set with its "formation" in this manner, incidentally, that we are able to say that the iterative concept of set is none other than the analysans of the mathematical notion of a set as a class that is composed of its members, i.e., of a class that has its being in the objects that belong to it.

Now the stages at which sets are formed are themselves well-ordered, and although the strongest form of the iterative concept of set assumes that for any given ordinal $\alpha$ there is an $\alpha$ th stage (cf. Wang, From Mathematics to Philosophy, ([FMP]), chapter 6), a weaker form assumes only that there is a stage corresponding to some limit ordinal (cf. [ICS], 221). But even this is not really essential to the concept (cf. Kriesel, "Two Notes on the Foundations of Set-Theory," ([TN]), 98), and in fact the theory of sets (sometimes also called general set theory) obtained from ZF by omitting the axiom of infinity might be said to represent the core form of the iterative concept. This is important, since if we exclude infinite stages and ignore the cumulative nature of the operation in question, then what we get by representing the stages by different types is essentially the theory of simple types as a theory of classes that are composed of their members. As a theory of classes, in other words, the theory of simple types represents a restricted form of the iterative concept of set, and in fact it represents a form that has its origin in Schröder's Algebra der Logik.

Schröder, as Alonzo Church has observed, anticipated the theory of types (as a theory of classes) when he took "the universal class 1 which appears in his algebra, not as an absolute universal class, but as composed of all the elements of a certain domain fixed in advance" (Church, "Schröder's Anticipation of the Simple Theory of Types." ([SA]), 150). Once such a quasi-universal class or "manifold" was given, a second may then be obtained (to which the algebra was to be applied in turn) "by taking the subsets of the first to be the individuals of the second" (ibid.); and by continuing in this way a "hierarchy of reine Mannigfaltigkeiten may be extended to infinity" (ibid.). The important restriction Schröder imposed on this hierarchy was that no subset of the domain of "individuals" considered at any one stage of the hierarchy was to be among the individuals of that stage, and that consequently the laws of logic, as the laws of this algebra, were to be restricted in any given application only to the "individuals" of the stage in question. Russell's first theory of types in Appendix B of his Principles of Mathematics was essentially this theory of 
classes, incidentally, though to what extent Russell was himself aware of this fact is not clear (cf. [FRL], section 8). That is, it was classes and not propositional functions to which Russell assigned types in that appendix; and the classes in question, moreover, were "classes as many," i.e., classes that essentially were nothing but their members. (It was not until after publication of the Principles that Russell adopted his famous " $n_{0}$ classes" theory.)

Now as a restricted form of the iterative concept of set, the theory of simple types as a theory of classes also realizes a restricted form of the limitation of size doctrine; and, indeed, it is for precisely this reason that an axiom of infinity to the effect that there are infinitely many urelements is needed in type theory. Also, just as the axiom of choice is neither provable nor disprovable in ZF (and therefore not really involved in the iterative concept of set), it is also neither provable nor disprovable in the theory of simple types as a theory of classes. Yet, in NF, which is supposed to be a first order reconstruction of the theory of simple types as a theory of classes, the axiom of choice is disprovable, and therefore, since the axiom is provable for finite sets, it follows that some set in NF is infinite (cf. Specker's, "The Axiom of Choice in Quine's New Foundations for Mathematical Logic," [AOC]). In NF, moreover, because it contains the Boolean calculus of classes, there is a complete symmetry between the existence or being of sets that are small and the existence or being of sets that are large; that is, the doctrine of the limitation of size is completely rejected in NF. It is no wonder then that as a theory of sets, i.e., as a theory of classes that are composed of their members, NF is said to "lack a motivation."

The alternative to this conclusion, of course, is that NF is really not a theory of sets in the sense of classes that are composed of their members. That is, if it has any philosophically coherent motivation at all, then it is as a theory of classes in the logical sense, i.e. in the sense in which classes have their being in the concepts whose extensions they are, and not as a theory of classes that have their being in the objects that belong to them. Such an alternative, it is clear, requires a background theory of concepts, and in particular a theory of predicable concepts whose extensions are classes in the logical sense. That is, such an alternative presupposes a superseding theory of predication in which predicates, despite their not being singular terms, can be said to have referential significance, and there- 
fore one in which quantification with respect to predicate positions can be said to be significant as well. This conclusion, needless to say, would not be welcome to one, such as Quine himself, who rejects the idea that predicate quantifiers can have any referential significance at all. For Frege, however, the notion that predicate quantifiers have a referential significance even though predicates are not themselves singular terms is not only acceptable but is in fact forced upon us by the very nature of language and thought (cf. [FRL], sections 1 and 2).

\section{The Theory of Simple Types and Frege's Double Correlation Thesis}

It is sometimes claimed, as Church observed (long before the view became fashionable), that Frege's Stufen or levels of concepts "constitute an anticipation of the simple theory of types" ([SA], 151). Church himself rejects this claim, however, and he is right in doing so if by the theory of simple types we mean either a theory of classes in the sense already described or a theory of concepts all of which can have classes as their extensions in the same sense in which first level concepts have classes as their extensions. Of course, in some sense Frege's theory of different levels of concepts does amount to a theory of types. But still, unlike the situation in Russell's theory of types where all propositional functions have extensions in the same sense in which the first order propositional functions that apply to concrete individuals do, in Frege's theory only first level concepts have or even can have extensions. To be sure, according to Frege, every concept of a given level falls within some concept of the next higher level; but still, no concept in Frege's hierarchy can be said to fall under a higher level concept the way that objects fall under first level concepts.

Nor is this merely a verbal distinction either! For example, second level concepts correspond not to predicates the way that first level concepts do, but to variable binding operators that apply to wffs (well-formed formulas) and result in wffs when so applied (such as the existential quantifier when affixed to an individual variable). (Equivalently, second level concepts correspond to wffs with free predicate variables that may be used in a third level comprehension principle to specify such a variable binding operator, such as the wff $\forall x[F(x) \rightarrow G(x)]$, which specifies the second level relation of subordination between one first level concept and an- 
other-cf. my "A Second Order Logic of Variable-Binding Operators," ([SOL]).) Unlike predicates, these operators can be commuted and iterated, as well as occur within the scope of one another. And of course the same observation applies to operators for third and higher level concepts, except that these operators can also be commuted with those for second level concepts, and therefore they can occur within the scope of the latter as well. Is this important? Indeed it is, for contrary to the view of the theory of types as a restricted form of the iterative concept of set, what it seemed to suggest to Frege is that there are no more third and higher level concepts than there are second level concepts; and, apparently, this in turn suggested to him that third and higher level concepts can be represented in a certain manner by second level concepts. That is why he saw no point in explicitly introducing notation for third and higher level concepts into his ideography (cf. [G\&B], 41). ${ }^{3}$

Frege also saw no point in introducing quantifiers regarding second level concepts; and the reason why is that he also assumed that there are no more second level concepts than there are first level concepts. That is, according to Frege, and this is what we mean by his double correlation thesis, all second level concepts can be correlated one-to-one with certain special first level concepts which in turn can be correlated one-to-one with special objects called concept-correlates. These concept-correlates are none other than Frege's value-ranges (Wertverläufe), which in the monadic case Frege also called classes (Begriffsumfangen). Thus quantifiers regarding second level concepts are unnecessary, according to Frege, because second level concepts "can be represented in a certain manner" by first level concepts whereby the concepts "that appear as arguments of the former are represented by their value-ranges" (Frege, The Basic Laws of Arithmetic, ([BLA]), Section 25). ${ }^{4}$ That is, in symbols (in the case of unary concepts):

$$
\forall Q \exists F \forall G[Q x G(x) \leftrightarrow F(\dot{\epsilon} G(\epsilon))] .
$$

In the relational case, incidentally, Frege's basic law V, viz.,

$$
\epsilon^{\prime} F(\epsilon)=\dot{\epsilon} G(\epsilon) \leftrightarrow \forall x[F(x) \leftrightarrow G(x)],
$$

is none other than a special instance of his double correlation thesis. For what is indicated on the right side of this law is none other than the second level relation of material equivalence or mutual subordination of two 
first level concepts; and on Frege's extensional view of concepts (as functions from objects to truth-values) such an equivalence amounts in effect to their "identity." That is, Frege's basic law V amounts to correlating the first level relation of identity with his second level relation of mutual subordination. Such a correlation is needed, Frege observes, since "to construe mutual subordination simply as equality is forbidden by the basic difference between first and second level relations. Concepts cannot stand in a first level relation. That wouldn't be false, it would be nonsense. Only in the case of objects can there be any question of equality (identity). And so the said transformation [from mutual subordination to identity] can only occur by concepts being correlated with the same object. It is all, so to speak, moved down a level" ([PW], 182, italics added).

Now just as first level concepts can be "moved down a level" by being correlated with their extensions, so too, by Frege's double correlation thesis, second level concepts can be moved down a level by being correlated with certain special first level concepts, which in turn of course are correlated with their own extensions. But then, third level concepts can be similarly "moved down a level" by being correlated with second level concepts, and therefore by the product of these correlations, third level concepts can in effect be correlated with first level concepts, which again are correlated with their own extensions. Similarly, fourth level concepts can be correlated with third level concepts and therefore with first level concepts as well. In general, in other words, all concepts of whatever level can in effect be correlated with first level concepts, and these in turn can be correlated with their extensions. Frege's entire hierarchy of concepts, accordingly, can be collapsed into the universe of first level concepts, which in turn can be correlated with the classes that are their extensions. In this regard, the theory of classes that is contained in Frege's theory of types of concepts is radically different from the theory of simple types as itself a theory of classes; i.e., it is radically different from the theory of simple types as a restricted form of the iterative concept of set.

Of course, Frege's theory, as originally described, is subject to Russell's paradox. But this result has nothing to do with the difference between Frege's hierarchy of concepts and the theory of simple types as a restricted form of the iterative concept of set. Indeed, by modifying in either of two ways Frege's double correlation thesis, we can reconstruct Frege's theory so that the resulting framework is provably consistent if the theory 
of simple types as a restricted form of the iterative concept of set is consistent. The two alternative systems resulting by these reconstructions, it turns out, are similar in structure to Quine's set theories NF and ML, and of course that suggests re-evaluating the latter in the light of our two modifications of Frege's double correlation thesis.

\section{Frege's Logicism as Second Order Predicate Logic with Nominalized Predicates}

Frege's original Begriffsschrift, as is well known, amounts to the first formulation ever given of standard second order predicate logic. It is clear, of course, that Frege viewed his system as providing a logical analysis of the predicates that occur in natural language. In the Grundgesetze, Frege supplemented this predicate logic with his smooth breathing abstraction operator, which when applied to wffs generated complex singular terms. These singular terms were of course taken as denoting value-ranges, which for reasons already indicated, Frege also called concept-correlates. What we have not yet noted, however, is that these concept-correlates are also none other than the objects that Frege took the nominalized predicates of natural language to denote (cf. [FRL], sections 4 and 5). In this regard, we maintain, Frege intended that his overall system was to provide a logical analysis not only of the predicates that occur in natural language but also of the predicate nominalizations that occur therein as well. For this, among other reasons, it was suggested in [FRL] that the confusion sometimes made of Frege's theory of value-ranges with a second order set theory might best be obviated by directly describing his theory of valueranges as a theory of concept-correlates in the sense of the objects that are denoted by nominalized predicates. In other words, Frege's form of logicism, we maintain, can best be described as a second order predicate logic with nominalized predicates. That, in any case, is the approach we shall adopt here.

In doing so, however, we shall for convenience allow ourselves certain liberties in the way we shall represent predicates and their nominalizations. In particular, we shall represent the unsaturated nature of predicates (at least when they occur as such) by the requirement that they be accompanied by a pair of parentheses (and commas as well in the case of a relational predicate). We shall then represent the nominalization of a predicate by the 
deletion of these parentheses (and the accompanying commas, if any). Thus, whereas $F(x)$ and $R(x, y)$ are wffs in which $F$ and $R$ occur as predicates, $G(F)$ and $G(R)$ are wffs in which $F$ and $R$ occur as singular terms. In $F(F)$ and $R(F, R)$, of course, $F$ and $R$ occur both as predicates and as singular terms (though no single occurrence can be both as a predicate and as $a$ singularterm). The predicate expressions that occur in the argument or subject positions of other predicates, or of themselves as well, needless to say, are intended as representing the nominalized predicates that occur in natural language. Note that adding such suffixes as '-ity', '-ness', or '-hood' to nominalized occurrences of predicates would be completely superfluous here since such occurrences are already formally identified as subject position occurrences; and of course the same observation applies to such related phrases as 'the concept $F$ ' or 'being an $F$ '. Such phrases and suffixes are important in English syntax, no doubt, since they serve to mark derived nominal expressions in the surface grammar of English; but it is sheer sophistry to insist that such surface grammatical features of English either must or should occur in our logical forms, as though a logical error were being committed otherwise.

In addition, we shall also use Church's $\lambda$-operator for the formation of complex predicates. (This is in keeping with most of the higher order predicate logics formulated in the recent literature.) Thus, where $\phi(x)$ is a wff, we also have $[\lambda x \phi(x)]($ ) as a complex predicate (note the accompanying pair of parentheses) and $[\lambda x \phi(x)]$ as a complex singular term. For convenience, however, we shall usually drop the accompanying parentheses (and commas) when referring to predicates (as unsaturated expressions), though always, whenever a predicate occurs in a wff as a predicate, it will have an accompanying pair of parentheses (and commas as well if it is a relational predicate).

Finally, in describing our logical grammar we shall for convenience identify the different types of meaningful expressions by associating them with different natural numbers. Thus we shall understand 0 to represent the type of all singular terms, 1 the type of all wffs or propositional forms, and $n+1$, for all $n>0$, as the type of all $n$-place predicate expressions. (We shall ignore the introduction of predicate and individual constants in the present paper.) We assume, of course, the availability of denumerably many individual variables, and, for each natural number $n$, denumerably many $n$-place predicate variables. (We identify proposi- 
tional variables with the 0 -place predicate variables.) We take ' $x$ ', ' $y$ ', ' $z$ ', and ' $w$ ', with or without numerical subscripts, to refer (in the metalan-

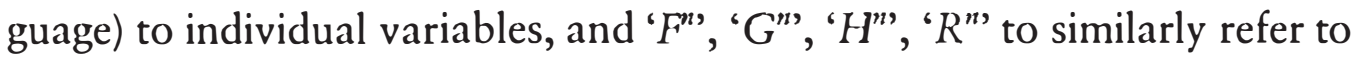
$n$-place predicate variables. (We shall usually drop the superscript when the context makes clear the degree of a predicate variable.) We shall also use ' $u$ ' to refer to variables in general, and ' $a$ ' and ' $b$ ' to refer to singular terms in general. We take $\rightarrow, \sim,=, \forall, \lambda$ as primitive logical constants and assume the others to be defined (as abbreviations of the metalanguage) in the usual way. For each natural number $n$, accordingly, we recursively define the meaningful expressions of type $n$, in symbols, $\mathrm{ME}_{n}$, as follows:

every individual variable (or constant) is in $\mathrm{ME}_{0}$, and every $n$-place predicate variable (or constant) is in both $\mathrm{ME}_{n+1}$ and $\mathrm{ME}_{0}$;

if $a, b \in \mathrm{ME}_{0}$, then $(a=b) \in \mathrm{ME}_{1}$;

if $\pi \in \mathrm{ME}_{n+1}$, and $a_{1}, \ldots, a_{n} \in \mathrm{ME}_{0}$, then $\pi\left(a_{1}, \ldots, a_{n}\right) \in$ $\mathrm{ME}_{1}$

if $\phi \in \mathrm{ME}_{1}$, and $x_{1}, \ldots, x_{n}$ are pairwise distinct individual variables, then $\left[\lambda x_{1} \ldots x_{n} \phi\right] \in \mathrm{ME}_{n+1}$;

if $\phi \in \mathrm{ME}_{1}$, then $\sim \phi \in \mathrm{ME}_{1}$;

if $\phi, \psi \in \mathrm{ME}_{1}$, then $(\phi \rightarrow \psi) \in \mathrm{ME}_{1}$;

if $\phi \in \mathrm{ME}_{1}$, and $a$ is an individual or a predicate variable, then $\forall a \phi \in \mathrm{ME}_{1}$;

if $\phi \in \mathrm{ME}_{1}$, then $[\lambda \phi] \in \mathrm{ME}_{0}$; and

if $n>1$, then $\mathrm{ME}_{n} \subseteq \mathrm{ME}_{0}$.

Singular terms, which we shall also refer to simply as terms, are understood to be all the members of $\mathrm{ME}_{0}$; and for $n>0$, we understand the members of $\mathrm{ME}_{n+1}$ to be $n$-place predicate expressions. Wffs or propositional forms are of course all the members of $\mathrm{ME}_{1}$. Note that whereas by clause (9) every predicate expression is a term, not every wff is a term. We differ in this regard from what Frege would allow; but the difference is unimportant since by clause (4), where $n=0,[\lambda \phi]$ is a wff if $\phi$ is a wff, and by clause $(8)[\lambda \phi]$ is a term. In other words, besides 0 -place predicate vari- 
ables (and constants), wffs are terms only when prefixed by the $\lambda$ operator. Thus, in general, we may read ' $[\lambda \phi]$ ' as 'that $\phi$ ' when it occurs in a wff as a term, i.e., when it occurs in one of the argument or subject positions of a predicate expression.

If we take this logical grammar as a counterpart to Frege's own original ideography, then, ignoring Russell's paradox for the present, the axioms of standard second order predicate logic when applied to this grammar can be taken as the counterparts to Frege's basic laws of logic. The following axiom set will serve us for this purpose. (Of course we include an axiom for $\lambda$-conversion, and an axiom that identifies what a nominalized predicate variable - or constant-denotes with what its corresponding nominalized $\lambda$-abstract denotes. Also, we add a "-label to all theses that are understood to include wffs having nominalized predicates as singular terms among their instances. Bondage and freedom are understood defined in the usual way.)

$\left(\mathrm{A} 0^{*}\right) \quad$ all tautologous wffs,

(A1") $\quad \forall u(\phi \rightarrow \psi) \rightarrow(\forall u \phi \rightarrow \forall u \psi \psi)$, where $u$ is an individual or predicate variable,

(A2*) $\quad \phi \rightarrow \forall u \phi$, where $u$ is an individual or predicate variable not occurring free in $\phi$,

$\left(\mathrm{A} 3^{*}\right) \quad \exists x(a=x)$, where $a$ is a singular term in which $x$ does not occur free,

$\left(\mathrm{LL}^{*}\right) \quad(a=b) \rightarrow(\phi \leftrightarrow \psi)$, where $a, b$ are singular terms and $\psi$ comes from $\phi$ by replacing one or more free occurrences of $b$ by free occurrences of $a$,

$\left(\mathrm{CP}^{n}\right) \quad \exists F^{n} \forall x_{1} \ldots \forall x_{n}\left[F\left(x_{1}, \ldots, x_{n}\right) \leftrightarrow \phi\right]$, where $F^{n}$ does not occur free in $\phi$ and $x_{1}, \ldots, x_{n}$ are distinct individual variables,

$\left(\lambda\right.$-Conv") $\quad\left[\lambda x_{1} \ldots x_{n} \phi\right]\left(a_{1}, \ldots, a_{n}\right) \leftrightarrow \phi\left(a_{1} / x_{1}, \ldots, a_{n}\left(x_{n}\right)\right.$, where $a_{1}, \ldots, a_{n}$ are singular terms, and each $a_{i}$ is free for $x_{i}$ in $\phi$,

$\left(\mathrm{Id}_{\lambda}^{*}\right) \quad\left[\lambda x_{1} \ldots x_{n} R\left(x_{1}, \ldots, x_{n}\right)\right]=R$, where $R$ is an $n$-place predicate variable (or constant). 
As inference rules, we shall assume modus ponens ( $\mathrm{MP}$ ) and universal generalization (UG) as applied to an individual or a predicate variable. Note that by Leibniz's law, i.e. $\left(\mathrm{LL}^{*}\right)$, and $(\mathrm{A} 0 *)-(\mathrm{A} 3 *)$, the principle of universal instantiation of a singular term for an individual variable is provable:

$$
\forall x \phi \rightarrow \phi(a / x)
$$

This is essentially Frege's basic law (IIa). Frege's basic law (IIb) is similarly derivable from the second order counterpart of (LL*) and by using $\left(\mathrm{CP}^{*}\right)$ instead of $(\mathrm{A} 3 *)$. Note also, incidentally, that $\left(\mathrm{LL}^{*}\right)$ and $(\lambda$-Conv*) together yield the following generalized form of Frege's basic law $(\mathrm{Vb})$ :

$$
\left[\lambda \mathrm{x}_{1} \ldots x_{n} \phi\right]=\left[\lambda x_{1} \ldots x_{n} \psi\right] \rightarrow \forall x_{1} \ldots \forall x_{n}(\phi \leftrightarrow \psi) .
$$

Of course, this is only one direction of Frege's basic law V. The other direction, i.e. Frege's basic law (Va), is the following principle of extensionality, which we shall consider separately from the other axioms:

$$
\forall x_{1} \ldots \forall x_{n}(\phi \leftrightarrow \psi) \rightarrow\left[\lambda x_{1} \ldots x_{n} \phi\right]=\left[\lambda x_{1} \ldots x_{n} \psi\right] .
$$

Finally, although we have not included variables for variable binding operators representing second level concepts, note that were such variables available, we could represent Frege's double correlation thesis as follows:

$$
\forall Q \exists F \forall G[Q x G(x) \leftrightarrow F(G)] .
$$

Now since $Q x G(x)$ can also be taken as an abbreviation of a wff that contains $G$ as a free 1-place predicate variable, i.e., a wff which could be used to specify a second level concept $Q$ in a third level comprehension principle, then, as Frege himself noted, we can ignore explicitly introducing quantifiers for second level concepts and capture the correlation in question as an instance of $\left(\mathrm{CP}^{\prime *}\right)$ instead. For by $\left(\mathrm{CP}^{*-}\right)$,

$$
\exists F \forall x(F(x) \leftrightarrow \exists G[x=G \& Q x G(x)]),
$$

and therefore by $\left(\mathrm{UI}_{\mathrm{i}}^{*}\right)$ and other obvious transformations,

$$
\exists F \forall G[F(G) \leftrightarrow Q x G(x)] .
$$

In other words, every instance of Frege's double correlation thesis can be captured as an instance of $\left(\mathrm{CP}^{\text {\% }}\right)$. 
It is clear, accordingly, that together with (Ext*), the above system of axioms and rules amounts to a counterpart of Frege's own original system. Of course, like Frege's original system, the above system, with or without (Ext*), is subject to Russell's paradox and therefore is inconsistent. That is, by the following instance of $\left(\mathrm{CP}^{*}\right)$,

$$
\exists F \forall x(F(x) \leftrightarrow \exists G[x=G \& \sim G(x)]),
$$

a contradiction is provable in the system. Nevertheless, the framework is repairable, and by a simple modification of Frege's double correlation thesis as well; i.e., by a simple modification of ( $\left.\mathrm{CP}^{*}\right)$ as the representation of Frege's thesis in the present context. Before turning to this modification, however, let us note that $\left(\mathrm{CP}^{*}\right)$ is a consequence of a still simpler form of comprehension principle; namely,

$\left(\mathrm{CP}_{\lambda}^{*}\right) \quad \exists F^{n}\left(\left[\lambda x_{1} \ldots x_{n} \phi\right]=F\right)$,

where $F^{n}$ does not occur free in $\phi$. Since a restricted form of $\left(\mathrm{CP}_{\lambda}\right)$ will yield only a restricted form of $\left(\mathrm{CP}^{*}\right)$, we shall apply our modification to $\left(\mathrm{CP}_{\lambda}^{*}\right)$ instead. Note, however, that since $\left(\mathrm{CP}_{\lambda}^{*}\right)$ posits the existence of a concept corresponding to any given $\lambda$-abstract, then the restriction in question really amounts to a restriction as to which $\lambda$-abstracts are to be regarded as well-formed in the first place.

\section{The Theory of Homogeneous Simple Types as a Second Order Predicate Logic}

Now the point of our first modification of Frege's double correlation thesis is that if all higher level concepts can really be correlated with first level concepts, which in turn are correlated with their concept-correlates, then the conditions for specifying the first level concepts in question must be stratified in a way corresponding to the stratification of the higher level concepts to which those first level concepts and their conceptcorrelates correspond. The comprehension principle $\left(\mathrm{CP}_{\hat{\lambda}}^{*}\right)$, in other words, must be restricted in a way that corresponds to the stratification of the unsaturated concepts of Frege's hierarchy.

For reasons indicated below, however, we shall actually need a more stringent form of stratification than Frege allows. In particular, we shall be required to assume, on pain otherwise of generating Russell's paradox 
after all, that higher level relations are only homogeneously stratified. This in fact is the only modification of Frege's original form of logicism that we shall assume in our first reconstruction of Frege's logic.

Returning to the logical grammar of section 3, accordingly, let us say that a wff or $\lambda$-abstract $\phi$ of that grammar is homogeneously stratified iff there is an assignment $t$ of natural numbers to the set of terms occurring in $\phi$ (including $\phi$ itself if $\phi$ is a $\lambda$-abstract) such that (1) for all terms $a$ and $b$, if $(a=b)$ occurs in $\phi$, then $t(a)=t(b)$; (2) for all $n \geqslant 1$, all $n$-place predicate expressions $\pi$ and all terms $a_{1}, \ldots, a_{n}$, if $\pi\left(a_{1}, \ldots, a_{n}\right)$ is a wff occurring in $\phi$, then (i) $t\left(a_{j}\right)=t\left(a_{k}\right)$, for $1 \leqslant j, k \leqslant n$, and (ii) $t(\pi)=t\left(a_{1}\right)+1$; and (3) for all natural numbers $m$, all individual variables $x_{1}, \ldots, x_{m}$, and all wffs $\psi$, if $\left[\lambda x_{1} \ldots x_{m} \psi\right]$ occurs in $\phi$, then (iii) $t\left(x_{\mathrm{i}}\right)=t\left(x_{k}\right)$, for $1 \leqslant j, k \leqslant m$, and (iv) $t\left(\left[\lambda x_{1} \ldots x_{m} \psi\right]\right)=t\left(x_{1}\right)+1$. If clauses (i) and (iii) are dropped and clauses (ii) and (iv) are replaced by the weaker requirement that $t(\pi)=1$ $+\max \left[t\left(a_{1}\right), \ldots, t\left(a_{n}\right)\right]$ and $t\left(\left[\lambda x_{1} \ldots x_{m} \psi\right]\right)=1+\max \left[t\left(x_{1}\right), \ldots, t\left(x_{m}\right)\right]$, then we shall say that $\phi$ is heterogeneously stratified. Of course, by definition, every homogeneously stratified expression is heterogeneously stratified; and although not all heterogeneously stratified expressions are homogeneously stratified, nevertheless, a monadic expression, i.e. one in which every predicate is a 1-place predicate, is homogeneously stratified if and only if it is heterogeneously stratified. For this reason, homogeneously stratified monadic wffs will be referred to hereafter simply as stratified wffs.

Now it is clear that Frege's hierarchy of concepts is heterogeneously stratified and not just homogeneously stratified; i.e., that some of Frege's higher level relations are unequal leveled relations. Thus, e.g., the second level relation of an object to a concept under which that object falls is said by Frege to be an unequal second level relation because it has as arguments both a saturated object and an unsaturated first level concept (cf. [BLA], section 22). The relevant modification of his double correlation thesis, accordingly, would seem to be that we are to admit as well-formed only those $\lambda$-abstracts that are heterogeneously stratified. This would certainly exclude the version of Russell's paradox described above, since the complex predicate in question, viz. $[\lambda x \exists G(x=G \& \sim G(x))]$, is not heterogeneously stratified.

Unfortunately, this proposal does not also exclude other forms of 
Russell's paradox generated in terms of unequal leveled concepts. For example, since Frege's unequal leveled relation of subsumption under a concept is specified by a heterogeneously stratified $\lambda$-abstract, viz., $[\lambda x y \exists G(x$ $=G \& G(y))$ ], then being impredicable with respect to this relation is also specifiable in terms of such a $\lambda$-abstract, viz. $[\lambda z \sim[\lambda x y \exists G(x=G \&$ $G(y))](z, z)]$. But then Russell's paradox of the concept that is subsumed under itself if and only if it is not subsumed under itself is derivable after all. It follows, accordingly, that if Frege's double correlation thesis is to apply to all higher level unsaturated relations, then we must assume that none of those relations are inhomogeneously stratified, i.e., that there are no unequal higher level relations. In particular, we must not assume that there can be such an unequal second level relation as the subsumption of an object under a concept; for it is fundamental to Russell's paradox that predication cannot stand for a relation between an object and a concept-correlate.

On the modification in question, accordingly, a $\lambda$-abstract is considered as well-formed if and only if it is homogeneously stratified; and, in general, let us say that a meaningful expression of type $n$ is a meaningful expression of $\lambda \mathrm{HST}^{*}$ (where $\lambda \mathrm{HST}^{*}$ is the "version of the theory of homogeneous simple types) if and only if every $\lambda$-abstract occurring in that expression is homogeneously stratified. (Note that this does not require that wffs themselves be homogeneously stratified; in particular, $F(F)$ and $\sim F(F)$ are both wffs of $\lambda \mathrm{HST}^{*}$, and if $[\lambda x \phi]$ is homogeneously stratified, then both $[\lambda x \phi]([\lambda x \phi])$ and its negation are wffs of $\lambda \mathrm{HST}^{*}$ as well.) By an axiom of $\lambda \mathrm{HST}^{*}$, moreover, let us understand any wff of $\lambda \mathrm{HST}^{*}$ that is an instance of $\left(\mathrm{A}^{*}\right)-\left(\mathrm{A} 3^{*}\right),\left(\mathrm{LL}^{*}\right),\left(\mathrm{CP}_{\lambda}^{*}\right),\left(\lambda\right.$-Conv $\left.{ }^{*}\right)$ and $\left(\mathrm{Id}_{\lambda}^{*}\right)$, i.e. of the axioms already described in section 3. For convenience, we shall refer to the restriction of $\left(\mathrm{CP}_{\lambda}\right)$ that is effected hereby as the homogeneously stratified comprehension principle, or more simply as ( $\left.\mathrm{HSCP}{ }_{\lambda}^{*}\right)$. Our proposal or first alternative reconstruction of Frege's form of logicism, accordingly, is that it be restricted to the meaningful expressions of $\lambda \mathrm{HST}^{*}$ and that it be represented by the axiom system $\lambda \mathrm{HST}^{*}+\left(\mathrm{Ext}^{*}\right)$. (We assume, as before, that (MP) and (UG) are the only rules of inference. ${ }^{5}$ )

We need not follow Frege in construing concepts extensionally, incidentally. For example, we could also take $\square$ as a primitive logical constant and add to the axioms of $\lambda \mathrm{HST}^{*}$ the axiom schemas of the S5 modal propositional logic (and the rule of modal generalization as well). In that 
case, instead of (Ext*) we might assume the corresponding principle of intensionality:

$\left(\square \mathrm{Ext}{ }^{*}\right) \quad \square \forall x_{1} \ldots \forall x_{n}(\phi \leftrightarrow \psi) \rightarrow\left[\lambda x_{1} \ldots x_{n} \phi\right]=\left[\lambda x_{1} \ldots x_{n} \psi\right]$.

If we refer to this extension of $\lambda \mathrm{HST}^{*}$ as $\square \lambda \mathrm{HST}^{*}$, then Russell's idea of reducing all talk of classes to talk of concepts can be captured in terms of the following notion of rigidity: ${ }^{6}$

$$
\text { Rigid }=\mathrm{df}[\lambda x \exists F(x=F \& \forall y[\square F(y) \mathbf{v} \square \sim F(y)])] .
$$

For a class, on this analysis, is simply the correlate of a rigid intensional concept:

$$
C l s=\mathrm{df}[\lambda x \exists F(x=F \& \operatorname{Rigid}(F))] .
$$

The "law of logic" that is needed in that case is the following principle of rigidity:

$$
\forall F \exists G(C l s(G) \& \forall x[F(x) \leftrightarrow G(x)]) .
$$

It is clear, of course, that by translating $\square$ as double negation, $\square \lambda$ HST $^{*}+$ $\left(\square \mathrm{Ext}^{*}\right)+(\mathrm{PR})$ reduces to just $\lambda \mathrm{HST}^{*}+\left(\mathrm{Ext}^{*}\right)$, and therefore since the latter is translatable into the former it follows that the two systems are equiconsistent. $^{\text {? }}$

It should be noted in this context, incidentally, that in $\square \lambda H S T^{*}$ not all concepts are rigid, and therefore not all concept-correlates are "classes." They might, of course, be none other than the intensional concepts themselves-that is, if, along with Russell, we assume that the latter do not have an unsaturated nature after all. In that case, $\square \lambda \mathrm{HST}^{*}+\left(\square \mathrm{Ext} \mathrm{t}^{*}\right)+$ (PR) might well be taken as representing a form of logicism that Russell himself seemed to be defending in his 1902-1904 correspondence with Frege (cf. [FRL] for a defense of this claim). There are other options here as well, however, but we shall not discuss them in the present paper (cf. [FRL], section 15). In addition, we might even forego assuming either $\left(\mathrm{Ext}^{*}\right)$ or $\left(\square \mathrm{Ext}{ }^{*}\right)$, though in that case the idea of classes as conceptcorrelates (whether of all concepts or of only rigid concepts) must then be discarded. ${ }^{8}$ 
5. Quine's Thesis and the Similarity of NF with $\lambda H S T^{*}+$ $\left(E x t^{*}\right)+\left(Q^{*}\right)$

Although motivated in terms of different notions of a class, the similarity between Quine's "set" theory NF and $\lambda \mathrm{HST}^{*}+\left(\mathrm{Ext}^{*}\right)$ as the result of our first modification of Frege's double correlation thesis should by now be obvious. We shall examine the details of this similarity by restricting ourselves to monadic $\lambda \mathrm{HST}^{*}+\left(\mathrm{Ext}^{*}\right)$. (The restriction is inessential, since monadic $\lambda \mathrm{HST}^{*}+\left(\right.$ Ext $\left.^{* *}\right)$ is equiconsistent with the full system-cf. my Logical Investigations of Predication Theory and the Problem of Universals, $([L I])$, chapter 4 , section 8.$)$

First, let us note that since urelements other than the empty class are represented in NF by their singletons or unit classes, then we shall have to make a comparable assumption for $\lambda \mathrm{HST}^{*}+\left(\right.$ Ext $\left.^{*}\right)$. Although the assumption we give is actually simpler than Quine's, we shall nevertheless refer to it as Quine's thesis. It is the assumption that every object is a concept-correlate:

$\left(\mathrm{Q}^{*}\right) \quad \forall x \exists F(x=F)$.

Our initial result, accordingly, is that by defining membership as follows,

$$
x \in y=\mathrm{df} \exists F[y=F \& F(x)],
$$

every theorem of NF is a theorem of $\lambda \mathrm{HST}^{*}+\left(\mathrm{Ext}^{*}\right)+\left(\mathrm{Q}^{*}\right)$; i.e, NF is contained in $\lambda \mathrm{HST}^{*}+\left(\mathrm{Ext}^{*}\right)+\left(\mathrm{Q}^{*}\right)$ and therefore NF- "sets" can be construed as Fregean concept-correlates. (We shall hereafter assume this analysis of membership when referring to classes as concept-correlates, i.e., as the objects denoted by nominalized predicates. Since stratification in NF coincides under this analysis with stratification in $\lambda \mathrm{HST}^{*}$, incidentally, we shall not bother redefining it for first order wffs with $\in$ as a primitive 2-place predicate constant.)

By way of proof of this result, note that by definition,

$$
\overleftarrow{\mathrm{AHST}^{*}} x \in F \leftrightarrow F(x),
$$

and therefore since by applying (UG) to (A3*),

$$
\widehat{\mathrm{AHST}^{*}} \forall F \exists y(F=\mathrm{y}),
$$

then by ( $\left.\mathrm{HSCP}_{\lambda}^{*}\right)$, 


$$
\overleftarrow{\lambda F I^{*} *} \exists y \forall x(x \in y \leftrightarrow \phi),
$$

where $\phi$ is a stratified monadic wff in which $y$ does not occur free. It follows, accordingly, that every instance of the stratified comprehension principle for NF-"sets" (when membership is analyzed as above) is a theorem of $\lambda \mathrm{HST}^{*}$. In regard to the extensionality axiom,

$$
\forall x \forall y(\forall z[z \in x \leftrightarrow z \in y] \rightarrow x=y),
$$

we note that in fact

$$
\left(\mathrm{Ext}^{*}\right) \overline{\lambda I I S T^{* *}}\left(\mathrm{Q}^{*}\right) \leftrightarrow(\mathrm{Ext}),
$$

and therefore that Quine's thesis $\left(\mathrm{Q}^{*}\right)$ is provably equivalent to the axiom of extensionality for NF- "sets" (which is another reason for calling it Quine's thesis). Since the proof proceeds by obvious transformations, we won't go into the details here (especially since they can already be found in my "The Theory of Homogeneous Simple Types as a Second Order Logic," ([THST]), 511).

Besides NF being contained in $\lambda \mathrm{HST}^{*}+\left(\mathrm{Ext}^{*}\right)+\left(\mathrm{Q}^{*}\right)$, it is clear that by (mis)construing predicates as singular terms and interpreting predication as membership, every theorem of $\lambda \mathrm{HST}^{*}+\left(\mathrm{Ext}^{*}\right)+\left(\mathrm{Q}^{*}\right)$ will be translated into a theorem of NF. In other words, $\lambda H S T^{*}+\left(E x t^{*}\right)+\left(Q^{*}\right)$ and each of its subsystems is consistent relative to NF.

In defining the translation function in question, incidentally, we shall make use of the abbreviatory abstraction notation Quine introduces in his discussion of NF and ML. The abbreviations in question, it should be noted, are actually more appropriate for ML than NF, since they essentially involve the notion of being an element. Nevertheless, since all NF"sets" are elements, the addition of this notion is inessential when applied to NF, and defining the abbreviations this way enables us to forego revising them in our later discussion of ML. The abbreviations, accordingly, are as follows:

$$
\begin{array}{ll}
x \in \hat{x} \phi=\mathrm{df} & \exists z(x \in z) \& \phi, \\
z=\hat{x} \phi=\mathrm{df} & \forall x(x \in z \leftrightarrow x \in \hat{x} \phi), \\
\hat{x} \phi \in y=\mathrm{df} & \exists z(z=\hat{x} \phi \& z \in y) .
\end{array}
$$


Now let -(bar) be a one-to-one mapping of the individual and 1-place predicate variables onto the individual variables. We define a translation function $s$ on the basis of this mapping by means of a recursion on the meaningful expressions of monadic $\lambda \mathrm{HST}^{*}$ as follows. ${ }^{9}$

$$
\begin{aligned}
& \text { if } a, b \in \mathrm{ME}_{0}, \text { then } s(a=b)=(s(a)=s(b)), \\
& \text { if } a \in \mathrm{ME}_{0} \text { and } \pi \in \mathrm{ME}_{2} \text {, then } s(\pi(a))=(s(a) \in s(\pi)), \\
& s([\lambda x \phi])=\hat{y} s(\phi), \text { where } y=\bar{x}, \\
& s(\sim \phi)=\sim s(\phi), \\
& s(\phi \rightarrow \psi)=(s(\phi) \rightarrow s(\downarrow)), \\
& s(\forall u \phi)=\forall \bar{u} s(\phi), \\
& \text { if } \phi \in \mathrm{ME}_{1} \text {, then } s([\lambda \phi])=\hat{x}(s(\phi) \& x=x) .
\end{aligned}
$$

We observe that if $\phi$ is a monadic wff of $\lambda \mathrm{HST}^{*}$ that is an instance of either $\left(\mathrm{A} 0^{*}\right),\left(\mathrm{A} 1^{*}\right),\left(\mathrm{A} 2^{*}\right),\left(\mathrm{A} 3^{*}\right),\left(\mathrm{LL}^{*}\right)$, or $\left(\mathrm{Q}^{*}\right)$, then by definition $s(\phi)$ is provable in standard first order predicate logic with identity, and therefore $s(\phi)$ is a theorem of NF. On the other hand, if $\phi$ is an instance of (HSCP $\left.{ }^{*}\right)$, then $s(\phi)$ is of the form $\exists z(\hat{x} \psi=z)$, and therefore by (D2), (D1), and the stratified comprehension principle of NF, together with the fact that every NF- "set" is an element, it follows that $s(\phi)$ is a theorem of NF. Similarly, that the $s$-transform of every instance of $(\lambda$-Conv" $)$ is a theorem of NF also follows from (D1) and the fact that every NF-"set" is an element. If $\phi$ is an instance of ( $\operatorname{Id} *)$, then $s(\phi)$ is of the form $\hat{x}(x \in a)=$ $a$, and therefore by (D2) and (D1), it follows that $s(\phi)$ is a theorem of NF. A similar observation applies to the $s$-transforms of instances of (Ext*), moreover, and therefore since (MP) and (UG) preserve theoremhood in $\mathrm{NF}$, it follows that the $s$-transform of each theorem of monadic $\lambda \mathrm{HST}^{*}+$ $\left(\mathrm{Ext}^{*}\right)+\left(\mathrm{Q}^{*}\right)$ is a theorem of NF. Putting our two results together, we can now state the following metatheorem.

METATHEOREM $1 . \mathrm{NF}$ is equiconsistent with monadic $\lambda \mathrm{HST}^{*}+\left(\mathrm{Ext}^{*}\right)+$ $\left(\mathrm{Q}^{*}\right)$; and therefore since monadic $\lambda \mathrm{HST}^{*}+\left(\mathrm{Ext}^{*}\right)+\left(\mathrm{Q}^{*}\right)$ is equiconsistent with the full system, NF is equiconsistent with $\lambda \mathrm{HST}^{*}+\left(\mathrm{Ext}^{*}\right)+$ $\left(Q^{*}\right)$. 
Before concluding this section, it is worth noting that Ernst Specker has shown that NF is equiconsistent with the theory of simple types as a theory of classes if we add to the latter the assumption that all of the classes of any one type can be correlated one-to-one with the classes of the next succeeding type and that all of the classes of urelements can be correlated one-toone with those urelements-an assumption that Specker calls "complete typical ambiguity" (cf. Specker "Typical Ambiguity," ([TA]), 118). Such as assumption, needless to say, runs directly counter to the idea of sets as classes that are composed of their members, and therefore it fails to explain in what sense NF is to be viewed as a theory of sets. It does not run counter to the idea of NF-"sets" as concept-correlates, on the other hand, and in fact, given the assumption that every object is a concept-correlate, it conforms perfectly to our generalized form of Frege's double correlation thesis for unsaturated higher level (unary) concepts. In this regard, we maintain, it is more appropriate to identify NF- "sets" with the concept-correlates of monadic $\lambda \mathrm{HST}^{*}+\left(\mathrm{Ext}^{*}\right)+\left(\mathrm{Q}^{*}\right)$ as a reconstruction of Frege's double correlation thesis than to construe NF as a first order reconstruction of the theory of types as a theory of classes.

\section{On Taking Urelements Seriously}

The idea that every object is a concept-correlate, i.e., Quine's thesis, is really not very plausible, and of course we do not recommend its adoption here. But since $\left(\mathrm{Q}^{*}\right)$ is equivalent to (Ext) in our reconstructed Fregean system, then dropping $\left(\mathrm{Q}^{*}\right)$ as an assumption means dropping $(E x t)$ as well. Note, however, than even without $\left(Q^{*}\right)$ the following restricted form of $(\mathrm{Ext})$ is provable in $\lambda \mathrm{HST}^{*}+\left(\mathrm{Ext}^{*}\right)$ :

$$
\forall x \forall y(\exists z(z \in x) \& \forall z[z \in x \leftrightarrow z \in y] \rightarrow x=y) .
$$

Now the result of modifying NF by replacing (Ext) by $\left(E^{\prime} t^{\prime}\right)$ is precisely the system NFU (New Foundations with Urelements) described by Jensen in "On the Consistency of a Slight(?) Modification of Quine's New Foundations," ([OCSM]). In that regard, accordingly, what the above observation shows is that NFU is contained in $\lambda \mathrm{HST}^{*}+\left(\mathrm{Ext}^{*}\right)$ in the same sense in which NF is contained in $\lambda \mathrm{HST}^{*}+\left(\mathrm{Ext}^{*}\right)+\left(\mathrm{Q}^{*}\right)$. The question then arises as to whether monadic $\lambda \mathrm{HST}^{*}+\left(\mathrm{Ext}^{*}\right)$ can also be shown to be consistent relative to NFU. 
The answer is affirmative, but in our proof we shall proceed through an intermediary system NFU', which is obtained from NFU by supplementing it with an individual constant $\wedge$ for the empty class. This means adding the axiom,

$$
\sim \exists x(x \in \wedge),
$$

and modifying the stratified comprehension principle of NFU as follows: (CP-NFU') $\exists y([\exists x(x \in y) \vee y=\wedge] \& \forall x[x \in y \leftrightarrow \phi])$,

where $\phi$ is a stratified first order wff of NFU' in which $y$ does not occur free. This modification of NFU is needed since in $\lambda \mathrm{HST}^{*}+\left(\mathrm{Ext}^{*}\right)$ there is a unique empty class (as a concept-correlate), whereas in NFU, because of the switch from (Ext) to $\left(E x t^{\prime}\right)$, there may well be more than one empty class. Of course, since (CP-NFU') implies the original comprehension principle of NF and NFU, then it follows that NFU is contained in NFU'. To prove that $\mathrm{NFU}^{\prime}$ can be translated into NFU, on the other hand, we consider the following function defined recursively on the first order wffs of NFU':

$$
\begin{aligned}
& t(x \in y)=(x \in y), \\
& t(x \in \wedge)=(x \neq x), \\
& t(\wedge \in x)=\exists y[\sim \exists z(z \in y) \& y \in x], \\
& t(x=y)=(x=y), \\
& t(x=\wedge)=t(\wedge=x)=\sim \exists z(z \in x), \\
& t(\sim \phi)=\sim t(\phi), \\
& t(\phi \rightarrow \psi)=[t(\phi) \rightarrow t(\psi)], \\
& t(\forall x \phi)=\forall x t(\phi) .
\end{aligned}
$$

Note that the $t$-transform of any instance of (CP-NFU') is a wff of NFU of the form:

$$
\exists y([\exists x(x \in y) v \sim \exists x(x \in y)] \& \forall x[x \in y \leftrightarrow \phi]),
$$

where $\phi$ is a stratified wff in which $y$ does not occur free, and therefore $t$ translates instances of (CP-NFU') into wffs that are trivially equivalent to 
the comprehension principle of NFU. But then since $t\left(\mathrm{Ext}^{\prime}\right)=\left(\mathrm{Ext}^{\prime}\right)$ and $t(\sim \exists x(x \in \wedge))=\sim \exists x(x \neq x)$, it follows that every theorem of NFU' is translated by $t$ into a theorem of NFU. In other words, NFU' is equiconsistent with NFU.

To show that monadic $\lambda \mathrm{HST}^{*}+\left(\mathrm{Ext}^{*}\right)$ is consistent relative to $\mathrm{NFU}^{\prime}$, we return to the translation function $s$ defined in section 5, except that clause (6) in the definition of $s$ is revised as follows:

$$
\begin{aligned}
& s(\forall x \phi)=\forall \bar{x} s(\phi) \\
& s(\forall F \phi)=\forall \bar{F}[\exists z(z \in \bar{F}) \vee \bar{F}=\wedge \rightarrow s(\phi)] .
\end{aligned}
$$

Needless to say, the proof that $s$ translates every theorem of monadic $\lambda \mathrm{HST}^{*}+\left(\mathrm{Ext}^{*}\right)$ into a theorem of NFU' is the same as our earlier proof regarding the translation of monadic $\lambda \mathrm{HST}^{*}+\left(\mathrm{Ext}^{*}\right)+\left(\mathrm{Q}^{*}\right)$ into NF. It follows, of course, that the product of the translations $t$ and $s$ (i.e., $t \cdot s$ ) translates every theorem of monadic $\lambda \mathrm{HST}^{*}+\left(\mathrm{Ext}^{*}\right)$ into a theorem of NFU. Accordingly, since for reasons already indicated we are able to drop the restriction to monadic $\lambda \mathrm{HST}^{*}+\left(\mathrm{Ext}^{*}\right)$, we have now proved the following metatheorem.

METATHEOREM 2. $\lambda \mathrm{HST}^{*}+\left(\right.$ Ext $\left.^{*}\right)$ is equiconsistent with NFU.

Now aside from the above result, what makes NFU particularly interesting here are certain results of Jensen regarding both the consistency of NFU and the status of the axioms of infinity and choice in NFU. In particular, Jensen has shown that the axiom of infinity (Inf) is not provable in NFU, and that therefore the axiom of choice (AC) is not refutable therein. (Specker's proof that $(\mathrm{AC})$ is disprovable in NF makes heavy use of (Ext).) This means that NFU is essentially weaker than NF, and therefore that $\left(\mathrm{Q}^{*}\right)$ in particular is not provable in $\lambda \mathrm{HST}^{*}+\left(\mathrm{Ext}^{*}\right)$. In addition, by the translation functions already defined, it follows that NFU + $(\operatorname{Inf})+(\mathrm{AC})$ is equiconsistent with $\lambda \mathrm{HS}^{*}+\left(\mathrm{Ext}^{*}\right)+\left(\operatorname{Inf}^{*}\right)+\left(\mathrm{AC}^{*}\right)$ (cf. [THST], sections 5 and 8).

Another important result that Jensen has established is that NFU ( + $(\operatorname{Inf}),+(\operatorname{Inf})+(\mathrm{AC}))$ is consistent if weak Zermelo set theory $(+(\operatorname{Inf})$, $+(\operatorname{Inf})+(\mathrm{AC}))$ is consistent. Weak Zermelo set theory (or what we shall call weak $\mathrm{Z}$ ) is the restriction of Zermelo set theory (without (Inf)) to 
those instances of the Aussonderungsaxiom in which all quantifiers in the comprehension clause are limited or restricted; i.e., in which all quantifiers have the form $(\forall x \in y) \phi$ or $(\exists x \in y) \phi$. It should be noted, however, that Jensen's proof is semantical; that is, it does not transform a proof in NFU into a proof in weak $Z$ but rather constructs a model of NFU in terms of a model of weak $Z$. Nevertheless, weak $Z$ is particularly interesting here since Jensen has also shown (again by a semantical proof) that weak $\mathrm{Z}(+(\operatorname{Inf}),+(\operatorname{Inf})+(\mathrm{AC}))$ is equiconsistent with the theory of simple types (or ST) as a theory of classes (plus an axiom of infinity or axioms of infinity and choice in type-theoretical terms).

In [THST], however, by using a Fregean semantics for $\lambda \mathrm{HST}^{*}+\left(\right.$ Ext $\left.^{*}\right)$ (in which general models are supplemented with a function correlating objects in the domain of discourse with values of the predicate variables), it was shown that a Fregean model of $\lambda \mathrm{HST}^{*}+\left(\mathrm{Ext}^{*}\right)\left(+\left(\operatorname{Inf}^{*}\right),+\left(\operatorname{Inf}^{*}\right)\right.$ $\left.+\left(\mathrm{AC}^{*}\right)\right)$ can be reconstructed into a general model of ST (with corresponding type-theoretical axioms of infinity and choice). Accordingly, where $A$ and $B$ are arbitrary formal systems and $(A \Rightarrow B)$ means that a (general) model of $B$ can be constructed in terms of a (general) model of $A$, and $(A \rightarrow B)$ means that $B$ can be translated into $A$ so that a proof in $B$ can be transformed into a proof in $A$, we can represent the situation described above as follows (noting that the connections hold with (Inf) or (Inf) $+(\mathrm{AC})$ and their counterparts as well).

$$
\begin{gathered}
\text { weak } \mathrm{Z} \Leftrightarrow \mathrm{ST} \\
\Downarrow \quad \uparrow \\
\mathrm{NFU} \leftrightarrow \lambda \mathrm{HST}^{*}+\left(\mathrm{Ext}^{*}\right)
\end{gathered}
$$

In other words, all of these formal systems are equiconsistent with one another.

METATHEOREM 3. Weak Z, ST, NFU and $\lambda \mathrm{HST}^{*}+\left(\mathrm{Ext}^{*}\right)$, with or without axioms of infinity and choice, are all equiconsistent with one another.

It should perhaps be emphasized before concluding this section, incidentally, that the equiconsistency between all of these systems does not show that the restricted form of the iterative concept of set (as represented by ST and weak Z) coincides with the logical concept of class (as 
represented by NFU and $\left.\lambda \mathrm{HST}^{*}+\left(\mathrm{Ext}^{*}\right)\right)$. Rather, the fact that some of the consistency proofs in question are semantical (and may involve general models at that) indicates that equiconsistency only shows that models of the one concept (as represented by one of the systems in question) can be constructed in terms of models of the other.

\section{An Alternative Modification of Frege's Double Correlation Thesis}

Now there is another way of modifying Frege's double correlation thesis so that Russell's paradox does not follow. In particular, instead of modifying the thesis as it applies directly to the positing of first level concepts and only indirectly to the positing of concept-correlates-i.e., instead of modifying the thesis as represented by the original comprehension principle (CP of concept-correlates. The framework for such a modification, incidentally, is implicit, at least in part, in Frege's discussion of Russell's paradox when he suggests that we might "suppose there are cases where an unexceptional concept has no class answering to it as its extension" ([BLA], 128). That is, instead of assuming that each singular term actually denotes (a value of the bound individual variables)-i.e., instead of assuming axiom (A3")-we might allow that some singular terms, and certain nominalized predicates in particular, are denotationless. The modification in question, in other words, is to be formulated in a logic that is free of existential presuppositions regarding singular terms.

Let us return, accordingly, to the second order logic with nominalized predicates formulated in section 3, i.e., the logic which we took as a counterpart to Frege's own original system. $\lambda$-abstracts need not be homogeneously stratified in this logic, it will be remembered, and although this returns us once again to Russell's paradox, a contradiction is avoided by adopting the suggestion in question, i.e., by allowing for denotationless singular terms. We may do so, moreover, first by replacing (A3*) by the following weaker, but clearly unexceptional, "law of logic":

$$
\left(\mathrm{A} 3^{* *}\right) \quad \forall x \exists y(x=y) \text {. }
$$

Secondly, since without (A3*) the identity law $(a=a)$, where $a$ is an arbitrary singular term, is no longer derivable, we shall have to add $(a=a)$ as 
an axiom schema as well. Finally, we shall need to replace $(\lambda$-Conv*) by the presupposition free form of $\lambda$-conversion:

$$
\begin{aligned}
(\exists / \lambda \text {-Conv } *) \quad & {\left[\lambda x_{1} \ldots x_{n} \phi\right]\left(a_{1}, \ldots, a_{n}\right) \leftrightarrow \exists x_{1} \ldots \exists x_{n}\left(a_{1}=x_{1}\right.} \\
& \left.\& \ldots \& a_{n}=x_{n} \& \phi\right),
\end{aligned}
$$

where no $x_{i}$ is free in any $a$, for all $i, j$ such that $1 \leqslant i, j \leqslant n$.

Now with the replacement of $\left(\mathrm{A} 3^{*}\right)$ by $\left(\mathrm{A} 33^{*}\right)$ and $(a=a)$, and of $(\lambda$ Conv*) by $(\exists / \lambda$-Conv*), the principle (UI:*) of universal instantiation of a singular term for an individual variable is no longer derivable except in the following qualified form:

$\left(\exists / \mathrm{UI}_{i}^{*}\right) \quad \exists y(a=y) \rightarrow[\forall x \phi \rightarrow \phi(a / x)]$,

where $a$ is any singular term that is free for $x$ in $\phi$ and in which $y$ has no free occurrences. Note that with this qualification what follows from Russell's paradox is not a contradiction but only that the predicate $[\lambda x \exists G(x=G \& \sim G(x))]$ is denotationless in its occurrences as a singular term; i.e., instead of a contradiction, only

$$
\sim \exists y([\lambda x \exists G(x=G \& \sim G(x))]=y)
$$

is provable. In other words, even though the unqualified comprehension principle $\left(\mathrm{CP}_{\lambda}^{*}\right)$ posits the existence of an unsaturated concept corresponding to $[\lambda x \exists G(x=G \& \sim G(x))]$ as a predicate, nevertheless by Russcll's argument it is provable in the system in question that there can be no saturated object corresponding to $[\lambda x \exists G(x=G \& \sim G(x))]$ as a singular term; or in Frege's words, here is a case "where an unexceptional concept has no class answering to it as its extension" (ibid.).

That some nominalized predicates must be denotationless in the present system, incidentally, does not show that all must be denotationless (cf. chapter 2, section 13 for a discussion of the latter option). Indeed, if arithmetic is to be derivable in the framework in cuestion, then we must assume that at least some concepts have concept-correlates. Of course, such an assumption can easily be given in terms of the existence conditions for sets in one or another version of set theory; but the result, while perhaps interesting as a second order set theory, will mean giving up the logical concept of class. That is, since the existence conditions in question are in accordance with the iterative concept of set, and therefore with the limitation or size doctrine, the resulting framework would really only be 
concerned with classes as composed of their members and not with classes in the logical sense. Note, incidentally, that it would be philosophically incoherent to drop the axiom of extensionality in such a second order set theory (or even in a first order set theory for that matter!) and call the result $a$ theory of properties (as abstract individuals). For even without an axiom of extensionality, the abstract individuals characterized by set-theoretical axioms are still posited in accordance with the iterative concept of set, and in that sense they are still composed of their members. Thus, if the objects denoted by nominalized predicates are to be "logical objects", which in the presence of an axiom of extensionality means that they are to be classes in the logical sense, then the relevant assumption regarding the positing of such objects should be in accordance with Frege's double correlation thesis and not with the iterative concept of set.

Now in regard to Frege's double correlation thesis, let us note that since we are retaining the full unrestricted comprehension principle $\left(\mathrm{CP}_{\hat{\lambda}}^{*}\right)$, then we are in effect also retaining the unrestricted form of Frege's double correlation thesis insofar as it applies to the positing of first level concepts. That is, we are retaining that part of the thesis that posits first level concepts corresponding to unequal leveled or inhomogeneous higher level relations no less so than to homogeneous or equal leveled relations. Thus, corresponding to Frege's unequal second level relation of subsumption or predication, there is in the system as so far described a first level relation of subsumption posited by $\left(\mathrm{CP}_{\mathrm{\lambda}}^{*}\right)$; i.e.,

$$
\exists R^{2}([\lambda x y \exists G(x=G \& G(y))]=R)
$$

is provable in the system in question. Of course, by Russell's argument this same predicate when nominalized must be denotationless; i.e., in its occurrences as a singular term it must fail to denote (a value of the bound individual variables). Or, in other words, as applied to concept-correlates, Frege's double correlation thesis must be restricted, and in particular it is not to apply to all of the first level concepts corresponding to or otherwise involving inhomogeneous higher level relations. The assumption we shall make here is that it is to apply at least (but by no means therefore only) to all of the first level concepts corresponding to higher level unsaturated concepts (including relations) that are homogeneously stratified; i.e., at least to all of the concepts that have concept-correlates in $\lambda \mathrm{HST}^{*}$. 
In order to formulate our assumption as an axiom schema, we shall say that a meaningful expression $\xi$ (as defined in terms of the logical grammar of section 3 ) is bound to individuals if and only if for all predicate variables $F$, and all wffs $\phi$, if $\forall F \phi$ is a wff occurring in $\xi$, then for some individual variable $x$ and some wff $\psi, \phi$ is the wff $[\exists x(F=x) \rightarrow \psi]$. Thus, to be bound to individuals, every predicate quantifier occuring in $\xi$ must refer only to those first level concepts posited by ( $\left.\mathrm{CP}_{\lambda}^{*}\right)$ that have corresponding concept-correlates. Our assumption regarding the existence of concept-correlates may now be formulated as the following axiom schema:

$(\exists / \mathrm{HSCP} \ddot{x}) \quad \exists y\left(a_{1}=y\right) \& \ldots \& \exists y\left(a_{k}=y\right) \rightarrow \exists y\left(\left[\lambda x_{1} \ldots x_{n} \phi\right]=y\right)$,

where $\left[\lambda x_{1} \ldots x_{n} \phi\right]$ is homogeneously stratified, $\phi$ is bound to individuals, $y$ is an individual variable not occuring in $\phi$, and $a_{1}, \ldots, a_{k}$ are all of the variables (or nonlogical constants) occurring free in $\left[\lambda x_{1} \ldots x_{n} \phi\right] .{ }^{10}$

The axiom schemas of our present system, accordingly, are (A0"), (A1*), (A2*), (A3*) $(a=a)$, where $a$ is any singular term, (LL*), (CP*), $\left(\exists / \lambda\right.$-Conv"), $\left(\operatorname{Id}_{\lambda}^{*}\right)$ and now $\left(\exists / \mathrm{HSCP}_{\lambda}^{*}\right)$ as well. ${ }^{11}$ (We retain modus ponens and universal generalization as our only inference rules.) Because of its relation to our earlier system $\lambda \mathrm{HST}^{*}$, we shall refer to this system hereafter as HST*.

Now it is clear that HST" is not a conservative extension of $\lambda \mathrm{HST}^{*}$, since whereas, by (A3*), $\forall F \exists x(F=x)$ is provable in $\lambda \mathrm{HST}^{*}$, this same wff, as witness the predicate $[\lambda x \exists G(x=G \& \sim G(x))]$, is actually disprovable in HST* Nevertheless, since every wff of $\lambda \mathrm{HST}^{*}$ is provably equivalent, again by (A3*), to a wff that is bound to individuals, HST* may be said to contain $\lambda \mathrm{HST}^{*}$ in the sense of the following lemma (which is easily seen to hold).

LEMMA. If $\phi$ is a wff of $\lambda \mathrm{HST}^{*}$ that is bound to individuals, $y$ is an individual variable not occurring in $\phi$, and $a_{1}, \ldots, a_{k}$ are all of the variables (or non-logical constants) occurring free in $\phi$, then $\overline{\mathrm{XHST}^{*}} \phi$ only if $\overline{\mathrm{HST}^{3}}$ $\exists y\left(a_{1}=y\right) \& \ldots \& \exists y\left(a_{k}=y\right) \rightarrow \phi$.

Now since an object that is denoted by a $\lambda$-abstract of $\lambda$ HST* $(+$ $\left(\mathrm{Ext}^{*}\right),+\left(\mathrm{Ext}^{*}\right)+\left(\mathrm{Q}^{*}\right)$, etc. $)$ is, by $\left(\mathrm{A} 3^{*}\right)$, also denoted by a $\lambda$-abstract 
that is bound to individuals, it follows, by $\left(\exists / \mathrm{HSCP}_{\lambda}^{*}\right)$, that every object that is a concept-correlate in $\lambda \mathrm{HST}^{*}\left(+\left(\mathrm{Ext}^{* *}\right),+\left(\mathrm{Ext}^{* *}\right)+\left(\mathrm{Q}^{*}\right)\right.$, etc. $)$ is also a concept-correlate in HST ${ }_{\lambda}^{*}\left(+\left(E^{*} t^{*}\right),+\left(E^{*} t^{* *}\right)+\left(Q^{*}\right)\right.$, etc. $)$; and therefore since NFU and NF are contained, respectively, in $\lambda \mathrm{HST}^{*}+$ $\left(\mathrm{Ext}^{*}\right)$ and $\lambda \mathrm{HST}^{*}+\left(\mathrm{Ext}^{*}\right)+\left(\mathrm{Q}^{*}\right)$, then NFU and NF are also contained (in the sense of the above lemma) in HST ${ }_{\lambda}^{*}+\left(\right.$ Ext $\left.^{*}\right)$ and HST ${ }^{*}+$ $\left(\mathrm{Ext}^{*}\right)+\left(\mathrm{Q}^{*}\right)$, respectively. Moreover, restricting ourselves to pure wffs (i.e., wffs in which no predicate or individual constants occur), it follows by the above lemma that every sentence (wff with no free variables) of $\lambda \mathrm{HST}^{*}$ that is bound to individuals and provable in $\lambda \mathrm{HST}^{*}\left(+\left(\mathrm{Ext}^{*}\right),+\right.$ $\left(\operatorname{Ext}^{* *}\right)+\left(Q^{*}\right)$, etc. $)$ is also provable in HST $\mathrm{X}_{\lambda}^{*}\left(+\left(\mathrm{Ext}^{*}\right),+\left(\mathrm{Ext}^{*}\right)+\right.$ $\left(Q^{*}\right)$, etc.) as well; i.e. that proofs in $\lambda$ HST $^{*}$ and its extensions can be transformed into related proofs in HST $\lambda_{\lambda}^{*}$ and its corresponding extensions. It follows, accordingly, that if $\operatorname{HST}_{\lambda}^{*}\left(+\left(\operatorname{Ext}^{* *}\right),+\left(\mathrm{Ext}^{* *}\right)+\left(\mathrm{Q}^{*}\right)\right.$, etc.) is consistent, then so is $\lambda \operatorname{HST}^{*}\left(+\left(\mathrm{Ext}^{* *}\right),+\left(\mathrm{Ext}^{*}\right)+\left(\mathrm{Q}^{*}\right)\right.$, etc. $)$.

It is also true, moreover, that if $\lambda \mathrm{HST}^{* *}+\left(\mathrm{Ext}^{* *}\right)$ or any of its extensions is consistent, then so is HST $\mathrm{H}_{\lambda}^{*}+\left(\mathrm{Ext}^{*}\right)$ and its corresponding extensions. The proof of this claim, however, is semantical and does not provide a method whereby a proof in HST ${ }_{\lambda}^{*}+\left(\right.$ Ext $\left.^{*}\right)$ of a wff of $\lambda \mathrm{HST}^{*}$ can be transformed into a proof in $\lambda \mathrm{HST}^{*}+\left(\mathrm{Ext}^{*}\right)$. (Cf. [FST], 169, n. 1, for the related claim regarding the impossibility of transforming proofs in ML into proofs in NF.) That is, the proof depends on developing a Fregean semantics for these systems that we shall not go into here (but cf. [LI], chapter 6, section 4, or the second paper listed in [THST], for the details of such a semantic proof). Nevertheless, on the basis of the details of that semantical proof it can be shown that a sentence of $\lambda \mathrm{HST}^{*}$ that is bound to individuals is provable in $\lambda \mathrm{HST}^{*}+\left(\mathrm{Ext}^{*}\right)$ if, as well as only if, it is provable in HST ${ }^{*}+\left(\right.$ Ext $\left.^{*}\right)$; and therefore it follows that the one system (and any of its extensions) is consistent if and only if the other (and its corresponding extensions) is consistent. Putting this result together with those already noted in the preceding sections, we have the following metatheorem.

METATHEOREM 4. HST* ${ }_{\lambda}^{*}+\left(\mathrm{Ext}^{*}\right)$, with or without axioms of infinity and choice, is equiconsistent with $\lambda \mathrm{HST}^{*}+\left(\mathrm{Ext}^{*}\right)$, weak Z, ST and NFU, all with or without corresponding axioms of infinity and choice; and $\mathrm{HST}_{\lambda}^{*}+\left(\mathrm{Ext}^{*}\right)+\left(\mathrm{Q}^{*}\right)$ is equiconsistent with NF. 


\section{Ultimate Classes and the Similarity of ML with HST $T_{\lambda}^{+}$} $\left(E x t^{*}\right)+\left(Q^{*}\right)$

Having given a natural motivation for both NF and NFU in terms of our first modification of Frege's double correlation thesis, let us now see how our second or alternative modification of Frege's thesis can be used to explain ML as well. In ML, it will be remembered, sets are not just classes that are composed of their members; rather, they are also classes that are elements. That is, in ML, which is also an applied first order theory with $\epsilon$ as its only primitive predicate constant, the notion of a set is defined as follows:

$$
\operatorname{Set}(x)=\mathrm{df} \exists z(x \in z) .
$$

Classes that are not sets are then referred to as proper or ultimate classes. (We shall follow Quine in Set Theory and Its Logic ([STL]), and call them ultimate classes hereafter.)

Of course, both the sets and ultimate classes of ML are assumed by Quine to be classes as composed of their members, which on Quine's view only means that the axiom of extensionality, (Ext), holds for both. In other words, according to Quine, the being of an ultimate class is no different from that of a class that is an element; they are both classes as composed of their members. But then why can't ultimate classes be elements? That a contradiction, viz., Russell's paradox, would ensue otherwise surely cannot be taken as an explanation of why ultimate classes can't be members of, say, finite classes (cf. [FST], 142).

The answer, we maintain, is that ultimate classes are really not objects to begin with, but are rather unsaturated concepts that have no classes answering to them as their extensions. That is, not being objects to begin with, we can hardly demand an explanation of why ultimate classes cannot be elements, for only an object can be a member, be it of a finite or an infinite class. The "sets" of ML, accordingly, are rcally not sets in the sense of classes that are composed of their members but are rather classes in the logical sense; i.e., they are classes that have their being as conceptcorrelates, and in particular they are the concept-correlates of monadic HST $\ddot{x}+\left(\right.$ Ext $\left.^{*}\right)+\left(Q^{* \prime}\right)$.

To see that this is so, let us note that by $\left(\exists / \lambda\right.$-Conv" $\left.{ }^{*}\right)$,

$$
\overline{\mathrm{HST}}[\lambda x F(x)](a) \rightarrow \exists x(a=x),
$$


where $x$ is not free in $a$; and therefore by ( $\left.\operatorname{Id}_{\lambda}^{*}\right)$ and (UG),

$$
\overline{\text { HST }^{*}} \forall F[F(a) \rightarrow \exists x(a=x)] .
$$

In other words, according to HST $\mathrm{T}_{\lambda}^{*}$, only real objects (in the sense of the values of the bound individual variables) can fall under concepts (which is not the same as to say that wffs containing denotationless singular terms cannot be true). Accordingly, assuming the same analysis of membership as given in section 5, it follows that only real objects can be elements:

$$
\widehat{\mathrm{HST}_{\mathrm{X}}} \exists x(a \in x) \rightarrow \exists x(a=x) .
$$

The converse also holds, moreover; for even though $(a=a)$ is true when $a$ is denotationless, nevertheless by $\left(\exists / \lambda\right.$-Conv $\left.{ }^{*}\right)$,

$$
\overline{\mathrm{HST}^{x}} \exists x(a=x \& x=x) \rightarrow[\lambda x(x=x)](a),
$$

and therefore since, by $\left(\mathrm{CP}_{\lambda}^{*}\right),[\lambda x(x=x)]$ stands for a concept, it follows by the definition of membership that

$$
\overline{\text { HST年 }} \exists x(a=x) \rightarrow \exists x(a \in x) .
$$

That is, according to HST ${ }_{\lambda}^{*}$, only elements can be real objects.

Now the ultimate classes of ML are really not objects, we have said, but are rather unsaturated concepts-or that in any case is how they should be viewed if we are to explain or provide a natural motivation for the classes of ML. Of course, in ML ultimate classes are represented as if they were real objects, since they are there taken as values of the bound individual variables. But that only means that the individual variables of ML should be reconstrued as 1-place predicate variables, so that all of the "classes" of ML, ultimate or otherwise, can be seen for what they are, viz., as unsaturated concepts; and then, only if they are also "elements" in ML, should they be viewed as having objects, viz., concept-correlates, corresponding to them. It is only in this way, we maintain, that we can explain why ultimate "classes" can't be elements, as well as provide a natural motivation for the classes of ML that are elements. In other words, where $q(\psi)$, for each wff $\psi$ of ML, is the result of replacing each individual variable in $\psi$ by a 1 -place predicate variable (where distinct individual variables are replaced by distinct predicate variables), our claim is that 
$q(\psi)$ is a theorem of monadic HST ${ }_{\lambda}^{*}+\left(\operatorname{Ext}^{*}\right)+\left(\mathrm{Q}^{*}\right)$ whenever $\psi$ is a theorem of ML.

Note in this regard that where $\psi$ is an instance of the comprehension principle *202 of ML for classes, ultimate or otherwise, then $q(\psi)$ is of the following form:

$$
\exists F \forall G(G \in F \leftrightarrow \exists H(G \in H) \& \phi) .
$$

In HST ${ }_{\lambda}^{*}$, however, not only does $\left(\mathrm{CP}^{*}\right)$ follow from $\left(\mathrm{CP}_{\lambda}^{*}\right)$, but, given our analysis of membership and the above observation that all and only real objects are elements, we also have the following:

$$
\text { HST } \exists F \forall x(x \in F \leftrightarrow \exists z(x \in z) \& \phi) \text {. }
$$

Therefore, by $\left(\mathrm{Q}^{*}\right)$, the $q$-transform of each instance of the comprehension principle ${ }^{*} 202$ of $\mathrm{ML}$ is a theorem of monadic HST $\mathrm{T}_{\hat{\lambda}}^{*}+\left(\mathrm{Q}^{*}\right)$.

Now aside from (Ext), the $q$-transform of which is easily seen to be derivable in $\mathrm{HST}_{\hat{\lambda}}^{*}$ on the basis of $\left(\mathrm{Ext}^{*}\right)$ and $\left(\mathrm{Q}^{*}\right)$, the remaining axiom schema of ML is *200, which depends on the notion of normalcy. ${ }^{12}$ In particular, a wff $\psi$ of ML is said to be normal if all of the bound variables in $\psi$ are restricted to elements; i.e., if in all of the subwffs of $\psi$ of the form $\forall x \phi, \phi$ is of the form $[\exists y(x \in y) \rightarrow \chi]$. Axiom schema * 200 can then be stated as follows (cf. Quine, Mathematical Logic, ([ML]), 162):

"200: If $\psi$ is a stratified, normal wff of ML, $y$ is new to $\psi$, and $x$, $z_{1}, \ldots, z_{n}$ are all of the distinct individual variables occurring free in $\psi$, then:

$$
\begin{aligned}
& \widehat{\mathrm{ML}} \exists y\left(z_{1} \in y\right) \& \ldots \& \exists y\left(z_{n} \in y\right) \rightarrow \exists y(\exists z(y \in z) \\
& \& \forall x[x \in y \leftrightarrow \exists z(x \in z) \& \psi]) .
\end{aligned}
$$

Needless to say, but by our above observation that all and only real objects are elements in HST ${ }_{\lambda}^{*}$, it is clear that a wff $\psi$ of ML is normal if and only if $q(\psi)$ is provably equivalent in HST $\mathrm{H}_{\mathrm{\lambda}}^{*}$ to a wff that is bound to individuals. Applying the same observation to $\left(\exists / \mathrm{HSCP}_{\hat{\lambda}}^{*}\right)$, moreover, we have the following result:

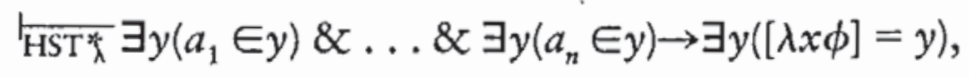

where $y$ is new to $\phi, x, a_{1}, \ldots, a_{n}$ are all the distinct variables occurring free in $\phi, \phi$ is bound to individuals and $[\lambda x \phi]$ is homogeneously strati- 
fied. But, again by the same observation, and by the same sort of argument that shows that $\left(\mathrm{CP}^{*}\right)$ is a consequence of $(\mathrm{CP} \ddot{\mathrm{\lambda}})$, the following is easily seen to hold:

$$
\begin{gathered}
\overbrace{\mathrm{HST} \cdot \hat{x}} \exists y([\lambda x \phi]=y) \rightarrow \exists y(\exists z(y \in z) \& \\
\& \forall x[x \in y \leftrightarrow \exists z(x \in z) \& \phi]) .
\end{gathered}
$$

Accordingly, by putting these results together and then applying $\left(\mathrm{Q}^{*}\right)$, it follows that the $q$-transform of every instance of $* 200$ is a theorem of monadic HST* $+\left(\mathrm{Ext}^{*}\right)+\left(\mathrm{Q}^{*}\right)$; and this completes our proof that the $q$ transform of a theorem of ML is a theorem of monadic HST* ${ }_{\lambda}^{*}+\left(\mathrm{Ext}^{*}\right)+$ $\left(Q^{*}\right)$.

We can also show, as might well be expected by now, that every theorem of monadic HST ${ }_{x}^{*}+\left(\mathrm{Ext}^{* *}\right)+\left(\mathrm{Q}^{*}\right)$ can be translated into a theorem of ML. For this purpose, we need only return to the translation function $s$ defined in section 5, except that clause (6) is revised as follows:

$$
\begin{aligned}
& s(\forall x \phi)=\forall \bar{x}[\exists z(\bar{x} \in z) \rightarrow s(\phi)], \\
& s(\forall F \phi)=\forall \bar{F} s(\phi) .
\end{aligned}
$$

The proof that $s$ translates each axiom of monadic HST ${ }_{\lambda}^{*}+\left(\mathrm{Ext}^{*}\right)+$ $\left(\mathrm{Q}^{*}\right)$ into a theorem of $\mathrm{ML}$ is similar to the proof that $s$ as originally defined in section 5 translates the axioms of monadic $\lambda \mathrm{HST}^{*}+\left(\mathrm{Ext}^{*}\right)+$ $\left(Q^{*}\right)$ into theorems of NF, except that now the element conditions that are part of the abbreviations (D1)-(D3) are essential to the proof. Putting these two results together, we can now state the following metatheorem (keeping in mind that monadic HST ${ }_{\lambda}^{*}$ is equiconsistent with the full system).

\section{METATHEOREM 5}

(a) If $\psi$ is a theorem of $\mathrm{ML}$, then $q(\psi)$ is a theorem of $\mathrm{HST} *$ $\left(\operatorname{Ext}^{*}\right)+\left(Q^{*}\right)$;

(b) if $\phi$ is a theorem of monadic HST* $+\left(\operatorname{Ext}^{*}\right)+\left(Q^{*}\right)$, then $s(\phi)$ is a theorem of $\mathrm{ML}$;

(c) therefore HST* $+\left(\right.$ Ext $\left.^{* *}\right)+\left(Q^{*}\right)$ is equiconsistent with ML. 
Finally, let MLU' (ML with urelements) be the result of replacing (Ext) in $\mathrm{ML}$ by $\left(\mathrm{Ext}^{\prime}\right)$, adding $\wedge$ as an individual constant together with the axiom,

$$
\sim \exists x(x \in \wedge)
$$

and modifying the comprehension principle *202 of ML as follows:

$\left(\mathrm{CP}-\mathrm{MLU}^{\prime}\right) \quad \exists y([\exists x(x \in y) \mathrm{v} y=\wedge] \& \forall x[x \in y \leftrightarrow \exists z(x \in z) \& \phi])$.

Then, by revising clause (6b) above of the translation function $s$ as follows,

$$
s(\forall F \phi)=\forall \bar{F}[\exists z(z \in \bar{F}) \vee \bar{F}=\bigwedge \rightarrow s(\phi)],
$$

we can repeat the above argument and show that $s$ translates every theorem of HST* $+\left(\mathrm{Ext}^{*}\right)$ into a theorem of MLU'.

What cannot be shown, however, is that the theorems of MLU' can be translated into theorems of HST* $+\left(\mathrm{Ext}^{*}\right)$. For example, the $q$-transform of $\left(\mathrm{Ext}^{\prime}\right)$ is not a theorem of HST $\mathrm{X}^{*}+\left(\mathrm{Ext}^{*}\right)$, since without $\left(\mathrm{Q}^{*}\right)$, the conditional

$$
\forall H(H \in F \leftrightarrow H \in G) \rightarrow \forall x(x \in F \leftrightarrow x \in G)
$$

is not provable in HST* $+\left(\mathrm{Ext}^{*}\right)$; i.e., without $\left(\mathrm{Q}^{*}\right)$ neither $(\mathrm{Ext})$ nor the $q$-transform of $\left(\mathrm{Ext}^{\prime}\right)$ is provable in HST ${ }_{\lambda}^{*}$ on the basis of (Ext"*). On the other hand, if we drop the idea of correlating the individual variables of ML with the predicate variables of monadic HST ${ }_{\lambda}^{*}+\left(\mathrm{Ext}^{*}\right)$, then, however defined, the transform of (CP-MLU') will not be provable in HST $+\left(\right.$ Ext $\left.^{*}\right)$, since it will in that case posit the existence of a conceptcorrelate corresponding to any given concept, which of course is impossible if HST ${ }^{*}+\left(\right.$ Ext $\left.^{*}\right)$ is consistent. In addition, such an alternative would mean giving up the whole idea of explaining why ultimate classes cannot be elements. The fact, in other words, that MLU' cannot be translated into HST* $+\left(\right.$ Ext* $\left.^{*}\right)$ indicates that MLU' lacks a natural motivation, especially since, as already noted in section 7, every class in NFU turns out to be a concept-correlate in HST $+\left(\right.$ Ext $\left.^{*}\right)$. Accordingly, if we are to drop Quine's thesis, whether in the form (Ext) or $\left(Q^{*}\right)$, then the appropriate framework is not MLU' but HST ${ }^{*}+\left(E^{*} t^{*}\right)$ instead. 


\section{On Mathematical Induction and the Class of Fregean Natural Numbers}

Quine's principal reason for proposing ML, it should be noted, is that, unlike the situation in NF, mathematical induction can be proved in ML without restriction to stratified wffs (cf. [ML] 165; [STL], 300). This is because in both NF and ML it is Frege's definition of the natural numbers that is both natural and most appropriate (cf. [STL], 289); and, of course, that is as it should be if both NF and ML are to be explained in terms of Frege's double correlation thesis. On this definition, at least as reconstructed in both NF and ML, a natural number is any class that belongs to every class, ultimate or otherwise, to which 0 belongs and which is closed under the successor relation. By definition, accordingly, the only form of mathematical induction that can be proved in NF is one that is restricted to stratified wffs (even though not all of the wffs of NF are stratified), since classes in general are specified in NF only in terms of stratified wffs. In ML, however, stratification is not necessary for the specification of classes in general, but only for the specification of classes that are elements. Thus, given Frege's definition of the natural numbers, it is only in ML that the full form of mathematical induction is provable.

The problem, however, is that although the class of natural numbers is specifiable in terms of a stratified wff, and therefore can be proved to exist within NF, the same specification when given in ML proves the "existence" of the class of natural numbers only in the sense of its being a value of the bound individual variables, and not also in the more important sense (at least for the purposes of mathematics) of its being an element. Or, given our reconstrual of the individual variables of $M L$ as the predicate variables of monadic HST H $^{*}+\left(\right.$ Ext $\left.^{*}\right)+\left(Q^{*}\right)$, it is only the concept of a natural number whose existence (as a value of the bound predicate variables) is assured, and not also the class of natural numbers as an object that is correlated with this concept.

Of course, the class of natural numbers whose existence is proved in NF can be proved to be an element in ML; but, as specified in ML (i.e., in terms of a wff that is both stratified and normal), this class does not validate the full form of mathematical induction. Nevertheless, since the same specification without the normalcy condition is even more restrictive (i.e., the wff without the normalcy condition implies the wff with the 
normalcy condition), then the class as specified with the normalcy condition is really the class of natural numbers only if it is identical with the class that is specified without the normalcy condition; and therefore, since the one is an element in ML, we may assume that the "other" is as well (cf. [STL], 303f). In other words, according to Quine, we may assume as an additional axiom of ML the identity of the class of natural numbers as specified with the normalcy condition and as specified without this condition. In that way, one can prove in ML both the unrestricted form of mathematical induction and the elementhood of the class of natural numbers (as specified in terms of ultimate classes, i.e. without the normalcy condition).

There is an objection to adding such an axiom to ML, however; namely, that insofar as "ML was proposed as an improvement of NF because ML has full-fledged mathematical induction whereas NF has induction for stratified formulae only, one may prefer to stick to NF since, once one starts adjoining to ML axioms as mentioned, one might as well add an axiom of full mathematical induction to NF" ([FST], 171). Now it is important to note that such an objection, while it might apply to Quine's own reason for proposing ML (especially when one attempts to view both NF and ML merely as theories of classes as composed of their members), completely misses its mark when NF and ML are explained in terms of Frege's double correlation thesis; i.e. when NF is viewed as contained in $\lambda \mathrm{HST}^{*}+\left(\mathrm{Ext}^{*}\right)+\left(\mathrm{Q}^{*}\right)$ and $\mathrm{ML}$ is viewed (under the $q$ transformation) as contained in $\mathrm{HST}_{\lambda}^{*}+\left(\mathrm{Ext}^{*}\right)+\left(\mathrm{Q}^{*}\right)$. Of course, in that case, we might just as well drop (Q"), or equivalently, (Ext), as a form of Quine's thesis and replace NF and ML by their preferred Fregean counterparts, $\lambda \mathrm{HST}^{*}+\left(\mathrm{Ext}^{*}\right)$ and $\mathrm{HST}_{\lambda}^{*}+\left(\mathrm{Ext}^{*}\right)$.

In reconsidering the problem from this perspective, in other words, we must first understand that the reason for proposing $\mathrm{HST}_{\lambda}^{*}+\left(\mathrm{Ext}^{*}\right)$ as an alternative to $\lambda \mathrm{HST}^{*}+\left(\mathrm{Ext}^{*}\right)$ is not because it has the full form of mathematical induction as a consequence of Frege's definition of the natural numbers, but rather because it is an alternative to $\lambda \mathrm{HST}^{*}+\left(\mathrm{Ext}^{*}\right)$ as a reconstruction of Frege's double correlation thesis. Such an alternative, moreover, does not extend $\lambda \mathrm{HST}^{*}+\left(\mathrm{Ext}^{*}\right)$ by the introduction of a new notion, such as that of an ultimate class as opposed to the notion of a class as an element, since in both $\lambda \mathrm{HST}^{*}+\left(\mathrm{Ext}^{*}\right)$ and HST ${ }_{\lambda}^{*}+\left(\mathrm{Ext}^{*}\right)$ all classes are elements. Indeed, since the distinction between an ultimate 
class and a class that is an element is really that between a concept and a class, then, on our explanation no new notion has really been introduced in ML after all; for the distinction between concepts and the classes that are their extensions is as fundamental in $\lambda \mathrm{HST}^{*}+\left(\mathrm{Ext}^{*}\right)$ as it is in HST ${ }^{*}$ $+\left(\mathrm{Ext}^{*}\right)$.

Secondly, if HST ${ }_{\lambda}^{*}+\left(\right.$ Ext $\left.^{* *}\right)$ is to be preferred to $\lambda \mathrm{HST}^{*}+\left(\mathrm{Ext}^{* *}\right)$, then it is not so much because the unrestricted form of mathematical induction can be proved therein as because it leaves umodified the full force of Frege's double correlation thesis insofar as the latter applies to the positing of first level concepts. In other words, whereas according to $\lambda \mathrm{HST}^{*}+\left(\mathrm{Ext}^{*}\right)$ there are no higher level inhomogeneous relations, or at least none that can be correlated with the first level concepts posited in (HSCP'), the situation is completely otherwise in HST ${ }_{\lambda}^{*}+\left(\mathrm{Ext}^{*}\right)$; and in that regard there may well be more classes implicit in the latter framework than are acknowledged in the former, and therefore more than are posited in $\left(\exists / \mathrm{HSCP}_{\lambda}^{*}\right)$. Thus, while Russell's paradox shows us that not all of the first level concepts that correspond to or otherwise involve inhomogeneous higher level relations have a corresponding concept-correlate, nothing follows to the effect that none of these concepts can have such a corresponding concept-correlate. In particular, where $S$ abbreviates the $\lambda$-abstract that stands for Frege's successor relation (cf. [STL], 279), and

$$
\begin{aligned}
& 0=\mathrm{df}[\lambda x \exists F(x=F \& \sim \exists y F(y))], \\
& N=\mathrm{df}[\lambda x \forall F(F(0) \& \forall y \forall z[F(y) \& S(y, z) \rightarrow F(z)] \rightarrow F(x))],
\end{aligned}
$$

then even though $N$ is not bound to individuals (and therefore is not posited in $(\exists / \mathrm{HSCP} \times)$ as denoting a class), nevertheless, it seems plausible to assume

$$
\exists x(N=x)
$$

as an additional axiom. That is, it seems plausible to assume that as represented in HST ${ }_{\hat{\lambda}}^{*}+\left(\mathrm{Ext}^{*}\right)$ the Fregean concept of a natural number has a class answering to it as its extension. It follows of course that in HST $+\left(\mathrm{Ext}^{*}\right)+\left(\mathrm{N}^{*}\right)$ we have both the class of natural numbers as a concept-correlate and the unrestricted form of the principle of mathematical induction.

Note, incidentally, that where 
$N^{\prime}=\operatorname{df}[\lambda x \forall w(0 \in w \& \forall y \forall z[y \in w \& S(y, z) z \in w] \rightarrow x \in w)]$,

then although we can prove the following identity wff in HST $+\left(\mathrm{Ext}^{*}\right)$,

$$
\begin{aligned}
& N^{\prime}=[\lambda x \forall F(\exists w(F=w) \& F(0) \& \forall y \forall z[F(y) \& S(y, z) \rightarrow \\
& F(z)] \rightarrow F(x))],
\end{aligned}
$$

nevertheless we cannot prove $\left(N=N^{\prime}\right)$ in HST ${ }^{*}+\left(\right.$ Ext $\left.{ }^{*}\right)$ without assuming $\left(\mathrm{N}^{*}\right)$. In other words, since $\forall x\left[N(x) \rightarrow N^{\prime}(x)\right]$ is provable even in HST ${ }^{\prime}$, the class denoted by $N^{\prime}$ may contain objects other than the natural numbers as well; and if that is the case, then this alone would suffice as a reason to prefer HST ${ }^{*}+\left(\right.$ Ext $\left.^{*}\right)+\left(\mathrm{N}^{*}\right)$ over $\lambda$ HST $^{*}+\left(\right.$ Ext $\left.^{*}\right)$ even when the unrestricted principle of mathematical induction is added to the latter. For it is easily seen that $N=N^{\prime} \leftrightarrow\left(N^{*}\right)$ is provable in HST ${ }^{*}+$ $\left(\mathrm{Ext}^{*}\right)$.

Now we are not suggesting that $\left(N \neq N^{\prime}\right)$ is true in the framework represented by HST ${ }^{*}+\left(\mathrm{Ext}^{*}\right)$, but only that one appropriate way to investigate this question is by considering additional axioms or principles regarding the positing of concept-correlates for first level concepts that either correspond to or otherwise involve inhomogeneous higher level relations, and which might thereby warrant assuming $\left(N^{*}\right)$, from which $\left(N=N^{\prime}\right)$ follows in HST ${ }^{*}+\left(\mathrm{Ext}^{*}\right)$. The initial system HST not proposed as an alternative to $\lambda \mathrm{HST}^{*}$ because it allows for the unrestricted principle of mathematical induction, but rather because it captures the full force of Frege's double correlation thesis at least as far as the positing of first level concepts is concerned. The axiom ( $\exists / \mathrm{HSCP}$ ) then assures us that all of the concept-correlates (or classes, given (Ext*)) that can be proved to exist in $\lambda \mathrm{HST}^{*}$ are carried over into HST* but by no means does it exhaust all of the possibilities as to which concepts can or cannot have concept-correlates.

In concluding, it should perhaps be noted that without Quine's thesis, whether in the form $(E x t)$ or $\left(Q^{*}\right)$, the axiom of infinity is not provable in either $\lambda \mathrm{HST}^{*}+\left(\mathrm{Ext}^{*}\right)$ or HST ${ }^{*}+\left(\mathrm{Ext}^{*}\right)+\left(\mathrm{N}^{*}\right)$. Since such an axiom is needed at least for the purposes of logicism, it may be added to both of these systems in either one of the usual forms (cf. [THST], section 5) or as follows when relations are included as well: 
$($ Inf*) $\forall F^{n} \forall G^{n}(F \neq G)$, where $m, n$ are arbitrary natural numbers such that $m \neq n$.

In other words, if for all distinct natural numbers $m$ and $n$ the conceptcorrelate of any $m$-ary relation is other than the concept-correlate of any $n$-ary relation, then there are infinitely many (or at least a potential infinity) of concept-correlates. Such an assumption, it should be noted, is independent of the question of how many objects other than conceptcorrelates there are, and in particular of how many concrete objects there are.

\section{Notes}

1 In an intensional language, the mathematical notion of a class might well assume a stronger axiom of extensionality, viz. one in which classes that are composed of their members are necessarily identical when they have the same members. Such an axiom would not in general hold for classes in the logical sense, since co-extensive concepts are not in general necessarily co-extensive. (It would of course hold for those classes in the logical sense that are the extensions of "rigid" concepts, i.e., concepts that have the same extension in every possible world.)

2 We ignore the question here of whether or in what sense the axiom of replacement accords with the iterative concept of set (cf. [ICS], section 4). We could just as well speak of Zermelo set theory instead.

3 One can of course model variable binding operators in terms of sets (as is done in [SOL]). But in that case, at least if the operators are understood to stand for Frege's higher level concepts, the modeling must be restricted to Henkin's general models for type theory (cf. [C'ГГ]). To insist that general models are not the real or "primary" semantics for variable binding operators is to confuse Frege's theory of types (if one insists on calling it that) with Russell's-or what essentially comes to the same thing given Frege's extensional view of concepts, it is to confuse Frege's hierarchy of concepts with a restricted form of the iterative concept of set.

4 Frege's thesis is stated for all second level functions, not just for concepts, i.e., not just for those functions that have truth-values as their values. For convenience (and otherwise for reasons discussed in [FRL], section 2) we shall ignore functions other than concepts. Also, although we shall in general restrict ourselves to the monadic case throughout, we shall also sometimes refer to relations as concepts.

5 The rewrite law,

$$
\left[\lambda x_{1} \ldots x_{n} \phi\right]=\left[\lambda y _ { 1 } \ldots y _ { n } \phi \left(y_{1}\left(x_{1}, \ldots, y_{n}\left(x_{n}\right)\right],\right.\right.
$$

where no $y_{i}$ occurs in $\phi$, is derivable in $\lambda \mathrm{HST}^{*}$ on the basis of $\left(\mathrm{Ext}^{*}\right)$-or its intensional counterpart $\left(\square \mathrm{Ext} \mathrm{t}^{*}\right)$ described below. If neither of these principles are assumed, then 
the rewrite law must be taken as an additional axiom schema of $\lambda \mathrm{HST}^{*}$, or of $\square \lambda H S T^{*}$ as described below.

6 This notion occurs in Montague [FP], 132. It also occurs in [IHML], 77, where the principle of extensional comprehension is the type-theoretical counterpart of the principle of rigidity described below.

7 Although this last claim is correct as it stands, it would nevertheless be more natural to extend both the notion and the principle of rigidity to relations as well (cf. [FRL] section 13).

8 Cf. $[L I]$, chapter 6 , section 5 for a semantics of $\lambda H S T$ " that does not validate either (Ext") or (DExt $\left.{ }^{*}\right)$.

9 Since we want to both use and mention $\in$ here, we shall take it as an autonymous sign, i.e., as one that serves to name itself. The same observation applies to $=$.

10 We of course understand the "conditional" posited in $(\exists / \mathrm{HSCP} \lambda)$ to be just the consequent if $k=0$, i.e. if the $\lambda$-abstract in question contains no free variables (or nonlogical constants).

11 The rewrite law cited in note 5 is needed here as well if neither (Ext") nor ( $\square$ Ext $\left.{ }^{*}\right)$ is assumed as a supplementary axiom.

12 This notion of normalcy comes from Gödel $[\mathrm{CCH}], 12$. Its application to ML was first given in Wang [FSL].

\section{References}

[ICS] G. Boolos, "The Iterative Conception of Set," Journal of Philosophy 68 (1971):215-31.

[SA] A. Church, "Schröder's Anticipation of the Simple Theory of Types," Journal of Unified Science (Erkenntnis) 9 (1939):149-52.

[SOL] N. Cocchiarella, "A second order logic of variable-binding operators," Reports on Mathematical Logic 5 (1975):3-18.

[THST] N. Cocchiarella, "The Theory of Homogeneous Simple Types as a Second Order Logic," Notre Dame Journal of Formal Logic 20 (1979):505-24; and also "Two $\lambda$-Extensions of the Theory of Homogeneous Simple Types as a SecondOrder Logic," Notre Dame Journal of Formal Logic 26 (1985): 377-407.

[liRL] N. Cocchiarella, "Frege, Russell and Logicism: a Logical Reconstruction," in Frege Synthesized, L. Haaparanta and J. Hintikka, eds, D. Reidel, Dordrecht, 1986. Reprinted here as chapter 2.

[LI] N. Cocchiarella, Logical Investigations of Predication Theory and the Problem of Universals, vol. 2 of Indices (Naples: Bibliopolis Press, 1986).

$[B L A]$ G. Frege, The Basic Laws of Arithmetic, translated by M. Furth (Berkeley: University of California Press, 1964).

[PW] G. Frege, Posthumous Writings, eds. H. Hermes, F. Kambartel and F. Kaulbach; translated by P. Long and R. White (Oxford: Blackwell, 1979).

[GSB] G. Frege, Translations from the Philosophical Writing of Gottlob Frege, eds. P. Geach and M. Black (Oxford: Blackwell 1952). 
192 The Double Correlation Thesis and Set Theories NF and ML

[FST] A. Fraenkel, Y. Bar-Hillel, and A. Levy, Foundations of Set Theory (NorthHolland, Amsterdam, 1973).

[IHML] D. Gallin, Intensional and Higher-Order Modal Logic (North-Holland, Amsterdam, 1975).

[CCH] K. Gödel, The Consistency of the Continum Hypothesis (Princeton: Princeton University Press, 1940).

[CTT] L. Henkin, "Completeness in the Theory of Types," Joumal of Symbolic Logic 15 (1950):81-91.

[OCSM] R. Jensen, "On the Consistency of a Slight(?) Modification of Quine's New Foundations," Synthesé 19 (1968):250-63.

[TN] G. Kreisel, "Two Notes on the Foundations of Set-Theory," Dialectica 23 (1969):93-114.

[FP] R. M. Montague, Formal Philosophy, ed. R. H. Thomason (New Haven: Yale University Press, 1974).

$[M L]$ W. V. O. Quine, Mathematical Logic (Cambridge: Harvard University Press, 1958).

[STL] W. V. O. Quine, Set Theory and Its Logic (Cambridge: Harvard University Press, 1963).

[OSD] B. Russell, "On Some Difficulties in the Theory of Transfinite Numbers and Order Types," Proc. of London Math. Soc. (1906); reprinted in Essays in Analysis. ed. D. Lackey (New York: Braziller, 1973).

$[P M]$ B. Russell, and A. Whitehead, Principia Mathematica (Cambridge University Press, 1913).

[AOC] E. Specker, "The Axiom of Choice in Quine's New Foundations for Mathematical Logic," Proc. of the Nat. Acad. of Sciences 39 (1953), 972-75.

[TA] E. Specker, "Typical ambiguity," in Logic, Methodology and Philosophy of Science, eds. E. Nagel et al. (Stanford University Press, 1962):116-24.

[FSL] H. Wang, "A formal system of logic," Joumal of Symbolic Logic 15 (1950): $25-$ 32.

[FMP] H. Wang, From Mathematics to Philosophy New York; (Humanities Press 1974). 


\section{Russell's Theory of Logical Types and the Atomistic Hierarchy of Sentences}

Russell's philosophical views underwent a number of changes throughout his life, and it is not always well-appreciated that views he held at one time came later to be rejected; nor, similarly, that views he rejected at one time came later to be accepted. It is not wellknown, for example, that the theory of logical types Russell described in his later or post- $[P M]$ philosophy is not the same as the theory originally described in $[P M]$ in 1910-13; nor that some of the more important applications that Russell made of the theory at the earlier time cannot be validated or even significantly made in the framework of his later theory. What is somewhat surprising, however, is that Russell himself seems not to have realized that he was describing a new theory of logical types in his later philosophy, and that as a result of the change some of his earlier logical constructions, including especially his construction of the different kinds of numbers, were no longer available to him.

In the original framework, for example, propositional functions are independently real properties and relations that can themselves have properties and relations of a higher order/type, and all talk of classes, and thereby ultimately of numbers, can be reduced to extensional talk of properties and relations as "single entities," or what Russell in [POM] had called "logical subjects." The Platonic reality of classes and numbers was replaced in this way by a more fundamental Platonic reality of proposi- 
tional functions as properties and relations. In Russell's later philosophy, however, "a propositional function is nothing but an expression. It does not, by itself, represent anything. But it can form part of a sentence which does say something, true or false" (Russell, My Philosophical Development, ([MPD]), 69). Surprisingly. Russell even insists that this was what he meant by a propositional function in $[P M]$. "Whitehead and I thought of a propositional function as an expression containing an undetermined variable and becoming an ordinary sentence as soon as a value is assigned to the variable: ' $x$ is human', for example, becomes an ordinary sentence as soon as we substitute a proper name for ' $x$ '. In this view ... the propositional function is a method of making a bundle of such sentences" ([MPD], 124). Russell does realize that some sort of change has come about, however, for he admits, "I no longer think that the laws of logic are laws of things; on the contrary, I now regard them as purely linguistic" (ibid., 102).

How an uncountable reality of classes and numbers can be reduced to a countable reality of "linguistic conveniences," Russell never explained; but it is clear that he thought that such a reduction was already accomplished in $[P M]$, i.e., that $[P M]$ could sustain a nominalistic construal of propositional functions. Now whether or not $[P M]$ can sustain such an interpretation is not our concern here (though, given the axioms of reducibility and infinity, we think it cannot); for what Russell failed to see was that the theory of types he described and was committed to in his later philosophy was but a fragment of the theory described in $[P M]$, and that in fact the analysis of classes and numbers given in $[P M]$ cannot be given in this fragment. This new theory of types was dictated by what Russell later called "the technical form of the principle of atomicity"; namely, the thesis that "all propositions are either atomic, or molecular, or generalizations of molecular propositions; or at least, that a language of which this is true, and into which any statement is translatable, can be constructed" (Russell, An Inquiry into Meaning and Truth, ([Inq]), 250f). The "logical language" in question here is what Russell called "the atomistic hierarchy of sentences" (ibid., 187), and it amounts, as we shall see, to but a fragment of second order predicate logic. Russell does also allow for a hierarchy of "languages" constructed on the basis of the atomistic hierarchy, but this additional hierarchy, as we shall also see, turns out to be essen- 
tially a nominalistic construal of ramified second order logic. That is, ramified second order logic is all that is left in Russell's later philosophy of his original theory of types. This system is not only much weaker than Russell's original logic, but, even worse, on grammatical grounds alone it cannot sustain Russell's analysis of classes and numbers. For, despite Russell's misleading notation otherwise (in his 1925 introduction to $[P M]$ ), propositional functions (construed as expressions) cannot occur as (higher order) abstract singular terms in ramified second order logic, and yet it is precisely their occurrence as such that is essential to Russell's analysis of classes and numbers.

Now it is not whether $[P M]$ can sustain a nominalistic interpretation that is our concern in this essay, as we have said, but rather how it is that Russell came to be committed in his later philosophy to the atomistic hierarchy and the nominalistic interpretation of propositional functions as expressions generated in a ramified second order hierarchy of languages based on the atomistic hierarchy. We shall pursue this question by beginning with a discussion of the difference between Russell's 1908 theory of types and that presented in $[P M]$ in 1910 . This will be followed by a brief summary of the ontology that Russell took to be implicit in $[P M]$, and that he described in various publications between 1910 and 1913. The central notion in this initial discussion is what Russell in his early philosophy called the notion of a logical subject, or equivalently that of a "term" or "single entity". (In $[P M]$, this notion was redescribed as the systematically ambiguous notion of an "object.") As explained in chapter 1 this notion provides the key to the various problems that led Russell in his early philosophy to the development of his different theories of types, including that presented in $[P M]$. This remains true, moreover, even when we turn to Russell's later philosophy, i.e., to his post- $[P M]$ views, only then it is described as the notion of what can and cannot be named in a logically perfect language. The ontology of these later views is what Russell called logical atomism, and it is this ontology that determines what Russell described as the atomistic hierarchy of sentences. In other words, it is the notion of what can and cannot be named in the atomistic hierarchy that explains how Russell, however unwittingly, came to replace his earlier theory of logical types by the theory underlying the atomistic hierarchy of sentences as the basis of a logically perfect language. 


\section{The 1910 versus the 1908 Theory of Logical Types}

An important fact that is commonly overlooked in most of the literature on the theory of logical types is that the theory Russell described in [I'M] in 1910 is not the same as the theory he described in [1908] —unless, that is, one assumes that both propositions (as objective truths and falsehoods) and propositional functions are for Russell "single entities" in both theories. Russell did not assume this, however, and in fact while propositions are single entities in [1908], propositional functions, or so Russell then thought, were nonentities. Two years later, in $[P M]$, propositional functions are reckoned as single (nonlinguistic) entities, and propositions are reconstrued by Russell as not being single entities after all. The difference, apparently, was the result of Russell's shifting from a propositional theory of belief or judgment to his famous multiple relations theory (which he later rejected in 1913 as a result of criticisms by Wittgenstein). Thus, according to Russell in $[P M]$, "what we call 'a proposition' (in the sense in which this is distinguished from the phrase expressing it) is not a single entity at all," and in fact "the phrase which expresses a proposition is what we call an 'incomplete' symbol" (p. 44). We should note, incidentally, that being a single entity is what Russell also means by being capable of being a logical subject.

To see what this difference between the two theorics comes to, let us turn to Alonzo Church's formal characterization of Russell's ramified types, hereafter called r-types, and orders. ${ }^{1}$

There is an r-type $\mathrm{i}$ to which all and only individuals belong, and whose order is stipulated to be 0 .

If $\mathrm{m} \epsilon \omega, \mathrm{n} \epsilon \omega-\{0\}$, and $\beta_{1}, \ldots, \beta_{\mathrm{m}}$ are given $\mathrm{r}$-types, then there is an r-type $\left(\beta_{1}, \ldots, \beta_{\mathrm{m}}\right) / \mathrm{n}$ to which belong all and only m-ary propositional functions of level $\mathrm{n}$ and with arguments of r-types $\beta_{1}, \ldots, \beta_{\mathrm{m}}$, respectively; and the order of such a function is $N+\mathrm{n}$, where $\mathrm{N}$ is the greatest of the orders corresponding to the types $\beta_{1}, \ldots, \beta_{\mathrm{m}}$ (and $\mathrm{N}=0$ if $\mathrm{m}$ $=0$ ).

The notion of the level of a propositional function $\phi$ of $\mathrm{r}$-type $\left(\beta_{1}, \ldots\right.$ ,$\left.\beta_{m}\right) / \mathrm{n}$ is needed here, it should be noted, as a counterpart to Russell's 
nonsyntactical use (in 1910) of the notion of an apparent (or "bound") variable. Thus if $\mathrm{N}$ is the greatest of the orders corresponding to $\beta_{1}, \ldots$ ,$\beta_{\mathrm{m}}$, and $\mathrm{k}$ is the greatest of the orders of the apparent variables occurring in $\phi$ (in Russell's nonsyntactical sense), then $\mathrm{n}=1$ if $\mathrm{k} \leqslant \mathrm{N}$, and $\mathrm{n}=\mathrm{k}+$ 1 if $\mathrm{N}<\mathrm{k}$. Since $\phi$ is said to be predicative, according to Russell, when "it is of the lowest order compatible with its having the arguments it has" $([P M], 53)$, then in terms of the notion of level, if follows that $\phi$ is predicative if, and only if, $\mathrm{n}=1 .^{2}$

Now the above definition recognizes both propositions and propositional functions as single entities. Propositions of order $\mathrm{n}$, for example, are represented here as 0 -ary propositional functions of level $n$; i.e., as propositional functions of r-type ( $/ \mathrm{n}$, where ' () ' represents the null sequence. This of course is merely a convenience of terminology, since propositions are really not propositional functions in the intended sense. That both propositions and propositional functions are "single entities" is acknowledged here in the fact that both can occur as arguments of propositional functions, or as "logical subjects" of the resulting propositions. For example, an individual can stand to a propositional function of $\mathrm{r}$-type $\left(\beta_{1}, \ldots, \beta_{\mathrm{m}}\right) / \mathrm{n}$ in a predicative relation of r-type $\left(\mathrm{i},\left(\beta_{1}, \ldots, \beta_{\mathrm{m}}\right) / \mathrm{n}\right) /$ 1 ; and where belief is a predicative relation between an individual and a proposition of order $\mathrm{n}$, belief will be a propositional function of $\mathrm{r}$-type (i, ()$/ \mathrm{n}) / 1$.

Church is not unaware that Russell rejected propositions as single entities in 1910, and that he did so on the basis of his multiple relations theory of belief. Church claims, however, that the "fragmenting of propositions" that is involved in the multiple relations theory also requires the "fragmenting of propositional functions" (Church, "Comparison of Russell's Resolution of the Semantical Antinomies with that of Tarski," ([CRR]), 748), and therefore if propositional phrases are to be analyzed as incomplete symbols then phrases for propositional functions must also be so analyzable as well. The result would mean that the only category or type that was really fundamental with respect to quantification was that of the individuals, since only individuals would then remain as real single entities. The result, in other words, would mean that the theory of logical types was reducible to first order logic. Such a reduction was certainly not intended by Russell, and in any case, or so Church argues, "it is probable 
that the contextual definitions [i.e., analyses of phrases for propositions and propositional functions as incomplete symbols] would not stand scrutiny" (ibid.).

Actually, Church is not correct in thinking that the fragmenting of propositional functions that is involved in Russell's multiple relations theory means that propositional functions are ultimately to be eliminated as single entities. For although the propositional functions that occur in a belief or judgment on this theory are indeed "fragmented" in the sense of analysis, nevertheless each propositional function, as well as the "fragments" of that function that result upon analysis, retains its status as a single entity in the belief or judgment complex. Consider, for example, the judgment that all men are mortal as made by some person $\mathrm{A}$. The truth of this judgment, according to Russell, is a "second order truth" ([PM], 45), and that this is so can be seen in the following analysis:

$$
\text { Judges }(A,(x)[\hat{\phi} ! x \supset \hat{\psi} ! x], \hat{x} \text { is a man, } \hat{x} \text { is mortal). }
$$

We assume in this analysis that $\hat{x}$ is a man and $\hat{x}$ is mortal are predicative propositional functions of $\mathrm{r}$-type (i)/1 and that $(\mathrm{x})[\hat{\phi} ! \mathrm{x} \supset \hat{\psi} ! \mathrm{x}]$ is a propositional function of $r$-type $((\mathrm{i}) / 1,(\mathrm{i}) / 1) / 1$, and therefore of order 2 . The judgment is said to have second order truth because 2 is the maximum of the orders of the propositional functions occurring in its analysis. Note that the propositional phrase 'all men are mortal' does not occur in this analysis as a singular term even though it may appear to so occur (when appended to 'that') in the English sentence, 'A judges that all men are mortal'. This is what Russell meant by saying that propositions are no longer to be reckoned as single entities. The phrases for the propositional functions that result from the analysis of ' $A$ judges that all men are mortal', on the other hand, all occur as singular terms in the final analysis, and it is for this reason that the propositional functions they represent must be reckoned as single entities. Indeed, without including propositional functions among the single entities combined in a judgment or belief complex, there would simply be no multiple relations theory of belief at all.

This is not to say that the multiple relations theory of belief is a viable theory after all (or at least not without serious reconstruction). Our point rather is that as far as Russell was concerned in 1910-13, propositional functions are single entities (of different r-types and orders) and can be quantified over as such, but that the same cannot be said of propositions. 
That is, propositions (in the sense of objective truths and falsehoods) are not single entities according to the Russell of 1910, and therefore they cannot be quantified over as such. This means modifying Church's characterization of $r$-types by excluding all $r$-types of the form ()$/ n$, where $n \epsilon \omega$ $-\{0\}$; or, in other words, by requiring in clause (2) of the definition of $r-$ type that $\mathrm{m} \epsilon \omega-\{0\}$ as well.

Note that rejecting propositions while retaining propositional functions in no way affects Russell's logical reconstruction of mathematics. For it is propositional functions, and not propositions, that are essential to that reconstruction. This is so because a statement about a class, i.e., a statement in which an expression for a class occurs as a singular term, is to be analyzed, according to Russell, as a statement about the extension of a propositional function; and the latter, assuming that propositional functions can be single logical subjects, is in turn to be analyzed as a statement about some (or preferably any) propositional function materially equivalent to the propositional function in question. Thus, reading ' $\hat{z}(\psi z z)$ ' as 'the class defined by $\psi$ ', Russell gives the following contextual analysis for statements in which a class appears as a single logical subject ([PM], 188):

$$
f\{\hat{z}(\psi z)\}=d f \quad(\exists \phi)((x)[\phi ! x \equiv \psi x] \& f\{\phi ! \hat{z}\}) .
$$

In this analysis, needless to say, it is essential that a propositional function can occur as a single logical subject of the analysans. In Russell's 1908 theory, on the other hand, it is propositions and not propositional functions that are reckoned as single entities; and in that regard Russell's logical reconstruction of mathematics is very much in question, since it is not $\mathrm{r}$-types of the form ()$/ \mathrm{n}$ that are then to be excluded but rather all $\mathrm{r}$ types for propositional functions that are not of this form.

Russell's pre-1910 rejection of propositional functions as single entities goes back as far as 1903, incidentally, when, as a result of his paradox, Russell was led to claim that "the $\phi$ in $\phi x$ is not a separate and distinguishable entity: it lives in the propositions of the form $\phi x$ and cannot survive analysis" ([POM], 88). Thus, since being a separable entity is the same for Russell as having the capacity of being a logical subject, there can be no propositions of the form $\psi(\phi)$, and therefore none of the form $\boldsymbol{\phi}(\phi)$ or $\sim \phi(\phi)$ as well, on this earlier view of Russell's. In other words, it was by "the recognition that the functional part of a propositional func- 
tion is not an independent entity" (ibid.) that Russell sought to avoid the contradiction that would otherwise result when his paradox was applied to propositional functions as single entities. (Cf. chapter 1, section 4, for a fuller discussion of this point.)

Despite his rejection in $[P O M]$ of propositional functions as single entities, Russell still found it "impossible to exclude variable propositional functions altogether" ([POM], 104); that is, he still admitted quantification with respect to such variables. This was because on Russell's view "wherever a variable class or variable relation [in extension] occurs, we have admitted a variable propositional function which is thus essential to assertions about every class or about every relation" (ibid.). This view was later developed by Russell into his famous "no classes" theory, first in the form of the substitutional theory of 1906 , then in the form of the 1908 theory of types, and finally in [PM]. (Cf. chapter 1, sections 6-8 for a fuller discussion of this development.) It was only in the 1910 theory of logical types, however, that Russell was finally able to give a coherent account of his "no classes" theory; for it is only in the 1910 theory that quantification over propositional functions as independently real entities is finally recognized.

\section{Propositional Functions as Properties and Relations in \\ Russell's 1910-13 Principia Mathematica-Ontology}

"Pure mathematics," Russell wrote in 1911, "is the sum of everything that we can know, whether directly or by demonstration, about certain universals" ([1911a], 293). The certain universals in question here are the independently real propositional functions that occur as "single entitics" in the analyses Russell gave in $[P M]$ of our talk of classes and numbers. "Logic and mathematics force us, then," according to this Russell of 1911, "to admit a kind of realism in the scholastic sense, that is to say, to admit that there is a world of universals and of truths which do not bear directly on such and such a particular existence. This world of universals must subsist, although it cannot exist in the same sense as that in which particular data exist" (ibid.).

Propositional functions, accordingly, are universals for Russell in his 1910-13 [PM]-ontology, and as such they may also be called properties and relations (in intension). This was already suggested by Russell in 
[1907] (p. 281), where two of his "principles" for mathematical logic are as follows:

Any propositional function of $\mathrm{x}$ is equivalent to one assigning a property to $\mathrm{x}$.

Any propositional function of $\mathrm{x}$ and $\mathrm{y}$ is equivalent to one asserting a relation between $\mathrm{x}$ and $\mathrm{y}$.

But in [1907] these two "principles" were said by Russell to be "less evident" than the others he listed there for mathematical logic; and, as already indicated, Russell attempted to do without them completely in [1908]. Nevertheless, regardless of his earlier hesitancy, and sometimes outright rejection, it is clear that Russell did assume these "principles" in his 1910-13 [PM]-ontology.

Another assumption that Russell also made in his 1910-13 ontology, albeit only implicitly, was that some properties and relations are simple while others are complex. This assumption goes back as far as [POM] where it is described as the distinction between properties and relations that are or are not logically analyzable in terms of other properties and relations. That is, if properties and relations "have been analyzed as far as possible, they must be simple terms, incapable of expressing anything except themselves" ([POM], 446); and if they are otherwise analyzable, then they must be complex. Of course, for Russell, throughout the period in which he was a logical realist, logical analysis is the same as ontological analysis; i.e., "where the mind can distinguish elements [in a logical analysis], there must be different elements to distinguish" (ibid.).

This assumption is not itself a consequence of the comprehension principle for properties and relations, incidentally; for despite the validity of the latter in $[P M]$, where propositional functions are properties and relations, nothing follows about properties and relations being themselves complex if they are specified in an instance of that principle by a complex expression for a propositional function. In other words, the complex/simple distinction is not essential to the validation of the comprehension principle (as is all too frequently assumed in the literature). Nevertheless, it is a sufficient condition if we also assume that the language of $[P M]$ is "a logically perfect language" in the sense that complex expressions for propositional functions represent (onto)logical analyses of those proposi- 
tional functions as independently real universals. This in fact was what Russell assumed in 1910-13, at least implicitly, and, as we shall see, it is not unrelated to the nominalistic validation of the comprehension principle in his later philosophy where the complexity of a propositional function is none other than its syntactical complexity as an expression. Of course properties and relations will then be distinguished from propositional functions, and in fact Russell will then in general speak of them only as simple.

The comprehension principle, incidentally, really has two forms that are valid in $[P M]$, but only one that is valid in the theory of logical types of Russell's later philosophy. These are,

$$
\text { (ヨf) }\left(\mathrm{x}_{1}\right) \ldots\left(\mathrm{x}_{\mathrm{m}}\right)\left[\mathrm{f}\left(\mathrm{x}_{1}, \ldots, \mathrm{x}_{\mathrm{m}}\right) \equiv \phi\right],
$$

and

$$
\text { (ヨf) } f=\phi\left(\hat{x}_{1}, \ldots, \hat{x}_{m}\right),
$$

where $\mathrm{f}$ is a variable of $\mathrm{r}$-type $\left(\beta_{1}, \ldots, \beta_{\mathrm{m}}\right) / \mathrm{n}$ that does not occur free in $\phi$, $\mathrm{x}_{1}, \ldots, \mathrm{x}_{\mathrm{m}}$ are variables of $\mathrm{r}$-types $\beta_{1}, \ldots, \beta_{\mathrm{m}}$, respectively, and the bound variables in $\phi$ are all of an order less than the order of $f$. Here the second form implies the first, but not conversely. The second form, given Russell's analysis of identity, is an abbreviation of

$$
(\exists \mathrm{f})(\psi)\left[\psi !\{f\} \equiv \psi !\left\{\phi\left(\hat{\mathrm{x}}_{1}, \ldots, \hat{\mathrm{x}}_{\mathrm{m}}\right)\right\}\right],
$$

which requires that propositional functions be "single logical subjects"; and this form is not even meaningful in ramified second order logic where all propositional functions are of $r$-types of the form $(i, \ldots, i) / n$, for arbitrary "level" n; i.e., where propositional functions (of arbitrary "level") have only individuals as arguments. In other words, strictly speaking, only the first form remains "significant" in Russell's later philosophy.

Nothing comparable can be said of Russell's analysis of classes, on the other hand. That is, there is no form of that analysis that remains significant in Russell's later philosophy. This is because expressions for classes are to occur as singular terms, and Russell's analysis, as described in section 1, requires that expressions for propositional functions must then also occur as singular terms; and yet it is precisely that type of occurrence that is not "significant" in ramified second order logic. 


\section{Russell's 1910-13 Commitment to Abstract Facts}

In 1910-11 Russell described his ontology as consisting simply of "an ultimate dualism" of universals and particulars. That is, "the disjunction 'universal-particular' includes all objects. We might also call it the disjunction 'abstract-concrete' " ([1910], 214). The particulars of this dualism are "particular existents" or "entities which can only be subjects or terms of relations, and cannot be predicates or relations" ([1911b], 109). (A "predicate" for Russell at this time was always a property or quality, or what he also called a concept.) A universal, on the other hand, is "anything that is a predicate or a relation" (ibid.), but which may also be a "subject" or "term" of a relation.

Particulars, incidentally, are the individuals of $[P M]$; i.e., they are the objects of r-type i. This terminology differs from that Russell used earlier in $[P O M]$ where the word 'individual' was taken as synonymous with 'term' and 'entity', or having the capacity of being a logical subject (cf. $[P O M], 43)$. This means that universals were also construed as individuals in $[P O M]$, since as logical subjects they were also "terms." In $[P M]$, on the other hand, particulars and only particulars are individuals, i.e., are of r-type $i$, which is not to say that universals have lost their individuality or capacity to occur as "terms" of other universals. The matter is really terminological, in other words, for the word used in $[P M]$ to cover the individuality of both particulars and universals is 'object'. That is, both particulars and universals are "objects" in $[P M]$, though of course they are objects of "essentially different types" ([PM], 24). As propositional functions, moreover, universals are also of different types among themselves, since some may be arguments or "terms" of others. "The division of objects into types," according to Russell, "is necessitated by the vicious circle fallacies which otherwise arise" (ibid., 161).

Now among particulars Russell included not just "existents" but "all complexes of which one or more constituents are existents, such as thisbefore-that, this-above-that, the-yellowness-of-this," etc. ([1910], 213). In 1912, Russell sometimes called these complex particulars events, and other times facts. For example, my seeing the sun and my desiring food are "events" that happen in my mind (The Problems of Philosophy, ([POP]), 49); and when "I am acquainted with my acquaintance with the sense-datum representing the sun, . . . the whole fact with which I am ac- 
quainted is 'Self-acquainted-with-sense-datum' " (ibid., 50f). Note that one of the ways that we can have knowledge of such a complex particular is "by means of acquaintance with the complex fact itself, which may (in a large sense) be called perception, though it is by no means confined to objects of the senses" (ibid., 136).

Now the importance of events or facts as complex particulars in Russell's 1910-13 ontology is that they provide the basis of his new theory of truth; that is, the theory in which truth and falsehood are no longer properties of propositions as independently real single entities, but are rather "properties of beliefs and statements" ([POP], 121). (Note that a statement for Russell is always the overt expression of a judgment or belief.) For example, in the case of a simple statement, such as that $a$ has the relation $R$ to $b$, "we may define truth ... as consisting in the fact that there is a complex corresponding to the discursive thought which is the judgment. That is, when we judge ' $a$ has the relation $R$ to $b$ ', our judgment is said to be true when there is a complex ' $a$-in-the-relation- $\boldsymbol{R}$-to- $\boldsymbol{b}$ ', and is said to be false when this is not the case" ([PM], 43).

We should note that truth and falsehood are no more univocal in Russell's new theory than they were in his earlier 1908 theory when they were properties of propositions of different orders. In particular, although beliefs or statements are themselves particular complex occurrences (and therefore are particulars), the kind of truth or falsehood each will have will depend on the highest order of the propositional functions occurring as "terms" in the belief or statement complex. For example, a statement of 'this is red' is said to have elementary truth or falsehood, while a statement of 'all men are mortal', as already explained in section 1 , will have second order truth. Similarly, a statement of 'Napoleon had all the (predicative) properties of a great general' will have third order truth or falsehood (cf. chapter 1, section 9); and of course, there can be statements or beliefs with fourth order truth or falsehood, and so on. Instead of a hierarchy of propositions as abstract entities that may be true or false, in other words, Russell's 1910-13 framework has only a hierarchy of truth and falsehood as properties of particular occurrences of beliefs and statements.

The hierarchy of truth and falsehood as properties of beliefs and statements as complex particulars fits in well with Russell's 1910-11 "ultimate dualism" of universals and particulars; i.e., with Russell's claim that 
"the disjunction 'universal-particular' includes all objects" ([1910], 214, italics added). By 1912, however, Russell came to realize that not all of the facts he needed in his "realism in the scholastic sense" could be construed as events or complex particulars. That is, with respect to Russell's 'abstract-particular' disjunction, which he had originally identified in 1910 with the 'universal-particular' disjunction, there are abstract facts as well as concrete facts (events). These are "facts about universals," and, according to Russell, "they may be known by acquaintance to many different people" ([POP], 137). For example, "the statement "two and two are four' deals exclusively with universals" (ibid., 105), and therefore the complex that makes it true is not an event or complex particular but an abstract fact. The statement itself, to be sure, as a statement made by someone at some particular time, is an event or complex particular, and as a statement about classes of classes of individuals (or rather about predicative propositional functions of predicative propositional functions of individuals) the truth it has is a property of third order (the order of the identity relation in this case). But still, since the fact that makes this statement true "deals exclusively with universals," i.e., with objects that "subsist or have being, where 'being' is opposed to 'existence' as being timeless" (ibid., 100), then the fact itself must subsist and belong to "the world of being" (ibid.).

Russell's commitment to abstract facts, it should be emphasized, cannot be brushed aside here as something that can be avoided, as though his original "ultimate dualism" of universals and particulars might suffice after all. Consider, for example, comparing Russell's ontology of universals and particulars, and now abstract facts as well, with an event ontology that is combined with the ontological commitments of one or another set theory. In the latter, there are no facts other than events (if the identification of concrete facts with events is to be retained at all), and, in particular, there are no set-theoretical facts regarding pure sets (i.e., sets whose transitive closure contain no urelements other than the empty set). Nor are any set-theoretical facts really needed, moreover, to account for the truth of statements of membership in a set. For a set, at least on the iterative concept, has its being in its members, and in that regard a set's existence (or "being," as Russell would say) is all that is needed to account for the truth or falsehood of statements ascribing membership in that set. That is, the being of a set consists in its having just the members that it 
has, and therefore no fact over and above the being of the set itself is needed to account for membership in the set. A property or relation (in intension), on the other hand, does not have its being in its instances, and for that reason its being cannot alone account for the truth of statements ascribing that property or relation to its instances. The usual gambit logical realists make here to account for such truth is to posit propositions as objective truths and falsehoods in themselves, i.e., as independently real single entities. Russell, however, had deliberately removed that option, and in consequence, he was forced to fall back on abstract facts as an additional category of his ontology beyond the events or concrete facts that make up the world of existence. In his later philosophy, when "all the propositions of mathematics and logic are assertions as to the correct use of a certain small number of words" ([1950], 306), these abstract facts are replaced by truths that are "purely linguistic." What Russell failed to see, however, was that such a replacement did not result in an equipollent system of logic.

\section{Logical Atomism and the Doctrine of Logical Types}

The status of facts as "objects" or complex single entities that can be named was important to Russell in the 1910-13 framework, and in general he went out of his way to use only perfect nominalizations of sentences (where the verb has been completely deactivated) to refer to such facts. That is why he used examples like 'my seeing the sun', 'my desire for food', ' $a$ in the relation $R$ to $b$ ', and ' $a$ having the quality $q$ ', as opposed to the imperfect nominalizations 'that I see the sun', 'that I desire food', etc. Indeed, he sometimes even used hyphens to emphasize the perfect nominalization in question, such as in 'this-before-that', 'this-abovethat', and 'the-yellowness-of-this'. Such nominalizations were necessary, according to Russell, insofar as we can be directly acquainted with the facts in question. For acquaintance is a binary relation and is to be represented by a two-place predicate expression taking only singular terms as argument expressions.

A problem does arise here regarding the logical status of facts, however. For example, insofar as the concrete fact denoted by 'this-beforethat' is a particular, it must be of the r-type $i$ of individuals. But as a complex, a fact has a logical structure, and according to Russell the com- 
plexity of that structure must somehow be represented in its logical type, which for us is its r-type. That is, as a complex a fact must have an r-type other than $i$; and therefore, assuming that no object is of more than one r-type, facts really cannot be particulars after all. This argument, or at least one with the same conclusion, was apparently forced on Russell sometime in 1913 by Ludwig Wittgenstein. Whatever his initial reluctance, and it must have been great since it meant giving up completing the manuscript of his 1913 Theory of Knowledge, Russell came to accept the conclusion by the end of 1913.

Now it is significant that in accepting this conclusion Russell went on to claim that "only particulars can be named" ("The Philosophy of Logical Atomism," ([PLA]), 267), and therefore that facts cannot be named at all. For example, in January 1914, Russell wrote that although "an observed fact ... does not differ greatly from a simple sense-datum as regards its function in giving knowledge," nevertheless "its logical structure is very different ... from that of sense: sense gives acquaintance with particulars, and is thus a two-term relation in which the object can be named but not asserted,... whereas the observation of a complex fact, which may be suitably called perception, is not a two-termed relation, but involves the propositional form on the object-side, and gives knowledge of a truth, not mere acquaintance with a particular" ([1914], 147). In other words, it was no longer even meaningful, no less true, for Russell that "the complex ' $a$-in-the-relation- $R$-to- $b$ ' may be capable of being perceived... as one object" ([PM], 43). That is, this perception was no longer "a relation of two terms, namely, ' $a$-in-the-relation- $R$-to- $b$ ', and the percipient" (ibid.). And the reason, Russell claims, is that the logical structure of a fact precludes it from being the sort of entity that can be named, i.e., the sort of entity that can stand as a "term" in a relation. "You cannot name a fact.... You can never put the sort of thing that makes a proposition to be true or false in the position of a logical subject" ([PLA], 188).

Before taking up this rather extraordinary claim, let us note that as so far defined no r-type is the r-type of a fact, and therefore in a trivial sense no fact can be a "logical subject" according to the theory of r-types. The reason why this is so is that every r-type other than $i$ is the r-type of a propositional function, and facts are not propositional functions. Resurrecting propositional $r$-types of the form ()$/ \mathrm{n}$ for facts will not do, more- 
over, since these fail to indicate both the number and the r-types of the constituents of a fact.

We can rectify this situation, however, if we assume along with the Russell of the 1910-13 ontology that every fact consists of some one relation actually relating the remaining constituents of that fact. (This assumption of Russell's goes as far back as the Principles; cf. [POM], 52.) For example, the relation that Russell calls "formal implication" (and represented by $(\mathrm{x})[\hat{\phi} ! \mathrm{x} \supset \hat{\psi} ! \mathrm{x}]$ in section 1 ) is the relating relation of a general fact (cf. $[P M], 138)$, and "the asymmetrical relation of predication" is the relating relation of a fact corresponding to a true subject-predicate sentence (cf. [1911b]). Following Russell's later usage (adopted from Wittgenstein's Tractatus), we shall call the relating relation of a fact the component relation of that fact, while the "terms" or "logical subjects" of that relation will be called simply the constituents of the fact. We can now revise the definition of r-type given in section 1 by supplementing that definition with the following clause:

$$
\begin{aligned}
& \text { If } \mathrm{m}, \mathrm{n} \in \omega-\{0\}, \mathrm{m} \geqslant 2, \text { and } \beta_{1}, \ldots, \beta_{\mathrm{m}} \text { are given } \mathrm{r} \text {-types, } \\
& \text { then there is an r-type }\left[\left(\beta_{1}, \ldots, \beta_{\mathrm{m}}\right) / \mathrm{n}\right] \text { to which belong all } \\
& \text { and only facts whose component relation is of } \mathrm{r} \text {-type } \\
& \left(\beta_{1}, \ldots, \beta_{\mathrm{m}}\right) / \mathrm{n} \text { and whose constituents are of } \mathrm{r} \text {-type } \beta_{1}, \ldots \\
& \beta_{\mathrm{m}} \text { respectively; and the order of such a fact is the order of } \\
& \text { its component relation. }
\end{aligned}
$$

Of course it now follows that no concrete fact is a particular, since particulars are all of r-type i and no fact is of r-type i. But that-as far as the theory of logical types as the theory of r-types is concerned-has nothing to do with Russell's new or post- $[P M]$ claim that you cannot name a fact, or that a fact cannot be a logical subject.

There is no reason, for example, at least as far as the theory of r-types is concerned, why there cannot be different types of relations of acquaintance, just as there are on Russell's multiple relations theory different types of relations of belief. Only one of these acquaintance relations will in fact be a relation between individuals; others will be relations between individuals and facts (of a given r-type) or between individuals and universals (of a given r-type). (Russell also called acquaintance with universals conceiving; cf. [1910], 212.) Also, as far as the theory of r-types is concerned, there is no reason why all and only individuals should be particu- 
lars, i.e., why particulars should constitute a distinct logical category. After all, if concrete facts can be logical subjects, then why shouldn't they be called complex particulars, just as Russell in fact called them in his 191013 ontology?

It is not just facts that Russell now says cannot be named, it should be noted, but anything that suggests "the form of a proposition" ([PLA], 205). A property or quality, for example, cannot be named by a nominalized predicate, since "a predicate," according to Russell's new or post$[P M]$ view, "can never occur except as a predicate. When it seems to occur as a subject [i.e., as a nominalized predicate], the phrase wants amplifying and explaining, unless, of course, you are talking about the word itself" (ibid.). Similarly, "a relation can never occur except as a relation, never as a subject" (ibid., 206). "All propositions in which an attribute or a relation seems to be the subject," in other words, "are only significant if they can be brought into a form in which the attribute is attributed or relation relates. If this were not the case, there would be significant propositions in which an attribute or a relation would occupy a position appropriate to a substance, which would be contrary to the doctrine of types, and would produce contradictions" ("Logical Atomism" ([LA]), 337f).

Note that here we have another extraordinary claim: facts and universals cannot be logical subjects, and therefore cannot be named, because that would be contrary to the doctrine of types, and would produce contradictions. What is so extraordinary about this is that facts and universals are logical subjects in the theory of r-types, and as a theoretical account of the doctrine of logical types, the theory of r-types was designed explicitly so as not to produce contradictions. Apparently, Russell has somehow replaced his earlier version of the doctrine of logical types with a new or much restricted version; and it is not at all clear how well aware he was of the consequences of this move.

Note also that on this new version of the doctrine of types, Russell must reject his multiple relations theory of belief, as well of course as the view that we can be acquainted with facts and universals. Thus, besides "the impossibility of treating the proposition believed as an independent entity, entering as a unit into the occurrence of the belief" ([PLA], 226.), there is now also "the impossibility of putting the subordinate verb on a level with its terms as an object term in the belief" (ibid.). "That is a point," Russell observed, "in which I think that the theory of judgment 
which I set forth once in print some years ago was a little unduly simple, because I did then treat the object verb as if one could put it as just an object like the terms, as if one could put 'loves' on a level with Desdemona and Cassio as a term for the relation 'believe' "(ibid.) in the case of Othello believing that Desdemona loves Cassio. (Note that Russell uses 'verb' to stand for both the word and the attribute or relation the word stands for; cf. [PLA], 217.)

Now it is clear, as these observations indicate, that Russell has changed or seriously modified his 1910-13 ontology, and that somehow the change involves a new version of the doctrine of types. Thus, in 1924 Russell writes that "the doctrine of types leads to ... a more complete and radical atomism than any that I conceived to be possible twenty years ago" ([LA], 333), which in this case includes the 1910-13 ontology. This complete and radical atomism is of course Russell's form of logical atomism, the justification of which he claims is none other than "the justification of analysis" ([PLA], 270). On this view, "you can get down in theory, if not in practice, to ultimate simples, out of which the world is built, and ... those simples have a kind of reality not belonging to anything else" (ibid.); i.e., each simple has a kind of reality or mode of being that is unique to the entities of that kind (and which is the same as its logical type). "Simples ... are of an infinite number of sorts. There are particulars and qualities and relations of various orders, a whole hierarchy of different sorts of simples" (ibid.). Aside from simples, "the only other sort of object you come across in the world" are facts (ibid.). That is, in the ontology of logical atomism, there are only simples and facts. Everything else is what Russell called a "logical fiction" (cf. [PLA], 253f).

The hierarchy of different sorts of simples that is in question here, it should be noted, is not the hierarchy of r-types (where properties and relations can be logical subjects). For by the "order" of a relation Russell means in this context only the degree or adicity of that relation (cf. [PLA], 206f). That is, he does not mean "order" in the sense defined in section 1. In that regard, the r-types of the hierarchy of simples, i.e., of particulars, qualities and relations of various "orders" now intended by Russell, can be indicated as follows, namely: $i,(i) / 1,(i, i) / 1,(i, i, i) / 1$, and so on ad infinitum. (Note that as simples, no quality or relation has a "level" higher than 1 ; i.e., each is "predicative" in the sense of section 1). It is only first order properties and relations, in other words, and even then only simple 
first order properties and relations, that are involved in the ontology of logical atomism. Of course, quantifiers that "refer" to these simple properties and relations are no less significant than quantifiers that refer to particulars as individuals, which means that some restricted form of second order logic is needed for the representation of this ontology. Indeed, the sentences of this restricted form of second order logic are precisely what Russell later called the atomistic hierarchy of sentences; that is, the hierarchy of sentences obtained from atomic sentences by the three operations of substitution, combination and generalization (cf. [Inq], 187).

Note that by the operation or principle of substitution Russell only means that an atomic sentence $R_{\mathrm{n}}\left(a_{1}, \ldots, a_{\mathrm{n}}\right)$ "remains significant if any or all of the names are replaced by any other names, and $R_{\mathrm{n}}$ is replaced by any other n-adic relation" (ibid., 185). Truth-functional or molecular compounds of atomic sentences are then obtained by iterated application of the stroke-operation of combination (having the truth-table of 'either not ... or not ...'); and "given any sentence containing either a name ' $a$ ' or a word ' $R$ ' denoting a relation or predicate, we can construct a new sentence in two ways" (ibid.), according to Russell, by the operation of generalization; that is, quantification is significant with respect to both the subject and relation or predicate positions of atomic sentences. The resulting "hierarchy" of sentences, needless to say, consists only of second order sentences; that is, it consists of sentences that are significant in second order logic where there are no higher order universals of an $\mathrm{r}$-type $\left(\beta_{1}, \ldots\right.$ ,$\left.\beta_{\mathrm{m}}\right) / \mathrm{n}$, where $\beta_{\mathrm{i}}$, for some $\mathrm{i}$, is the r-type of a property or relation. This means that expressions for the higher order universals that Russell took numbers to be in his 1910-13 ontology are no longer significant in Russell's new theory of types. And yet, according to Russell's version of the principle of atomicity, "everything we wish to say can be said in sentences belonging to the 'atomistic hierarchy' " ([Inq], 160).

\section{Propositional Functions as Linguistic Conveniences}

In considering whether the atomistic hierarchy of sentences "can constitute an 'adequate' language, i.e., one into which any statement in any language can be translated" ([Inq], 187), Russell asks if we can "be content with names, predicates, dyadic relations, etc., as our only variables, or do we need variables of other kinds?" (ibid.). This question, we are told, "is 
concerned with generalization and is relevant in solving the paradoxes" (ibid.). The other kinds of variables Russell has in mind here are propositional variables and propositional function variables (or what Russell also called variables propositions and variable functions).

Now by a proposition in his post- $[P M]$ view Russell means not an objective truth or falsehood, but "a sentence in the indicative" ([PLA], 185), i.e., "a sentence asserting something" (ibid.). In other words, "a proposition is just a symbol", and in particular "it is a complex symbol in the sense that it has parts which are also symbols" (ibid.). (Russell sometimes also means by a proposition an image-proposition (cf. [1919] and The Analysis of Mind, ([AOM]); but we shall ignore image-propositions here since they correspond only to atomic sentences and do not contain propositional functions.) A propositional function, similarly, "is simply any expression containing an undetermined constituent, or several undetermined constituents, and becoming a proposition as soon as the undetermined constituents are determined" (ibid., 230). Of course, as an expression that can be mentioned and talked about as such, a propositional function is a "single entity." But being mentioned is not the same as being used, and as for its use in logical syntax "the only thing really that you can do with a propositional function is to assert either that it is always true, or that it is sometimes true, or that it is never true" (ibid.); that is, otherwise than being referred to as an expression, "a propositional function is nothing" (ibid.). This means that as an expression that is being used rather than mentioned, a propositional function cannot occur as the grammatical subject of a proposition. This is why Russell in his 1925 introduction to the second edition of [PM] claims that "there is no logical matrix of the form $\mathrm{f} !(\phi ! \hat{z})$. The only matrices in which $\phi ! \hat{z}$ is the only argument are those containing $\phi ! a, \phi ! b$, $\phi ! c, \ldots$, where a, b, c are constants" ([1925], xxxi), and of course these are matrices in which $\phi ! z \hat{z}$ does not occur as a singular term or logicogrammatical subject. Indeed, this is precisely what Russell means by his new fundamental assumption that "a function can only appear in a matrix through its values" (ibid., xxix).

Note that by a matrix Russell means in this context any propositional function (expression) that "has elementary propositions as it values" (ibid., xxii), where an elementary proposition is either an atomic proposition or a truth-functional compound of atomic propositions (ibid., xvii). 
Note also that an $\mathrm{n}$-adic relation symbol $\mathrm{R}_{\mathrm{n}}$ "cannot occur in a atomic proposition $R_{m}\left(x_{1}, \ldots, x_{m}\right)$ unless $n=m$, and then can only occur as $R_{m}$ occurs, not as $\mathrm{x}_{1}, \mathrm{x}_{2}, \ldots, \mathrm{x}_{\mathrm{m}}$ occur" (ibid.); i.e. relation symbols are not allowed to occur as singular terms (the way they were allowed to occur, e.g., in Russell's 1910-13 multiple relations theory of belief or in what he then called higher order matrices). Finally, note that what ' $\phi$ ! $a$ ' stands for, according to Russell, is any elementary proposition that contains an atomic sentence of the form $R_{n}\left(a, b_{1}, \ldots, b_{n-1}\right)$. That is, $\phi ! a$ is an elementary proposition in which, strictly speaking, $\phi ! z$ does not occur as an "argument" at all once we are given the predicate and relation symbols upon which any application of Russell's logical syntax is to be based. This is why Russell says in his 1925 introduction to $[\mathrm{PM}]$ that the "peculiarity of functions of second and higher order is arbitrary" (p. xxxii), and that in fact by adopting predicate and relation variables (i.e., predicate variables of different adicities) we can avoid the notation for propositional functions altogether (ibid.). In other words, no new variables are really needed, according to Russell, beyond those already occurring in the atomistic hierarchy of sentences.

Now because "the logic of propositions, and still more of general propositions concerning a given argument, would be intolerably complicated if we abstained from the use of variables functions" (ibid.), Russell does go on to include propositional variables and function variables into his new logical syntax. But, despite appearances to the contrary, these new variables all belong to ramified second order logic; that is, they are not allowed to occur as singular terms or logicogrammatical subjects of the new sentences formed by their addition to the atomistic hierarchy. Russell's notation can be deceptive in this regard, however; for even though "there is no logical matrix of the form $\mathrm{f} !(\phi ! \hat{z})$ " (p. xxxi), i.e., a matrix where $f$ is a second order variable of $r$-type $(i / 1) / 1$, nevertheless, according to Russell, there are logical matrices of the form $f !\left(\phi ! \hat{z}, x_{1}, x_{2}, \ldots\right.$ , $\mathrm{x}_{\mathrm{n}}$ ), where "we call $\mathrm{f}$ a 'second-order function' because it takes functions among its arguments" (ibid.). A matrix of this form, however, "is always derived from a stroke-function

$$
\mathrm{F}\left(\mathrm{p}_{1}, \mathrm{p}_{2}, \mathrm{p}_{3}, \ldots, \mathrm{p}_{\mathrm{n}}\right)
$$

by substituting $\phi ! \mathrm{x}_{1}, \phi ! \mathrm{x}_{2}, \ldots, \phi ! \mathrm{x}_{\mathrm{n}}$ for $\mathrm{p}_{1}, \mathrm{p}_{2}, \ldots, \mathrm{p}_{\mathrm{n}}$. This is the sole method for constructing such matrices" (ibid., italics added). Note that 
the propositional variables $\mathrm{p}_{1}, \ldots, \mathrm{p}_{\mathrm{n}}$ do not occur in a stroke-function as singular terms, but as "arguments" of a sentential connective (viz., the stroke connective having the truth-table of 'either not ... or not ...'). This means that the substitution of $\phi ! \mathrm{x}_{1}, \phi ! \mathrm{x}_{2}, \ldots, \phi ! \mathrm{x}_{\mathrm{n}}$ for $\mathrm{p}_{1}, \mathrm{p}_{2}, \ldots, \mathrm{p}_{\mathrm{n}}$ in a stroke-function does not result in a proposition in which $\phi ! \hat{z}$ occurs as a singular term, despite appearances to the contrary in Russell's way of representing this substitution as $\mathrm{f} !\left(\phi ! \hat{z}, \mathrm{x}_{1}, \mathrm{x}_{2}, \ldots, \mathrm{x}_{\mathrm{n}}\right)$. In other words, despite appearances, $f$ is not occurring in this matrix as an $(n+1)$-ary second order variable of $\mathrm{r}$-type $((\mathrm{i}) / 1, \mathrm{i}, \ldots, \mathrm{i}) / 1$, but as an $\mathrm{n}$-ary second order variable of $\mathrm{r}$-type $(\mathrm{i}, \ldots, \mathrm{i}) / 2$. This is why Russell says that "since $\phi$ can only appear through its values, it must appear, in a logical matrix, with one or more variables arguments" (ibid., italics added). ${ }^{3}$

Now in regard to generalization and the ramification of propositional functions, note that according to Russell "when we have a general proposition $(\phi) . \mathrm{F}\{\phi ! \hat{z}, \mathrm{x}, \mathrm{y}, \ldots\}$, the only values $\phi$ can take are matrices, so that functions containing apparent variables are not included" (ibid., xxxiii). However, "we can, if we like, introduce a new variable to denote not only functions such as $\phi ! \hat{x}$, but also such as

$$
\text { (y). } \phi !(\hat{x}, y),(y, z) \cdot \phi !(\hat{x}, y, z), \ldots(\exists y) \cdot \phi !(\hat{x}, y), \ldots ;
$$

in a word, all such functions of one variable as can be derived by generalization from matrices containing only individual variables" (ibid.). For this purpose, Russell introduces the variables $\phi_{1}, \psi_{1}, \chi_{1}$, etc.; i.e., "the suffix 1 is intended to indicate that the values of the functions may be firstorder propositions, resulting from generalization in respect of individuals" (ibid.). "Theoretically," according to Russell, "it is unnecessary to introduce such variables as $\phi_{1}$, because they can be replaced by an infinite conjunction or disjunction" (ibid.).

Of course, "when the apparent variable is of higher order than the argument, a new situation arises. The simplest cases are

$$
(\phi) . f !(\phi ! \hat{z}, x),(\exists \phi) . f !(\phi ! z, x) .
$$

These are functions of $\mathrm{x}$ [where $\mathrm{f}$ is of $\mathrm{r}$-type (i)/2, and not of $\mathrm{r}$-type ((i)/ $1, \mathrm{i}) / 1$ as might appear from Russell's notation], but are obviously not included among the values for $\phi ! x$ (where $\phi$ is the argument)" (ibid., xxxiv). Russell's original reason for this restriction of the values of $\phi ! x$ was that paradoxes would otherwise ensue, including in particular his 
own paradox of predication. But that reason assumes that $\mathrm{f}$ is of $\mathrm{r}$-type $((\mathrm{i}) / 1, \mathrm{i}) / 1$ in the above examples, and not of $\mathrm{r}$-type (i)/2, as is required in Russell's new "atomistic" theory. That is, given Russell's fundamental new assumption that "a function can only appear in a matrix through its values," Russell's own paradox is not even formulable, since it depends on propositional functions being expressions that can occur as singular terms of second order matrices (or, as in Frege's Grundgesetze, on propositional functions having certain abstracts as their singular term counterparts). In other words, no paradox would be forthcoming in Russell's new or restricted logical syntax even if we were to allow the "values" of $\phi ! x$ to include propositional functions in which $\phi$ occurs as a bound variable. (This of course is just the situation that obtains in standard impredicative second order logic.)

There is a reason, nevertheless, why the "values" of $\phi ! x$ should not include propositional functions in which $\phi$ or another function variable has a bound occurrence, and that is Russell's new or post- $[P M]$ nominalistic construal of propositional functions. For "in the language of the second order, variables denote symbols, not what is symbolized" ([Inq], 192), and in that regard, of course, they cannot themselves be among the symbols they "denote." That is, bound propositional function variables are to be given a substitutional and not an "objectual" interpretation (as they were in Russell's original 1910-13 theory). On this interpretation, to attempt to make the "values" of $\phi ! x$ include propositional functions that contain bound occurrences of $\phi$ "is like attempting to catch one's own shadow. It is impossible to obtain one variable which embraces among its values all possible functions of individuals" ([1925], xxxiv).

Of course, "we can adopt a new variable $\phi_{2}$ which is to include functions in which $\phi !$ ż can be an apparent variable" (ibid.); but then "we shall obtain new functions" (ibid.), and in this way go on to adopt new variables $\phi_{3}, \phi_{4}$, etc. Each of the new variables will belong to a language one order higher than the language whose propositional functions are the substituends or "values" of those variables, and therefore of course none of the substituends or "values" of these new variables can contain bound occurrences of those variables themselves. But all of these variables, it should be emphasized, will be variables of ramified second order logic; i.e., they will have as substituends only propositional functions of individuals, albeit functions of higher and higher "levels," and in that sense 
of higher and higher "orders" as well. For, as defined in section 1, the order of an m-ary propositional function of $r$-type $(i, \ldots, i) / n$ will be the same as its level; and of course that is why the languages generated by the addition of the new variables will be one order higher than the language whose propositional functions are the substituends or "values" of those variables. This means that the higher order languages of Russell's later philosophy are not the higher order languages of the simple theory of types, and indeed this is why according to Russell, "my hierarchy of languages is not identical with Carnap's or Tarski's" ([Inq], 60). For on Russell's "atomistic" view, "what is necessary for significance is that every complete asserted proposition should be derived from a matrix by generalization, and that, in the matrix, the substitution of constant values for the variables should always result, ultimately, in a stroke-function of atomic propositions. We say 'ultimately', because, when such variables as $\phi_{2} \hat{z}$ are admitted, the substitution of a value for $\phi_{2}$ may yield a proposition still containing apparent variables, and in this proposition the apparent variables must be replaced by constants before we arrive at a stroke function of atomic propositions. We may introduce variables requiring several such stages, but the end must always be the same: a stroke-function of atomic propositions" (ibid., xxxv, italics added). In other words, ultimately, according to Russell, "there is ... no reason to admit as fundamental any variables except name-variables and relation-variables (in intension)" ([Inq], 192), where the latter cannot themselves occur as singular terms. That is, in the end, according to Russell, a proposition is significant only if it can be translated into the atomistic hierarchy of sentences.

It is in this sense, accordingly, that propositional functions are merely linguistic conveniences in Russell's later philosophy. And, indeed, as a claim about the reducibility of the truth-conditions of ramified second order logic to the truth-conditions of the atomistic hierarchy of sentences, such a view is completely unproblematic. Where Russell errs in his later philosophy is in thinking that everything that could be said in his original theory of types can also be said in the atomistic hierarchy, or what comes to the same thing, that his earlier theory of types is equipollent to ramified second order logic. In particular, Russell's own analysis of classes in terms of propositional functions is no longer available to him in his later philosophy; and apparently the reason he failed to see this was his new 
way of representing a logical matrix. Russell's claim, accordingly, that "truth in pure mathematics is syntactical" ([MPD], 220) and that "numbers are classes of classes, and classes [as propositional functions] are symbolic conveniences" (ibid., 102), cannot be justified, since in order to talk of numbers as classes of classes, we must first be able to use expressions for classes as singular terms, which in Russell's framework ultimately means that we must be able to use propositional functions as abstract singular terms, and not merely as expressions that can be asserted as being always true, or sometimes true, or never true.

\section{Russell's Weakened Form of the Principle of Atomicity}

As originally formulated by Wittgenstein, the principle of atomicity is the thesis that "every statement about complexes can be analyzed into a statement about their constituent parts, and into those propositions which completely describe the complex" (Tractatus, 2.0201). For Russell, the technical form of this principle became, as we have said, the thesis that every significant sentence can be translated into the atomistic hierarchy of the sentences of an ideal or logically perfect language (whose logical syntax turns out to be that of ramified second order logic). But since, according to Russell, all complexes are facts and facts cannot be named, it follows that the names of such a language can only denote simple particulars.

This makes the practical application of such a language very difficult, if not impossible, it should be noted, since Russell himself always maintained that what we take to be a simple particular may in the end really be complex and susceptible to further analysis. Indeed, by 1940, Russell came to the conclusion that "everything that there is in the world I call a 'fact'. The sun is a fact; Caesar's crossing the Rubicon was a fact; if I have a toothache, my toothache is a fact" (Human Knowledge: Its Scope and Limits ([HK]), 43). Facts in this sense, it should be noted, "are to be conceived as occurrences" ([Inq], 268), i.e., as events.

Now events, from 1914 to 1940, were the original simple particulars of Russell's atomist ontology, with ordinary physical objects being somehow analyzed as complexes consisting of a "compresence" of events (cf. [LA], 341). That analysis is very much in doubt, however, insofar as complexes cannot be named and ascribed properties and relations in Russell's atomistic hierarchy. That is, just as Russell's earlier analysis of classes 
and numbers is no longer significant in his new logical syntax, so too his analysis of physical objects as a series of events is at least problematic, if not also nonsignificant in its allowing such complexes to have properties and stand in various relationships to one another. In addition, most, if not all, events will have an internal complexity of their own, and so they will not really be the simple particulars of an atomist ontology after all.

Russell never doubted the adequacy (or availability within the atomistic hierarchy) of his analysis of physical objects as complexes of events, it must be said; but he did agree, at least from 1940 on, that most events, notwithstanding their status as particulars, were themselves complexes. Their constituents, it turned out, or at least so Russell proposed, were simple qualities. Thus, from 1940 on, events were no longer the simple particulars of Russell's atomist ontology, but were reconstrued as complexes of simple qualities. For Russell, this meant that words for qualities, such as 'red', 'blue', 'hard', 'soft', etc., are "names in the syntactical sense" ([Inq], 89) of his ideal language. For example, according to Russell, "wherever there is, for common sense, a 'thing' having the quality $\mathrm{C}$, we should say, instead, that $\mathrm{C}$ itself exists in that place, and that the 'thing' is to be rcplaced by the collection of qualities existing in the place in question. Thus ' $C$ ' becomes a name, not a predicate" (ibid., 93). This does not mean that properties and relations in general can now be named; for Russell continued to insist right until the end that "relation-words ought only to be employed as actually relating and that sentences in which such words appear as subjects are only significant when they can be translated into sentences in which the relation-words perform their proper function of denoting a relation between terms. Or as it may be put in other words: verbs are necessary, but verbal nouns are not" ([MPD], 173).

Now what is important about this modification in Russell's ontology is that simple qualities are not the only particulars there are. That is, in Russell's ideal language of the atomistic hierarchy, at least from 1940 on, names may denote not only simple qualities but complexes of such as well (cf. $[H K], 84$ ). This in fact is what Russell means by the weakened form of the principle of atomicity; that is, the form in which the principle "is not to be applied to everything that is in fact complex, but only to things named by complex names" ([Inq], 251). "A name $\mathrm{N}$ may be in fact the name of a complex, but may not itself have any logical complexity, i.e., any parts that are symbols. This is the case with all names that actually oc- 
cur. Caesar was complex, but 'Ceasar' is logically simple, i.e., none of its parts are symbols" ([Inq], 251). On the other hand, "though 'Caesar' is simple, 'the death of Caesar' is complex" (ibid.), and according to the principle of atomicity, it is to be analyzed into a statement about its constituent parts. In other words, although facts in the sense of events can be named in Russell's later philosophy after all, complex names of facts must still be analyzed and are not allowed to occur as names in the logical language based on the atomistic hierarchy of sentences (cf. [Inq], 309).

This weakening of the principle of atomicity does allow Russell to translate sentences about physical objects into the logical language of his atomistic hierarchy, even though physical objects are ultimately themselves complexes of events, which in turn are complexes of compresent simple qualities. The translation, however, must never be such as to syntactically represent physical objects both as particulars and as complexes, since statements about complexes as single entities or "logical subjects" will have no counterparts in the atomistic hierarchy. How satisfactory a resolution of the problem of the practical application of Russell's ideal language this comes to in the end, we shall not attempt to assess here. For it still remains true in any case that Russell's original analysis of classes and numbers in terms of propositional functions as single entities will have no counterpart in his atomistic hierarchy of sentences.

\section{NOTES}

1 Cf. [CRR]. We take $\omega$ to be the set of natural numbers; thus, ' $m \epsilon \omega$ ' is read ' $m$ is a natural number', and ' $\mathrm{\epsilon} \omega-\{0\}$ ' is read ' $n$ is a natural number other than 0 '. We assume, incidentally, that the definition applies to expressions as well as to what the expressions stand for.

2 This notion of "level" should not be confused with Frege's. It corresponds, though not exactly, to Russell's notion of "order" in $[P M]$. We have retained Church's terminology here, since we are after all using his characterization of the r-types. We should note, however, that we use the phrase 'ramified second order logic' with its now standard meaning (as described, e.g., in [IML], section 58); i.e., as referring to the system all of the propositional functions of which have r-types of the form $(i, \ldots, i) / n$, for arbitrary "level" $n$. These functions have only individuals as arguments, and therefore, as defined above, their "order" is the same as their "level". This means that functions of every "order" are among the functions of ramified second order logic, even though they always have only individuals as their arguments. I believe, incidentally, that a confusion of the different notions of order and level in part explains why Russell failed to 
see that the theory of types in his later philosophy was not the same as the theory he described in $[P M]$.

3 We should note, incidentally, that the use of the exclamation mark following the variable $f$ no longer means that $f$ is "predicative" in the sense defined in section 1 . Rather, in Russell's 1925 introduction to [PM], it simply means that the function has elementary propositions as its values (cf. p. xxviii).

\section{References}

[IML] Church, Alonzo, Introduction to Mathematical Logic, vol. 1 (Princeton, 1956).

[CRR] Church, Alonzo, "Comparison of Russell's Resolution of the Semantical Antinomies with that of Tarski," Journal of Symbolic Logic, 41 (1976): 747-60.

[POM] Russell, Bertrand, The Principles of Mathematics, $2 \mathrm{~d}$ ed. (NY., Norton \& Co., 1938).

[1907] Russell, Bertrand, "The Regressive Method of Discovering the Premises of Mathematics," in [Essays].

[1908] Russell, Bertrand, "Mathematical Logic as Based on the Theory of Types," American Journal of Mathematics 30 (1908):222-62; reprinted in [LむK].

[1910] Russell, Bertrand, "Knowledge by Acquaintance and Knowledge by Description," Proceedings of the Aristotelian Society for 1910-11; reprinted in $[M \nLeftarrow L]$, to the pagination of which we refer.

$[P M]$ Russell, Bertrand and Alfred Whitehead, Principia Mathematica, vol. 1 (1910), vol. 2 (1912), and vol. 3 (1913) (London: Cambridge Univ. Press,).

[1911a] Russell, Bertrand, "The Philosophical Implications of Mathematical Logic," Revue de Metaphysique et de Morale 19 (1911):281-91. Reprinted in [Essays], to the pagination of which we refer.

[1911b] Russell, Bertrand, "On the Relations of Universals and Particulars," Proceedings of the Aristotelian Society for 1911-12; reprinted in [LむK], to the pagination of which we refer.

[POP] Russell, Bertrand, The Problems of Philosophy (Oxford Univ. Press, 1912); reset and reprinted in 1957.

[1914] Russell, Bertrand, “The Relation of Sense-Data to Physics," Scientia (1914); reprinted in $[M \& L]$.

[MঊL] Russell, Bertrand, Mysticism and Logic (London: George Allen \& Unwin, 1917).

[PLA] Russell, Bertrand, "The Philosophy of Logical Atomism," Monist 28 (1918): 495-527; reprinted in $[L \nLeftarrow K]$, to the pagination of which we refer.

[1919] Russell, Bertrand, "On Propositions: What They Are and How They Mean," Proc. of the Aristotelian Society, supp. vol. 2 (1919): 1-43; reprinted in [LむK].

$[A O M]$ Russell, Bertrand, The Analysis of Mind (London: George Allen \& Unwin, 1921).

[LA] Russell, Bertrand, "Logical At七mism," in Contemporary British Philosophy, J.H. Muirhead, ed. (1924); reprinted in [LむK], to the pagination of which we refer. 
[1925] Russell, Bertrand, "Introduction to the Second Edition," in second edition of [PM], (1925):xiii-xlvi.

[Inq] Russell, Bertrand, An Inquiry into Meaning and Truth (London: George Allen \& Unwin, 1940); reprinted by Penguin Books, Baltimore (1962), to the pagination of which we refer.

[HK] Russell, Bertrand, Human Knowledge: Its Scope and Limits (N.Y.: Simon \& Schuster, Inc., 1948).

[1950] Russell, Bertrand, "Is Mathematics Purely Linguistic?" in [Essays].

[MPD] Russell, Bertrand, My Philosophical Development (London: George Allen \& Unwin, 1959).

[LむK] Russell, Bertrand, Logic and Knowledge, edited by R.C. Marsh (London: George Allen \& Unwin, 1956).

[Essays] Russell, Bertrand, Essays in Analysis, edited by D. Lackey (N.Y.: George Braziller, 1973). 


\section{Logical Atomism and Modal Logic}

Logical atomism has been construed as both a realist and a nominalist ontology. Despite their different ontological commitments, proponents of both types of atomism have tended to agree that modal operators for necessity and possibility, and thereby strict entailment too, are totally alien to the ontological grammar of logical atomism. The reason for this, apparently, is that any inclusion of modal operators in the ontological grammar of logical atomism, whether that grammar be of the nominalist or realist variants, would represent a commitment to internal properties and relations with material content. And in logical atomism, of course, all internal properties and relations, be they of objects or of situations, are formal and not material in nature. (Cf. Wittgenstein, Tractatus Logico-Philosophicus, ([TR]), 4.122).

However, to the contrary, we shall argue that not only are propositional connectives for logical necessity and possibility, and thereby strict entailment too, no more alien to the ontological grammar of logical atomism than are connectives for conjunction and disjunction, but, moreover, that the formal or internal properties and relations of objects and situations in the ontology cannot be adequately represented by the propositional forms of that grammar unless connectives for logical necessity and possibility are included (or definable by others so included) therein. 
That is, we shall argue that connectives for logical necessity and possibility, together with their proper "logico-syntactical employment" ([TR], 3.327), represent formal, and not material, internal "properties," and, moreover, that these formal, internal "properties" are part of the ontology of logical atomism and cannot be adequately represented without the inclusion of such connectives in the ontological grammar of any formal system purporting to represent that ontology. ${ }^{1}$

Our position and argument, incidentally, applies only to modal operators for logical necessity and possibility. All other modal operators, we agree, because they purport to represent internal "properties" or "relations" with real material content (e.g., causality, and even temporality via tense logic), are strictly prohibited within the metaphysical framework of logical atomism. "Superstition is nothing but belief in the causal nexus" ([TR], 5.1361). "The only necessity that exists is logical necessity" ([TR], 6.37).

Moreover, our concern here shall be with logical atomism as the metaphysical framework for a type of formal ontology. Our concern will not be with logical atomism as the framework for either a theory of meaning or a theory of knowledge. Accordingly, neither the Carnapian theory of Protokolsätze nor the Tractarian picture theory of meaning are essential to our present purely ontological considerations. We should note, however, that the Tractarian theory of elementary propositions as pictures contains both a theory of predication and a theory of meaning. It is the theory of predication that is an essential part of the ontology of logical atomism.

In the present chapter we shall limit our formal developments to the level of analysis dealing solely with propositional connectives. Our next chapter will deal with nominalist logical atomism where only individual variables are bindable but where atomism's theory of predication enters the ontological grammar in a fundamental way. That chapter will also contain a description of several variants of realist logical atomism, one in which material properties and relations of objects are themselves objects, and another where material properties and relations of objects, though indicated by bound predicate variables (as in the first variant of realism), are not themselves objects (values of individual variables) but are nexuses or modes of configurations of objects (as they are in nominalism where they are not indicated by bound predicate variables). 


\section{Atomic Situations and Complementation}

The variant of logical atomism we shall consider takes an un-Tractarian view of negation and nonexistence in regard to atomic situations or states of affairs. According to this variant, there exists corresponding to each atomic situation (Sachlage) another atomic situation that is its complement. Complementation, moreover, is symmetrical: the complement of the complement of an atomic situation is just that situation itself. In addition, between complementary atomic situations, one exists (is-the-case) while the other "non-exists" (is-not-the-case). In this regard, the (mode of) being of an atomic situation is not its existence, i.e., its facticity, if indeed it does exist, but rather its being-the-case-or-not-the-case. Accordingly, even though only one of a pair of complementary atomic situations exists (is-the-case), the other nevertheless has being qua atomic situation in its being-not-the-case.

This distinction between being and existence apparently qualifies the Tractarian view that atomic situations are independent of one another. It is not true here that from the existence or nonexistence of one atomic situation it is impossible to infer the existence or nonexistence of another (cf. 2.062). It is true here, however, that with respect to noncomplementary atomic situations it is impossible to infer the existence or nonexistence of one from the existence or nonexistence of the other. It is this qualified form of the Tractarian thesis that is fundamental to all variants of logical atomism.

1. Complementation between atomic situations is not itself ultimate but is

grounded on a further complementation between nexuses or modes of configurations. Every atomic situation is a configuration of objects. The mode of that configuration is the nexus of the situation. In that variant of realist logical atomism that takes material properties and relations as constituents (objects) of configurations, the nexus of an atomic situation is called exemplification. Exemplification is not itself a material relationand perhaps is not a relation at all. (It is syncategorematic in so far as it is not indicated by any type of bound variable of the formal ontology.) Accordingly, in this variant of realist logical atomism, complementation requires that there be two exemplification nexuses, each the complement of the other, as the modes of all configurations having the same number of constituents. 
/) In In nominalist logical atomism, however, material properties and relations are not constituents of configurations but are rather the very modes of configurations themselves. That is, in nominalist logical atomism, material properties and relations, and only such, are nexuses. This, incidentally, seems to be the view of the Tractatus itself (cf. Sellars, "Naming and Saying," ([NAS]). Accordingly, complementation here consists in there being a distinct complement for each material property or relation. The complement of a material property or relation, it should be noted, is not a complex property or relation but is itself a simple material property or relation produced by a configuration of objects. For, of course, the independence of noncomplementary atomic situations excludes there being complex material properties or relations in logical atomism. That is, such complexity could be produced only by compounded configurations of objects; and in logical atomism it is precisely the ontological efficacy of compounded configurations over and above the efficacy of their atomic component configurations that is denied. It is only with respect to complementation that such compounding of configurations is not necessary, and, indeed, it is explicitly ruled out in our present variant of logical atomism.

6 Finally, there is a variant of realist logical atomism in which material properties and relations are not objects or constituents of configurations, but, as in nominalist logical atomism they are themselves nexuses or modes of configurations. This variant is realist, however, and not nominalistic in so far as material properties and relations are indicated by bindable predicate variables. Here, as in nominalism, we require that there be a simple material property or relation that is the complementary nexus of any given (simple) material property or relation.

Now it is noteworthy that the logical structure of the world as characterized by these different types of atomism differs significantly from characterization to characterization. This difference is not present, however, on the level of analysis dealing solely with propositional connectives and with which we are presently concerned. We mark the differences here only so as not to leave the reader uninformed about the nature of complementation between atomic situations.

Within the context of these remarks, we now climb a model-theoretic ladder that we will later throw away. Where $\mathscr{P}=\langle\mathrm{S}, \mathrm{f}\rangle$, we say that $\mathscr{\mathscr { P }}$ is an abstract logical space if $S$ is a set, empty or otherwise, and $f$ is a mapping from $S$ onto $S$ such that for each $s \in S, f(s) \neq s$ and $f(f(s))=s$. Infor- 
mally, $\mathrm{S}$ represents a space of atomic situations and $\mathrm{f}$ is the complementation operation between the situations in that space. In addition, we shall say that $\mathbf{A}$ is a possible world of $\mathscr{P}$, in symbols $\mathbf{A} \in \mathrm{W}(\mathscr{P})$, if $\mathbf{A} \subseteq S$, and for each $s \in S$, either $s \in A$ or $f(s) \epsilon A$, but not both.

Finally, we note that in real as opposed to abstract logical space, one possible world is the (real) world, namely, the totality of existing atomic situations. Other worlds are "unreal" in that some, if not all, of the atomic situations constituting them do not exist (in the real world). In logical atomism, possible-existence and possible-nonexistence are not material "properties" of atomic situations', though of course existence and (in our present variant) nonexistence are. That is, in an appropriate sense, existence (being-the-case) and nonexistence (being-not-the-case) are to atomic situations as material properties are to objects. But existence and nonexistence are the only such material "properties" of atomic situations. For possible-existence and possible-nonexistence are to atomic situations as internal properties (e.g., being-an-object, or being-self-identical) are to objects. And in logical atomism, as we have said in our introductory remarks, internal properties, be they of objects or of states of affairs, are formal and not material in nature. Of course, this is also a fundamental thesis common to all variants of logical atomism.

\section{Elementary Propositions and Complementation}

In turning to syntax we shall assume the availability of an indefinite number of elementary proposition signs.(Satzzeichen). We realize that a propositional sign is not itself a proposition, since "a proposition is a propositional sign in its projective relation to the world" ([TR] 3.12). But having hereby paid our dues in noting that we are ascending a Tractarian ladder, we shall for convenience usually refer to such signs simply as elementary propositions. Of course in contexts where the distinction is important, we shall speak of propositional signs, and distinguish these from the propositions expressed by such signs.

Mirroring our ontological assumption of a complementation between atomic situations, we assume that associated with each elementary proposition there is another elementary proposition called its complement. Naturally, an elementary proposition is to be both distinct from its com- 
plement and identical with the complement of its complement. Moreover, the correlation of complements is presumed to be effective in the sense that there exists a finite decision procedure by which we can decide whether any two elementary propositions are complementary or not. We shall use the bar-notation, '-', to indicate this effective complementation. Thus, where $\mathrm{p}$ is an elementary proposition, $\overline{\mathrm{p}}$ is also an elementary proposition, $\overline{\mathrm{p}} \neq \mathrm{p}$ and $\overline{\bar{\rho}}=\mathrm{p}$.

By a logical space of elementary propositions we understand an abstract logical space $\langle\mathrm{S}, \mathrm{f}\rangle$, where $\mathrm{S}$ is a set of elementary propositions and $f$ is the effective complementation correlation restricted to $S$. We note that in such a logical space different elementary propositions represent different atomic situations. We shall assume that our syntactical framework is sufficiently rich so that all metatheoretic considerations of logical space dealing solely with the formal properties of propositional connectives can be confined to logical spaces of elementary propositions. We assume in this way that the limit of empirical reality "makes itself manifest in the totality of elementary propositions." ([TR] 5.5561)

\section{Propositional Connectives as Punctuation Marks}

As primitive logical constants we shall use $\wedge, \vee, \square$ and $\diamond$ for the conjunction, disjunction, necessity and possibility signs, respectively. Where $\mathscr{P}$ is a logical space of elementary propositions, we take the set of propositions of $\mathscr{S}$ to be the smallest set $\mathrm{K}$ containing all the elementary propositions of $\mathscr{Y}$ and such that $\square \varphi, \diamond \varphi,(\varphi \wedge \psi),(\varphi \vee \psi)$ are in $\mathrm{K}$ whenever $\varphi$ and $\psi$ are in $\mathrm{K}$. (We remind the reader that a proposition of $\mathscr{S}$ is not a Tractarian proposition but rather a Tractarian propositional sign.) We shall occasionally use other constants such as $\rightarrow$ and $\leftrightarrow$ for the material conditional and biconditional signs. These signs and propositions containing them belong to an abbreviatory surface grammar generated from the ontological grammar of logical atomism by abbreviatory transformations. Any such sign that is not self-dual may be added to that grammar so long as its dual is also added. ${ }^{2}$

We note that the negation and affirmation signs, $\sim$ and $\approx$, respectively, are self-dual (and contradual ${ }^{3}$ to each other) and therefore will not be allowed to represent propositional operations of the ontological grammar. The reason for this exclusion is that such signs, in so far as they rep- 
resent existence (being-the-case) and nonexistence (being-not-the-case), are not really propositional connectives or logical constants in (our variant of) logical atomism after all. For, as noted, existence and nonexistence are material and not formal or internal "properties" of (atomic) situations. But in logical atomism, propositional connectives, and logical constants in general, do not represent real material operations. "Truth-functions are not material functions" ([TR] 5.44). "Signs for logical operations are punctuation-marks" ([TR] 5.4611). That is, propositional connectives represent only internal or formal "properties" and "relations" of propositions.

Our exclusion from the ontological grammar of all self-dual connectives is similarly motivated. For, in an appropriate sense, all such connectives reduce to either the negation or affirmation signs. ${ }^{4}$ That is, as an invariant syntactic-semantic rule, self-dual connectives when applied to propositions representing real (atomic) situations result in propositions that represent real (atomic) situations, and in that regard such connectives would indicate the existence of material truth-functions. Such truthfunctions, as noted, are excluded from the ontology of logical atomism.

Nevertheless, it is convenient to include the negation sign within our abbreviatory surface grammar. We do so by the following recursion: $\sim p$ $=\overline{\mathrm{p}}$ where $\mathrm{p}$ is an elementary proposition (and therefore $\sim \sim \mathrm{p}=\sim \overline{\mathrm{p}}=$ $\overline{\bar{\rho}} \mathrm{p}=\mathrm{p}) ; \sim(\varphi \wedge \psi)=(\sim \varphi \vee \sim \psi) ; \sim(\varphi \vee \psi)=(\sim \varphi \wedge \sim \psi) ; \sim \square \varphi=$ $\diamond \sim \varphi ;$ and $\sim \diamond \varphi=\square \sim \varphi^{S}$.

\section{Semantic Ascent/or Was This Trip Really Necessary?}

We now turn to the semantic rungs of our ladder. Where $\mathscr{H}$ is a logical space of elementary propositions and $\mathbf{A} \in \mathbb{W}(\mathscr{Y})$, we recursively characterize truth in the world $\mathbf{A}$ (with respect to the space $\mathscr{Y}$ ) as follows: (1) if p is an elementary proposition of $\mathscr{Y}$, then $\mathrm{p}$ is true in $\mathbf{A}$ (with respect to $\mathscr{S})$ iff $\mathrm{p} \epsilon \mathbf{A}$; and if $\varphi, \psi$ are propositions of $\mathscr{Y}$, then $(2)(\varphi \wedge \psi)$ is true in $\mathbf{A}$ (with respect to $\mathscr{Y}$ ) iff both $\varphi$ and $\psi$ are true in $\mathbf{A}$ (with respect to $\mathscr{Y}) ;(3)(\varphi \vee \psi)$ is true in $\mathbf{A}$ (with respect to $\mathscr{H}$ ) iff either $\varphi$ or $\psi$ is true in $\mathbf{A}$ (with respect to $\mathscr{Y})$; (4) $\square \varphi$ is true in $\mathbf{A}$ (with respect to $\mathscr{Y}$ ) iff for each $\mathbf{B} \in \mathrm{W}(\mathscr{Y}), \varphi$ is true in $\mathbf{B}$ (with respect to $\mathscr{P}$ ); and (S) $\diamond \varphi$ is true in $\mathbf{A}$ (with respect to $\mathscr{Y}$ ) iff for some $\mathbf{B} \epsilon \mathbb{W}(\mathscr{Y}), \varphi$ is true in $\mathbf{B}$ (with respect to $\mathscr{Y}$ ).

We say that a proposition of a logical space is valid in that space if it is 
true in every world of that space. In addition, a proposition is logically true if it is valid in every logical space of which it is a proposition. We note that a proposition is valid in a logical space if it is necessary in some (any) world of that space. That is, the semantical property of validity in a logical space as applied to propositional signs concurs with the internal "property" of logical necessity as applied to the propositions expressed by those propositional signs. By means of the following lemma, we see that at least on the level of analysis dealing solely with propositional connectives this also holds for logical truth. That is, a propositional sign is logically true iff the proposition expressed by that sign is logically necessary (in any world of any logical space of which it is a proposition). Accordingly, for this level of logical analysis, we do not need to resort to semantic ascent and station ourselves outside the world in order for a proposition to "show" (zeigen) its formal or internal "properties."

LEmma 1: If $\mathscr{Y}, \mathscr{S}^{\prime}$ are logical spaces of elementary propositions, A $\boldsymbol{\epsilon}$ $\mathrm{W}(\mathscr{Y}), \mathbf{A}^{\prime} \in \mathrm{W}\left(\mathscr{S}^{\prime}\right), \mathrm{p}_{\mathrm{o}}, \ldots, \mathrm{p}_{\mathrm{n}-1}$ are all the distinct elementary propositions occurring in $\varphi$, where $\varphi$ is a proposition of both $\mathscr{S}$ and $\mathscr{S}^{\prime \prime}$, and, for each $\mathrm{k}$ $<\mathrm{n}, \mathrm{p}_{\mathrm{k}} \in \mathbf{A}$ iff $\mathrm{p}_{\mathrm{k}} \in \mathbf{A}^{\prime}$, then $\varphi$ is true in $\mathbf{A}$ (with respect to $\mathscr{\mathscr { S }}$ ) iff $\varphi$ is true in $\mathbf{A}^{\prime}$ (with respect to $\left.\mathcal{S}^{\prime \prime}\right){ }^{6}$

The proof of lemma 1 is by induction on the structure of $\varphi$. In case $\varphi$ is an elementary proposition, the conclusion is an immediate consequence of the lemma's hypothesis. If $\varphi$ is a conjunction or disjunction, the conclusion follows directly from the inductive hypothesis. Suppose $\varphi$ is of the form $\square \psi$ and true in $\mathbf{A}$. We want then to show $\square \psi$ is true in $\mathbf{A}^{\prime}$. By reductio, suppose $\mathbf{B}^{\prime} \in \mathbb{W}\left(\mathscr{S}^{\prime}\right)$ and that $\psi$ is not true in $\mathbf{B}^{\prime}$. Let $\mathbf{B}$ be any world of $\mathscr{S}$ such that for all $\mathrm{k}<\mathrm{n}, \mathrm{p}_{\mathrm{k}} \in \mathbf{B}$ iff $\mathrm{p}_{\mathrm{k}} \in \mathbf{B}^{\prime}$. (We note that there must be at least one such world of $\mathscr{Y}$ since the notion of all possible worlds of $\mathscr{Y}$ has no restrictions or secondary interpretations placed upon it in our semantics. E.g. let $B=\left\{p_{k}: k<n\right.$ and $\left.p_{k} \in B^{\prime}\right\}, C=\{q \in A: \bar{q} \notin B\}$ and let $B=B$ $\cup \mathrm{C}$. Then it is easily seen that $\mathbf{B} \epsilon \mathrm{W}(\mathscr{P})$ and, for all $\mathrm{k}<\mathrm{n}, \mathrm{p}_{\mathrm{k}} \in \mathbf{B}$ iff $\mathrm{p}_{\mathrm{k}} \epsilon$ $\mathbf{B}^{\prime}$.) But since $\square \psi$ is true in $\mathbf{A}, \psi$ is true in $\mathbf{B}$, and therefore, by the inductive hypothesis, $\psi$ is true in $\mathbf{B}^{\prime}$, which, by assumption is impossible. Accordingly, if $\square \psi$ is true in $\mathbf{A}$ (with respect to $\mathscr{P}$ ), then $\square \psi$ is true in $\mathbf{A}^{\prime}$ (with respect to $\mathscr{P}^{\prime}$ ). Proof of the converse is entirely similar. Finally, suppose $\varphi$ is of the form $\diamond \psi$ and true in $\mathbf{A}$. We want now to show that $\diamond \varphi$ is 
true in $\mathbf{A}^{\prime}$. By definition, $\psi$ is true in some $\mathbf{B} \epsilon \mathbb{W}(\mathscr{P})$. Let $\mathbf{B}^{\prime}$ be any world of $\mathscr{S}^{\prime}$ such that for all $k<\mathrm{n}, \mathrm{p}_{\mathrm{k}} \in \mathbf{B}^{\prime}$ iff $\mathrm{p}_{\mathrm{k}} \in \mathbf{B}$. Again, for the same reason as in the previous case, there must be such a world of $\mathscr{S}^{\prime}$. Then, by the inductive hypothesis, $\psi$ is true in $\mathbf{B}^{\prime}$, and therefore $\diamond \psi$ is true in $\mathbf{A}^{\prime}$. The argument to show that $\diamond \psi$ is true in $\mathbf{A}^{\prime}$ only if it is true in $\mathbf{A}$ is entirely simi$\operatorname{lar}($ Q.E.D.).

THEOREM 1: A proposition is logically true iff it is valid in some logical space (of which it is a proposition). ${ }^{?}$

The proof in one direction is trivial. For $\varphi$ is a proposition only if it is a proposition of some logical space, and therefore if $\varphi$ is logically true, then $\varphi$ is valid in that logical space. For the converse direction, suppose that $\varphi$ is valid in some logical space $\mathscr{Y}$ of which it is a proposition, and suppose further that $\mathscr{S}^{\prime \prime}$ is also a logical space of which $\varphi$ is a proposition. It suffices to show that $\varphi$ is valid in $\mathscr{Y}^{\prime}$. Assume, for reductio, that $\mathbf{A}^{\prime} \epsilon \mathrm{W}\left(\mathscr{Y}^{\prime}\right)$ and that $\varphi$ is not true in $\mathbf{A}^{\prime}$ (with respect to $\mathscr{Y}^{\prime}$ ). Let $\mathrm{p}_{0}, \ldots, \mathrm{p}_{\mathrm{n}-1}$ be all the distinct elementary propositions occurring in $\varphi$ that are true in $\mathbf{A}^{\prime}$, and let $\mathrm{q}_{\mathrm{o}}, \ldots, \mathrm{q}_{\mathrm{m}-1}$ be all the distinct elementary propositions occurring in $\varphi$ that are not true in $\mathbf{A}^{\prime}$. And finally, let $\mathbf{A}$ be any world of $\mathscr{P}$ that contains $\mathrm{p}_{\mathrm{o}}, \ldots, \mathrm{p}_{\mathrm{n}-1}$ and the complements of $\mathrm{q}_{\mathrm{o}}, \ldots, \mathrm{q}_{\mathrm{m}-1}$. (As in the proof of the preceding lemma, there must be such a world since 'all possible worlds (of $\mathcal{P})^{\prime}$ has its standard and not a secondary or restricted meaning.) Accordingly, by lemma $1, \varphi$ is not true in $\mathbf{A}$ (with respect to $\mathscr{S}$ ). But that is impossible since by assumption $\varphi$ is valid in $\mathscr{Y}$. (Q.E.D.)

We might note here that by the above theorem a propositional sign expressing a proposition of real logical space is logically true iff the proposition expressed by that sign is logically necessary (in the real world). That is, a propositional sign $\varphi$ of real logical space is logically true iff $\square \varphi$ is true simpliciter. Similarly, where the logical falsity of a propositional sign is the logical truth of its dual, and its contingency is its being neither logically true nor logically false, a propositional sign $\varphi$ of real logical space is logically false or contingent, respectively, iff $\square \sim \varphi$ or $(\diamond \varphi \wedge \diamond \sim \varphi)$, respectively, are true simpliciter. Here we see the proper concurrence of semantic properties of propositional signs with internal "properties" of the propositions expressed by those signs. And of course the same concurrence holds regarding semantic relations between propositional signs and 
internal "relations" between propositions: e.g., the semantic relation of entailment (on this level of analysis) concurs with the internal "relation" of strict implication. Accordingly, as already noted, for this level of logical analysis we do not need to resort to semantic ascent and station ourselves outside the world in order for a proposition to "show" (zeigen) its formal or internal "properties" and "relations."

\section{Zeigen versus Sagen}

We should not conclude from the above that the truth in the (real) world of $\square \varphi, \square \sim \varphi$, or $(\diamond \varphi \wedge \diamond \sim \varphi)$ "says" (sagt) rather than merely "shows" (zeigt) that the proposition expressed by $\varphi$ has the internal "property" of being logically necessary, impossible, or contingent, respectively. Nor similarly, should we conclude that the truth of $\square(\varphi \rightarrow \psi)$ "says" rather than "shows" that the internal "relation" of strict implication holds between (the propositions expressed by) $\varphi$ and $\psi$. Such could be "said" only if $\square$ and $\diamond$ represented real material "properties" of propositions and thereby real material "properties" of situations or states of affairs. And of course if $\square$ and $\diamond$ were to (purport to) represent material "properties," then they would indeed be quite alien to logical atomism after all. For in logical atomism, as we have noted, only existence (being-the-case) and (in our variant) nonexistence (being-not-the-case) are real material "properties" of (atomic) situations.

Now our position here is not that $\square$ and $\diamond$ are modal operators and therefore not predicates designating properties of objects. "Here the shifting use of the word 'object' corresponds to the shifting use of the words 'property' and 'relation'" ([TR], 4.123). That is, our position rather is that the internal "properties" of propositions represented by $\square$ and $\diamond$ are merely formal and not real material "properties" of propositions or the situations represented by propositions. And in that regard, to use the Tractarian terminology, the "ascription" of such "properties" is not a genuine "saying" but really only a "showing." And "what can be shown, cannot be said" ([TR], 4.1212). Accordingly, if this position is correct, the inclusion of $\square$ and $\diamond$ as primitive connectives of the ontological grammar would not violate logical atomism's exclusion of real material internal properties (of either type of "object").

But more than that $\square$ and $\diamond$ represent formal, and not material, inter- 
nal "properties," it is also our claim that these internal "properties" cannot be adequately "shown" (gezeigt) by propositions without the introduction of $\square$ and $\diamond$ into the ontological grammar of logical atomism. And this is so, moreover, precisely because what is necessary, impossible or contingent in one world (of a logical space), e.g., the real world (of real logical space), is therefore also necessary, impossible or contingent, respectively in all other worlds (of that logical space)—where 'all' has its standard and not a secondary or restricted interpretation. That is, it is precisely because of this standard use of 'all' (and 'some') in the truth-clause of $\square$ (and $\diamond$ ) that the invariance of semantical properties of propositional signs concurs (on this level of analysis) with the invariance of internal "properties" of propositions. And since the concurrence of these invariance conditions is after all just about as appropriate an explication of 'formal' (in the traditional sense) as might be given, we return to our claim that $\square$ and $\diamond$ represent formal and not material "properties" of propositions. We proceed in the justification of our position by examining its claims in the opposite order presented.

In justification of our last claim regarding the standard meaning of 'all', let us consider the consequences of attaching a secondary or restricted meaning to the notion(s) of all (and some) possible world(s) (of a given logical space). Where $\mathscr{S}$ is a logical space of elementary propositions, $\mathrm{K} \subseteq \mathrm{W}(\mathscr{S})$, and $\mathbf{A} \epsilon \mathrm{K}$, we recursively redefine truth in $\mathbf{A}$ (with respect now to $K$ as well as to $\mathscr{P}$ ) as follows: (1) where p is an elementary proposition of $\mathscr{P}, \mathrm{p}$ is true in $\mathbf{A}$ (with respect to $\mathrm{K}$ and $\mathscr{S}$ ) iff $\mathrm{p} \in \mathbf{A}$; and where $\varphi, \psi$ are propositions of $\mathscr{S},(2),(\varphi \wedge \psi)$ is true in $\mathbf{A}$ (with respect to $\mathrm{K}$ and $\mathscr{\varphi})$ iff $\varphi$ and $\psi$ are true in $\mathbf{A}$ (with respect to $K$ and $\mathscr{S}) ;(3)(\varphi \vee \psi)$ is true in $\mathbf{A}$ (with respect to $\mathrm{K}$ and $\mathscr{P}$ ) iff either $\varphi$ or $\psi$ is true in $\mathbf{A}$ (with respect to $\mathrm{K}$ and $\mathscr{Y}$ ); (4) $\square \varphi$ is true in $\mathbf{A}$ (with respect to $\mathrm{K}$ and $\mathscr{S}$ ) iff $\varphi$ is true (with respect to $\mathrm{K}$ and $\mathscr{S}$ ) in all worlds (of $\mathscr{P}$ ) that are in $\mathrm{K}$; and $(5) \diamond \varphi$ is true in $\mathbf{A}$ (with respect to $K$ and $\mathscr{P}$ ) iff $\varphi$ is true (with respect to $K$ and $\mathscr{S}$ ) in some world (of $\mathscr{P}$ ) that is in $\mathrm{K}$.

Clearly, the meaning of 'all possible worlds (of $\mathscr{P}$ )' (and its dual 'some possible world (of $\mathscr{P}$ )') has been given a secondary or restricted interpretation in this alternative characterization of semantic truth. We now consider similar alternatives to validity and logical truth. Where $\mathscr{S}$ and $\mathrm{K}$ are as above and $\varphi$ is a proposition of $\mathscr{P}$, then $\varphi$ is K-valid (in $\mathscr{P}$ ) if $\varphi$ is true (with respect to $\mathrm{K}$ and $\mathscr{S}$ ) in every world (of $\mathscr{P}$ ) that is in $\mathrm{K}$. And $\varphi$ is uni- 
versally valid if $\varphi$ is K-valid for any non-empty set K of worlds of any logical space of which $\varphi$ is a proposition. This notion of universal validity, incidentally, is essentially that of Kripke's in ["A Completeness Theory in Model Logic," ([ACT])]; and, therefore, as shown by Kripke, a proposition is universally valid iff it is a theorem of the modal propositional calculus $\mathrm{S} 5 .^{8}$

Now it is easily seen that every universally valid proposition is logically true. ${ }^{9}$ But, on the other hand, not every logically true proposition is universally valid. For example, where $\mathrm{p}$ is an elementary proposition of a logical space $\mathscr{P}$, there must, as a fundamental thesis of logical atomism, be a world $\mathbf{A}$ of $\mathscr{S}$ in which $\mathrm{p}$ is true and another world $\mathbf{B}$ of $\mathscr{S}$ in which $\mathrm{p}$ is false, i.e., in which $\sim \mathrm{p}$ is true. Accordingly, both $\diamond \mathrm{p}$ and $\diamond \sim \mathrm{p}$ are logically true. And that, of course, is exactly as it should be in logical atomism. But in regard to universal validity, there is always at least one set of worlds of $\mathscr{S}$, e.g., the unit set $\{\mathbf{B}\}$, in all the worlds of which $\mathrm{p}$ is not true. And, similarly, there is always at least one set of worlds of $\mathscr{S}$, e.g., the unit set $\{\mathbf{A}\}$, in all the worlds of which $\sim \mathrm{p}$ is not true. Therefore, both $\diamond \mathrm{p}$ and $\diamond \sim \mathrm{p}$ fail to be universally valid.

What the above illustrates is that the notion of validity in a set of worlds of a logical space-as opposed to validity in all the worlds of that space-introduces a type of necessity and possibility, and thereby strict entailment too, which while applying to a proposition in some worlds or sets of worlds of a logical space need not for that reason alone apply to that proposition in all other worlds or sets of worlds of that logical space. This type of internal "property" is not an invariant feature of a proposition throughout all the worlds of a logical space; and precisely for that reason this type of internal "property" is a material condition of the propositions to which it applies. And of course it is exactly the reality of such material, internal "properties" that is totally alien to logical atomism. ${ }^{10}$

A further point to note about universal validity, as opposed to logical truth, is that the substitution of any proposition-be that proposition a conjunction, disjunction, necessity or possibility, and of whatever complexity-for an elementary proposition in a universally valid proposition always results in a universally valid proposition. This is so because universal validity characterizes the theorems of $\mathrm{S} 5$ and substitution is known to hold in S5. That is, where $\varphi$ is a proposition and $\varphi\left[\begin{array}{l}\mathrm{p} \\ \psi\end{array}\right]$ is the result of replacing each occurrence of the elementary proposition $\mathrm{p}$ in $\varphi$ by an occur- 
rence of the proposition $\psi$ (of whatever complexity) and each occurrence of $\bar{p}$ by $\sim \psi$, then $\varphi$ is universally valid only if $\varphi\left[{ }_{\psi}^{\mathrm{P}}\right]$ is universally valid.

Now the fact that substitution of complex propositions for elementary propositions preserves universal validity indicates that if universal validity were taken as characterizing the logical structure or "scaffolding of the world" ([TR], 6.124), then there are complex situations or states of affairs in just the same sense of being (or 'there are') as there are atomic situations or states of affairs. Indeed, there need not even be atomic situations in the world, and elementary propositional signs would then represent only the deepest level of situational analysis attained thus far or for the purposes at hand.

In other words, if quantifiable propositional variables were allowedin which case of course the ontology represented would not be nominalistic-then the substitution rule would express itself in the fact that complex propositional signs are substituends of bound propositional variables. And, accordingly, as substituends for bound propositional variables an appropriate ontologically committal form of the comprehension principle for complex propositions (or situations represented by propositions) would then be provable. ${ }^{11}$ That is, in so far as the formal ontology would express its ontological commitments via its bound variables, complex states of affairs would be viewed as being as fundamentally real as atomic situations or states of affairs, even assuming the latter to be at all. And then of course these complex situations would have real material, internal "properties," as argued above. No doubt we are here caught up in ontological theses quite foreign to logical atomism.

Of course, in nominalistic formal ontologies only individual variables are bindable. Nominalism, in other words, is a form of categorial monism. Nevertheless, although they are not individuals (objects), atomic situations have in all variants of logical atomism an ontological status that is not shared by so called complex situations (all of which are ontologically resolvable into their atomic components). And it is in reference to this special ontological status that we have spoken of atomic situations as "real" or as having a mode of being.

This need not mean, however, that their mode of being is just as fundamental as that of objects and that they should be indicated by bindable propositional variables. Otherwise, of course, there can be no form of 
logical atomism which is genuinely nominalistic. No, instead of expressing its commitment via bound propositional variables, nominalistic (and, a fortiori, realist) logical atomism indicates its peculiar form of commitment to atomic situations and its lack of commitment to complex situations via a substitution rule that holds for elementary propositional signs but fails for complex propositional signs not logically equivalent to an elementary proposition. ${ }^{12}$

Naturally, this criterion is but a variation of the nominalist's standard gambit of reparsing the realist's references to different types of entities by means of references to different types of signs instead. And of course relative to this nominalistic criterion for commitment, universal validity is ruled out as a characterization of the logical structure of the world according to logical atomism. But then so is standard propositional logic, and so too standard predicate logic. For in standard logic the substitution rule applies to all propositions of whatever complexity. And therefore standard logic is just as inappropriate a formal representative of logical atomism as is any modal logic in which the substitution rule holds for all propositions. Substitution, with or without restrictions, is in effect the nominalist's means of expressing in his limited way what the realist expresses through corresponding universal instantiation principles with or without the same restrictions. And, in general, realist universal instantiation principles are equivalent to comprehension principles with the same restrictions but which represent ontological commitments in a more direct or obvious manner. And, of course in logical atomism, whether of the nominalist or realist variants, it is substitution and/or a universal instantiation or comprehension principle with restrictions of the sort described above that is required.

But now what of the substitution rule in the semantics of logical truth as characterized here? We note first that substitution of conjunctive propositions does not hold in general; e.g., the substitution of (q $\wedge \sim \mathrm{q})$ for $\mathrm{p}$ in the logically true proposition $\diamond \mathrm{p}$ results in the logically false proposition $\diamond(\mathrm{q} \wedge \sim \mathrm{q})$. Similarly, the substitution of disjunctive propositions also does not hold in general; e.g., the substitution of $(q \vee \sim q)$ for $\mathrm{p}$ in the logically true proposition $\diamond \sim \mathrm{p}$ results in the logically false proposition $\diamond \sim(\mathrm{q} \vee \sim \mathrm{q})$. Thus conjunctive and disjunctive propositional signs do not in general represent real (atomic) situations. More- 
over, the substitution of $\square \mathrm{q}$ and $\diamond \mathrm{q}$ for $\mathrm{p}$ in $\diamond \mathrm{p}$ and $\diamond \sim \mathrm{p}$, respectively, shows us that a similar observation holds for propositional signs beginning with $\square$ and $\diamond$.

It is noteworthy that in each of the above examples not only does substitution fail to preserve logical truth, but, in addition, it actually transforms a logical truth into a logical falsity. And in that sense these propositions "show" themselves to have the internal "property" of being complex, that is, as not being logically equivalent to an elementary proposition; and, accordingly, they "show" themselves in this sense as failing to represent a real (atomic) situation. The following lemma shows that this is the case for all genuinely complex propositions. And note, incidentally, that $\square$ and $\diamond$ with the proper "logico-syntactical employment" are essential to the proof of this lemma.

LEMMA 2: If $\psi$ is a proposition that is not logically equivalent to any elementary proposition, then there are a logically true proposition $\varphi$ and an elementary proposition p occurring in $\varphi$ such that $\varphi\left[\begin{array}{l}p \\ \psi\end{array}\right]$ is logically false.

For the proof of lemma 2, suppose $\psi$ is a proposition that is not logically equivalent to any elementary proposition, and let $\mathrm{p}$ be any elementary proposition not occurring in $\psi$. Then $\sim \square(\mathrm{p} \leftrightarrow \psi)$ is logically true; for otherwise, $\square(p \leftrightarrow \psi)$ would be true in some world of some logical space, in which case $\square(p \leftrightarrow \psi)$ would be valid in that space, and therefore, by theorem 1 , logically true, which by assumption is impossible. But $\sim \square(\mathrm{p} \leftrightarrow \psi)$, by abbreviatory transformations, reduces to $\diamond([\mathrm{p} \wedge \sim \psi] \vee$ $[\overline{\mathrm{p}} \wedge \psi]$ ), and of course substituting $\psi$ for $\mathrm{p}$ in this logically true proposition results in the logically false proposition $\diamond([\psi \wedge \sim \psi] \vee[\sim \psi \wedge \psi])$. (Q.E.D.)

In regard to showing that substitution of elementary propositions in logically true propositions always results in logically true propositions, we utilize the following definition and subsequent lemma. Where $\mathscr{P}$ is a logical space of elementary propositions containing $\mathrm{p}$ and $\mathrm{q}$, and $\mathbf{A} \boldsymbol{\epsilon}$ $\mathrm{W}(\mathscr{Y})$, we take $\mathbf{A}\left({ }_{q}^{\mathrm{p}}\right)$ to be that unique world of $\mathscr{S}$ determined as follows:

$$
\mathbf{A}\left({ }_{\mathrm{q}}^{\mathrm{p}}\right)=\left\{\begin{array}{l}
\mathbf{A} \text { if } \mathrm{p}, \mathrm{q} \in \mathbf{A} \text { or } \overline{\mathrm{p}}, \overline{\mathrm{q}} \boldsymbol{\epsilon} \mathbf{A} \\
(\mathbf{A}-\{\mathrm{p}\}) \cup\{\overline{\mathrm{p}}\} \text { if } \mathrm{p}, \overline{\mathrm{q}} \boldsymbol{\epsilon} \\
(\mathbf{A}-\{\overline{\mathrm{p}}\}) \cup\{\mathrm{p}\} \text { if } \overline{\mathrm{p}}, \mathrm{q} \in \mathbf{A}
\end{array}\right.
$$


LEMMA 3: If $\mathscr{S}$ is a logical space of elementary propositions containing $\mathrm{p}$ and $\mathrm{q}, \varphi$ is a proposition of $\mathscr{S}$ in which neither q nor its complement occurs, and $\mathbf{A} \in \mathrm{W}(\mathscr{S})$, then $\varphi\left[{ }_{\mathrm{q}}^{\mathrm{P}}\right]$ is true in $\mathbf{A}$ (with respect to $\mathscr{S}$ ) iff $\varphi$ is true in $\mathbf{A}\left({ }_{\mathrm{q}}^{\mathrm{P}}\right)$ (with respect to $\mathscr{P}$ ).

The proof for lemma 3 is by strong induction on the number of occurrences of connectives in $\varphi$ and then by an inductive argument on the structure of $\varphi$. Suppose then that the number of connectives occurring in $\varphi$ is $n$ and that lemma 3 holds for all propositions with a fewer number of occurrences of connectives. If $\varphi$ is itself an elementary proposition, then lemma 3 follows directly from the definition of $\mathbf{A}\left({ }_{\mathrm{q}}^{\mathrm{P}}\right)$. If $\varphi$ is a conjunction or disjunction, then lemma 3 holds by the inductive hypothesis regarding the conjuncts or disjuncts of $\varphi$. Suppose $\varphi$ is of the form $\square \psi$ and that $\square \psi\left[\left[_{q}^{p}\right]\right.$ is true in $\mathbf{A}$. Then to show that $\square \psi$ is true in $\mathbf{A}(\underset{q}{\mathrm{p}})$ it suffices to show that $\psi$ is true in every world of $\mathscr{P}$.

Assume, accordingly, that $\mathbf{B} \epsilon \mathrm{W}(\mathscr{P})$. We note that since, by hypothesis, neither q nor its complement occurs in $\varphi$, then neither q nor its complement occurs in $\psi$, and therefore $\psi\left[{ }_{q}^{p}\right]\left[\left[_{p}^{q}\right]=\psi\right.$. Moreover, since $\psi$ has fewer than $\mathrm{n}$ many occurrences of connectives, then by the strong inductive hypothesis, $\psi\left[{ }_{q}^{p}\right]\left[{ }_{p}^{q}\right]$ is true in $\mathbf{B}$ iff $\psi\left[{ }_{q}^{p}\right]$ is true in $\mathbf{B}\left({ }_{p}^{q}\right)$. But, by assumption $\psi\left[\left[_{q}^{p}\right]\right.$ is true in every world of $\mathscr{S}$, and therefore $\psi\left[{ }_{q}^{p}\right]\left[{ }_{p}^{q}\right]$, that is, $\psi$, is true in $\mathbf{B}$, which was to be shown. If, conversely, $\square \psi$ is true in $\mathbf{A}\left({ }_{q}^{p}\right)$, then $\psi$ is true in every world of $\mathscr{S}$, and therefore by the inductive hypothesis, $\psi\left[\left[_{\mathrm{q}}^{\mathrm{p}}\right]\right.$ is true in every world of $\mathscr{P}$; and, $\square \psi[\mathrm{q}$ 学] is accordingly true in $\mathbf{A}$. A similar argument applies in case $\varphi$ is of the form $\diamond \psi$. (Q.E.D.)

LEMMA 4: If $\mathrm{p}, \mathrm{q}$, are elementary propositions and $\varphi$ is a logically true proposition in which neither $q$ nor its complement occurs, then $\varphi\left[{ }_{q}^{p}\right]$ is also logically true. ${ }^{13}$

Lemma 4 follows directly from lemma 3 . For suppose the hypothesis of lemma 4 is true but that $\varphi\left[{ }_{q}^{p}\right]$ is not logically true. Then there is a logical space $\mathscr{S}$ of elementary propositions containing $\mathrm{p}$ and $\mathrm{q}$ and of which $\varphi[\mathrm{p}]$ is a proposition that is not true in some world $\mathbf{A}($ of $\mathscr{P}$ ). But then, by lemma 3, $\varphi$ is not true in $\mathbf{A}\left({ }_{q}^{(\mathrm{P}}\right)$, which is impossible since $\varphi$ is logically true. (Q.E.D.) 
We conclude from these observations that the semantics for logical truth as characterized here is completely in line with our proposed criterion for nominalistic commitment in logical atomism. The same observations apply a fortiori for realist logical atomism. Accordingly, unlike the semantics for universal validity, $\square$ and $\diamond$ here do not represent real material "properties" of situations. Moreover, $\square$ and $\diamond$, with their proper "logico-syntactical employment" are essential for the representation of formal, internal "properties" basic to the ontology of logical atomism.

\section{The Propositional Modal Calculus S13}

Finally, in the way of a brief description of a calculus characteristic of this semantics, let us consider the system $S 13$ formulated by David Kaplan. ${ }^{14}$ The axioms and rules of $\mathrm{S} 13$ are just the axioms and rules of $\mathrm{S} 5$ supplemented with the following schema: if $\varphi$ is a modal free proposition that is not tautologous, then $\diamond \sim \varphi$ is an axiom of $\$ 13$. Clearly, every instance of this schema is logically true since every logical space of which $\varphi$ is such a modal free proposition contains a world in which $\varphi$ is false. Also, since S5 is characteristic of universal validity and every universally valid proposition is logically true, every axiom of S13 is therefore logically true. Moreover, because modus ponens and modal generalization (the rules of S13) preserve logical truth, every theorem of $\mathrm{S} 13$ is therefore logically true. That is, S13 is sound with respect to logical truth as characterized here.

The following lemmas are of some interest in their own right as well as being useful in proving our completeness theorem. We understand a proposition to be modally closed if each occurrence of each of its component elementary propositions lies within the scope of an occurrence of $\square$ or $\diamond$.

LEMMA 5: In S13, every proposition is provably equivalent to a modal free proposition.

The proof for lemma 5 is by induction on the structure of an arbitrary proposition $\varphi$. Where $\varphi$ is an elementary proposition, lemma 5 holds trivially. Where $\varphi$ is a conjunction or disjunction, lemma 5 holds for $\varphi$ by the inductive hypothesis. Suppose that $\varphi$ is of the form $\square \psi$ or $\diamond \psi$. Then, by 
the inductive hypothesis, there is a modal free proposition $\chi$ such that $\overline{513}$ $(\psi \leftrightarrow \chi)$ and therefore by modal generalization and the distribution of $\square$ over $\leftrightarrow, \overline{\mathrm{S} 13}(\square \psi \leftrightarrow \square \chi)$ and $\bar{s} \overline{\mathrm{s}} \overline{\mathrm{s}}\left(\nabla_{\psi} \psi \leftrightarrow \diamond \chi\right)$. We note that if $\chi$ is a tautology, then both $\square \chi$ and $\diamond \chi$ are theorems of S13, and therefore so are $\square \psi$ and $\diamond \psi$, in which case $\varphi$ is provably equivalent to the modal free proposition ( $\mathrm{p} \vee \sim \mathrm{p}$ ). On the other hand, if $\sim \chi$ is a tautology, then both $\sim \diamond \chi$ and $\sim \square \chi$ are theorems of $S 13$, and therefore so are $\sim \square \psi$ and $\sim \diamond \psi$, in which case $\varphi$ is provably equivalent to $(\mathrm{p} \wedge \sim \mathrm{p})$. Finally, if neither $\chi$ nor $\sim \chi$ are tautologies, then both $\diamond \chi$ and $\diamond \sim \chi$ are axioms of $\$ 13$, and therefore $\diamond \psi$ and $\sim \square \psi$ are theorems, in which case $\varphi$ is provably equivalent to (p $\wedge \sim \mathrm{p}$ ) if $\varphi$ is $\square \psi$, and provably equivalent to ( $\mathrm{p} \vee \sim \mathrm{p}$ ) if $\varphi$ is $\diamond$ $\psi$. (Q.E.D.)

LEMMA 6: If $\varphi$ is a modally closed proposition, then either $\overline{513} \varphi$ or $\grave{S 13} \sim \varphi$.

The proof for lemma 6 utilizes lemma 5. For, by lemma 5, there is a modal free proposition $\psi$ such that $\overline{s 13}(\varphi \leftrightarrow \psi)$, and therefore by modal generalization and distribution of $\square$ over $\leftrightarrow, \widehat{S 13}(\square \varphi \leftrightarrow \square \psi)$. We note however that in $S 5$, if $\varphi$ is modally closed, then $\overline{S 5}^{-}(\varphi \leftrightarrow \square \varphi)$; and, there-

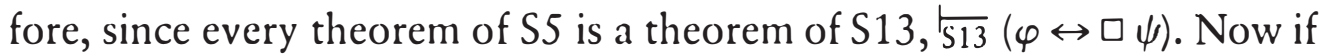
$\psi$ is a tautology, then $\overline{S 13} \square \psi$ and therefore $\overline{S 13} \varphi$. But if $\psi$ is not a tautology, then $\diamond \sim \psi$ is an axiom of $S 13$, in which case $\overline{s 13} \sim \varphi$. (Q.E.D.)

Although lemma 5 is used in the proof of lemma 6, the philosophical rationale for lemma 5 is easily seen via lemma 6 . For according to lemma 6 and the fact that $\mathrm{S} 13$ is sound, each modally closed component proposition of a given proposition is either logically true, and therefore replaceable by a modal free tautology, or logically false, and therefore replaceable by the dual of a modal free tautology; and, accordingly, each proposition is ontologically resolvable into its component elementary propositions not occurring in the scope of a modal operator. Lemma 6 itself, on the other hand, is exactly what one would expect (on this level of analysis) if $\square$ and $\diamond$ represent, as we have argued they do, formal, and not material, internal "properties" of propositions or situations represented by these propositions. And in that regard, lemma 6 is a further substantiation of our position.

For proving completeness, we say as usual that a set $\mathrm{K}$ of propositions 
is maximally consistent (in S13) if $\mathrm{K}$ is consistent (in S13) but results in an inconsistent set when it is supplemented with any proposition all of the elementary components of which, or their complements, occur in propositions in $\mathrm{K}$. Where $\mathrm{K}$ is such a maximally consistent set, we take $\mathscr{S}_{\mathrm{K}}$ to be the logical space of elementary propositions that occur in some proposition in K. Moreover, we take $A_{K}$ to be that world of $\mathscr{S}_{\mathrm{K}}$ such that for each elementary proposition $\mathrm{p}, \mathrm{p} \in \mathbf{A}_{\mathrm{K}}$ iff $\mathrm{p} \in \mathrm{K}$. The following lemma leads directly to our completeness theorem.

LEMMA 7: If $\mathrm{K}$ is a set of propositions that is maximally consistent in $\mathrm{S} 13$ and $\varphi$ is a proposition of $\mathscr{Y}_{\mathrm{K}}$, then $\varphi$ is true in $\mathbf{A}_{\mathrm{K}}$ (with respect to $\mathscr{P}_{\mathrm{K}}$ ) iff $\varphi \epsilon$ $\mathrm{K}$.

The proof of lemma 7 is by induction on the structure of $\varphi$. If $\varphi$ is an elementary proposition, then lemma 7 holds by definition of $\mathbf{A}_{K}$; if $\varphi$ is a conjunction or disjunction, then lemma 7 holds for $\varphi$ by the inductive hypothesis and the fact that a conjunction is in a maximally consistent set iff both conjuncts are, and a disjunction is in such a set iff at least one disjunct is. If $\varphi$ is of the form $\square \psi$ or $\diamond \psi$, then $\varphi$ is modally closed, and therefore, by lemma 6, either $\overline{s 13} \varphi$ or $\overline{s 13} \sim \varphi$.

Suppose first that $\stackrel{5}{S 13} \varphi$. Then $\varphi \in \mathrm{K}$, since otherwise $\mathrm{K}$ would be inconsistent. Moreover, since every theorem of $S 13$ is logically true, $\varphi$ is logically true and, therefore, true in $\mathbf{A}_{K}$. Accordingly, $\varphi \in \mathrm{K}$ and $\varphi$ is true in $\mathbf{A}_{K}$, and therefore $\varphi$ is true in $\mathbf{A}_{\mathrm{K}}$ iff $\varphi \in \mathrm{K}$. Suppose, on the other hand, $\overline{\mathrm{s} 13}$ $\sim \varphi$; then $\sim \varphi \epsilon \mathrm{K}$ and $\sim \varphi$ is logically true and, therefore, true in $\mathbf{A}_{K}$, that is, $\varphi \notin \mathrm{K}$ and $\varphi$ is not true in $\mathbf{A}_{\mathrm{K}}$, and accordingly, $\varphi$ is true in $\mathbf{A}_{\mathrm{K}}$ iff $\varphi \in \mathrm{K}$. (Q.E.D.)

As usual, by Lindenbaum's lemma, every consistent set of propositions can be expanded to a maximally consistent set, and therefore, by lemma 7 , all the members of a consistent set of propositions are true in some one and the same world of a logical space of elementary propositions.

COMPLETENESS THEOREM: If $\mathrm{K}$ is a set of propcsitions that is consistent in S13, then there exists a logical space $\mathscr{P}$ and a world $\mathbf{A}$ of $\mathscr{Y}$ such that every proposition in $\mathrm{K}$ is true in $\mathbf{A}$ (with respect to $\mathscr{P}$ ).

COROLLARY: A proposition $\varphi$ is logically true iff $\overleftarrow{S 13} \varphi$. 
According to logical atomism, the principle of individuation for the worlds of a logical space is simply that every atomic situation that exists in the one exists in the other, i.e., worlds are identical when they coincide in their atomic facts. But, relative to the logistic system that is the determinant of (syntactical) consistency, and relative to the level of logical analysis dealing solely with propositional connectives, maximally consistent sets of propositions constitute as rich a description of a possible world as can consistently be given. Therefore, $\mathrm{S} 13$-and, by the completeness theorem, the semantics of logical truth as characterized here-is an appropriate formal representative on the propositional level of logical atomism only if in S13 maximally consistent sets of propositions are completely individuated by the elementary propositions that are their members. By lemma 8 , which is a direct consequence of lemma 7, this is indeed the case in S13.

LEMMA 8: If $\mathrm{K}, \mathrm{K}^{\prime}$ are sets of propositions that are maximally consistent in $\mathrm{S} 13$, and $\mathscr{S}_{\mathrm{K}}=\mathscr{S}_{\mathrm{K}^{\prime}}$, then $\mathrm{K}=\mathrm{K}^{\prime}$ iff $\mathbf{A}_{\mathrm{K}}=\mathbf{A}_{\mathrm{K}^{\prime}}$.

Thus, according to lemma 8 , worlds that are indiscernible in their atomic facts are indiscernible simpliciter. In particular, worlds indiscernible in their atomic facts are indiscernible in their modal facts. Such modal facts therefore can involve only formal, and not material, internal "properties" and "relations." Lemma 8, on the other hand, fails for S5 and other modal systems. In the formal ontologies containing such systems, $\square$ and $\diamond$ (purport to) represent internal "properties" with real differentiating material content. Such formal ontologies are ruled out as repesentatives of logical atomism. But S13 has passed the test in every way.

Notes

1 We shall use scare-quotes when speaking of what connectives represent as "properties" or "relations." We do so to mark a special philosophical use which is heuristically convenient in our informal discourse but which strictly speaking is ontologically misleading. We do not use scare-quotes when speaking of properties and relations of objects (since in this case it is not ontologically misleading), nor occasionally when speaking of objects and situations together.

2 The dual of a connective $\xi$ is that connective the truth-table for which is the result of 
transposing the principal column in the truth-table for $\xi$. (To transpose a column: interchange T's and F's and then turn the resulting column upside down.) A truth-tabular account of $\square$ and $\diamond$ that is equivalent to the metaphysically oriented semantics we shall give is to place a $T$ in the principal column for $\square(\diamond)$ iff the proposition to which it is affixed has a $\mathrm{T}$ in every (some) row of the table. Naturally, $\square$ and $\diamond$ are dual to each other.

3 Contraduality: turn the principal column upside down. Propositions are dual to one another if each is obtainable from the other by interchanging dual logical constants. They are contraduals if elementary propositions are interchanged with their complements.

4 For example, where $\left(p^{*} q\right)$ and $(p \ q)$ have the same truth-tables as $(p \wedge[q \vee \bar{q}])$ and $(\overline{\mathrm{p}} \vee[\mathrm{q} \wedge \overline{\mathrm{q}}])$, respectively, then $\left(\mathrm{p}^{*} \mathrm{p}\right)$ and $(\mathrm{p} \ \mathrm{p})$ are really just $\approx \mathrm{p}$ and $\sim \mathrm{p}$.

5 From the practical point of view there is much value in having an ordinary language surface grammar that allows us to negate a proposition by a simple syntactic device such as affixing $\sim$ (or some such equivalent) to the proposition. For in the application of the ontological grammar of (our variant of) logical atomism as a theory of meaning we decode a surface grammar negative proposition by computing the dual of the proposition negated. Such computation, like most decoding of ordinary language, would be impractical to execute in the surface grammar of ordinary language itself.

6 In terms of truth-tables: among truth-tables containing the elementary propositions occurring in $\varphi, \varphi$ is true in row $\mathrm{R}$ of one of the truth-tables iff $\varphi$ is true in any row $\mathrm{R}^{\prime}$ of any other such truth-table, where $\mathrm{R}$ and $\mathrm{R}^{\prime}$ agree in the truth-values assigned to the elementary propositions occurring in $\varphi$.

7 In terms of truth-tables: a proposition is true in every row of a truth-table iff it is true in every row of every truth-table containing its component elementary propositions.

8 Kripke's semantics in truth-tabular. The equivalence between the two is easily seen by noting that the elementary propositions of a logical space are simply the elementary propositions of a truth-table; and the sets of worlds of a logical space are simply the sets of rows of the corresponding truth-table.

9 Suppose $\varphi$ is universally valid and $\mathscr{H}$ is a logical space of which $\varphi$ is a proposition. Then, by assumption, $\varphi$ is $W(\mathscr{Y})$-valid (in $\mathscr{P})$. But obviously $W(\mathscr{P})$ validity (in $\mathscr{P}^{\mathcal{P}}$ ) amounts to validity (in $\mathscr{Y}$ ) simpliciter; and therefore, by theorem $1, \varphi$ is logically true.

10 It should not be thought that the notion of $\mathrm{K}$-validity as characterized here provides in any way a philosophically satisfying semantic analysis of logical necessity as a real material "property" of propositions. Certainly, within the context of atomism and the presumed independence of non-complementary atomic situations, the notion of K-validity is philosophically incoherent. Apparently, its philosophical coherence, if any, depends upon a type of metaphysical framework which is radically different from logical atomism. And in that case it probably will not even represent logical necessity but some other modality the material content of which is essential to the metaphysical scheme in question. But in any case, it is our general claim that without even an informal specification of a presumed philosophical framework, algebraic or model-theoretic semantical analyses are devoid of philosophical significance. It is only relative to a philosophical framework that philosophical significance or coherence can be accorded such analyses. 
11 Without $\square$ and $\diamond$ in the ontological grammar, quantifiers for propositional variables are eliminable and therefore ineffective as marking an ontological commitment. But with $\square$ and $\diamond$ present, quantifiers for propositional variables are no longer eliminable. Thus the comprehension principle we have in mind is not of the form $\exists \mathrm{p}(\mathrm{p} \leftrightarrow \varphi)$, where $\varphi$ is an arbitrary proposition in which the variable $p$ does not occur. This formula is trivally provable since $[(\mathrm{q} \leftrightarrow \varphi) \vee(\sim \mathrm{q} \leftrightarrow \varphi)], \exists \mathrm{p}(\mathrm{p} \leftrightarrow \mathrm{q})$ and $\exists \mathrm{p}(\mathrm{p} \leftrightarrow \sim \mathrm{q})$ are all readily provable. The comprehension principle in question is rather of the form $\exists p$ $\square(\mathrm{p} \leftrightarrow \varphi)$, where $\varphi$, p are as described above.

12 A similar commitment to material properties and relations of objects but not to formal or internal properties and relations of objects requires a substitution rule that distinguishes primitive predicate constants representing real (material) nexuses from defined predicates or open formulas representing only formal or internal properties and relations of objects. We should note here, incidentally, that the nominalist will have to distinguish between syncategorematic signs that are mere "punctuation-marks" and others like predicates and elementary propositional signs that somehow have ontological significance that is more than that of mere "punctuation-marks." Have we here entered the realm of subsistent entities? How otherwise might nominalism and logical atomism have a non-empty intersection?

13 The qualification that neither q nor its complement occurs in $\varphi$ is required since, for example, from the substitution of $q$ for $p$ in the logical truth $\diamond(p \wedge \sim q)$, where $p, q$ are distinct and non-complementary, we have the logically false proposition $\diamond$ (q $\wedge$ $\sim$ q). The qualification is appropriate to our criterion for nominalistic commitment since, by assumption, different elementary propositional signs represent different atomic situations.

14 Although no prior published account has bcen given of it, Kaplan formulated S13 (with negation included as a primitive connective) in the late 1950's. The semantics was the truth-tabular variant and the completeness theorem was in terms of certain normal forms. No special association was made then that I know of between $\mathrm{S} 13$ and logical atomism.

\section{References}

[ACT] Kripke, S., "A Completeness Theorem in Modal Logic," Journal of Symbolic Logic, vol. 24 (1959): 1-14.

[NAS] Sellars, W., "Naming and Saying," Philosophy of Science, vol. 29 (1962): 7-26.

[TR] Wittgenstein, L., Tractatus Logico-Philosophicus, trs. by D.F. Pears and B.F. McGuiness (London: Routledge and Kegan Paul, 1961). 


\section{Logical Atomism, Nominalism, and Modal Logic}

Logical atomism, through its theory of logical form, provides one of the most coherent formal ontologies in the history of philosophy. It is a coherence which, whether we agree with the ontology or not, renders the framework important and useful as a paradigm by which to compare and better evaluate the coherence of alternative systems based upon alternative theories of logical form and especially alternative theories of predication.

As the basis of a formal ontology, logical atomism, aside from the differences between its realist and nominalist variants, specifies not only a 'deep structure' ontological grammar within which all analysis must ultimately be resolved, but determines as well a logistic for that grammar. Both together constitute the formal ontology and serve to indicate how logical atomism views the fundamental structure of reality. Thus, for example, the grammar serves to indicate the formal as well as the material categories of being acknowledged by the ontology, while the logistic, by regulating the proper 'logico-syntactical employment' ([TR], 3.327) of the expressions of that grammar serves to indicate not only the logical 'scaffolding of the world' ([TR], 6.124) but supplements the grammar in its representation of the ontological structure of reality.

The distinction between logical scaffolding and ontological structure is 
fundamental to atomism and pertains to a distinction between material and formal content that grammar alone is insufficient to represent. It is a distinction that any proposed formalization of logical atomism must account for (through the Doctrine of Showing) in order to be an adequate formal representative of that ontology. It is a distinction, however, or so it will be argued here, that cannot be made without the introduction of modal operators for logical necessity and possibility.

The argument for this last claim was already given in chapter 6 , but it was there restricted to the level of logical analysis dealing solely with propositional connectives. Relative to that level it was shown that with the proper 'logico-syntactical employment' of the grammar, the internal 'properties' of propositions represented by modal operators for logical necessity and possibility need be no more material in content than are the internal 'relations' represented by the conjunction and disjunction operators, and that, accordingly, the introduction of these modal operators need not result in any form of essentialism according to which some material, as opposed to merely formal, content is represented as either logically necessary or logically impossible. ${ }^{1}$ In particular, it was shown in chapter 6 that we can characterize an appropriate logistic for logical atomism, at least for the level of analysis dealing solely with propositional connectives and which includes modal operators for logical necessity and possibility, so that a propositional sign (Satzzeichen) is logically true just in case the proposition expressed by that sign is logically necessary. In other words, in a logistic where the modal operators are given their proper logico-syntactical employment, there is a complete concurrence between the formal and the internal 'properties' of propositions as these are represented by the invariant semantical properties of their signs on the one hand and by the occurrence of modal operators within those signs on the other. Accordingly, so long as the modal operators are an integral part of the 'deep structure' ontological grammar for logical atomism, we do not need, at least for the sentential level of analysis, to resort to a semantic ascent where we must station ourselves outside the world in order for a proposition to show (zeigen) its formal or internal 'properties' and 'relations'. And if that is so, then we similarly do not need to resort to a Tractarian ladder that rests on alien ontological grounds, and which accordingly we could not then throw away, in order 
for the formal ontology of logical atomism to show by means available within itself the distinction between material content as real ontological structure and formal content as logical scaffolding. ${ }^{2}$

In what follows we shall be concerned with the problematic extension of these results to the level of analysis involving quantifiers for objects as concrete particulars along with some means for expressing their selfidentity and mutual difference. On this level, logical atomism's theory of predication enters our considerations in a fundamental way. For according to that theory, only elementary predications represent or 'picture' a structure with material content, and that content is in all cases external to the constituents of the structure. Such a structure is an atomic situation (Sachlage) and the externality of its content to its constituents consists in both it and its complement being logically possible. The difficulty here is that since objects are quantified over, they are part of the world and therefore contribute to the ontological content of the world (cf. [TR] 5.5561); and in that regard their self-identity and mutual difference or nonidentity, and thereby their total number, would prima facie seem to involve material content. Yet, in atomism, an object's self-identity or nonidentity with any other object is not an external condition of that object, ${ }^{3}$ and, as a consequence of the dependence of logical space on reality, it is logically impossible for the totality of objects, no less the number of that totality, to differ from world to world. In other words, in logical atomism, if not in other ontologies, identity and difference, as well as objectual quantification, are formal and not material aspects of reality. Here already we begin to see the paradigmatic role of logical atomism, for in most other systems identity and difference, as well as objectual quantification, are also said to be formal in content, though propositions regarding that content are not also said to be either logically necessary or logically impossible.

Because our considerations will be restricted to quantifying over objects as concrete particulars and not, for example, over material properties and relations as well, the variant of logical atomism we shall discuss here is nominalistic. Several realist alternatives are sketched in order to highlight the significant theses and/or difficulties of nominalism, though it should be noted that not all forms of nominalism need agree with the special ontological theses of nominalist logical atomism.

Finally, it should also be noted that our concern in this chapter is with an adequate formal representation of the ontology of logical atomism 
and not with its theory of thought, meaning, or philosophy of language. We wish to leave open how these might or must be developed with respect to the system constructed here, especially with regard to how they might or must pertain to the question of its logistic completeness.

\section{Negative Facts and Complementary Nexuses}

According to the variant of atomism we shall consider here, reality consists in the existence and non-existence of atomic situations ([TR], 2.06). Although that sounds like Tractarian orthodoxy, what we intend is not. For, along with Russell, we understand the nonexistence of an atomic situation to be a material 'property' or condition of that situation, viz., its negative facticity. In this regard, the being of an atomic situation is its being-the-case-or-not-the-case, that is, its existence-or-nonexistence. Naturally existence and nonexistence are presumed to be ontologically exclusive and exhaustive material conditions of atomic situations, in which case the being of an atomic situation as constituted by such a disjunction, is a formal 'property' of that situation, since it is a 'property' which the situation has in every possible world. Possible-existence is also a formal 'property' of atomic situations, but it is inadequate as a ground for the being of an atomic situation. Atomic situations have being not because they exist in possible worlds, but rather possible worlds are possible precisely because they are resolvable into atomic situations that are already materially grounded in their being-the-case-or-not-the-case. In other words, possible worlds are grounded in reality, the existence and nonexistence of atomic situations, and not otherwise.

By the way of contrast, note that this distinction between being and existence does not apply to objects, the constituents of atomic situations. The being of an object is its existence, for an object exists not only in the one world that is reality but in every logically possible world based upon reality. In this sense the existence of an object is not an external material property or condition of that object; and therefore, according to the atomistic theory of predication, the existence of an object is not to be represented by an elementary predication of the formal ontology.

Atomic situations, on the other hand, are the direct correspondents of elementary predications, and on the assumption that there is material content in those that do not exist as well as in those that do, both, therefore, 
are to be represented by elementary predications. Accordingly, since existence and nonexistence are exhaustive as well as exclusive conditions, the negation of an elementary predication must itself be represented by an elementary predication. ${ }^{4}$ Ontologically, this means that corresponding to each atomic situation there is another atomic situation that is its complement and of which it is the complement.

This complementation between atomic situations is not itself ultimate, however, but is grounded in a deeper complementation between nexuses or modes of configuration. Every atomic situation is a configuration of objects. The mode of that configuration is the nexus of the situation. And for each such nexus there is another nexus which is its complement and of which it is the complement. It is this complementation between nexuses that grounds the materiality of negative facts.

A fuller understanding of nexuses and their complementation, however, confronts us with atomism's version of the realist-nominalist controversy. For it is just in what each takes the nexus of an atomic situation to be that atomistic realists and nominalists disagree. (Cf. [NAS].) For example, according to one variant of realist logical atomism, material properties and relations are a kind of object and occur and are quantified over as such in atomic situations. The nexus of a situation is then an exemplification of a material property or relation by the other object or objects of the configuration. As a nexus, exemplification is not itself a material relation but a syncategorematic 'tie' between objects, and in that regard Bradley's infinite regress argument is not a problem here. ${ }^{5}$ Given complementation as the presumed ontological ground for negative facts, this variant of realist logical atomism requires that there be two exemplification nexuses, each the complement of the other, as the modes of all configurations having the same degree of complexity of constituents. ${ }^{6}$

For the nominalist, however, material properties and relations are not themselves constituents of atomic situations but are rather the very nexuses of these situations. Moreover, as nexuses, material properties and relations, despite their material content, are said by the nominalist not to be part of the world and therefore cannot be quantified over as such. In other words, a material property or relation is for the nominalist what exemplification is for the realist: a syncategorematic tie between objects. Only, for the nominalist the tie, as a material property or relation, has ma- 
terial as well as formal content, whereas for the realist the tie has only formal content.

The oddity of a syncategorematic tie with material content seems hardly to have been recognized or appreciated in the literature, no less clarified by nominalists. The combination is of course of dubious coherence since the distinction between formal and material content is normally thought to be absolute in logical atomism. Nevertheless, it is an oddity which nominalist logical atomism is committed to, since in that framework nexuses as material properties and relations must have material content while yet not being quantified over as part of the world they are accorded the status of being only formal or syncategorematic ties. What in this framework the nominalist is committed to recognizing as material content in such a tie the realist in his framework 'objectifies' and construes as an additional constituent of the situation, calling the remaining purely formal content exemplification. For the nominalist, however, only concrete particulars are objects and these constitute the unalterable material substance of the world. As nexuses, material properties and relations are not part of the material substance of the world, and for nominalism they can therefore only be accorded a formal or syncategorematic status.

In any case, since nexuses for the nominalist are material properties and relations, complementation must then consist in there being a unique, distinct complement for each material property or relation. Such a complement, it should be noted, is itself a simple nexus of atomic situations and is not to be understood as a complex material property or relation. For, as a fundamental thesis of logical atomism, there can be no nexus which is a complex of one or more other nexuses. That is, every nexus is the nexus only of an atomic situation; and other than its complement, no nexus can (onto)logically exclude or contain any other nexus: every atomic situation is (onto)logically independent of every other noncomplementary atomic situation. ${ }^{7}$ Accordingly, as nexuses of atomic situations, there can be no complex material properties or relations. ${ }^{8}$

There is a variant of realist logical atomism which agrees with nominalism in its construal of material properties and relations, along with their complements, as the nexuses of atomic situations. As with nominalism, exemplification is not a syncategorematic tie here, and all and only concrete particulars are objects. But in opposition to nominalism and in ap- 
parent agreement with realism, material properties and relations have a categorial and not a syncategorematic or formal mode of being. That is, material properties and relations are for this variant of realism part of the world and as such are indicated by quantifiable predicate or nexus variables (of appropriate degree). Thus while agreeing with nominalism in its construal of material properties and relations as nexuses, it disagrees with nominalism in the latter's view of nexuses as syncategorematic ties between objects. Indeed, for this variant of realism there is no syncategorematic tie at all between the objects in an atomic situation, only a real material property or relation instead. Of course, although they are part of the world, quantification over nexuses as the (onto)logical structures of atomic situations is ontologically incomparable with quantification over objects, the constituents of these situations. And in no way is this form of quantification to be construed as a restricted form of quantification over individuals (objects), despite its superficial appearance as such in the surface grammar of ordinary language. Similarly, in the application of the formal ontology for such a variant of realism, the introduction and association of predicate constants, or of linguistic nexuses if the scheme of notation is 'perspicuous', with the nexuses of atomic situations is semantically, as well as ontologically, incomparable with the introduction and association of names with objects. Whether such an association of predicates with nexuses be called denotation (Bedeutung), in the manner of Frege, or pictoral form (Form der Abbildung), in the manner of Wittgenstein, is inconsequential so long as it is not confused with the completely different association of names with objects. ${ }^{9}$ It is our view, and Sellars's as we understand his exposition in [NAS], that the atomism of the Tractatus is realist in this sense and not nominalist as generally seems to be thought.

In contrast to this variant of realism with its two fundamental but incomparable categorial modes of being, nominalism is a form of categorial monism according to which the only categorial mode of being is that of concrete particulars. In the context of a formal ontology where syntactic categories are distinguished by equivalence classes of mutually substitutable expressions salva validitate, nominalism takes the form of an injunction against allowing distinctive styles of quantifiable variables for any syntactic categories besides that of the individual or nominal expressions. Free individual or nominal variables are then interpreted as representing 
the mode of being of individual objects or concrete particulars while existentially binding them expresses the categorial existence of their values. In logical atomism, where the being of an object is its (categorial) existence, the same objects that are values of free variables are of course therefore also values of bound variables. It is not clear whether this last must be true for all forms of nominalism. ${ }^{10}$

Note in this last regard that if material properties and relations are a real part of the world and were accorded a categorial mode of being, as in either variant of realism, then, according at least to logical atomism, there would be a divergence between their categorial being and their existence, or rather their existence in the sense of actually being exemplified, as in the first variant of realism, or, as in the second variant, being the nexus of an existing atomic situation. For according to logical atomism, every atomic situation can be the case or not the case regardless of what other non-complementary atomic situations are the case, and therefore every atomic situation containing a given material property or relation as a component, whether as an object or as the nexus of the situation, might well not be the case; that is, there will always be a possible world, perhaps the real world itself, in which the given material property or relation will not exist in the above sense, while yet since it does exist (in the same sense) in other possible worlds (of the same logical space) it must be accorded categorial being (within that logical space) and be so indicated by the bound predicate or nexus variables of the appropriate degree. Perhaps it is this divergence between categorial being and existence, i.e., this divergence of existence even within the range of quantifiers, which is the basis of the nominalist's injunction against quantifying over nexuses. If so, it is of dubious philosophical merit since the divergence is fundamental to any fact ontology in which negative, but not possible, facts have material content. ${ }^{11}$

On the other hand, the traditional nominalist argument against 'universals' on the ground of their simultaneous multiple existence (in the above sense) in different places seems inopportune, if not irrelevant, prior to an atomistic account of space and time-an account which when it is finally given may cast doubt more on the nature of objects than on that of nexuses. Besides, in atomism, there is an appropriate sense in which objects as well as nexuses are 'universals': both are 'unsaturated' or incomplete, though each is so with respect to the other in a completely complemen- 
tary manner, as 'lock and key', so that each completes the other in an atomic situation. ${ }^{12}$ But which is lock and which is key? Both have and fill 'holes' which are complementary to the other. Both can play the One while the other plays the Many. Neither really corresponds very well to the particulars and universals of traditional nominalism.

We might point out here that for our second variant of realism, though perhaps not also for nominalism, there is one criterion which might distinguish objects as concrete particulars from nexuses as abstract universals. And that is the possible cardinalities of each in the world. As constitutive of the material substance in the world, objects cannot be infinitesimal point centers, and in that regard (assuming Cantor's continuum hypothesis) their total number cannot exceed $\aleph_{0}$ in cardinality. On the other hand, as the basis of the logical and ontological structure of the world, especially its spatio-temporal structure, nexuses might well exceed $\aleph_{0}$ in cardinality, notwithstanding their material content. This difference together with the metaphysical thesis that a type of entity is abstract if the total number of entities of that type in the world $\operatorname{can}^{13}$ exceed $\aleph_{0}$ and concrete if that number cannot exceed but can be less than $\aleph_{0}$ suffices to ground the claim that objects are concrete particulars while nexuses are abstract universals. ${ }^{14}$ Nominalism, however, or so it would seem, cannot resort to this criterion since nexuses are supposedly not part of the world and cannot therefore be quantified over for the determination or representation of their number. Instead, as metalinguistically distinguishable syncategorematic ties of a formal ontology, nexuses would seem to be limited in number, as would all the syncategorematic or formal elements of the system, so as not to exceed $\aleph_{0}$. For this reason in what follows we shall assume that both the number of objects in the world and the number of nexuses as metalinguistically distinguishable syncategorematic ties between these objects do not exceed $\aleph_{0}$.

\section{Nominalism's View of Logical Space}

Reality, we said, is the existence and nonexistence of atomic situations ([TR] 2.06). The world, on the other hand, is the sum total of reality ([TR] 2.063). And an alternative possible world is simply an alternative combination of all the same atomic situations constitutive of the world. That is, there are no possible facts that are not already facts, positive or 
negative, of the one world that is the sum total of reality; and a possible world is an alternative combination of these same atomic facts in the sense that any or all of the negative facts of the world may be thought of as existing (in that alternative world) while their complements, the existing situations in reality, are thought of as nonexisting. Here in logical atomism with negative facts, we find what is perhaps the only coherent, ontological account of the nature of a logically possible world. For this reason, if none other, it is to logical atomism we might well turn for our paradigm modal logic. All others might then be better understood and evaluated for their own coherence in terms of how the framework they are based upon differs from logical atomism.

If we now ignore the particular material or 'existential' status of atomic situations, i.e., their status as constitutive of the world rather than of an alternative possible world, and consider them merely in their being and as constitutive of all possible worlds, then, instead of reality, their sum total is logical space. This difference between reality and logical space is fundamental to our distinction between ontological structure and logical scaffolding, i.e., between formal and material content. For while logical space is ontologically based upon reality, the logical structure of reality is based upon logical space. Language, or rather that philosophically relevant underlying structure of language that we have called formal ontology, is to represent and duplicate within itself the mediation between the two.

Let us then turn to this mediation between reality and logical space and its representation or replication within the formal ontology of nominalist logical atomism. Logical space, we said, consists of all the atomic situations in reality, those that are not the case as well as those that are. And each atomic situation, as noted, is a configuration of objects, the mode of which is a nexus, i.e., a material property or relation, which in nominalism is a syncategorematic tie between the constituents of the situation. Language, or rather its underlying formal ontology, cannot present us with these properties and relations in their full materiality but it can, if it is 'perspicuous', present us with their linguistic counterparts that share the same (onto)logical structure. And for the replication of the mediation between reality and logical space, preservation of such structures is all that is essential.

Perspicuous linguistic nexuses, on the other hand, are not essential for an adequate symbolic replication of the structures of nexuses. There are 
other, and for our purposes more appropriate, symbolic means for representing these structures. For example, given their proper logicosyntactic employment, predicate constants can indirectly be construed as linguistic nexuses, even though when employed they are a constituent and not the nexus of an elementary propositional form. This approach allows us to uniformly utilize linear arrangements for all expressions of whatever complexity. Naturally, these linear arrangements do not represent linear nexuses per se, and the proper logicosyntactic employment of predicate constants occurring in them is not that of names or expressions for individuals.

In regard to the proper employment of predicate constants, let us note that the structure of a nexus is adequately represented by three syntactic factors, two grammatical and one logistic. The two grammatical factors are its degree and order. The first determines the number of different objects with which it can unite to form a configuration, and the second is the order in which these objects are to be associated with their linguistic counterparts in an elementary proposition representing such a configuration. However, since in the standard linear notation, which we shall adopt here, the order factor is represented in the linear arrangements of expressions for objects, we shall hereafter speak only of the degree of a predicate constant.

The logistic factor pertaining to predicate constants as syncategorematic nexuses is that no two of them can be ties in the same way between all the same objects in all the same possible worlds (of a logical space). ${ }^{15}$ For whether the nexuses represented by two such constants are configured in the same way with all the same objects may well be a non-atomic fact in the world; but because of the independence of non-complementary atomic situations, that these nexuses should be configured in the same way with all the same objects in every possible world could be no matter of fact but only of an internal necessity showing their 'identity'. Accordingly, were there to be different predicate constants that were necessarily equivalent, they would have to be understood to represent the same and not different nexuses.

Nominalism, of course, despite the convenience of informal discourse, finds no more significance in speaking of nexuses as the same or different than it does in granting them categorical existence via quantifiers and speaking of all or some of them. Instead, nominalism replaces talk of nexuses by talk of their linguistic counterparts, i.e., predicate constants in the 
present notation. And in that regard there can be no significance in speaking of different predicate constants as representing the same nexuses. Accordingly, as part of their logistic role in nominalist logical atomism, different predicate constants of the same degree cannot be necessarily equivalent ${ }^{16}$ (Cf. [TR] 5.451).

There is one difficulty about the nominalist's view of predicate constants that should be commented on here that pertains to their rather unique role as syncategorematic signs with material content. We have already pointed out that though in nominalism a nexus is only a syncategorematic and not a categorial tie between objects, it is nevertheless not without material content; that is, a nexus is after all a material property or relation, even if as such, according to nominalism, it is not quantified over as part of the world. In other words, though nexuses are logical structures, they are not part of the logical scaffolding of the world in quite the way the logical operations represented by propositional connectives are, or the way other predicative logical structures represented by valid open propositional forms are. Indeed, as the ontological basis for that scaffolding, nexuses are ontological structures with material content, and predicate constants in so far as they are to represent or replace these structures are therefore to play the role of syncategorematic signs with material content. The nominalist's difficulty then is how he can coherently reconcile and express both the formal and material role of predicate constants.

The answer, in part, lies in the Doctrine of Showing according to which "what can be shown cannot be said" ([TR] 4.1212) though it may appear to be 'said' in the surface grammar of ordinary language and is in that regard a pseudo-proposition (Scheinsatz). We note, however, that relative to the propositional forms of a formal ontology, what can be shown might be shown by the ontological grammar alone or it might require the logistic behavior of that grammar as well. For example, that no nexus is an object is an 'ontological fact' that, like all such 'facts', cannot be said in the formal ontology of atomism; it can, however, be shown by the ontological grammar of that ontology. ${ }^{17}$ Similarly, that a nexus in the formal ontology of nominalist logical atomism has a formal and not a categorial being shows itself in part in the grammatical fact that ( $n$-place) predicate constants are syncategorematic signs, i.e., the fact that these constants are not substituents of variables of any syntactic type, and especially not of a type characteristic of ( $n$-place) predicate expressions in general. This al- 
ready involves logistic to some extent, but it is also reflected in there being no bindable predicate variables in the formalism. In any case, that a nexus is a material and not a purely formal property or relation is an ontological fact that requires logistic as well as grammatical distinctions for its expression.

In the realist framework that takes material properties and relations to be nexuses, essentially the same pseudo-proposition is expressed in the form of certain logistic constraints placed upon the comprehension principle for nexuses, or, equivalently, upon the substitution of propositional forms in the universal instantiation law for nexuses. These constraints are to the effect that only propositional forms that represent an $n$-ary nexus can be properly substitued for a generalized $n$-place predicate or nexus variable, or, equivalently, that only these forms show themselves to 'comprehend' an $n$-ary nexus. ${ }^{18}$ Thus, a propositional form that represents a purely formal property or relation, e.g., being self-identical, or being disjointly configured by a certain monadic nexus or its complement, etc., will not satisfy these constraints, though a predicate constant in an elementary propositional form will. In this way the realist is able to distinguish the (categorial) material content of a predicate constant from the purely formal content of other predicative expressions (open propositional forms).

Nominalism, of course, cannot express its version of the pseudoproposition in quite this way, but it can and does parallel the realist's distinctions and constraints in its variant of the universal instantiation law for nexuses, viz., the rule of substitution of open propositional forms for elementary predicational forms. ${ }^{19}$ And that in a way is rather appropriate, for where the realist quantifies over nexuses as part of the world the nominalist quantifies over metalinguistically distinguishable predicate constants as syncategorematic signs. Of course, in the end, every such metalinguistic ascent, unless it be from alien ontological grounds, amounts to climbing a Tractarian ladder where what is 'said' on such a ladder must, once the ladder is thrown away, be shown in the propositional forms of the formal ontology if it cannot already be directly said by those propositional forms themselves. And that a predicate constant has material significance is just such a pseudo-proposition and is shown in the proper substitutional role of elementary predicational forms containing predicate constants. But then, since the 
substitutional role of elementary predicational forms in standard predicate logic without modal operators for logical necessity and possibility is no different than that for complex propositional forms, standard predicate logic without these modal operators is therefore inadequate as a formal representative of nominalist logical atomism. Significantly, and very much to the point of the present paper, standard predicate logic supplemented with these modal operators along with their proper logicosyntactic employment does suffice, on the other hand, to make the relevant substitutional distinctions.

There is one remnant of the nominalist's difficulty with predicate constants left us, however. And that pertains to the pseudo-proposition that nexuses are syncategorematic and not categorial ties between objects. Merely refusing to include quantifiable predicate variables in the grammar is not enough to express this pseudo-proposition in the context at least of logical atomism. For what is also required in this context is that each and every predicate constant is a fixed logical constant of the nominalist's theory of logical form. That is, even though the substitution rule will show predicate constants to have material content not shared by complex predicational forms, these constants are nevertheless not to be construed as applied descriptive constants. The nominalist, in other words, cannot coherently construe the introduction of predicate constants as an application of his theory of logical form, and especially not as an application of his theory of predication. "What belongs to its application logic cannot anticipate. .. . Therefore, logic and its application must not overlap" ([TR] 5.557). Consequently, for the nominalist predicate constants are, as it were, locked into his theory of predication and cannot be added to or deleted from that theory without essentially altering its structure. And therefore, in so far as a theory of logical form is not an empirical theory but precedes all such theories, whether or not there is a 27termed syncategorematic nexus is for the nominalist, though apparently not for the realist, to be settled a priori after all, contra Tractarian orthodoxy (Cf. [TR], 5.5541).

The nominalist's typical response to this difficulty is to resort to dummy or schematic predicate letters of arbitrary degree in the characterization of his theory of logical form. He might even adopt some of the grammatical terminology of the realist and refer to such dummy predicate letters as (free) variables, though of course quantifiers are not al- 
lowed to bind them. Then, where the realist would assert the categorial existence of an $n$-ary nexus by means of an existential quantifier, the nominalist merely introduces an $n$-place predicate constant into the object language, thereby, so he claims, resorting to his usual metalinguistic ascent in order to parallel the realist's categorial assertion.

But can what appears to be 'said' metalinguistically in this case really be shown in the propositional forms of the nominalist's system? That is, is not the nominalist ascending a ladder in this case that really rests on alien ontological grounds? For is this introduction of a predicate constant really parallel to the realist's categorial claim? Is it not parallel instead to what for the realist is an application of his theory of logical form? Indeed, is it not an application of the nominalist's presumed theory of logical form supposedly characterized in terms of dummy predicate letters? And in that case does it not purport to be an application of his theory of predication that really requires the supposed application for its basis? For to suppose that dummy predicate letters suffice for this purpose, while denying any and all significance to quantifying over them, amounts to an assumption that logical space can be characterized independently of reality, a haunting, familiar theme, but quite alien to logical atomism. "Logic pervades the world: the limits of the world are also its limits" (5.61).

And this situation is even more pronounced when the nominalist attempts to parallel the realist's assertion of categorial nonexistence for nexuses of a certain degree. For in that case the nominalist must presuppose the significance of a predication pattern or logical form characterized in terms of dummy predicate letters that, according to his own theory of predication, has nobasis in reality. ${ }^{20}$

In the end then, given reality and the grounding of logical space upon reality, the logical forms nominalism can meaningfully characterize are those based upon the elementary propositional forms containing the predicate constants it must presuppose to represent all and no more than the nexuses configuring the atomic situations of logical space, i.e., the atomic situations that are or are not the case in reality.

And yet, were reality to contain more nexuses than it does, especially nexuses of a degree of complexity not already in reality, then the logical forms available to nominalism would also be thereby augmented. But then even were this so, because of the fundamental thesis of logical atomism, viz., the mutual independence of noncomplementary atomic sit- 
uations, the propositions that according to nominalism now show the logical structure of the world would not then show a different structure as a result of these additional forms. Unfortunately, it is precisely the presumed sense of this apparent contrary-to-fact conditional which nominalism seems to have cut off from itself. For what it represents is not a possibility that can be realized in logical space, since the latter is based upon reality with the nexuses it does contain. Indeed, it is in a way a kind of 'ontological contingency' that is not reducible to logical possibility.

Shortly, we shall confront and discuss a similar sort of ontological contingency, viz., that regarding the total number of objects. And for nominalist logical atomism, it is this supposed 'contingency', and not that regarding the number and degree of nexuses, which disrupts the proper concurrence of logical truth as indicative of a formal 'property' of propositions with logical necessity as an internal 'property' of those same propositions. In our examination below of this supposed 'contingency', we shall tentatively allow the problematic introduction of 'different realities' with differing total numbers of objects. And to the extent that differing realities are allowed at all, we shall allow them to differ in their total number and degree of nexuses as well. The nominalist's 'counterfactual' claim will then come to this: the 'contingency' of the total number and degree of nexuses in reality does not go beyond the questionable 'contingency' of the total number of objects. That is, if a proposition that is expressed by the nominalist's ontological grammar as based upon the nexuses in reality is logically necessary independently of the number of objects in the world, then that proposition would still be logically necessary even were reality to contain more nexuses than it does. And this will be so precisely because of the atomistic nature of reality, viz., the mutual independence of noncomplementary atomic situations.

\section{An Abstract Semantics for Nominalist Logical Atomism}

In turning now to a formal or abstract semantical presentation of logical atomism, let us tentatively allow the sort of ontological contingencies in question. We do so by climbing a ladder where it appears we can 'say' that there are 'different realities' with differing total numbers of objects and syncategorematic nexuses of differing degree. In the end of course, if what is 'said' here cannot be either said or shown in the propositional 
forms of nominalist logical atomism, then we must admit ourselves to be speaking from a ladder that rests on grounds alien to that ontology.

In what follows we assume the nexuses of each reality to be identified with a set of predicate constants each of a specified degree. No limitation is presumed in the number and variety of degrees of predicate constants so associated with a given reality other than that the degrees must be finite $^{21}$ and that the number of such constants cannot exceed $\aleph_{0}$. In this way the purposes that dummy predicate letters have been thought to serve are served here instead by predicate constants along with the problematic introduction of differing realities. The approach here is preferable, at least in the context of atomism, since the dubious ontological assumption upon which it is based is made quite explicit.

We shall in general bypass speaking of different realities, however, since our present concern is with logical and ontological structure and not with which atomic situations are actually the case or not the case in a given reality. We shall proceed then directly to the logical spaces based upon these realities. Naturally, because each is based upon its respective reality, it should not be thought that speaking of different logical spaces is somehow less problematic.

Abstractly, a logical space can be 'individuated' as follows. We assume that corresponding effectively to each predicate constant of a given degree there is another predicate constant of the same degree and such that each is the complement of the other. Where $\pi$ is an $n$-place predicate constant, for an arbitrary positive integer $n$, we take $\bar{\pi}$ to be the complement of $\pi$, and, accordingly, $\overline{\bar{\pi}}=\pi$. It is now of course unnecessary to specify all the predicate constants of a logical space, since a set of noncomplementary predicate constants will suffice. Such a set is one of two factors in the individuation of a logical space. The other factor is the totality of objects that are constituents of the atomic situations constituting that logical space. It is noteworthy and relevant that these two factors determine our two sorts of ontological contingency.

Accordingly, we say that $S$ is an abstract logical space iff $S=\langle U, L\rangle$, for some set $L$ of noncomplementary predicate constants and some set $U$ of objects such that $U$ is empty iff $L$ is empty; in addition the cardinality of $U$ and $L$, respectively, is less than or equal to $\aleph_{0}$. We allow for the empty logical space (cf [TR], 2.013), but only when there are neither objects nor nexuses, since neither can 'be' without the other. 
Where $S=\langle U, L\rangle$ is an abstract logical space, we say that $s$ is an atomic situation in $S$ iff $s=\left\langle\pi, x_{1}, \ldots, x_{n}\right\rangle$, for some positive integer $n$, some $n$ place predicate constant $\pi$ such that either $\pi$ or $\bar{\pi}$ is in $L$, and some objects $x_{1}, \ldots, x_{n} \in U$. If $s=\left\langle\pi, x_{1}, \ldots, x_{n}\right\rangle$ we set $\bar{s}=\left\langle\bar{\pi}, x_{1}, \ldots, x_{n}\right\rangle$. Finally, we understand $\boldsymbol{U} \ell$ to be a possible world of $S$ iff $U$ is a set of atomic situations in $S$ and for each atomic situation $s$ in $S$ either $s \in \mathcal{U}$ or $\bar{s} \epsilon \mathcal{U}$, but not both.

We note that appropos of logical atomism the totality of objects configured in the atomic situations of one possible world of an abstract logical space is exactly the same totality as that of any other possible world of that space. This of course is the appropriate abstract representation of the atomistic thesis that the being or possible-existence of an object is its existence simpliciter. And this thesis, it should be emphasized, is not a posit of philosophical prejudice but a necessary consequence of the ontology. For whatever is a constituent of an atomic situation in reality is in every possible world of the one logical space based upon that reality a constituent either of the same atomic situation or of its complement, since by the principles of atomism it must be that either that situation or its complement is a fact of that other possible world. It follows from this that any pseudoproposition regarding the total number of objects in the world is, insofar as it is formulable in atomism at all, either logically necessary or logically impossible. Here we see quite clearly how the so-called contingency of this number is not reducible to the contingency of logical possibility.

In the way of noting another such consequence of the ontology, we should emphasize here that no proper subset of the set of all possible worlds of any abstract logical space can itself be the set of all possible worlds of either that or any other abstract logical space. That is, no such proper subset can be the range of significance of the metalinguistic quantification over all the possible worlds of any abstract logical space. The reason for this is that such a proper subset would exclude as logically possible either certain atomic situations whose complements happen to be the case in all the possible worlds of the subset or certain (possibly infinite) combinations of noncomplementary atomic situations the complements of which happen to be the case in all the possible worlds of the subset. In other words, as the range of significance for quantification over all the possible worlds of a logical space such a proper subset would represent either certain atomic situations as logically necessary or certain (possibly in- 
finite) combinations of noncomplementary atomic situations as logically impossible. And these are consequences totally alien to both the ontology and the theory of predication of logical atomism.

Yet, curiously, it is just such secondary or restricted interpretations of the notion of all possible worlds that is the basis of most of the contemporary semantics for modal logic. Indeed, as is clear from the conditions excluded, it is precisely because of such secondary or restricted interpretations that material content is imputed to the modal operators of these systems, which for that very reason are antithetical to the proper formal ontology of logical atomism. And, conversely, it is precisely because no such secondary or restricted interpretations are to be allowed in the semantics for logical atomism that the modal operators here have only formal and no material content after all.

\section{The (Onto)logical Grammar of Nominalist Logical Atomism}

Having abstractly characterized the global semantical structures for nominalist logical atomism, let us turn now to its ontological grammar and then to the abstract semantical association of the one with the other.

As primitive logical constants, we take, relative to a logical space, not only the predicate constants and their complements upon which the space is based but also the conjunction and disjunction signs, $\wedge$ and $\vee$, the (logical) necessity and possibility signs, $\square$ and $\diamond$, the (inclusive) universal and existential quantifiers, $\forall$ and $\exists$, and the identity and nonidentity (or difference) signs, $\equiv$ and $\not \equiv$. Note that the dual of each primitive logical constant is itself a primitive logical constant. This is required since negation is not a primitive logical operation, nor for that matter a logical operation at all. We realize that including the identity and nonidentity signs is contrary to the position of the Tractatus (cf. 5.533), but we shall take up the argument for doing so in a later section.

We assume the existence of an enumerably infinite set of individual variables (but note in passing that this assumption in atomism requires an infinite reality). We shall not deal with individual constants (as Tractarian names) here since they properly belong to the application of the formal ontology and not to the characterization of its grammar. Expressions in general are understood to be finite concatenations of the logical signs 
and variables, along with such auxiliary symbols as the left- and righthand parentheses. By an identity or non-identity formula we understand an expression of the form $\alpha \equiv \beta$ and $\alpha \equiv \beta$, respectively, where $\alpha$ and $\beta$ are individual variables. Abstractly, these expressions are formulable in every logical space.

Where $S=\langle U, L\rangle$ is an abstract logical space, we take the elementary propositional forms of $S$ to be all expressions of the form $\pi\left(\alpha_{1}, \ldots, \alpha_{n}\right)$, where $n$ is a positive integer, $\pi$ is an $n$-place predicate constant such that either $\pi$ or $\bar{\pi}$ is in $L$, and $\alpha_{1}, \ldots, \alpha_{n}$ are individual variables. The set of propositional forms of $S$, in symbols $F M(S)$, is the smallest set of expressions containing all the elementary propositional forms of $S$, each identity and nonidentity formula and such that $(\phi \wedge \psi),(\phi \vee \psi), \square \phi, \diamond \phi, \forall \alpha \phi$, $\exists \alpha \phi$ are in $F M(S)$ whenever $\phi, \psi$ are in $F M(S)$ and $\alpha$ is an individual variable. Note that though identity and nonidentity formulas are propositional forms of each logical space, they are not elementary propositional forms of any logical space, not even of the empty logical space, which contains no elementary propositional forms at all.

We assume the usual definition of bondage and freedom of (occurrences of) individual variables and understand a propositional sign (Satzzeichen) of the nominalist's formal ontology to be a propositional form in which no variable has a free occurrence. Also, although negation is not a logical operation in the present variant of atomism, it is convenient to introduce the negation sign by means of an abbreviational transformation: $\sim \alpha \equiv \beta=\alpha \equiv \equiv ; \sim \alpha \equiv \beta=\alpha \equiv \beta ; \sim \pi\left(\alpha_{1}, \ldots, \alpha_{n}\right)=\bar{\pi}\left(\alpha_{1}, \ldots\right.$ ,$\left.\alpha_{n}\right) ; \sim(\phi \wedge, \gamma)=(\sim \phi \bigvee \sim \psi) ; \sim(\phi \bigvee \psi)=(\sim \phi \wedge \sim \psi) ; \sim \square \phi=\diamond \sim \phi ; \sim \diamond \phi$ $=\square \sim \phi ; \sim \forall \alpha \phi=\exists \alpha \sim \phi ; \sim \exists \alpha \phi=\forall \alpha \sim \phi$. In this way when we refer to the negation of a propositional form we shall mean the dual of its contradual, i.e., the propositional form obtained from it by replacing each standard logical constant by its dual and each predicate constant by its complement.

Suppose $S=\langle U, L\rangle$ is an abstract logical space. Then a is an assignment to variables of values drawn from $U$ iff $a$ is a function with the set of individual variables as domain and such that for each variable $\alpha, \mathbf{a}(\alpha) \epsilon U$ if $U$ is not empty; otherwise $\mathbf{a}(\alpha)=0$. (Thus there is just one assignment for the empty logical space and that assigns 0 to each variable.) By a $\left(\begin{array}{c}\alpha \\ x\end{array}\right)$ we understand the assignment that is exactly like a except for its assigning $x$ to the variable $\alpha$; but if $\mathbf{a}$ is the assignment for the empty logical space, then 
$\mathrm{a}\left(\begin{array}{c}\alpha \\ x\end{array}\right)$ is just a itself. Where a is such an assignment in $S$ and $U$ is a possible world of $S$, then we recursively define satisfaction in $U$ by a (relative to $S$ ) as follows: if $\alpha, \beta$ are individual variables, a satifies $\alpha \equiv \beta$ in $U$ (relative to $S)$ iff $\mathrm{a}(\alpha)=\mathrm{a}(\beta)$; and a satisfies $\alpha \neq \equiv$ in $\mathcal{U}$ (relative to $S$ ) iff $\mathrm{a}(\alpha) \neq \mathrm{a}(\beta)$; if $n$ is a positive integer, $\alpha_{1}, \ldots, \alpha_{n}$ are individual variables, and $\pi$ is an $n$ place predicate constant such that either $\pi$ or $\bar{\pi}$ is in $L$, then a satisfies $\pi\left(\alpha_{1}, \ldots, \alpha_{n}\right)$ in $U$ (relative to $\left.S\right)$ iff $\left\langle\pi, \mathrm{a}\left(\alpha_{1}\right), \ldots, \mathrm{a}\left(\alpha_{,}\right)\right\rangle \epsilon \mathcal{U}$; if $\phi, \psi \epsilon$ $F M(S)$, then a satisfies $(\phi \wedge \psi)$ in $\mathcal{U}$ (relative to $S$ ) iff a satisfies $\phi$ in $\mathcal{U}$ (relative to $S$ ) and a satisfies $\psi$ in $\mathcal{U}$ (relative to $S$ ); a satisfies $(\phi \vee \psi)$ in $\mathcal{U}$ (relative to $S$ ) iff either a satisfies $\psi$ in $\mathcal{U}$ (relative to $S$ ) or a satisfies $\phi$ in $U$ (relative to $S$ ); a satisfies $\square \phi$ in $\mathcal{U}$ (relative to $S$ ) iff for each possible world $h$ of $S$, a satisfies $\phi$ in $h$ (relative to $S$ ); a satisfies $\diamond \phi$ in $U$ (relative to $S$ ) iff for some possible world $h$ of $S$, a satisfies $\phi$ in $h$ (relative to $S$ ); if $\alpha$ is an individual variable, than a satisfies $\forall \alpha \phi$ in $U$ (relative to $S$ ) iff for each $x \in U, \mathrm{a}\left(\begin{array}{l}\alpha \\ x\end{array}\right)$ satisfies $\phi$ in $U$ (relative to $S$ ); and a satisfies $\exists \alpha \phi$ in $\mathcal{U}$ (relative to $S$ ) iff for some $x \in U$, a $\left(\begin{array}{c}\alpha \\ x\end{array}\right)$ satisfies $\phi$ in $U$ (relative to $S$ ). Note that if $S$ is the empty logical space, then a satisfies every universally quantified, but no existentially quantified, propositional form of $S$.

If $S$ is an abstract logical space, $\mathcal{U}$ is a possible world of $S$ and $\phi$ is a propositional form of $S$, then $\phi$ is true in $U$ (relative to $S$ ) iff $\phi$ is satisfied in $U$ (relative to $S$ ) by every assignment in $S$. Also, $\phi$ is said to be valid in $S$ iff $\phi$ is true (relative to $S$ ) in every possible world of $S$.

Having tentatively allowed ourselves the problematic notion of different realities, and thereby that of different logical spaces, it is clear that logical truth, relative to the assumed coherence of such a notion, is to be identified with validity in every logical space. That is, a propositional form is logically true iff it is valid in every logical space of which it is a propositional form.

We might observe at this juncture that the possible worlds of an abstract logical space $S=\langle U, L\rangle$ correspond exactly to what in the formal semantics of standard predicate logic are the models suited to $L$, the set of non-complementary predicate constants upon which $S$ is based, and all the domains of which are one and the same set, viz., $U$, the set of objects of the space $S$. It is readily seen then that for modal free propositional forms, i.e., those in which neither $\square$ nor $\diamond$ occur, truth in a possible world of $S$ is equivalent to Tarski's notion of truth in the corresponding model, and accordingly, their validity in the logical space $S$ is equivalent 
to what in standard terminology is their validity in the domain $U$. Consequently, the logical truth of a modal free propositional form, as characterized here, coincides with its truth in every model suited to any set of predicate constants in terms of which it is formulable, which of course is its logical truth according to the standard model theoretic definition. These equivalences serve less to vindicate our present semantics for nominalist logical atomism than they do to indicate the suppressed or (what at least appears to be the) implicit philosophical background of the model theoretic semantics for standard predicate logic. It is perhaps of some historical significance to note that Carnap, the other founder besides Tarski of modern formal semantics, did have precisely this philosophical background in mind in his notion of a state description. Tarski's models and Carnap's state descriptions differ, we might note, precisely in the latter's essential use of individual constants as Tractarian names, a use which we exclude here since it belongs properly to the application of logic and not to its essential characterization.

It is curious, however, that the usual extensions of model theory to modal propositional forms depart rather radically from this implicit background of a logical space with its (onto)logically independent atomic situations. In general, as has already been mentioned, what these extensions introduce is precisely the sort of secondary or restricted interpretations of the notion of all the possible worlds of a logical space we discussed above, and in that regard, of course, they thereby impute material content to the modal operators for logical necessity and possibility. Occasionally, some of these extensions will also introduce similar secondary or restricted interpretations of the notion of all the objects existing in a possible world, though the background totality of objects as values of free individual variables remains the same throughout 'all' the possible worlds of the space. Naturally, according to these restricted interpretations for quantifiers the being or possible-existence of an object does not reduce to its existence as the value of a variable bound by a quantifier so interpreted, in which case the existence of an object is a material and not a formal or internal property of that object. And finally, though by no means the least among these points of departure, some of these extensions will introduce a primitive metalinguistic relation between the possible worlds of a logical space to the effect that some of the worlds are possible with respect to certain others but not necessarily to all the others of that same 
logical space. Such a departure suggests that possible worlds cannot be completely resolved into atomic situations after all but are instead possible 'world facts' of a holistic ontology.

Quite obviously, these developments of modal logic are completely antithetical to the principles of logical atomism, at least at the 'deep structure' level of its formal ontology. ${ }^{22}$ We do not dispute the philosophical significance of these developments here, but we do raise the question of the coherence of the sort of philosophical framework they presuppose. Naturally, the clarity of their abstract algebraic or set theoretical presentations is independent of the philosophical coherence of the alternative frameworks they presuppose. It seems appropriate, however, that the sort of coherence obtained in logical atomism might well serve as a paradigm by which to evaluate and better understand the coherence of these alternative frameworks.

Incidentally, aside from imputing material content to the modal operators, one of the typical consequences of these secondary or restricted interpretations of the notion of all the possible worlds of an abstract logical space is that the rule of substitution (barring clashes of bound variables) will hold regardless of the complexity of the propositional forms substituted for a predicate constant. In the semantics for logical truth, on the other hand, the modal operators have only formal content and the rule of substitution preserves logical truth when and only when applied to propositional forms logically equivalent to an elementary predicational form. ${ }^{23}$ And this is so precisely because quantification over the possible worlds of a logical space is not allowed a secondary or restricted interpretation.

Consider for example an arbitrary propositional form $\phi$ with respect to certain variables $\alpha_{1}, \ldots, \alpha_{n}$ as distinct argument indicators. Also, let $\pi$ be an $n$-place predicate constant. Then because of the independence of noncomplementary atomic situations and the fact that by all the possible worlds of an abstract logical space we do indeed mean all the worlds of that space, the propositional form

$$
\forall \alpha_{1} \ldots \forall \alpha_{n} \square\left[\pi\left(\alpha_{1}, \ldots, \alpha_{n}\right) \leftrightarrow \phi\right]
$$

will be either logically true (in which case either $\pi$ or $\bar{\pi}$ occurs in $\phi$ ) or logically false. If it is logically true, then $\phi$ represents, with respect to the variables $\alpha_{1}, \ldots, \alpha_{n}$, an $n$-ary nexus, viz., the nexus associated with $\pi$, and can accordingly replace $\pi$ in any context in which the latter can be substi- 
tuted. On the other hand, if it is logically false, then its negation (or dual of its contradual) is logically true, in which case if $\pi$ does not occur in $\phi$ (and if it does, replace its first occurrence in (CP) by an $n$-place predicate constant which does not) then the substitution of $\phi$, with respect to the variables $\alpha_{1}, \ldots, \alpha_{n}$ for $\pi$ in this negation will result in a logical falsehood, thus showing that substitution in general does not preserve logical truth. Of course, if (CP) is logically false for any $n$-place predicate constant $\pi$, then $\phi$, with respect to $\alpha_{1}, \ldots, \alpha_{n}$, does not represent an $n$-ary nexus. (It might however still represent an $m$-ary nexus for some $m \neq n$.) But whether logically true for one such constant or logically false for all (those or the complements of those occurring in $\phi$ ), in either case the distinction between its formal and its material content is actually shown in the propositional form itself through its substitutional role in the proper logistic of the formal ontology.

Note that even when $\phi$ is an elementary propositional form $(\mathrm{CP})$ is still logically false if $\pi$ does not occur in $\phi$, which of course is precisely as it should be for the logistic factor distinguishing predicate constants from one another. In addition, if $\phi$ is an identity or nonidentity formula, then $(\mathrm{CP})$ is still appropriately logically false since, despite their role as 'prime' formulas, identity and nonidentity statements do not represent or 'picture' an atomic situation. Identity and nonidentity formulas are not, in other words, elementary predicational forms.

\section{The Problem of Identity in Logical Atomism}

Let us now take up the question of our allowing the identity and nonidentity signs as primitive logical constants (of every abstract logical space). As such primitives, these signs generate noncomplex propositional forms and in that regard mimic the grammatical role of (complementary) 2-place predicate constants. But grammatical role aside, identity and nonidentity are not material relations in the sense in which the latter are external conditions of objects (cf. [TR], 5.5301); that is, identity and nonidentity formulas, as we have said, do not represent or 'picture' atomic situations. This latter 'ontological fact', however, cannot be shown by grammar alone, but requires the logistic behavior of that grammar as well, i.e., the proper logicosyntactical employment of the signs for identity and nonidentity. That logistic behavior, we should 
note, is appropriately mirrored in the abstract semantics described above. $^{24}$

According to the Tractatus, the identity sign, and therefore the nonidentity or difference sign as well, "is not an essential constituent of conceptual notation (Begriffsschrift)" (5.533). But that is because in the notational scheme of the Tractatus identity and difference are expressed by an identity and difference of signs and not by means of signs for identity and difference (cf. 5.53). Thus, in the case of names, different Tractarian names are to refer to different objects. Names, however, belong to the application of a logic or formal ontology and are not essential to the characterization of the logical and ontological structure of the world (cf. [TR], 5.526).

But then names are not really essential to the Tractarian account of identity and difference either. For that account is applied to individual variables as well, at least in so far as they occur within the scope of quantifiers interpreted in an exclusive manner. And it is just such exclusive quantifiers that the Tractatus takes as logical primitives (cf. 5.532, 5.5321). Thus, for example, where ${ }^{x} \forall$ and ${ }^{x} \exists$ are the exclusive universal and existential quantifiers, $\pi$ is a monadic predicate constant and $\alpha, \beta$ are distinct individual variables, the propositional sign

$$
\left({ }^{\mathrm{x}} \exists \alpha\right) \pi(\alpha) \wedge\left({ }^{\mathrm{x}} \forall \alpha\right)\left({ }^{\mathrm{x}} \forall \beta\right)[\bar{\pi}(\alpha) \bigvee \bar{\pi}(\beta)]
$$

says that one and only one object is monadically configured by the nexus associated with $\pi$ (ibid.). In our notation with the inclusive quantifiers this proposition requires the identity sign for its expression.

In general, the translation from the exclusive to the inclusive quantifiers is given by the following schemas:

$$
\begin{aligned}
& \left({ }^{\mathrm{x}} \exists \alpha\right) \phi: \exists \alpha\left(\alpha \neq \beta_{0} \wedge \ldots \wedge \alpha \neq \beta_{n-1} \wedge \phi\right), \\
& \left({ }^{\mathrm{x}} \forall \alpha\right) \phi: \forall \alpha\left(\alpha \equiv \beta_{0} \vee \ldots \vee \alpha \equiv \beta_{n-1} \vee \phi\right),
\end{aligned}
$$

where $\beta_{0}, \ldots, \beta_{n-1}$ are all the distinct individual variables occurring free in $(\exists \alpha) \phi$ (cf. Hintikka, "Identity, Variables, and Impredicative Definitions," ([IVID]), 230f). It is obvious, as the translation shows, that identity and difference are implicitly contained within the Tractatus's use of the exclusive quantifiers.

Related to its exclusion of the identity and nonidentity signs as logical 
constants, the Tractatus proclaims all pseudo-propositions regarding the total number of objects in the world to be nonsensical (unsinnig) rather than merely senseless (sinnlos) the way tautologies and contradictions are (cf. 4.1272). This is false doctrine if by nonsensical is meant that such pseudo-propositions cannot be expressed in the propositional forms of the notational scheme of the Tractatus itself. ${ }^{25}$ For example, for each positive integer $n$, let $\alpha_{1}, \ldots, \alpha_{n}$ be the first $n$ distinct variables and let $\phi\{n\}$ be any quantifier free propositional form in which the identity and nonidentity signs do not occur and in which $\alpha_{1}, \ldots, \alpha_{n}$, but no other variables, occur (free). Note that so long as an abstract logical space is not empty there is always such a propositional form of that space, whether it be a conjunction of elementary propositional forms if all the predicate constants of that space are of a degree less than $n$ or is itself an elementary propositional form if the space contains a predicate constant of a degree greater than or equal to $n$. Let us observe now that the following propositional sign expresses the pseudo-proposition that there are at least $n$ objects in the world:

$$
\left({ }^{\mathrm{x}} \exists \alpha_{1}\right) \ldots\left({ }^{\mathrm{x}} \exists \alpha_{n}\right)(\phi\{n\} \bigvee \sim \phi\{n\}) .
$$

In our notation with the inclusive quantifiers and the identity and nonidentity signs as primitive, this pseudo-proposition is expressed by: $(\operatorname{Min}\{n\}) \exists \alpha_{1} \ldots \exists \alpha_{n}\left(\alpha_{1} \not \equiv \alpha_{2} \wedge \ldots \ldots \wedge \alpha_{n-1} \not \equiv \alpha_{n}\right)$.

Naturally, in an abstract logical space in which there are at least $n$ objects, this pseudo-proposition is logically necessary as is easily seen in the Tractarian notation. Accordingly, the propositional sign

$$
\operatorname{Min}\{n\} \rightarrow \square \operatorname{Min}\{n\}
$$

is logically true.

In regard to expressing the pseudo-proposition that there are at most $n$ objects in the Tractarian notation, we need only deny that there are at least $(n+1)$ many objects (but note in passing that this requires $(n+1)$ many variables):

$$
\left({ }^{x} \forall \alpha_{1}\right) \ldots\left({ }^{x} \forall \alpha_{n+1}\right)(\sim \phi\{n+1\} \wedge \phi\{n+1\}) .
$$

This propositional sign is not contradictory, despite appearances, and this is because the use of the exclusive quantifiers renders the sign vacu- 
ously true (if expressible at all) when there are fewer than $(n+1)$ many objects. In our notation, this pseudo-proposition is expressed by:

$(\operatorname{Max}\{n\}) \forall \alpha_{1} \ldots \forall \alpha_{n+1}\left(\alpha_{1} \equiv \alpha_{2} \vee \ldots \ldots \ldots \vee \alpha_{n} \equiv \alpha_{n+1}\right)$

Naturally, this pseudo-proposition is also logically necessary in any abstract logical space in which there are at most $n$ objects, and therefore

$$
\operatorname{Max}\{n\} \rightarrow \square \operatorname{Max}\{n\}
$$

is logically true.

Conjoined together, the two propositional signs, whether of the Tractarian notation or ours, express the pseudo-proposition that the total number of objects in the world is $n$. Naturally, if that is the case then it is logically necessary; that is,

$$
\operatorname{Min}\{n\} \wedge \operatorname{Max}\{n\} \rightarrow \square(\operatorname{Min}\{n\} \wedge \operatorname{Max}\{n\})
$$

is logically true. Needless to say but the same holds for the negation (the dual of the contradual) of this pseudo-proposition, in which case it is in any world of any abstract logical space either logically necessary or logically impossible; and, accordingly, it is therefore either logically necessary or logically impossible in the one real world of the one real logical space based upon reality. Consequently, while such a pseudo-proposition is not 'nonsensical', being expressible in the propositional forms of nominalist logical atomism, it is nevertheless 'senseless' the way tautologies and contradictions are.

In regard to the pseudo-proposition that the total number of objects in the world is infinite, this will be shown in the infinite set of propositional signs of the form $\operatorname{Min}\{n\}$, where $n$ is a positive integer. As represented by such an infinite set, this pseudo-proposition is 'nonsensical' as well as 'senseless'. But we should note that for any nonmonadic predicate constant there is always a complex propositional sign $\phi$ containing that constant that is true in a possible world of an abstract logical space $S$ only if the totality of objects in $S$ is infinite. If modal free, $\phi$ will also be false in other worlds of $S$, but $\diamond \phi$ will in any case be valid in $S$. Naturally, $\sim \nabla_{\phi}$ will be valid instead in any space with only finitely many objects. Accordingly, $\diamond \phi$ will in effect express the pseudo-proposition that there are infinitely many objects in the world, in which case this pseudo-proposition is not 'nonsensical' after all. Nevertheless, although it seems plausible that 
in reality there are nonmonadic atomic situations, we note that if not, then only the above infinite set will suffice.

Before concluding this section, we should mention the one circumstance in which our grammar with inclusive quantifiers and the identity and nonidentity signs as primitive goes beyond that of the Tractatus. That is the case of the one abstract logical space that is empty. For in this one space there are no predicate constants and therefore no elementary propositional forms. And according to the logical grammar of the Tractatus there therefore would be no propositional signs at all. According to our grammar, however, there still are the propositional signs based upon identity and nonidentity formulas, though of course only pseudopropositions are expressed by such signs. In particular, the pseudo-proposition that there are no objects at all is expressed by the propositional sign $\sim \exists \alpha(\alpha \equiv \alpha)$, where $\alpha$ is an individual variable. Though it is a propositional sign of every abstract logical space, it is, at least according to our abstract semantics, valid only in the empty space. The Tractatus, on the other hand, prohibits it outright as nonsensical, though the argument for this claim is rather opaque (cf. 5.5352).

An empty abstract logical space, we agree, has no ontological structure, since such a structure can only be based upon nexuses and, in logical atomism, there are nexuses only in so far as there are atomic situations. Nevertheless, if logical space is allowed to be empty at all in the ontology of logical atomism, i.e., if an empty reality is at all allowable here, then, even if it must be void of an ontological structure, it would still seem to have the logical structure represented by the propositional forms of the empty abstract logical space. Otherwise, in what sense is the empty reality a reality at all? Of course, these propositional forms cannot themselves 'exist' in an empty reality, nor could the pseudo-propositions they express ever be thought in such either, since a thought, as an occurrence, must involve the occurrence of atomic situations. Nevertheless, why cannot thought have a structure even in a reality where it can have no material content and where there cannot be a thought?

We shall not dwell on the issue here. For a careful discussion of the question whether there could or could not be such propositional forms and their associated logical scaffolding were reality void (of atomic situations) would launch us well into a theory of thought for atomism that we have already agreed we would not discuss in this paper. The problem 
does bring one point to the fore, however, and that is that regardless of whether we utilize exclusive quantifiers or inclusive quantifiers with the identity and nonidentity signs as primitive, the logical scaffolding of the world, even if restricted to a nonempty reality, is not reducible to the logical scaffolding represented by propositional connectives alone. ${ }^{26}$

\section{Logical Truth versus Logical Necessity}

Let us return finally to the problematic notion of different realities that we have utilized in our definition of logical truth. It is a notion we have expressly allowed only tentatively, realizing its ultimate incoherence within the framework of logical atomism. Nevertheless, in the abstract representation of that framework, it is a notion that is not without its conceptual utility. It does, for example, bring sharply to the fore the sort of penumbral metaphysical assumption which is the basis of the nominalist's talk of dummy predicate letters in the same context as his introduction of predicate constants as syncategorematic signs.

But logical truth itself, in the end, need perhaps not really presuppose this notion of different realities. For if we were to succeed in the purpose of its construction, viz., to guide us in the characterization of a complete logistic for nominalist logical atomism, though, of course, one in which the logical truth of a propositional sign concurred with the logical necessity of the proposition expressed by that sign, then we need only redefine logical truth there as what is provably or logistically warranted by the system. ${ }^{27}$ And if logical truth did concur with logical necessity, then the entire edifice of our abstract metalanguage might in that case be kicked away as the Tractarian ladder it really is.

As we shall see, however, the set of logically true propositional forms is not recursively enumerable; and, accordingly, if the logistically warranted propositional forms of the formal ontology must be recursively enumerable, then there can be no such descent to a logistically complete system for nominalist logical atomism, or, a fortiori, for realist logical atomism either. And in that case, what appears to be 'said' in terms of logical truth as characterized here cannot be shown in the propositional forms of the formal ontology after all. This conclusion, we should be careful to note, depends on whether the provable or logistically warranted 
propositional forms must be recursively enumerable, that is, whether they must constitute the range of a recursive function.

On the other hand, logical truth could succeed in its purpose only if it concurred with logical necessity. For what can logical truth be but invariant truth in all possible worlds. And in atomism there are no worlds or realities other than the possible worlds that are resolvable into the same atomic situations that are constitutive of reality itself. There are, in other words, no transcendental operators to transmit us to realities with more or less substance than there already is in the world. And yet of course that is just what logical truth as characterized above presupposes. For a propositional sign expressing a pseudo-proposition regarding the total number of objects in the world is neither logically true nor logically false, though the pseudoproposition is itself either logically necessary or logically impossible.

In effect then, what logical truth as based upon different realities introduces is precisely the kind of secondary or restricted interpretation for quantification over objects that we already ruled out for possible worlds. Only, instead of allowing the secondary or restricted interpretations for objectual quantification to apply to the different worlds of one and the same abstract logical space, they are applied globally to the different abstract logical spaces within the abstract supralogical space of our metalanguage. In this way, such quantification, along with identity and difference, is indirectly imputed to have material instead of merely formal content, and it is on the basis of just such indirectly introduced material content that pseudo-propositions regarding the total number of objects in the world are said to be ontologically contingent. Nevertheless, it is not, of course, the sort of material content that can be accounted for by the theory of predication of logical atomism, and in that regard the abstract framework of different realities must be rejected as inappropriate for logical atomism.

Nevertheless, we may still find some positive conceptual utility in our abstract semantics, including logical truth as characterized here. For example, instead of this notion of logical truth, consider the more restricted notion of validity with respect to a cardinal number $k \leqslant \aleph_{0}$, according to which a propositional form $\phi$ is said to be valid with respect to $k$ iff $\phi$ is valid in every abstract logical space of which it is a propositional form and in which the total number of objects is $k$. Naturally, relative to this semantical notion, pseudo-propositions regarding the total number of objects in the world are no longer 'contingent', and objectual quantification, 
identity and difference are accordingly no longer indirectly imputed to have material instead of merely formal content. In addition, and again precisely because of the independence of noncomplementary atomic situations, a propositional form will be valid with respect to a cardinal $k \leqslant \aleph_{0}$ iff it is valid in some logical space of which it is a propositional form and in which the total number of objects is $k$. Accordingly, since some number $k$ is the total number of objects in the world, and, furthermore, since a propositional sign is valid in the one real logical space that is based upon reality iff the proposition expressed by that sign is logically necessary (in the world), then validity with respect to $k$ abstractly characterizes the logical structure of reality, and concurrence with that notion of validity is to be our criterion for logistic completeness. For because of that concurrence we need not in the end step outside of the world in order for a proposition to show its formal or internal 'properties' and to thereby show the distinction between its formal and material content.

We do not know of course what the total number of objects in the world really is, and to that extent our abstract semantics remains conceptually operative. For not knowing that number we can still consider the propositional forms that are valid with respect to every cardinal $k \leqslant \aleph_{0} .{ }^{28}$ And, appropriately, that semantic invariance coincides exactly with the invariance we have called logical truth. Thus, logical truth does concur at least with the logical necessity of those propositions of reality that are independent of pseudo-propositions regarding the total number of objects in the world. And perhaps in this sense logical truth need not presuppose the notion of different realities. But we should note in this regard that there does remain the question whether the propositional signs that are logically true can be separately enumerated and logistically distinguished from those that are valid with respect to the total number of objects in the world. For if they cannot be so distinguished, then in what sense can we maintain that the formal 'property' of propositions that corresponds to the logical truth of their signs is shown in those propositions independently of the notion of different realities?

\section{The Incompleteness of Nominalist Logical Atomism}

Let us turn then to the question of a complete logistic for nominalist logical atomism. Clearly, if the number of objects in the world is finite, then 
that logistic is not only complete but decidable as well. For a propositional form contains only a finite number of predicate constants and to decide its validity with respect to a finite cardinal $k$, we need only consider an abstract logical space that, aside from its total number of objects being $k$, is based upon just the predicate constants occurring in the propositional form. There are only finitely many atomic situations in such a space and therefore only finitely many possible worlds to consider.

But if the total number of objects in the world is infinite, then the set of valid propositional forms is not only not decidable but not recursively enumerable either. For example, where $\phi$ is a modal free propositional sign that is not logically true and in which neither the identity nor nonidentity signs occur, then, by the Löwenheim-Skolem theorem, $\sim \phi$ is true in some possible world of an abstract logical space with $\aleph_{0}$ many objects, and therefore $\diamond \sim \phi$ is valid with respect to $\aleph_{0}$. Accordingly, if the propositional forms that are valid with respect to $\aleph_{0}$ were recursively enumerable, then so would the nonlogical truths of standard predicate logic. But as the latter are not recursively enumerable, then neither are the former ${ }^{29}$

It is noteworthy that for essentially the same reason the set of logically true propositional forms is also not recursively enumerable, as noted earlier. For example, where $\phi$ is a modal free propositional sign in which neither the identity nor nonidentity signs occur and $\psi$ is a propositional sign such that $\diamond \psi$ is valid with respect to $\aleph_{0}$ but $\sim \diamond \psi$ is valid with respect to every finite cardinal, then $(\sim \diamond \psi \vee \diamond \sim \phi)$ is logically true iff $\phi$ is not logically true. ${ }^{30}$ For if $(\sim \diamond \downarrow \vee \diamond \sim \phi)$ is logically true, then it is valid in an infinite abstract logical space $S$, and therefore, by hypothesis, $\diamond \sim \phi$ must also be valid in $S$, which would be impossible if $\phi$ were logically true. On the other hand, if $\phi$ is not logically true, then, as observed above, $\diamond \sim \phi$ is valid with respect to $\aleph_{0}$, in which case so is $(\sim \diamond \psi \vee \diamond \sim \phi)$; but, since by hypothesis $\sim \nabla_{\psi}$ is valid in every finite space, then so is $(\sim \diamond \psi \vee \diamond \sim \phi)$, and therefore the disjunction is valid in every abstract logical space, i.e., it is logically true. Consequently, if the set of logically true propositional forms were recursively enumerable, then so would the set of nonlogical truths of standard predicate logic. But, again, as the latter are not recursively enumerable, then neither are the former.

We should perhaps note in this context that logical truth and validity with respect to $\aleph_{0}$ are equivalent conditions for modal free propositional signs in which the identity and nonidentity signs do not occur. Accord- 
ingly, if the total number of objects in the world is infinite, then the logical truth of these propositional signs concurs with the logical necessity of the propositions expressed. Also, as is well known, the logically true propositional signs that are modal free are recursively enumerable. In other words the only logically true propositional signs that are not recursively enumerable are those that express modal pseudo-propositions, especially those of the form $\diamond \sim \phi$, where $\phi$ is a modal free propositional sign in which neither the identity nor nonidentity signs occur and which is not itself logically true.

We should not conclude from this result that the logistic for nominalist logical atomism is essentially incomplete if the total number of objects in the world is infinite and that therefore our abstract semantics represents content of a sort that can neither be said nor shown in the propositional forms of the formal ontology. For that conclusion presupposes that the logistic must be finitistic or recursively specifiable, i.e., that the provably or logistically warranted propositional forms are recursively enumerable. ${ }^{31}$

But why must the logical structure of the world be finitistic or recursively specifiable in this sense? Is it because it is also the structure of thought or of language? If so, then why must the latter be finitistic in this way? That is, why must it have a structure that is recursively axiomatizable? If it is a matter of the psychological limitations of human cognition, then are we not confusing logic with psychology? Logic sets the limits to the world. Why should the propositional forms that determine that limit be recursively enumerable, i.e., why must they constitute the range of a recursive function? ${ }^{32}$ Why, in particular, must a logistic the avowed function of which is to mirror the logical structure of the world not include infinitistic rules if these rules are valid with respect to that structure ${ }^{33}$

Whatever our ultimate position on this issue might be, it is noteworthy that if there are in the world only monadic atomic situations, that is, if there are only material properties and no material relations, then the set of propositional signs that are logically true is not only recursively enumerable but decidable as well. ${ }^{34}$ This contrasts strikingly with Kripke's result in "The Undecidability of Monadic Modal Quantification Theory," ([Und]), that monadic modal predicate logic is not decidable. Significantly, however, that result essentially requires the allowance of secondary or restricted interpretations of the notion of all the possible worlds of an abstract logical space and, similarly, that the rule of substitution for predi- 
cates is to be extended to all the propositional forms regardless of whether they represent a nexus or not. In this way, as we have already indicated, the modal operators no longer signify merely formal operations but take on material content so that material relations become representable by modal monadic contexts. And, of course, once material relations arc representable by modal monadic propositional signs, then so is infinity and its negative implications for decidability. ${ }^{35}$

Thus, given Kripke's example of associating a modal monadic context $\diamond[\pi(\alpha) \wedge \sigma(\beta)]$ with a relational context $\rho(\alpha, \beta)$, where $\pi, \sigma$ are distinct 1 -place predicate constants, $\rho$ is a 2-place predicate constant, and $\alpha, \beta$ are distinct individual variables, then a pseudo-proposition that there are infinitely many objects and which is expressible by a propositional form that is satisfiable only in a possible world of an infinite abstract logical space, e.g.,

$$
\begin{aligned}
& \forall \alpha \exists \beta \rho(\alpha, \beta) \wedge \forall \alpha \bar{\rho}(\alpha, \alpha) \wedge \forall \alpha \forall \beta \forall \gamma[\bar{\rho}(\alpha, \beta) \vee \\
& \vee \overline{\boldsymbol{\rho}}(\beta, \gamma) \vee \rho(\alpha, \gamma)],
\end{aligned}
$$

becomes expressible by a modal monadic propositional sign. This pseudo-proposition is not expressible by a modal free monadic propositional sign since no such sign is satisfiable only in a possible world of an infinite abstract logical space. Indeed, it is well-known that a modal free monadic propositional sign is logically true iff it is valid in every finite domain, that is, iff it is valid with respect to every cardinal $k<\aleph_{0}{ }^{36}$ Accordingly, any substitution of a modal free monadic context, e.g., $[\pi(\alpha) \wedge \sigma$ $(\beta)]$ for the relational context, e.g., $\rho(\alpha, \beta)$ in the above, of a propositional sign that is satisfiable only in a possible world of an infinite abstract logical space results in a propositional sign that is not only false but logically false as well. ${ }^{37}$ Substituting a modal monadic context on the other hand, e.g., $\diamond[\pi(\alpha) \wedge \sigma(\alpha)]$, results in a 'consistent' propositional sign iff the modal operator is allowed a secondary or restricted interpretation to the effect that not all of the atomic situations configured by the nexuses associated with $\pi$ and $\sigma$ are logically independent of one another. Thus the second conjunct resulting from the substitution of $\diamond[\pi(\alpha) \wedge$ $\sigma(\beta)]$ for $\rho(\alpha, \beta)$ in the above expression is $\forall \alpha \sim \diamond[\pi(\alpha) \wedge \sigma(\alpha)]$, i.e., $\forall$ $\alpha \square[\bar{\pi}(\alpha) \bigvee \bar{\sigma}(\alpha)]$, which states that any pair of atomic situations configured by the nexuses associated with $\pi$ and $\sigma$ and with the same constituent ob- 
ject are logically exclusive of one another, a proposition that is logically impossible in logical atomism. Indeed, precisely because the modal operators are not allowed a secondary or restricted interpretation in logical atomism, the substitution of any monadic context, modal free or not, for a relational context in a propositional sign that is satisfiable only in a possible world of an infinite abstract logical space is logically false.

We do not question here the significance of the metaphysical thesis that all material relations are reducible to material properties. As we have indicated, however, it is a thesis that requires a formal ontology in which modal operators have material and not merely formal content. In that regard, it would seem to be philosophically incoherent that the modal operators involved should represent logical possibility or necessity, or for that matter any logical modality at all. They might of course represent nonlogical modalities, e.g., causal or perhaps even temporal modalities. But in that case the theory of predication as well as the theory of logical form and the ontology purportedly represented thereby are quite alien to the corresponding theories and ontology of logical atomism. In any case, here we see but one amongst many other examples already implicitly suggested in this book of how logical atomism, aside from its own intrinsic value as a formal ontology, serves as a paradigm by which we might better understand the coherence of alternative frameworks.

\section{Notes}

1 The convention adopted here is to use scare-quotes when speaking of what connectives represent as 'properties' or 'relations'. This is done to mark a special philosophical use which is convenient in our informal discussion but which strictly speaking is ontologically misleading. A similar convention applies throughout when we refer to existence (being-the-case) and nonexistence (being-not-the-case) as material 'properties' of atomic situations.

2 A syntactic-semantic ladder for a proposed formal ontology, whether for the framework of logical atomism or that of some other ontology, can be thrown away after a semantic ascent only if what is 'said' on such a ladder can at least be shown, if not said directly by the expressions of the formal ontology, given of course their proper logicosyntactical employment.

3 That is, an object's self-identity or nonidentity with any other object is invariant through all the possible worlds of a logical space containing that object. We must distinguish this ontological invariance from the varying semantical relation of denotation (Bedeutung) between an object and a (non-Tractarian) name or definite description of 
that object. The former must be accounted for within the formal ontology, the latter only within its applications.

4 Russell, in his letters to Wittgenstein, "once asked whether the negations of elementary propositions were themselves elementary propositions, and received the indignantsounding rejoinder: 'Of course not'." Anscombe [2], 34.

5 This syncategorematicity of exemplification in a formal ontology in which properties and relations are individuals is not as arbitrary as it might seem. For even if by means of a comprehension principle logical operations were to be given ontological efficacy in the generation of complex material properties-and a purported iteration of exemplification relations could hardly be said to remain 'simple' in structure-it can be proved that exemplification is not a material relation but only a syncategorematic tie. Cf. Cocchiarella [6].

6 Cf. Bergmann [3] and Hochberg [12] for a presentation of this variant of realism. Hochberg's arguments for negative facts apply equally well for the nominalist and alternative realist positions described below.

7 The use of '(onto)logical' throughout this paper is intended as a reminder that though nexuses are logical structures they are also ontological structures with material content and in that regard constitute the basis of the ontological structure of the world as well as the basis of its logical scaffolding. Unfortunately, nominalists too often note only the logical aspect of nexuses and ignore their material content. This may in part be a consequence of their rather cavalier presentation of predicate constants as syncategorematic signs.

8 Copi in [7] 183, claims that "some properties are definitely compound." In that context, however, Copi is arguing that material properties, unlike material relations, are not nexuses, that is, that there can be no monadic atomic situations. His own view is that material properties "can best be understood . . . as contingent or empirical properties" (p. 182), but he nowhere explains how such entities (with material content!) are to be fitted into the ontology of (nominalist) logical atomism. In regard to their status as nexuses, Sellars in [NAS] shows clearly how material properties as the nexuses of monadic atomic situations are no more puzzling than material relations as the nexuses of nonmonadic situations. In regard to their ontological status, compound properties, as well as compound relations, are the conceptual counterparts of compound propositions and in that regard they figure in atomism's theory of thought or meaning and not, or at least not directly, in its ontology.

9 Cf. Furth [9] for a discussion of the related issuc in Frege's system. The functions that are properties and relations for Frege would be the counterparts of the nexuses of this variant of realist atomism except for the fact that such functions are in general represented by complex and not only elementary propositional forms. Indeed, Frege gives no special ontological significance to elementary propositional forms at all; e.g., his rule of substitution allows any complex propositional form to be substituted for elementary forms (subject only to the usual constraints of clash of bound variables).

10 In so-called presupposition-free logics, the two notions are allowed to diverge and free variables represent a wider substitution class of individual expressions than bound vari- 
ables. However, given the usual unrestricted form of the rule of substitution (of open formulas for predicate expressions), it is not clear that norninalism in any form can coherently allow itself the use of such a logic, especially when extended to modal logics where the unrestricted rule of substitution belies the material content of modal propositions as well as of objectual nonexistence. It is no wonder then that objectual existence is generally represented in such a logic by an elementary predicational form, e.g., ' $E !(x)$ '.

11 One might think to exclude those possible worlds of the logical space in which some nexus fails to be the nexus of any existing situation of that world. Such an exclusion, however, as with any restriction placed upon the notion of all the possible worlds of a logical space, incorporates, as was shown in [5], material content into the modal structure of propositions. Cf. sections 3 and 4 below.

12 Allair in [1], 336, notes the 'unsaturation' or incompleteness of both objects and material properties, but draws the extraordinary conclusion that both are therefore the same in kind, and in particular that both are objects.

13 The sense of this 'can' belongs to general, comparative ontology and not to the logical possibility of logical atomism.

14 It is presumed here that the natural numbers, integers or rationals, the cardinality of each class of which is $\kappa_{0}$, are not entities of an ontological type different from that of the real numbers. That is, each is but a kind of real number, and in that regard all are abstract entities. In any case they cannot be less than $\aleph_{0}$ in number, and therefore do not pass the test of being concrete.

15 That is, where $\pi, \sigma$ are different $n$-place predicate constants and $\alpha_{1}, \ldots, \alpha_{n}$ are distinct individual variables, $\sim \square \forall \alpha_{1} \ldots \forall \alpha_{n}\left[\pi\left(\alpha_{1}, \ldots, \alpha_{n}\right) \leftrightarrow \sigma\left(\alpha_{1}, \ldots, \alpha_{n}\right)\right]$ is to be logically true.

16 It should be emphasized that this replacement of nexus identity by predicate identity is a consequence of nominalism, not of a Tractarian thesis that an identity and difference of entities is to be replaced by the identity and difference of their signs. Within the second variant of realist logical atomism, on the other hand, and despite its agreement with nominalism otherwise, there is an expressible form of nexus identity and difference that is independent of the occurrence of predicate constants, viz., coextensivity in all possible worlds.

17 The claim here is not that 'no predicate expression is an individual or nominal expression' says that no nexus is an object. Rather, in the formal ontology of atomism, the grammatical fact that no predicate constant is an individual or nominal expression shows what in the surface grammar sentence of English appears to be said by 'no nexus is an object'. That this can only be shown and not said is all the more obvious when the grammar is 'perspicuous'.

18 Compare the analogous constraint in so-called free logics: only a term referring to an existent can be substituted for a generalized variable, or, equivalently, only such a term 'comprehends' an individual. Thus the universal instantiation law here takes the form:

$$
\exists \alpha(\xi=\alpha) \rightarrow(\forall \alpha \psi \rightarrow \psi(\xi / \alpha))
$$


Similarly, since nexus 'identity' is expressed by necessary equivalence, the universal instantiation law in this variant of realist logical atomism takes the form:

$$
\exists \pi \forall \alpha_{1} \ldots \forall \alpha_{n} \square\left[\phi \leftrightarrow \pi\left(\alpha_{1}, \ldots, \alpha_{n}\right)\right] \rightarrow\left\langle\forall \pi \psi \rightarrow \psi\left(\varphi / \pi\left(\alpha_{2}, \ldots, \alpha_{n}\right)\right),\right.
$$

where $\pi$ is an $n$-place predicate or nexus variable not occurring (free) in $\phi$, and $\alpha_{1}, \ldots$ ,$\alpha_{n}$ are among the distinct individual variables occurring free in $\phi$. The antecedent here stipulates that $\phi$, with respect to the variables $\alpha_{1}, \ldots, \alpha_{n}$ as argument indicators, represents an $n$-ary nexus. Such a nexus of course, as well as the atomic situations it generates, can be represented by complex as well as by atomic propositional forms. Cf. ([TR], 4.465), "The logical product of a tautology and a proposition says the same thing as the proposition."

19 That is, the rule that from the logical truth of a propositional form $\psi$, the logical truth of $\psi\left(\varphi / \pi\left(\alpha_{1}, \ldots, \alpha_{n}\right)\right)$ follows, provided that $\phi$, with respect to the variables $\alpha_{1}, \ldots, \alpha_{n}$ as argument indicators, represents an n-ary nexus.

20 Of course, the pseudo-proposition that there is no such nexus of a certain degree will not be formulable for even the realist if it be demanded that each $n$-place predicate or nexus variable of his formalism be itself an $n$-place (linguistic) nexus.

21 We restrict our considerations in this paper to propositional forms of finite length. Atomism does allow for atomic situations with infinitely many constituents and these can be represented or 'pictured' in a formalism with expressions of infinite length. Whether the Tractarian theory of thought or philosophy of language can account for these infinite situations as thinkable elementary propositions is of course another matter altogether.

22 Whether logical atomism can coherently allow these developments at a more superficial level, especially at the level appropriate for the grammatical analysis of ordinary language, will depend greatly upon the theory of thought or philosophy of language to be appended to its formal ontology. It is not clear that the theory of the Tractatus is the only one appropriate to the ontology of logical atomism.

23 In the case of substituting $n$-place predicate constants for $n$-place predicate constants the rule is: if $\psi$ is logically true, $\pi, \sigma$ are distinct $n$-place predicate constants and neither $\sigma$ nor its complement occurs in $\psi$, then $\psi(\sigma / \pi)$ is logically true. The qualification that neither $\sigma$ nor its complement occurs in $\psi$ is required since, for example, logical truth is not preserved in the substitution of $\sigma$ for $\pi$ in the logical truth $\exists \alpha_{1} \ldots \ldots \exists \alpha_{n}$ $\diamond\left[\pi\left(\alpha_{1}, \ldots, \alpha_{n}\right) \wedge \bar{\sigma}\left(\alpha_{1}, \ldots, \alpha_{n}\right)\right]$. This last propositional sign is logically true since in the context of nominalist logical atomism different predicate constants necessarily represent different nexuses, and, accordingly, the qualification is quite appropriate for this very reason. (Incidentally, it should not be thought that since it is logically true the above existential propositional sign is therefore valid in the empty logical space. No existential propositional sign is valid in the empty space. But, then, because of the occurrence of predicate constants, the above is a propositional sign only of a nonempty abstract logical space, and it is clearly valid in every abstract logical space of which it is a propositional sign.)

24 That $\equiv$ and $\not \equiv$ do not represent binary nexuses is reflected in their nonsubstitutability 
for 2-place predicate constants. Another illustration of this nonsubstitutability is the following:

$$
\begin{aligned}
& \forall \alpha \forall \beta \diamond \rho(\alpha, \beta), \\
& \forall \alpha \forall \beta \diamond \alpha \equiv \beta, \\
& \forall \alpha \forall \beta \diamond \alpha \equiv \beta .
\end{aligned}
$$

Here, (a) is logically true, though (b) and (c), the results of substituting $\equiv$ and $\not \equiv$ for $\rho$, are not. Note, incidentally, that (a) is logically true only because the notion of all the possible worlds of a logical space is not allowed a secondary or restricted interpretation.

25 Among the pseudo-propositions we express in ordinary language, we should distinguish between those that are directly expressible in the propositional forms of nominalist logical atomism and those that can only be shown in the formal ontology as a whole.

Those considered so far, e.g., that no nexus is an object, or that nexuses, unlike other logical properties and relations, have an ontological or material structure as well, etc., are not directly expressible in the propositional forms of the framework but are shown either through distinctions in its ontological grammar or through the logistic behavior of that grammar. These pseudo-propositions, to use Tractarian terminology, are 'nonsensical' as well as 'senseless'. Other pseudo-propositions, e.g., that a tautology or contradiction is logically necessary or impossible, respectfully, can be directly expressed by those propositional forms, though modal operators are required for this purpose, and are in that regard not 'nonsensical'; but, because they are either logically necessary or logically impossible, they are 'senseless' and therefore pseudo-propositions.

26 The essential use of Tractarian names and the conjunctive and disjunctive substitutional interpretation of the universal and existential quantifiers has been the principal pitfall for this sort of erroneous view. As already noted, we should avoid mixing the essential characterization of a formal ontology with its applications. In that way the true nature of the logical structure involved will more readily stand out.

27 Compare Quine [14], 297, for precisely this sort of maneuver for the logistic of standard predicate logic. Quine cannot include the modal operators in this maneuver since not being an atomist, he cannot construe possible worlds as resolvable into the atomic situations of reality, and modal operators would otherwise purport to represent material and not merely formal content.

28 There remains the question of whether we can consider all the propositional forms that are valid with respect to a cardinality larger than the total number of objects in the world, since some of these forms will require more variables than there are objects available. We have suppressed problems of this sort throughout the present paper since they properly belong to atomism's theory of thought or philosophy of language.

29 Except for the use of Tractarian names and state descriptions, the semantics of Carnap [4] is essentially that of validity with respect to $\aleph_{0}$. Carnap attempts a proof of completeness there but leaves the question unsettled. 
30 Note that $\diamond \psi$ expresses in effect the pseudo-proposition that there are infinitely many objects. Cf. [10], 135, for the completely analogous result of standard second order logic.

31 The Tractatus we might note, apparently maintains the stronger thesis that 'logical propositions' are decidable. "It is the peculiar mark of the logical propositions that one can recognize that they are true from the symbol alone, and this fact contains in itself the whole philosophy of logic" (6.113) (cf. also 5.551,6.122, 6.125).

32 If what is shown by means of logistic need not be finitistic in the above sense, then why must it be so for what is shown by grammar? For example, might not the grammatical representation of atomic situations of infinite complexity require nonfinitistic grammatical constructions?

33 As an example of such an infinitistic rule, suppose that a Tractarian name has been associated with each of the infinitely many objects in the world. Then from the infinite premise set consisting of all the instances $\phi(\xi / \alpha)$ for each such name $\xi$, the generalization $\forall \alpha \phi$ validly follows. The analogous rule of infinite induction in elementary arithmetic, we might note, renders that system deductively complete rather than incomplete.

34 The proof of this can be seen by translating each propositional form $\phi$ into a formula $t(\phi)$ of standard second order monadic predicate logic (with identity). Essentially the translation replaces each subwff of $\phi$ of the form $\square \psi$ or $\diamond \psi$, where $\psi$ is modal free, by $\forall \pi_{1} \ldots \forall \pi_{k} \psi$, or by $\exists \pi_{1} \ldots \exists \pi_{k} \psi$, respectively, where $\pi_{1}, \ldots, \pi_{k}$ are all the (monadic) predicate constants occurring in $\psi$ (and now reconstrued as monadic predicate variables). It can then be shown that $\phi$ is logically true iff $t(\phi)$ is, and therefore since the logical truths of standard second order monadic predicate logic (with identity) are decidable then so are the monadic propositional forms of nominalist logical atomism.

35 By the translation described in the last footnote, Kripke's result can be used to show that the 'generally valid' formulas of second order monadic predicate logic are not decidable, where the generally valid formulas are those valid in every model, standard or nonstandard. (In the nonstandard models the notion of all properties, or all classes of objects drawn from the domain of discourse, is allowed secondary or restricted interpretations.) This suggests that somehow the allowance of nonstandard models alters the supposedly formal content of predicate quantifiers so that material content becomes expressible through their application.

36 Therefore, if such a propositional sign were satisfiable only in an infinite model or possible world, then its negation would be valid in every finite domain and hence logically true, which is impossible since the propositional sign is satisfiable (in an infinite domain). Because the modal operators are not allowed a secondary or restricted interpretation, a similar argument applies for the modal monadic propositional signs.

37 Thus the first and second conjuncts of the result of substituting $[\pi(\alpha) \wedge \sigma(\beta)]$ for $\rho(\alpha, \beta)$ in the above example, i.e., $\forall \alpha \exists \beta[\pi(\alpha) \wedge \sigma(\beta)]$ and $\forall \alpha \sim[\pi(\alpha) \wedge \sigma(\alpha)]$ are conjointly logically false. 


\section{References}

[1] Allaire, Edwin B., "The "Tractatus": Nominalistic or Realistic?", in [8], 325-431.

[2] Anscombe, E., An Introduction to Wittgenstein's Tractatus (London: Hutchinson and Co., 1959).

[3] Bergmann, G., "Ineffability, Ontology and Method," Phil. Review 69 (1960): 1840.

[4] Carnap, R., "Modalities and Quantification," JSL 11 (1946):33-64.

[5] Cocchiarella, N. "Logical Atomism and Modal Logic," Philosophia 4 (1974): 41 66. Reprinted in the present volume as chapter 6.

[6] Cocchiarella, N., "Properties as Individuals in Formal Ontology," in Noǔs 6 (1972): 165-87.

[7] Copi, I., "Objects, Properties, and Relations in the Tractatus" Mind 67 (1958): 145-68. Reprinted in [8].

[8] Copi, I. and Beard, R. W. (eds.), Essays on Wittgenstein's Tractatus (London: Routledge \& Kegan Paul, 1966).

[9] Furth, M., "Two Types of Denotation," in Studies in Logical Theory, American Philosophical Quarterly, Monograph Series, Monograph No. 2 (Oxford, 1968).

[10] Hasenjaeger, A., Introduction to Basic Concepts and Problems of Modern Logic (Dordrecht, Holland, D. Reidel, 1972).

[IVID] Hintikka, J., "Identity, Variables and Impredicative Definitions," JSL 21 (1956): $225-45$.

[12] Hochberg, H., "Negation and Generality," Noǔs 3 (1969): 325-43.

[13] Kripke, S., "The Undecidability of Monadic Modal Quantification Theory," Zeitschr. f. math. Logik und Grundlagend. Math. 8 (1962): 113-16.

[14] Quine, W., "Replies," Synthese 19 (1968):264-322.

[NAS] Sellars, W., "Naming and Saying," Philosophy of Science 29 (1962):7-26.

[TR] Wittgenstein, L., Tractatus Logico-Philosophicus, D. F. Pears \& B. F. McGuinness, trans., 2d. ed. (London: Routledge \& Kegan Paul, 1971. first ed. 1921). 


\section{Index}

Abelardian thesis, 104, 112

Abelardian view, 102-5, 112

Actualism, 11, 120

Actualism-possibilism debate, 120

Allair, Edwin B., 280n.12

Analytic philosophy, 1-8

Anscombe, E., 279n.4

Antiessentialism, modal thesis of, 14, $18 \mathrm{n} .14$

Atomicity, principle of, 194, 211, 217-22

Atomistic hierarchy of sentences, 16, 19495, 211, 213, 216-19

Ausserkonstitutorisch, 127. See also Properties and relations, extranuclear

Bar-Hillel, Y., 154

Begriffsschrift, 8-9, 160

Being, 119, 121, 126, 134, 205, 234, 244, $247,251,255,261,265$. See also Subsistence

Bergmann, G., 279n.6

Boolos, G., 153, 156

Bradley, F. H., 248

Cantor, G., 28, 33, 38, 252

Carnap, Rudolf, $61 \mathrm{n} .18,65,216,223$, $265,282 n .29$
Chierchia, Gennaro, 17n.7

Choice, axiom of, 156, 174, 175

Church, Alonzo, 55-56, 75, 90-91, 15557, 161, 196-200, 220n.2

Church's characterization of ramified logical types, 54-56, 196, 199

Class

logical notion of, $6,9,152,156,175$, $178,181,190 \mathrm{n} .1$

as many, $21-25,28,59$ n. $1,80,81,89$, 121,156

mathematical notion of, 152, 175, $190 \mathrm{n} .1$. See also Iterative concept of set

as one, $21-23,28,59 \mathrm{n} .1,80$, 81

Classes as composed of their members, 105. See also Class, mathematical notion of

Complementation, 224-26

between atomic situations, 224-26, 246, 248, 251, 258-62, 266, 274

between elementary propositions, 22627,240

between nexuses, 221, 225, 247-52

Complexes, Russell on, 49, 51, 56, 203-4, 206-7, 209, 217-19 
Concept-Correlates, 71, 92-94, 103, 105-

$7,165,167-69,178-82,188-90$

conditions for positing, 178-80, 189

Fregean, 67, 69, 76-80, 153, 160, 169

Russell on, 110

as NF-"sets," 169, 172

as NFU-"sets," 109, 185

value-ranges as, 76-79, 99, 102, 158

Concept formation, 112-13, 136

impredicative, 113, 136

Concepts, 65-71, 100-15

as cognitive capacities, $111-15$

correlation of. See Double correlation thesis

in Frege's sense, 71, 76-80, 98, 104, 159. See also Unsaturatedness

first level, 77-78, 91-92, 106, 157, 159, $165-66,176,178,179,188,189$

higher level (third and above), 92, 97, 102, 106, 157-59, 165, 172, 178,

190n.3

impredicative, 112

as individuals, 101, 109, 112

predicable, 111, 113, 156

as propositional functions, $69,99,153$

referential, 111,112

rigid, 100-2, 168, 190n. 1

as saturated entities, 84

second-level, 61n.17, 77-78, 91-92,

$111,157-59,164$

stratified hierarchy of, 93, 165-66

unsaturated, 94, 99, 103-4, 111, 181-

82

unsaturated, Frege's hierarchy of, 94, 97,

102, 157-59, 165-66, 190n.3

Conceptual Platonism, 111-16

Conceptualism, constructive, 113, 115

holistic, 113, 115, 136, 140

Content

distinction between formal and material, $249,253,262,273-74,278$

formal, 222, 245, 249, 266, 282n.27, 283n.35

material, 222, 245, 249, 255, 265-66, 271, 277, 279n.7, 280n.10, 282n.27, $283 \mathrm{n} .35$

Context principle, Frege's. See Frege's context principle
Contexts of use, 4, 114-15

Continuum hypothesis, Cantor's, 252

Copi, I., 279n.8

Cumulatively, stratified, 93, 144

Definite descriptions, 122-29, 140-41, 279 n.3

Russell's contextual analysis of, $61 \mathrm{n} .18$

Denoting concept, 60n.11, 61n.17, 123, 125. See also Russell, Bertrand, theory of denoting

Doctrine of Showing, 255. See also Zeigen

Double correlation thesis, Frege's, 10, 78$80,87,92-94,102,105-6,152-53$, $157-60,164,167,172,178,187-89$ modifications of, 165-69, 172, 176-81

Essentialism, 245. See also Antiessentialism, modal thesis of

Events, 203-6, 217-19. See also Facts, concrete

Exemplification, 66, 224, 248, 251, 279 n.5. See also Syncategorematic tie

Existence, 11, 120, 129, 132-41, 147, 149, 200, 205-6, 224, 247, 265 of atomic situations, 224, 226, 247, 253 in logical atomism, 226, 228, 231, 248, $251,253,278 \mathrm{n} .1$ predicate, 136, 147

Extensionality, axiom of, 42, 66, 105, 152, 153, 170, 178, 181, 190n.1

Extensionality, principle of, 13, 97-99, 100, 164

Extranuclear properties. See Properties and relations, extranuclear

Euclidean geometry, 128

Facts, 203-10, 217-19

abstract, 203-6

atomic, 13-14, 16, 241, 253-54

complex, 207

concrete, 205-9.

maximal classes of, 15

negative, 247-53, 279n.6

modal, 241

ontological, 267

possible, 251-52

Falsity, logical, 230, 236 
Fictional objects, 147-50

Fracnkel, A., 154

Frege, Gottlob, 8-12, 64-111, 152-60, 250, 279n.9

on nominalized predicates, $80-83$

on Russell's paradox, 10, 83, 90, 102-5, 176

on Schröder, 89-91

Frege's Basic Law V, 76, 79-80, 86, 88, 97-98, 115n.2, 158-59, 164

context principle, $66,78,80$

Functionality, mathematical notion of, 7172

Functionality versus predication, 70-72

Furth, M., 279n.9

Gallin, Daniel, 116n.6

Gettier, Edmund, 115n.1, 145

Gödel, K., 191 n.12

Henkin, Leon, 73, 76, 190n.3

Heterogeneously stratified, 93, 166-67

Hintikka, J., 18n.16, 268

Hochberg, H., 279n.6

Holistic ontology, 265

Homogencously stratified, 93-97, 101, $103,106,109,143-44,166-67,178$, 183

Identity and difference in logical atomism, 246, 273-74

Identity of Nuclear Indiscernibles, axiom of, 130

Identity, problem of, in logical atomism, 267-72

Incomplete Symbols, 49, 53, 62n.19, 100, 125, 196-98. See also Russell's theory of denoting, 1905 or new or theory of incomplete symbols

Individuals, 20, 26, 30, 43-44, 53-55, 59n.1, 65-69, 80, 86, 90-91, 197

abstract, 91, 114, 121, 126, 147, 149, 178

concrete, 114,116 n.5, 120-23, 126, $128,134,147,149,157$

as denoted by nominalized predicates, 92, 95 existing, 129, 131, 133-34, 137, 140, 148-49

hicrarchy of, Schröder's, 89-91, 155

non-existing, 133-34

possible, 100, 120, 123, 129, 148

Russellian, 119-22, 128-29, 133-35, 137, 149-50, 202-3, 208

Infinite regress argument, Bradley's, 248

Infinity, axiom of, $65,75,114,156,174$, $175,189,194$

Intensional logic, 4, 6, 14-25, 120, 122, 134-35

Intensionality, principle of, 100, 109, 114, 168

Iterative concept of set, 5-6, 9, 152-60, $175-78,190 \mathrm{nn} .2,3,205$

Jensen, R., 97, 101, 107, 172, 174-75

Kaplan, David, 238, 243n.14

Kerry, Benno, 76

Konstitutorisch, 127. See also Properties and relations, nuclear

Kriesel, G., 155

Kripke, Saul, 233, 242n.8, 276-77, 283n.35

Lackey, P., 17n.8

$\lambda$-conversion, principle of, 75

Laws of logic, 65-66, 72-76, 90-92, 95, $98,100-2,114,176$

Frege on, 72-73, 98-99, 163

Russell on, 100-1, 168, 194

Leibniz, 132, 164

Leibniz's law, 86

Lésniewski, S., 76

Limitation of size doctrine or theory, 28, $105,154,156,177$

Logic, free, 136, 138, 176, 279n.10, 280n. 18

the system HST ${ }_{\lambda}^{*}, 107$

Logical atomism, 1-5, 12-16, 20, 195, 206-11, 222-84

formal ontology of, 246-47, 253, 255, 262, 266-67, 280n. 17, 281 n.22 nominalist, 222, 225, 235, 238, 243n.12, 244, 246, 249, 255, 257, 260, 270, 272, 279n. $8,28 \ln .23$ 
nominalist, abstract semantics for, 259-

62

nominalist, incompleteness of, 274-78

ontological grammar of, 222, 232, 245, 262-67

ontology of, 223, 246, 271, 278

philosophy of language of, $247,281 \mathrm{n} .22$

realism in, 222, 225, 226, 238, 244, $249,258,272,280-81$

realist with properties and relations as nexuses, 15-16, 223, 225, 252, 256, 279n.9, 280n.16, 281n.18

realist with properties and relations as objects, 15-16, 223-24, 248, 249-50

Russell's form, 206-11

theory of logical form, 244, 257

theory of meaning of, 247

theory of predication of, 223, 246-47, $261,273,278$

theory of thought of, 247, 271, 281n.22

Logical fiction, 210

Logical form, theory of, 1-9, 244, 25758, 278

nominalist theory of, 258

as a regulating ideal, $2-4,10$

Logical grammar of the Tractatus, 271

Logical positivism, 1-5

Logical realism. See Realism, logical

Logical scaffolding, 13-14, 234-44, 253, $255,271-72,279$ n.7

Logical space, 14, 225-33, 238, 265

abstract, 225-27, 260-63, 269-70, $28 \ln .23$

based on and different from reality, 246, $253,260,264,273-74$

of elementary propositions, 227-29, $232-33,237,242 \mathrm{n} .8$

empty, 260, 271, 281n.23

individuated, 260

in nominalism, 252-59

Logical subject, 19-63, 209-10, 219

plural, 23-25

propositional functions as. See Propositional functions as single logical subjects

propositions as, 29-33. See also Propositions as single logical subjects and the univocity of being, 20-23, 34, 39 in Russell's 1908 theory of logical types, $43-48$

single, 36

Logical truth, 229-30, 233, 235-41, 259, 264-67

versus logical necessity, 272-74

Logical types, theory of, 5-8, 19, 29, 56-

$58,91,59$ n. 6,146

ramified, 52-56, 91, 113, 115, 154

r-types, 207-9

simple, 10, 89, 91, 92-94, 146, 154, 157-60, 216

simple, homogeneous, 59n.16, 94-97, $143,146,165-68$

simple as a theory of classes, 153-56, 172,175

Russell's 1903 theory in Appendix B [POM], 10, 23, 25, 59n.6, 89-90, 94, $121,155-57,193,195,216$

Russell's 1908 theory, 43-48, 58, 60n.11, 196-200

Russell's 1910-13 [PM] theory, 193, 196-200. See also Logical types, theory of, ramified

Russell's later or post-[PM], 193-94, 202, 207, 211

Russell's theory in his logical atomism, 206-11

Logically correct language, $2-3$

Logically perfect language, 1, 3, 6, 195, 201, 217

Logicism, 8-11, 64-65, 70, 115, 189

Frege's form, 64-72, 80, 83-84, 101, $104,111,115,152,160$

first reconstruction, 92-93, 104, 166

an intensionalized form, 108-11

as a second order predicate logic with nominalized predicates, $80,99,160-65$

and the predicative nature of concepts, 65-70

Russell's as conceptual platonism, 11115

Russell's early (pre-1905) form, 5, 6471, 80-84, 88, 101, 109, 115, 168

Russell's later form in [PM], 69, 111, 115

a second reconstruction, 105-8

Löwenhein-Skolem theorem, 275 
Mathematical induction, principle of, 41 , 42, 60n.14, 108, 186-90

Meaning, Tractarian picture theory of, 15 , 223, 242n.5

Meaning, theory of, verificationist, 2-3

Meinong, Alexius, 11-12, 60n.12, 11941 ,

Meinongian definite descriptions, 122-29

Meinongian objects, 119-22, 129-33. See also Objects, Meinongian

Modal logic, 120, 122, 167, 262, 266

and logical atomism, 222-43

and logical atornism and nominalism, 244-84

logical atomism as a paradigm of, 253

second order, 135-38

Modes of configuration. See Nexuses

Monad, 132

Montague grammar, 6, 25

Montague, Richard, 116n.6, 191n.6 intensional logic of, 122, 134-35

theory of definite descriptions, 122,125

Moore, G. E., 1

Multiple relations theory of judgment, Russell's, 48-52, 55-57, 60n.12, 19698, 208-9, 213

Multiplicative axiom, 75

Names, Tractarian, 265, 268, 282nn.26, 29,283 n.33

Natural numbers and classes, Russell's analysis of, 39, 41-42, 46, 193-95, 199-201, 211, 216-19

Natural numbers, Fregean, 186-90

Necessity, logical, 14-15, 222-23, 230$33,242 \mathrm{n} .10,245,254,257,259,265$, 278,282 n. 25

Negation, 224, 228

Negative facts. See Facts, negative

Von Neumann-Bernays-Gödel set theory, 22

Nexuses, 224-25, 258, 266-67, 271, 277, 279n. 17, 280n.11, 281

categorial existence of, 255, 258

complementary, 247-52

as formal properties or relations, 255

identified with predicate constants, 260 , $28 \ln .23$ linguistic, 250, 253-54, 281n.20

as material properties and relations, $15-$

$16,223,248-50,253,255,280$ n. 16

number of, $252,258-59$

and objects, 260, 268, 280n.17, 282n.25

structure of, 253-54

as syncategorematic signs, 255, 257, 259

as universals, 252

"No classes”theory. See Russell's "No

classes" theory

Nominalism, 15-16, 222-25, 234-35,

244-84

in logical atomism. See Logical atomism, nominalist

on pseudo-propositions, 256

ontological grammar of, 259

Nominalized predicates, 6-10, 65-70, 80$88,92,109,112-13,116 \mathrm{n} .8,145,160$, 169,209

as denotationless singular terms, 104-5, 176-78. See also Abelardian view in second order logic, 95, 97, 99, 102, $105,116 \mathrm{n} .8,160-65,176$

Non-existence, 11-12, 119-20, 132, 14750, 280n. 10

in logical atomism, 224-28, 231, 253, 247-48, 278n.1

Nuclear properties. See properties and relations, nuclear

Object, the full (Meinongian), 131-33

Object, the null (Meinongian), 130-32

Objects

abstract, 126

concrete, 119, 122, 126, 147, 149

as concrete particulars, 246, 252

contradictory or impossible, 128-29,

$131,133,148$

existing, 132-33, 150

of fictions or dreams. See Fictional objects

in logical atomism, 246-47, 249, 251

Meinongian, 119-22, 126, 129, 130-

$37,141,147,149-50$

Parsons's axiom for, 130

possible, 132

possible, Parsons's definition of, 131 
in Russell's 1910 theory of types [PM], 195, 203, 206

totality of, or total number in the world, $246,252,259,265,269-70,273-74$, 276, 282n.28

One and Many, problem of, 21, 26, 34, 52

Ontological contingency, 259-60

Ontological structure, 14, 244, 253, 268, 271, 279n.7

Ontology. See Realism; Nominalism

Ontology, formal, 223, 234, 241, 244, 250, 252-53, 255, 268, 278nn.2, 3, 279 n. $5,282 \mathrm{n} .26$

nominalistic, 234, 253, 263, 278

Ordinary language philosophy, 1-5

Paradox of the liar, 40, 42

Parsons, Terence, 11, 119-51

Particulars, 203-11

complex, 203-6, 209. See also Events; Complexes, Russellian

concrete, 246, 249-52

Platonic forms, 112, 114

Poincaré, Henri, 38, 60n.10

Possibilism, 7, 11, 116nn.4, 5, 120

Possible worlds, 99, 101, 110, 111, 120,

$126,131-33,137,148,150$

all, 232, 253, 261, 273

all, secondary or restricted interpetation of, 232, 262, 265-66, 273, 276-78, $282 \mathrm{n} .24,283 \mathrm{n} .36$

all, standard notion of, 229, 230, 232, 278

in logical atomism, 14-15, 226, 247, 251-54, 261, 266, 275, 278

logical nature of, 253

of a logical space, 251, 254, 261, 264$66,270,275-78,278 \mathrm{n} .3,280 \mathrm{n} .11$

as maximal classes of propositions, 120

as world facts or world propositions, 15 , 266

Possibility, logical, 14-15, 222-23, 233, 245, 257, 259, 261, 265, 278, 280n.13

Pragmatics, 4, 6, 114

Predication (theory of), 5, 10, 65-66, 145, 156, 167, 170, 178, 244, 278

versus functionality, $70-72$ in logical atomism. See Logical atomism, theory of predication of

Meinongian, 130, 138

nominalist, 257-58

Russellian, 130, 138, 208

Predicational forms, elementary, 266-67

Properties, relational, 141-42

Properties and relations

complex, 201, 225, 249, 279n.5

existence entailing, 133-39, 142, 147, 150

external, 14

extranuclear, 127-30, 133, 135, 137$40,142,147$

extranuclear essentially, 135

formal, 14, 222-23, 226-29, 231-32, 239, 241, 243n.12, 245-47, 259, 265, 274

as individuals, $121,143-47,279 \mathrm{n} .5$

internal, 13, 142, 222-23, 226, 228-34, 236, 239, 241, 243n.12, 245, 256, $259,265,274$

as logical subjects, 57,193

material, 13-16, 222-28, 231, 234, 238-39, 241, 242n.10, 243n.12, 245-51, 253, 256, 265, 267, 277-78, 278n.1, 279n.8, 280n.12

nuclear, 127-42, 149-50

simple, 13, 201, 210-11, 225

Propositional forms (of a formal ontology), 255-56, 271-77, 279n.9, 281n.18, $282 \mathrm{nn} .25,28$

elementary, 254, 258, 263, 269, 271, 279 n.9

Propositional functions

as expressions or linguistic entities, 16, $53,194-95,202,211-17$

first order, 45, 53, 157

as individuals, 81, 89-90, 99. See also

Propositional functions as logical subjects

the level of, 54, 56, 196

as logical subjects or single entities, 21 , 26-29, 45-48, 52-53, 58, 59n.6, 69, 81, 83, 196-200, 202, 217, 219

the logical type of, 45, 53-54

as non-entities, 25-29, 46, 48, 196, 212 
predicative, 26-28, 36-38, 45, 52-54,

59n.3, 197, 198, 205, 220n.3

as properties and relations, 16, 51 ,

61 n. 15, 193-94, 200-2

second order, 53

as universals, 16, 49, 200-4

Propositional sign, 227, 230, 236, 245,

263, 270-77, $281 \mathrm{n} .23$

elementary 226, 234, 243nn.12, 13

Propositions, 38-42, 48-49, 52, 55, 57-

$58,60 \mathrm{nn} .9,11,61 \mathrm{n} .17$

atomic, 57

complex, 236

elementary, 43-44, 51, 223, 227, 23639,279

elementary, logical space of, 227-29, $237,240,242 \mathrm{n} .8$

as facts, 58

first order, $43-45$

as individuals, 43,121

logical, 283n.31

as single logical subjects, 29-33, 49-52,

55-58, 60n.11, 196-99

mathematical, 39, 59n.1

nonsensical (unsimnig), 269-71, 282n.25

of real logical space, 230

second order, 44

senseless, (Sinnlos), 269-70, 282n.25

as sentences or linguistic conveniences, 212

Protokolsätze, Carnapian theory of, 223

Pseudo-propositions or Scheinsatz, 25557, 269-77, 281n.20, 282n.25, 283n.30

Qualities, simple, 218-19

Quantifiers

exclusive, 268-69, 272

inclusive, 268, 271-72

Quine, W., 126, 153, 157, 181, 186-87, $282 n .27$. See also Set theory, Quine's

$\mathrm{ML}$ and $\mathrm{NF}$

Quine's thesis, 169-72, 185, 187, 189

Ramsey, F. P., 153

Realism, 2, 11, 15-16, 235

logical, 1-2, 13, 16, 201, 206 in logical atomism. See Logical atomism, realism in

metaphysical, 1-2, 13, 15

natural, 2, 13, 16

scholastic, 200, 205

Realist-nominalist controversy, logical atomisms's version of, 248

Realities, different, 259, 264, 272-74

Reality in logical atomism, 247, 252, 259, 261, 271-74

Reducibility, axiom of, $28,42,50,53,54$, 59n.3, 65, 114, 115, 140, 153, 194

Relations

equal higher level, 178. See also Homogeneously stratified

inhomogeneous unequal higher level, 92, $94,102,106,166-67,178,188-89$

second level, 94, 178

Replacement, axiom of, 190n.2

Rigidity, definition of, 101

Rigidity, principle of, 99-102, 109-10, 114, 116n.6, 168, 191nn.6, 7

Russell, Bertrand, 1-5, 8-156, 168, 190, 193-222, 247, 279n.4

on distinction between existence and being, 121-22, 205

on events, 203-6, 217-19

on Frege, 8-10, 69, 81-82, 88-89

on matrices, $35-38,42,47,53,57$, 212-16

on numbers. See Natural numbers and classes, Russell's analysis of

on the operation and principle of substitution, 34-35, 211

on the principle of atomicity, 211, 21719

on propositional functions in his post

[PM] view, 212

on propositions in his post [PM] view, 212

on simples, 210

Russell's logical types, theory of (1908), $43-48$

Russell's "No classes" theory, 26, 28-30, 38,59 n.6, 81, 121, 156, 200

Russell's paradox, 8, 26, 69, 83-89, 102, $146,181,188,199-200,214-15$ 
and the class as one versus the class as many, 22-23, 80-81

and denotationless nominalized predicates, 103-4, 177-78

Frege on, 10, 83, 90, 102-5, 176

and Frege's logic, 64, 159, 165, 167

of membership, 23, 81, 105

of predication, 26, 59n.6, 94, 143, 145, 167,215

resolution of, 92-94, 98, 102-3, 143$47,165-68,176-77,215$

result of on logicism, $64,69,81,83$, 104-5, 152, 167

Russell's substitutional theory of classes.

See Substitutional theory

Russell's theory of denoting

early, 12, 24, 31, 122-29

1905 , or new or theory of incomplete symbols, 25-26, 29, 34, 47, 123. See also Definite descriptions

Russell's theory of truth, 1910-13, 51-52, 204

elementary truth or falsehood, second and higher order truth and falsehood in, 51-52, 198, 204

Sagen (says) versus Zeigen (shows), 23138. See also Doctrine of Showing; Zeigen

Schröder, Ernst, 89-91, 155

Second order logic, 66, 99, 113, 143-44, 146-47, 160, 194, 211, 215, 283nn.30, 34,35

modal, 135-38

of nominalized predicates, 95, 99, 105,

116n.8, 160-65, 176

ramified, 195, 202, 216, 219n.2

substitution free axiom set for, 73-74

the theory of homogeneous simple types as a, 94-97

Sellars, W., 225, 250, 279n.8

Set theory, 5-6, 66, 105, 153-56, 177, 205

general, 155

Jensen's NFU, 172-76, 180-81
Von Neumann-Bernays-Gödel, 22

Quine's ML, 10, 108, 152-53, 160, 170, 180-88

Quine's NF, 10, 59n.6, 108, 144, 152$56,160,169-72,180-81,184,186-$ 87

second order, 9, 76, 80, 99, 105, 160, 177,178

Zermelo, 10, 65, 97, 98, 116n.3, 174, 175,190 n. 2

Zermelo-Fraenkel (ZF), 154, 156

Simple types, theory of, 89-94

homogeneous, 94-97, 167

as a theory of classes, 172

Situations (Sachlage)

atomic, 234, 241, 243n.13, 246, 24953, 258-61, 267, 271, 275-77, $278 \mathrm{n} .1,279 \mathrm{n} .8,281 \mathrm{nn} .18,21$, 283 n. 32

atomic and complementation, 224-26. See also Complementation between atomic situations

complex, 234, 281n.18

Specker, Ernst, 156, 172, 174

Spiritus Asper (rough breathing abstraction operator), 82-83

Spiritus lenis (smooth breathing abstraction operator), 9, 82-83, 160

States of affairs, 30, 224, 226, 231, 234.

See also Situations, atomic

State descriptions, Carnap's, 265, 282n.29

Statements versus Propositions, 38-42, 60n.13, 61nn.16, 17, 204

Subsistence (Bestehen), 126-27, 200, 205, 243n.12. See also Being

Substitution free axiom set for second order predicate logic, 73-74

Substitution rule, 233-35, 243n.12, 256, 266-67, 276, 279n.9, 280n. 10

Substitutional theory of classes, 29, 33-46, 59 n.4. See also Russell's "No classes" theory

Syncategorematic signs, 254-56, 272, 279n.7

Syncategorematic tie, 224, 243n.12, 24854,279 n. 5 
Tarski, A., 54, 76, 216, 264-65

Tractarian or model-theoretic ladder, 225 $26,245,256,259-60,272,278 \mathrm{n} .2$

Transformational grammar, 85

Truth. See Logical truth; Russell's theory of truth

Truth, Tarski's notion of, 264

Type theory. See Logical types, theory of

Ultimate dualism, Russell's, 205

Ultimate (proper) classes, 181-88

Universals, 16, 49, 104, 200-6, 208-9, $211,251-52$

Universals and particulars, Russell's ultimate dualism of, 203-6

Unrestricted satisfaction principle, 125 , 127,140

Unsaturatedness (or unsaturated nature), $10,67-68,72,81-82,91,103,111-$ $12,160,178,251,280 \mathrm{n} .12$

Urmson, J. O., 17nn.1, 2

Urelements, 153-56, 169, 172-76, 185

Validity, universal, 233, 238

Validity with respect to a cardinal number $k \leqslant \chi_{0}, 273,275,282$ n.29
Value-ranges (Wertverläufe), 76-79, 80, $83,99,102,158,160$ as concept-correlates, 76-80, 102, 158

Vicious-circle principle, the, 38-39, 41, 43

Wang, Hao, 108, 191n.12

"Watering down" axiom, 139-40

Wertverläufe (value-ranges), 76-79, 158. See also Value-ranges

Wittgenstein, Ludwig, 12-16, 57, 196, 207-8, 217, 222, 250, 279n.4

The World (actual or real) in logical atomism, 226, 232, 252-53, 270

World propositions, 15. See also Possible worlds as world facts

Zermelo set theory. See Set theory, Zermelo

Zeigen (show), 229, 231, 236, 245, 255, 274, 276, 282n.25, 283n.32. See also Doctrine of Showing versus Sagen (says), 231-38, 255, 272, 278n.2, 280n. 17

Zig-zag theory, 28, 59n.6 


\section{A NOTE ABOUT THE AUTHOR \\ Nino B. Cocchiarella is Professor of Philosophy at Indiana University. $\mathrm{He}$ is the author of Logical Investigations of Predication Theory and the Problem of Universals. \\ This book was set in Sabon by Huron Valley Graphics, Ann Arbor, Michigan. \\ Text design by Mary Mendell.}

